\title{
THE PASSAGE TOWARD STALL OF NONSLENDER DELTA WINGS AT LOW REYNOLDS NUMBER
}

\author{
Thesis by \\ Michael V. OL \\ In Partial Fulfillment of the Requirements for the Degree of \\ Doctor of Philosophy \\ California Institute of Technology \\ Pasadena, California
}

2001

(Submitted December 1, 2000) 
(C) 2001

Michael V. OL All Rights Reserved. 


\section{Acknowledgments}

As we plod along "The Course of Science," I wish to thank first those who were most responsible for motivating the perhaps Quixotic but richly rewarding "doctrine" of Olism: my comrades in the Aeronautics department, the present and future Drs., in alphabetical order, Brad Dooley, Dave Jeon, Paul Krueger, Piet Moeleker, Flavio Noca, Han Park, Jerry Shan, Heinrich Stueer, and numerous others. They provided invaluable guidance, assistance, and commiseration in my various endeavors. Han Park and Dave Jeon deserve special thanks for extracting me from the quagmire of software development.

Messrs. Rich Eastvedt, Russ Green and Mike Vondrus, of the Keck Hydraulics $\mathrm{Lab}$, were invaluable in assisting with the construction of my laboratory, as were numerous other gentlemen on campus who lack the title "Dr.," but whose hands-on engineering insight is no less impressive.

I wish to thank my present and former colleagues and mentors at the "Air Vehicles" directorate of the AFRL; in particular, Jerry Jenkins, Chuck Jobe, and Dieter Multhopp. Special thanks also to Carl Tilmann, for preventing me from tearing my hair out during the task of typing this thesis. My career as a government employee has been profoundly instructive in shaping both the present work, and my capacity to perform it.

Among the Cal Tech faculty, I am indebted to Prof. Brooks, for his clever suggestion on how to solve the "sloshing problem" of my water tunnel; and of course to my committee, Profs. Gharib, Hornung, Leonard, and Pullin, not only for their academic guidance, but far more so for their generous assessment that I actually deserve a Ph.D. And of course, I am indebted to my advisor, Prof. Mory Gharib, from whom I learned much about fluid mechanics and of the flow of the world at large.

It is of course implicit that I also thank that which authors usually thank. For barring that, this thesis would have been utterly impossible. The most profound thanks are generally left unsaid. 
This work is dedicated to Arina Ostrakhovich, whose rather long name will hopefully soon be much shorter. Only with her love and patience has it been worth trying to survive. 


\section{Preamble}

At present, the delta wing field, like most areas of aerodynamics, is often described by the more than vaguely pejorative term "mature." Purportedly, the substantive problems have been already solved or else declared unsolvable, leaving present researchers fighting for the few piddling edible crumbs that remain. But amidst this unhappy conclusion there is the ever-present development of adjunct disciplines and new technologies, making possible new approaches to old problems in "classical" fields and thereby, new material for publication. One example is that of new diagnostic techniques, which allow for further refinement of measurements, in this case of the velocity field. New results are stumbled upon by the application of such novel techniques, rather than by exceptional conceptual insight or inventive drive.

bread.

So, while most of us must make do with gathering crumbs, these crumbs are our 


\section{Abstract}

Separated flow over the leeside of relatively nonslender delta wings was studied experimentally. Such flowfields are more complex than those of the slender delta wing of very low aspect ratio. A version of Stereo Digital Particle Image Velocimetry was applied to measurements in a low speed water tunnel, at Reynolds numbers below 20,000 , for delta wing models of $50^{\circ}$ and $65^{\circ}$ leading edge sweep angles and $30^{\circ}$ windward-side leading edge bevels. Since the objective was to draw comparisons to the stall of classical high aspect ratio wings, low angles of attack were emphasized, with most data points taken in the $5^{\circ}-20^{\circ}$ angle of attack range. Measurements were taken over the starboard portion of the wing planform in crossflow planar slices near the apex region, yielding all three components of the velocity field, albeit restricted to planar cuts. Vorticity and circulation were calculated from these measurements. All three components of vorticity were obtained in select cases, by central-differencing velocity data across triplets of adjacent interrogation planes. In addition, flow visualization by dye injection into the windward apex stagnation region was used to confirm the presence of primary and secondary leading edge vortices, to qualitatively verify the locations of vortex breakdown, and to verify the stereo digital particle image velocimetry results.

Both delta wings exhibit stable, coherent leading edge vortices at very low angles of attack, down to $2.5^{\circ}$. Results for the $65^{\circ}$ wing were in accordance with the literature. The $50^{\circ}$ wing, however, exhibited flow characteristics akin to both slender delta wings, and wings of high aspect ratio, and generally exhibited stronger and more robust leading edge vortices than usually observed. For the $50^{\circ}$ wing, the primary leading edge vortices were stable below $10^{\circ}$ angle of attack, with gradual and steady upstream progression of 
the vortex breakdown region with increasing angles of attack, from aft of the trailing edge to approximately the midchord. Secondary leading edge vortices were found to decay more abruptly, and at lower angle of attack than the primaries, all but disappearing by $10^{\circ}$ angle of attack. This fact has the potential of serving as the basis for a predictive criterion for breakdown of the primary vortices.

The entire vortex system undergoes large-scale instabilities in the $12^{\circ}-20^{\circ}$ angle of attack range. While the flow visualization was inconclusive, particle image velocimetry confirmed that breakdown sweeps over the entire forward third of the wing planform in going from $12.5^{\circ}$ to $15^{\circ}$ angle of attack. This change is characterized by a sharp drop in axial velocity in the primary leading edge vortex core region, along with a loss of coherent vortical structure normally associated with this region. The leading edge shear layer, however, remains in an organized rolled-up state. By $20^{\circ}$, the flow over the leeward side of the wing is at the threshold of complete separation, with flow along the wing centerline stalling as the left and right separated regions grow and merge.

Both wings exhibited a largely stagnant region outboard of the primary LEV and inboard of the leading edge shear layer, especially at angles of attack beyond $10^{\circ}$. This phenomenon is consistent with some prior observations at Reynolds numbers on the order of 20,000 and below, and differs sharply from that at higher Reynolds numbers. Further experiments are necessary to elucidate the cause and extent of Reynolds number influence on separation near the leeward surface. Also, the $50^{\circ}$ wing is probably of too high sweep to be a true limiting case for the existence of coherent leading edge vortices, for the conditions of the present experiment. But the abruptness of its stall and the close relationship between the leading edge vortex flow and the leeward surface boundary layer 
are qualitatively indicative of such a transitional case from slender delta wing separation to classical airfoil stall. 


\section{Table of Contents}

Acknowledgments. .iii

Preamble. v

Abstract vi

Table of Contents ..ix

List of Figures xiii

List of Tables. $\mathrm{xviii}$

List of Symbols xix

Chapter 1 Introduction 1

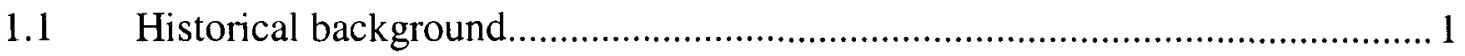

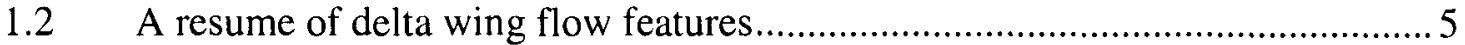

1.2.1 Leading edge separation and shear layer rollup ......................................... 6

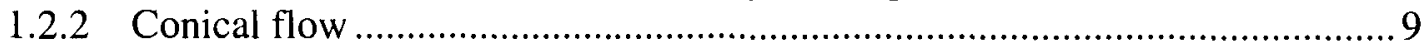

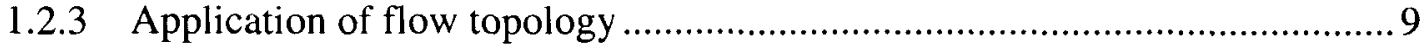

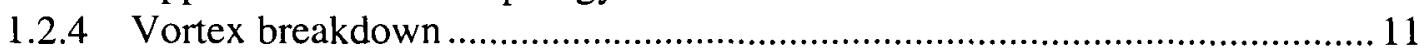

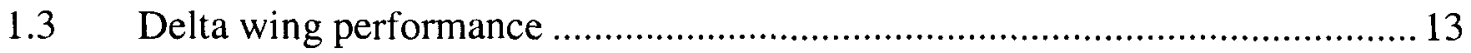

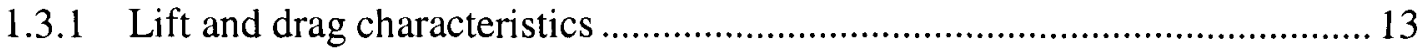

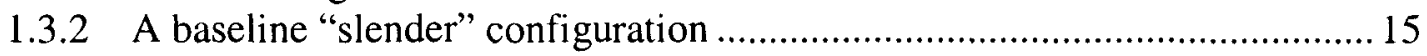

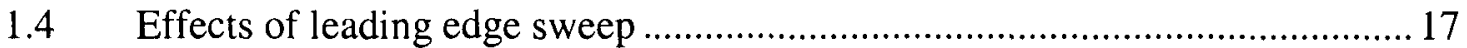

1.5 Implications of delta wing theoretical models................................................20

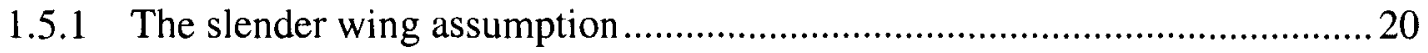

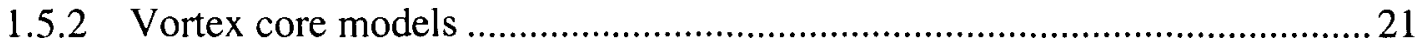

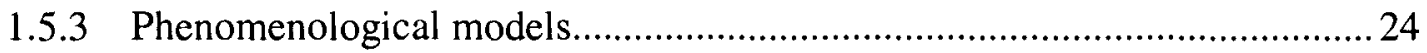

1.6 Leading edge vortex stability vs. existence ................................................... 25

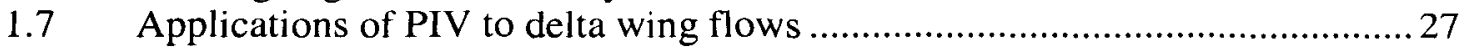

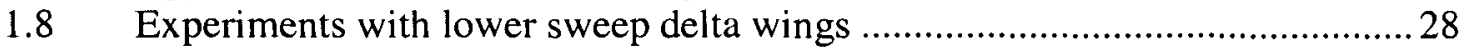

1.9 Prospects and conjectures ............................................................................ 31

Chapter 2 Rotational Stereo Digital PIV in a Water Tunnel........................................... 33

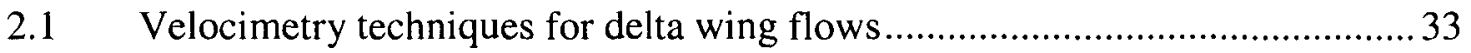

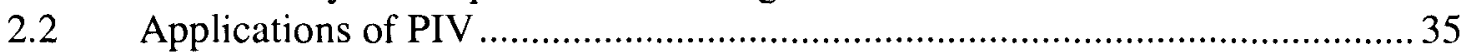

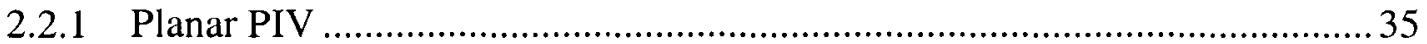

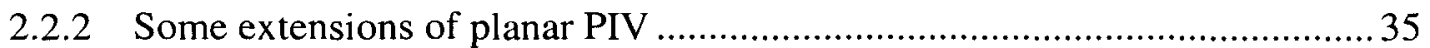

2.3 Stereo PIV applied to the present experiment ................................................ 37 


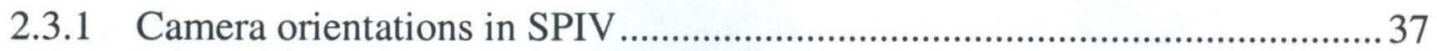

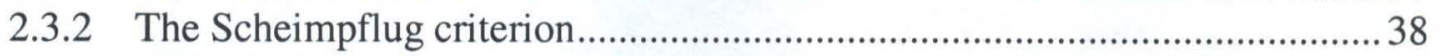

2.3.3 Rotational camera arrangement.............................................................. 40

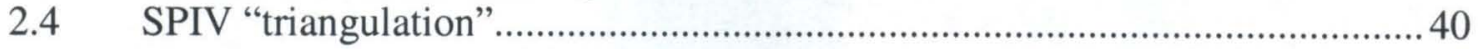

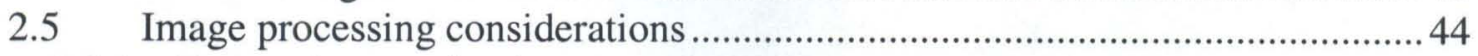

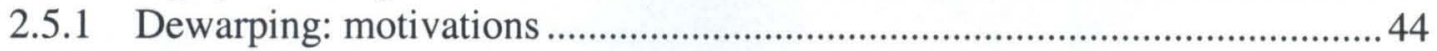

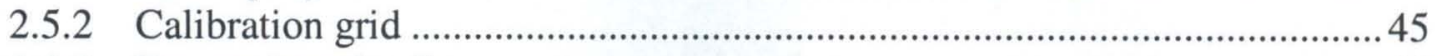

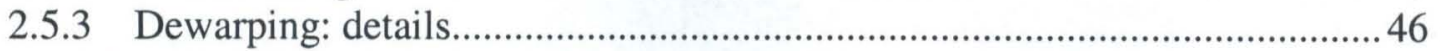

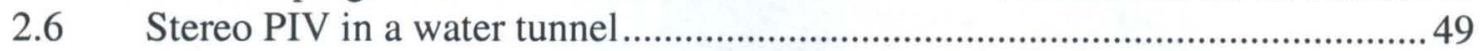

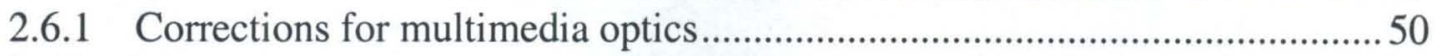

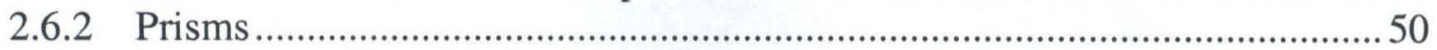

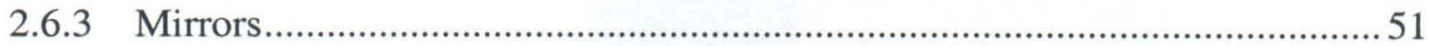

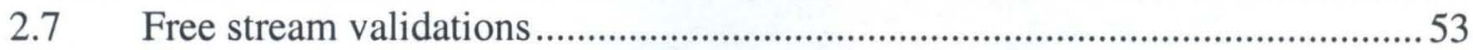

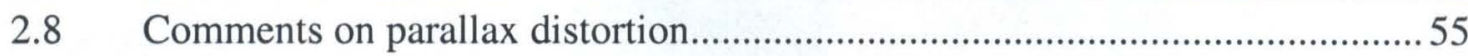

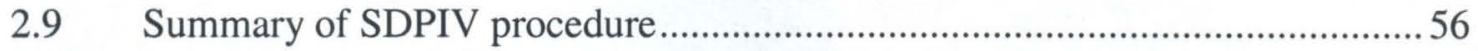

2.10 Application to a full-span measurement of the delta wing flow field ...............57

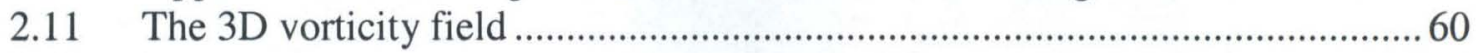

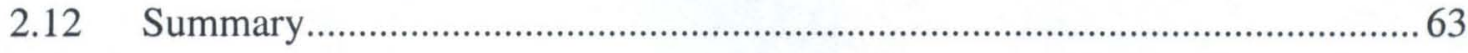

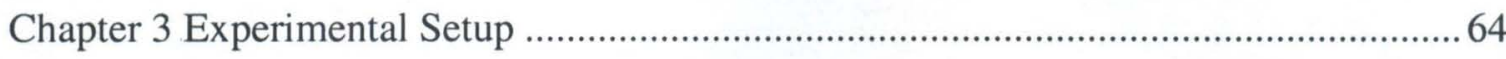

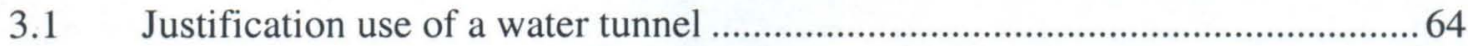

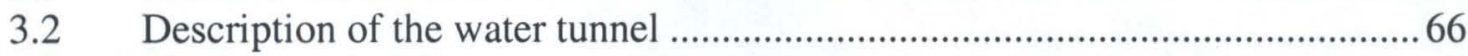

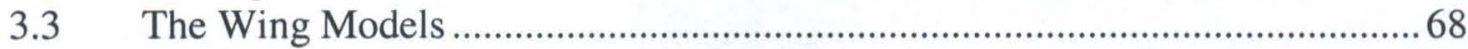

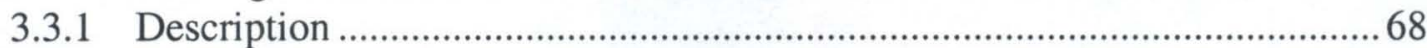

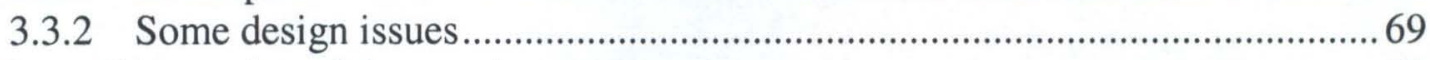

3.4 Sting and model mounting system............................................................... 71

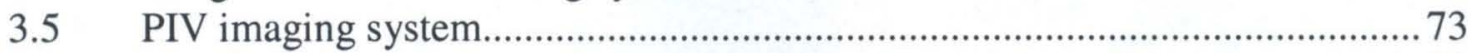

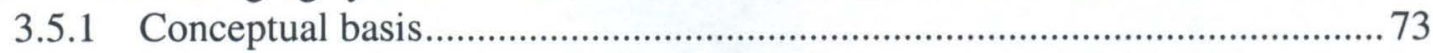

3.5.2 Laser light sheet optics.............................................................................. 74

3.5.3 Laser light sheet geometry issues................................................................76

3.5.4 Particles in the LEV core ……………..................................................... 79

3.5.5 Cameras and camera triggering ................................................................ 80

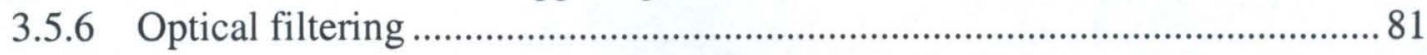

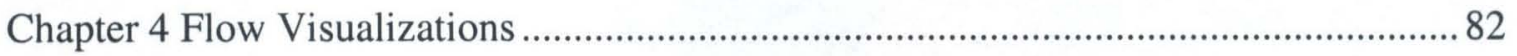

4.1 General description of flow visualization issues ............................................ 82

4.1.1 Core trajectory and vortex breakdown ....................................................... 83

4.1.2 Dye injection issues: straight vs. wavy trajectories......................................... 84

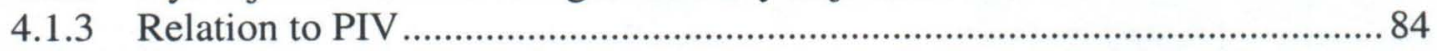

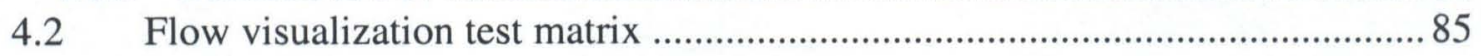

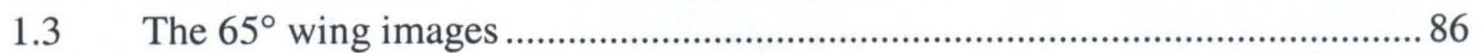

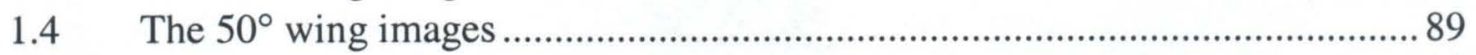

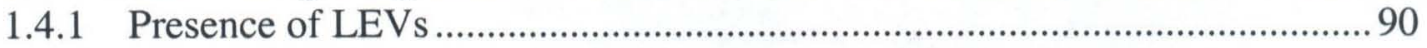

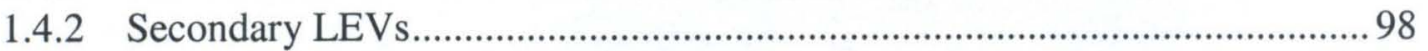

1.4.3 Evidence of unsteadiness upstream of breakdown....................................... 101 


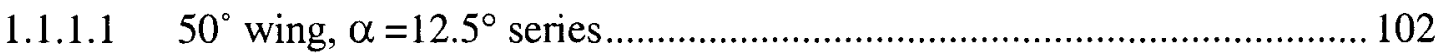

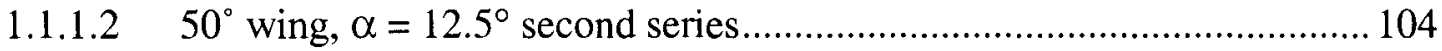

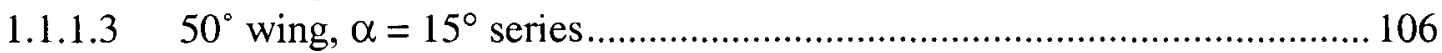

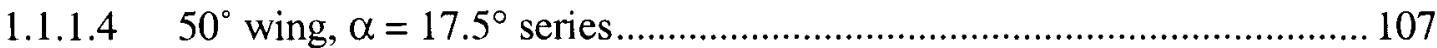

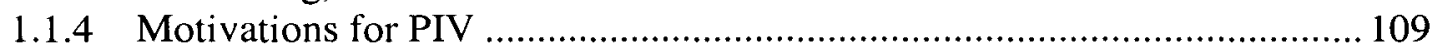

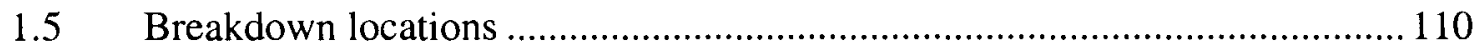

1.6 Core axial speed measurements............................................................ 113

1.7 Summary, and comments on the distinction between the $65^{\circ}$ and $50^{\circ}$ wings 114

Chapter 5 SDPIV Data in Crossflow Planes ................................................................. 117

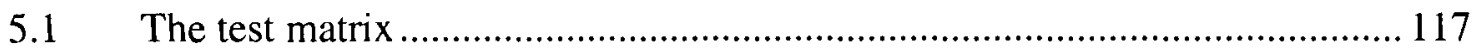

5.1.1 Schedule of angle of attack sweeps....................................................... 117

5.1.2 Schedule of streamwise station sweeps...................................................118

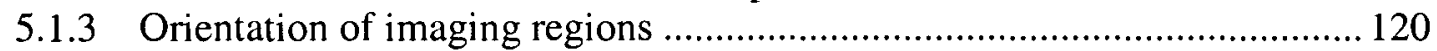

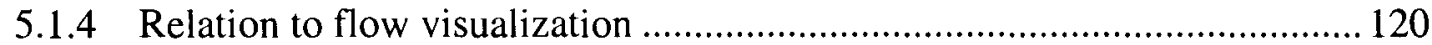

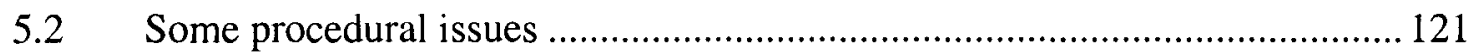

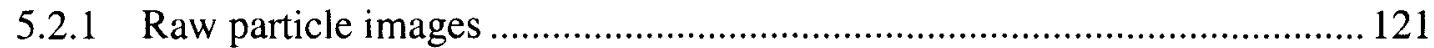

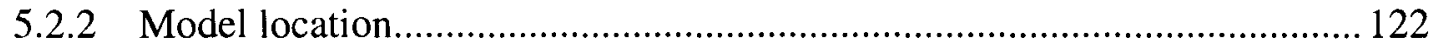

$5.3 \quad$ Resume of velocity vector field plots ...................................................... 123

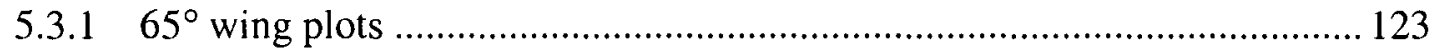

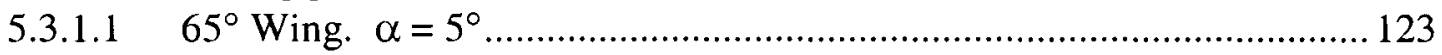

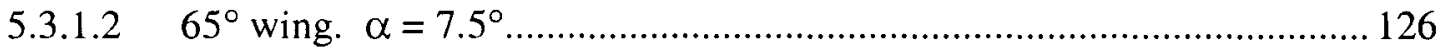

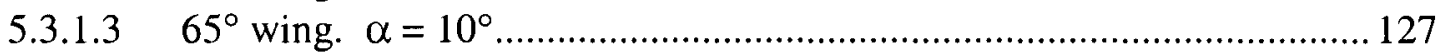

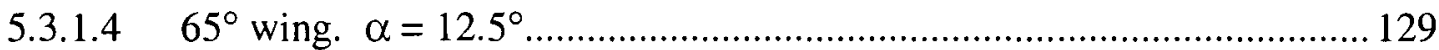

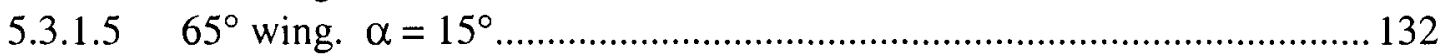

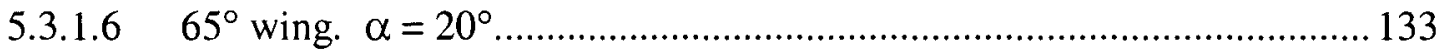

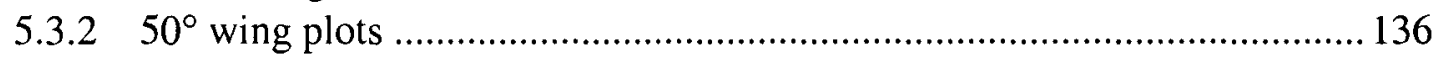

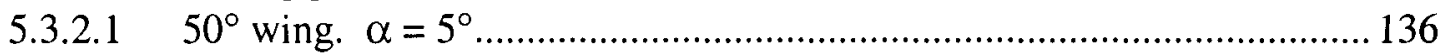

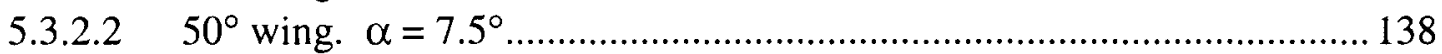

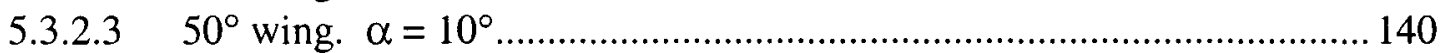

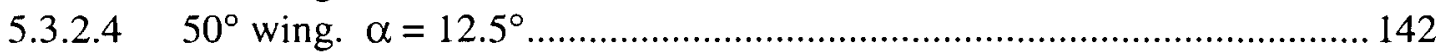

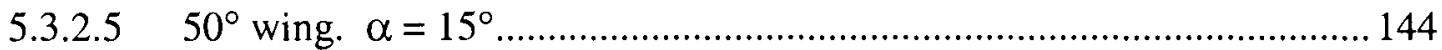

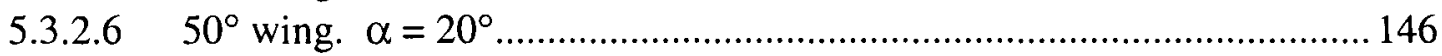

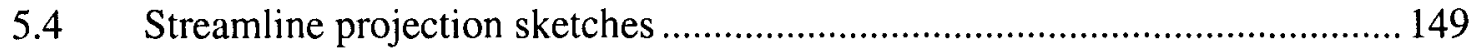

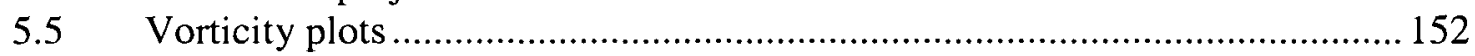

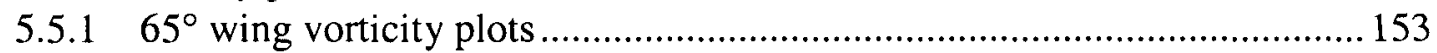

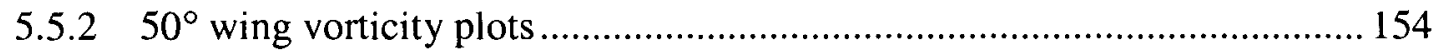

5.5.3 3-components of vorticity for selected cases ............................................ 156

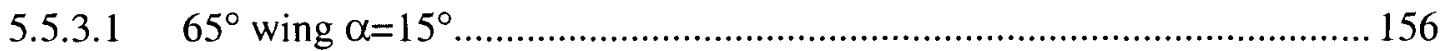

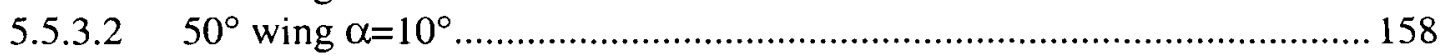

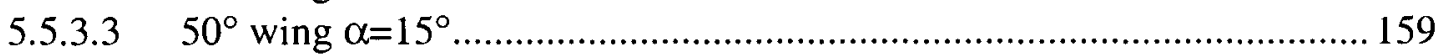

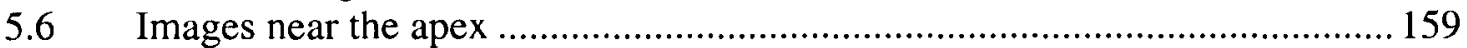

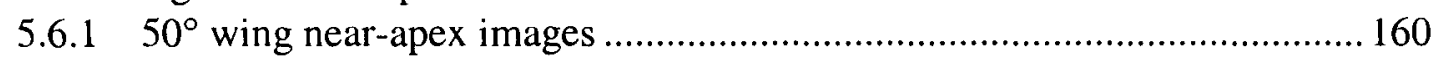

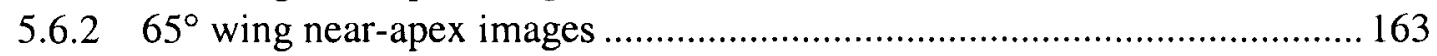


5.6.3 A summary of near-apex flow features ....................................................... 165

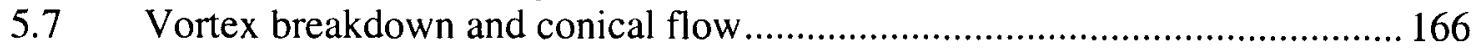

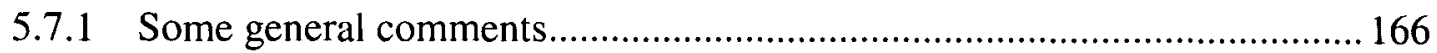

5.7.2 The $50^{\circ}$ wing at intermediate angles of attack .......................................... 167

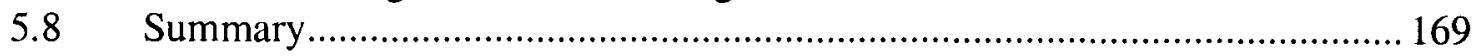

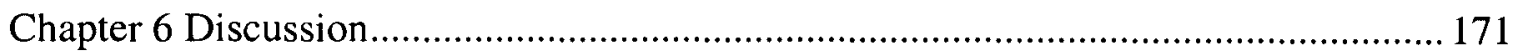

6.1 Comparison of flow visualization and SPIV results................................ 171

6.2 Crossflow plane velocity profiles .................................................................. 174

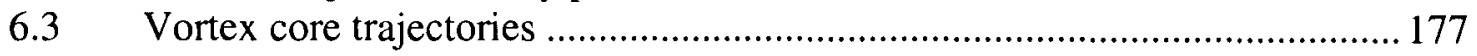

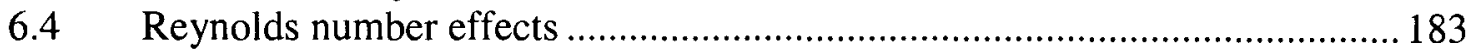

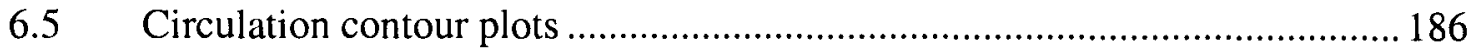

6.6 Comparison with an LEV stability model .............................................. 190

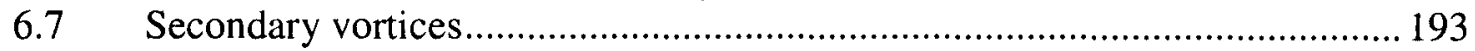

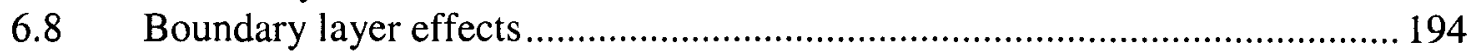

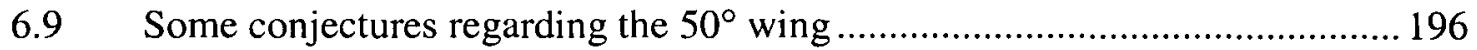

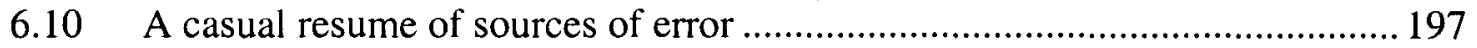

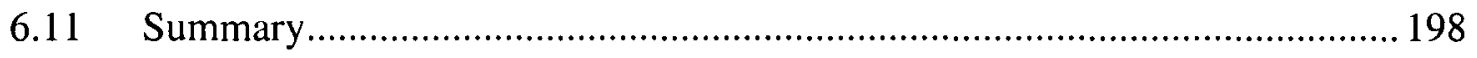

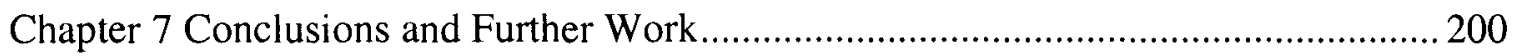

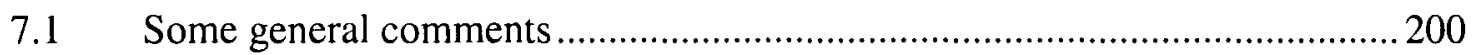

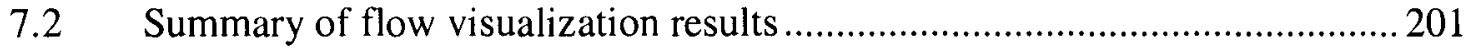

7.3 Summary of stereoscopic particle image velocimetry results ........................ 202

7.4 The passage toward stall for the $50^{\circ}$ delta wing at low Reynolds number..... 204

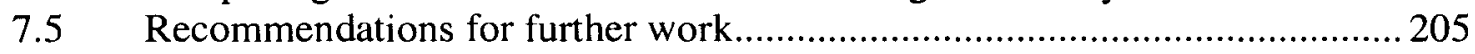

Appendix AThe Water Tunnel and Sloshing........................................................... 210

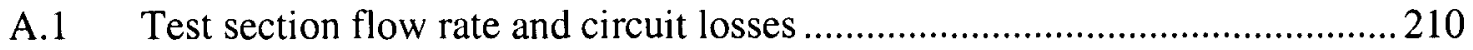

A.2 Some comments on turbulence intensity ...................................................21

A.3 Sloshing: time traces of test section flow velocity ....................................... 212

Appendix B SPIV Validations and Correction of Parallax Distortion ............................ 216

B.1 Orthogonal translations of an artificial particle grid .................................... 216

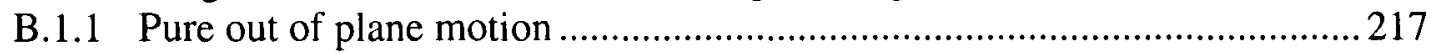

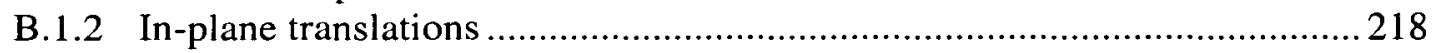

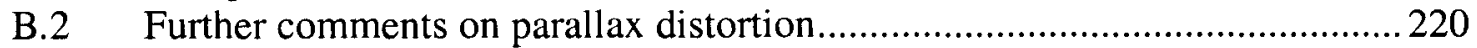

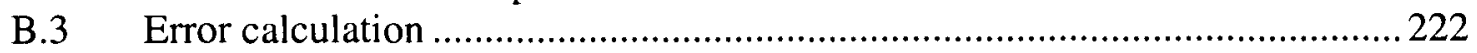

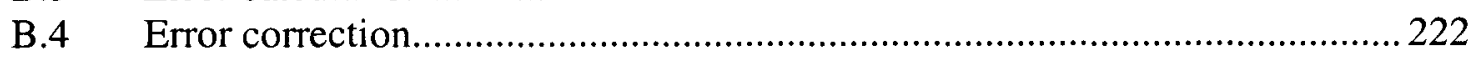

Appendix CMitror-Related SPIV Geometric Calculations .............................................2225

C.1 Triangulation parameters for standard camera arrangement .........................2225

C.2 Triangulation parameters for arrangement with mirrors ................................222

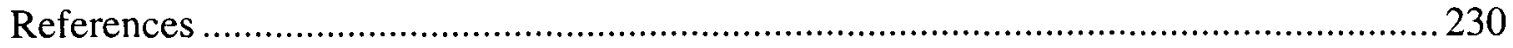




\section{List of Figures}

Figure 1.1. Schematic of the delta wing flow field (based on Hoerner) ............................................... 6

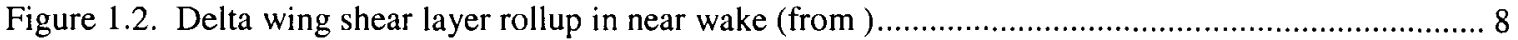

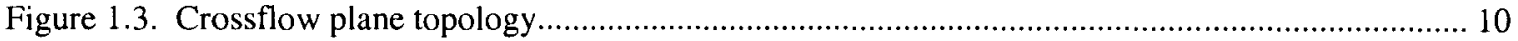

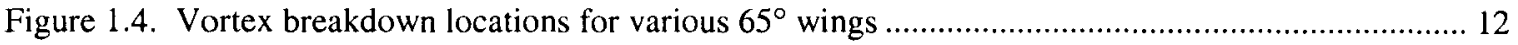

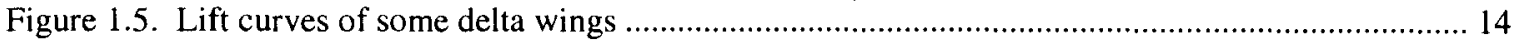

Figure 1.6. Drag polar for delta wings of 45 to 76 degree sweep ........................................................ 14

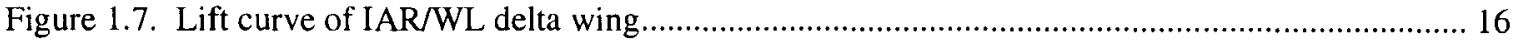

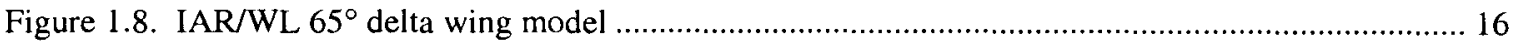

Figure 1.9. Nine types of flowfields of delta wings (from Su et al.) ................................................ 17

Figure 1.10. Effect of LE sweep angle of delta wing flow features...................................................... 19

Figure 1.11. Theoretical and experimental axial velocity profiles ...................................................... 22

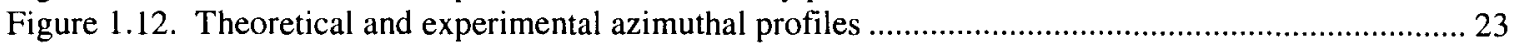

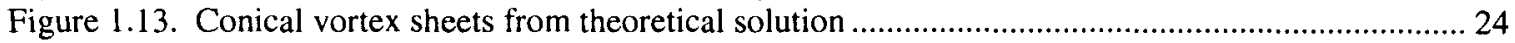

Figure 1.14. LEV stability diagram (from Wedemeyer) .............................................................. 26

Figure 2.1. Photograph of stereo PIV camera and lens arrangement .................................................... 39

Figure 2.2. Camera and lens arrangements with Scheimpflug criterion, top view ................................. 40

Figure 2.3. Stereoscopic representation of displacement vector......................................................... 41

Figure 2.4. Stereoscopic representation of displacement vector, top view ......................................... 42

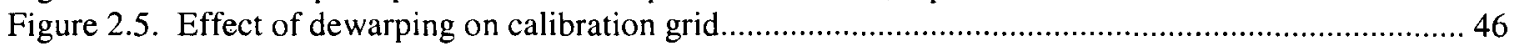

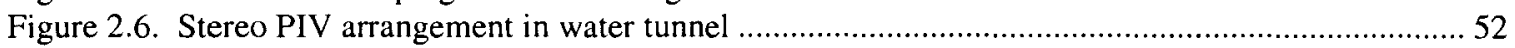

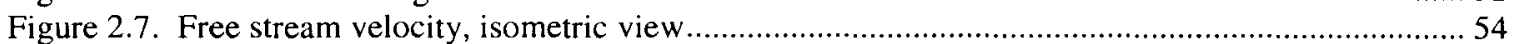

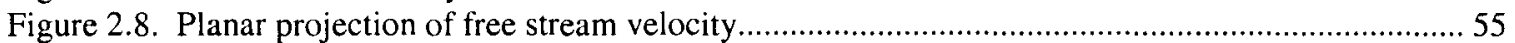

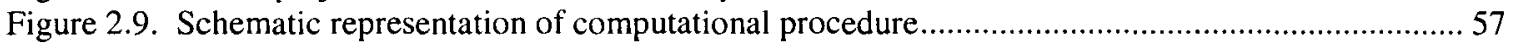

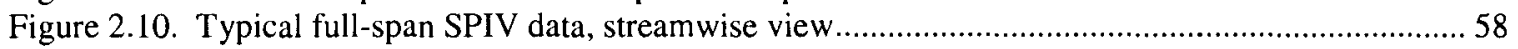

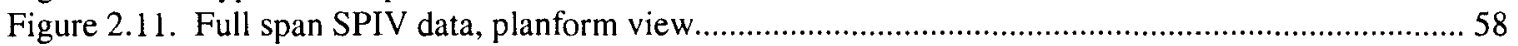

Figure 2.12. Normal velocity component along line passing through LEV cores ..................................59

Figure 2.13. Azimuthal velocity along line passing through LEV cores .............................................. 59

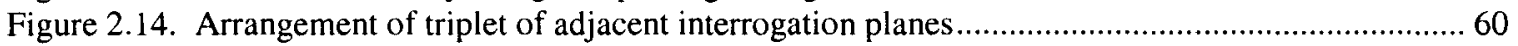

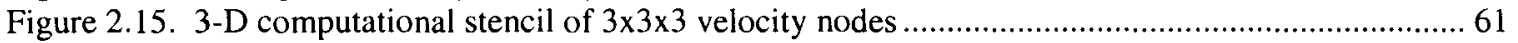

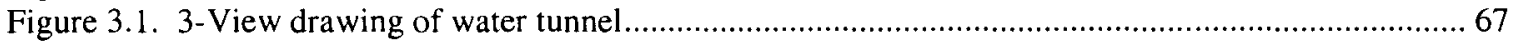

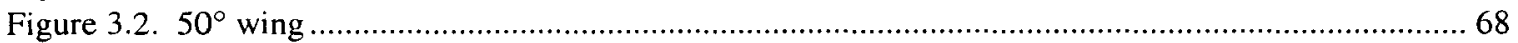

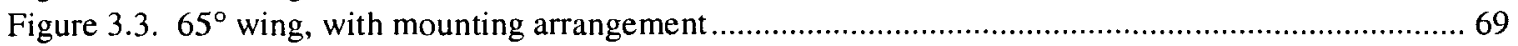

Figure 3.4. 3-View drawing of test section, model mounting and optics ................................................ 73

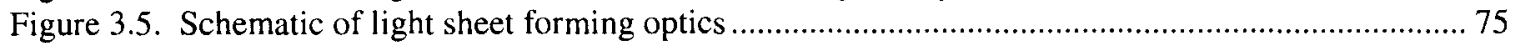

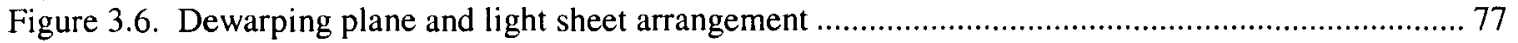

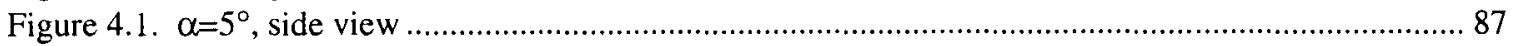

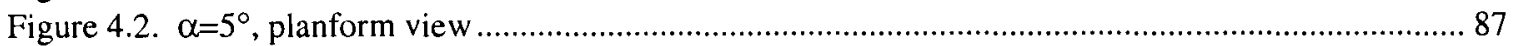

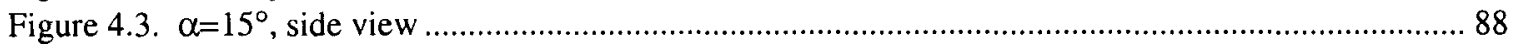

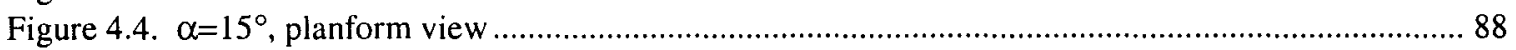

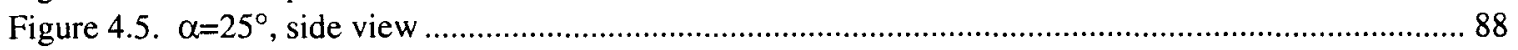

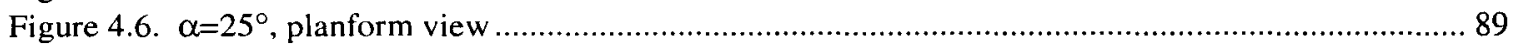

Figure 4.7. $\alpha=2.5^{\circ}$, side view ................................................................................................. 91

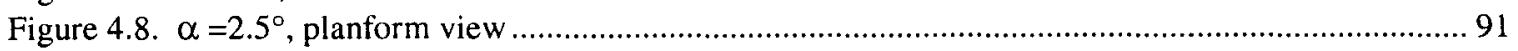

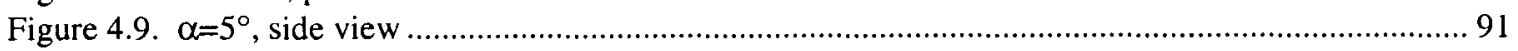

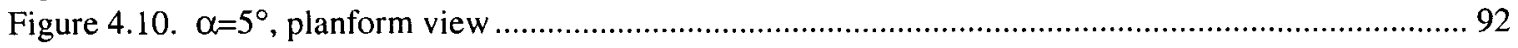

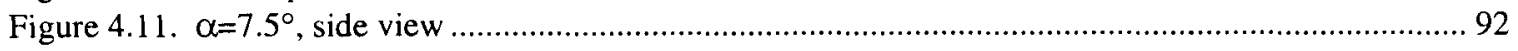

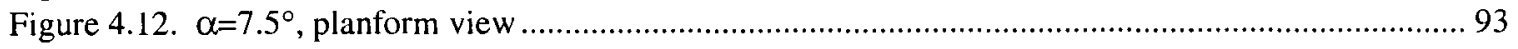

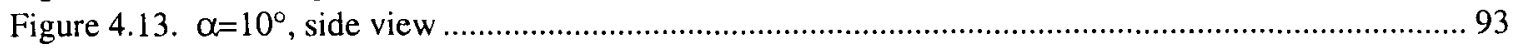

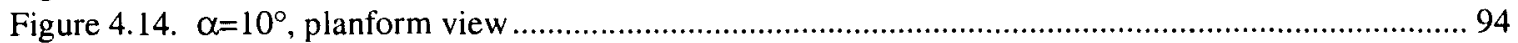

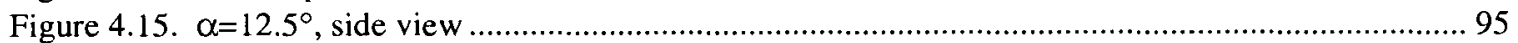

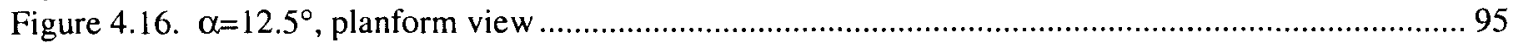

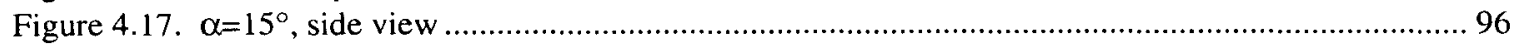




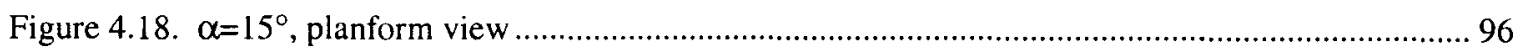

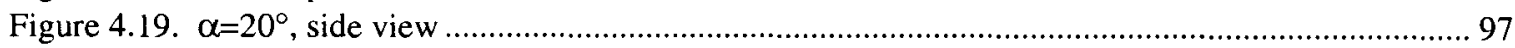

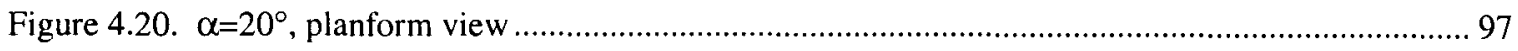

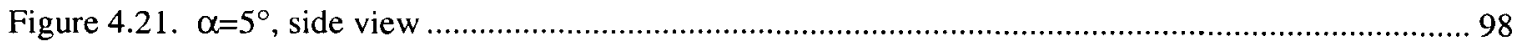

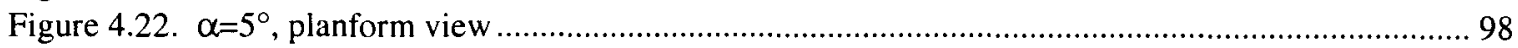

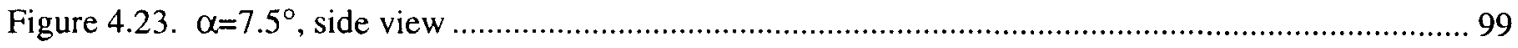

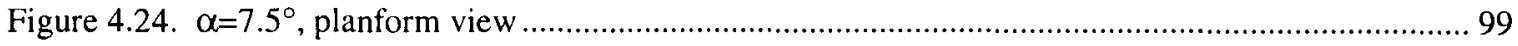

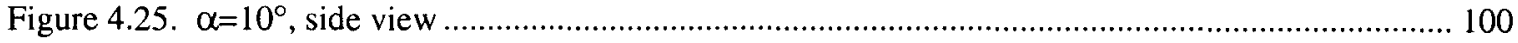

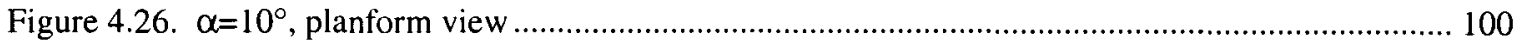

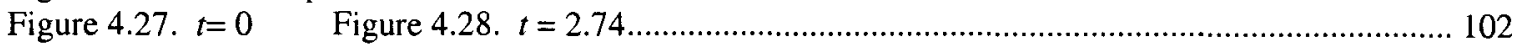

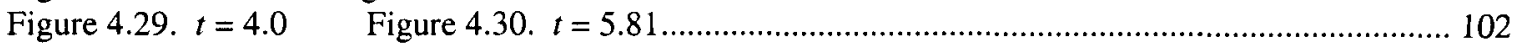

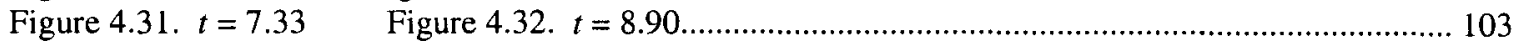

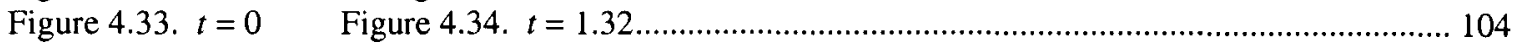

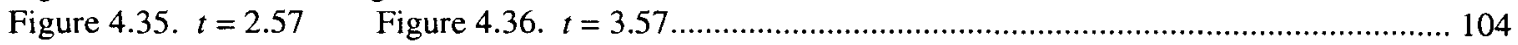

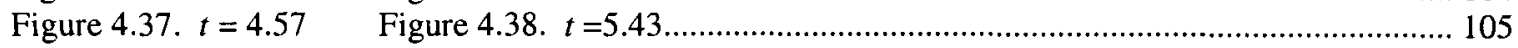

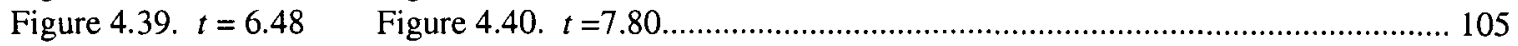

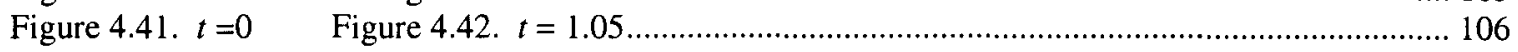

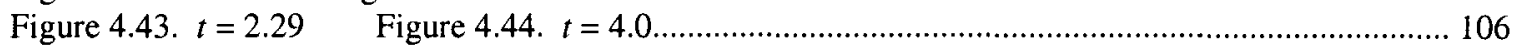

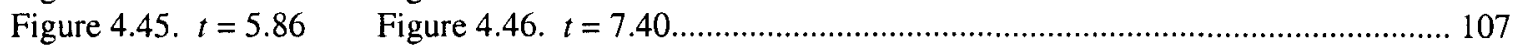

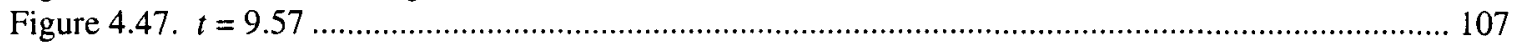

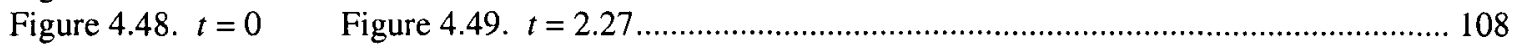

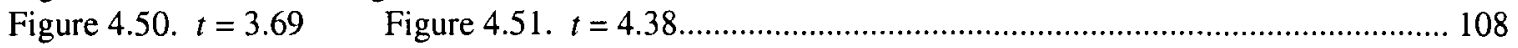

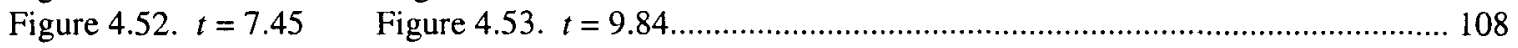

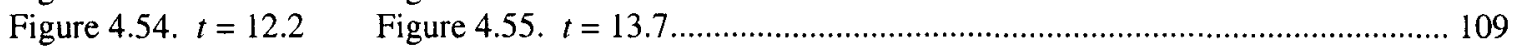

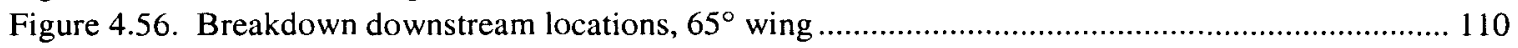

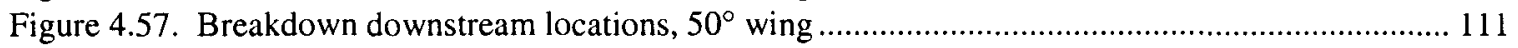

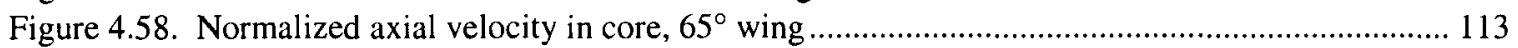

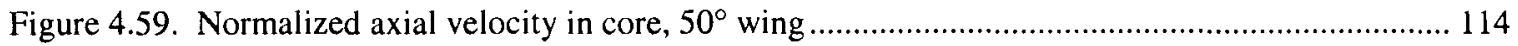

Figure 5.1. Arrangement of stereo PIV interrogation planes............................................................. 120

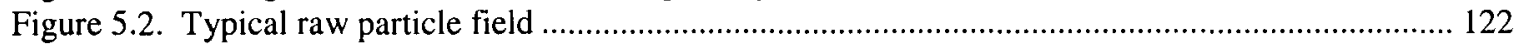

Figure 5.3. $65^{\circ}$ wing, $\alpha=5^{\circ}$; typical left and right camera views (dewarped)................................... 122

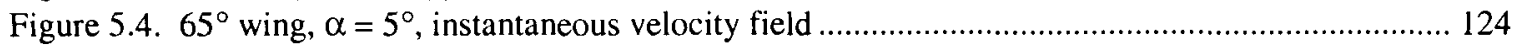

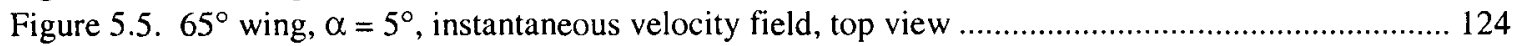

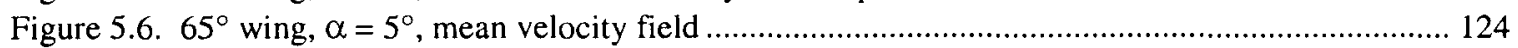

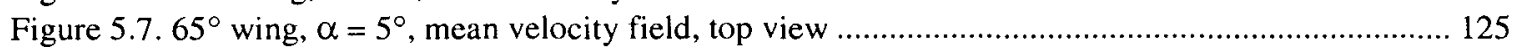

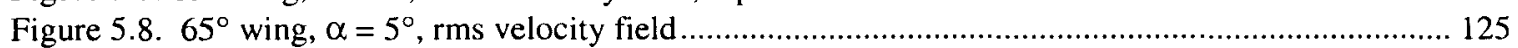

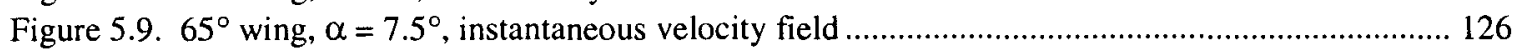

Figure 5.10. $65^{\circ}$ wing, $\alpha=7.5^{\circ}$, instantaneous velocity field, top view ........................................... 126

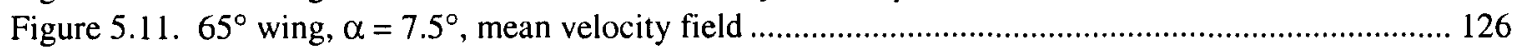

Figure 5.12. $65^{\circ}$ wing, $\alpha=7.5^{\circ}$, mean velocity field, top view........................................................ 127

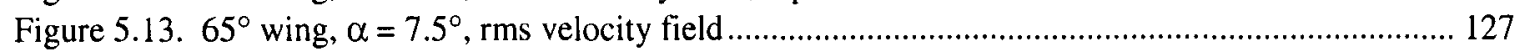

Figure 5.14. $65^{\circ}$ wing, $\alpha=10^{\circ}$, instantaneous velocity field ........................................................ 128

Figure 5.15. $65^{\circ}$ wing, $\alpha=10^{\circ}$, instantaneous velocity field, top view ............................................. 128

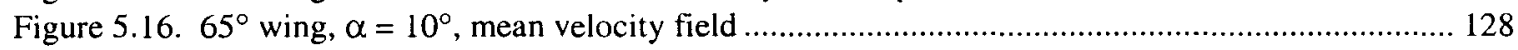

Figure 5.17. $65^{\circ}$ wing, $\alpha=10^{\circ}$, mean velocity field, top view..................................................... 129

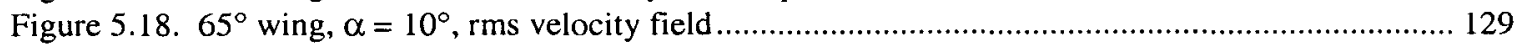

Figure 5.19. $65^{\circ}$ wing, $\alpha=12.5^{\circ}$, instantaneous velocity field .................................................... 130

Figure 5.20. $65^{\circ}$ wing, $\alpha=12.5^{\circ}$, instantaneous velocity field, top view ........................................... 130

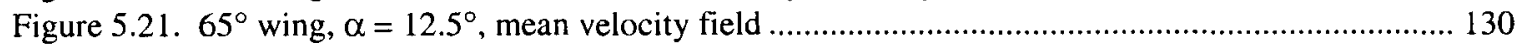

Figure 5.22. $65^{\circ}$ wing, $\alpha=12.5^{\circ}$, mean velocity field, top view.................................................. 131

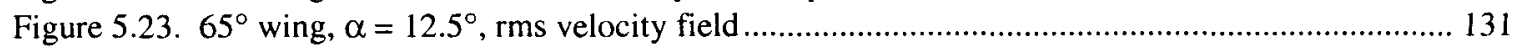

Figure 5.24. $65^{\circ}$ wing, $\alpha=15^{\circ}$, instantaneous velocity field ..................................................... 132

Figure 5.25. $65^{\circ}$ wing, $\alpha=15^{\circ}$, instantaneous velocity field, top view ............................................ 132 


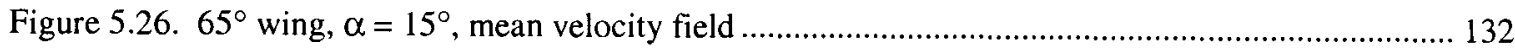

Figure 5.27. $65^{\circ}$ wing, $\alpha=15^{\circ}$, mean velocity field, top view..................................................... 133

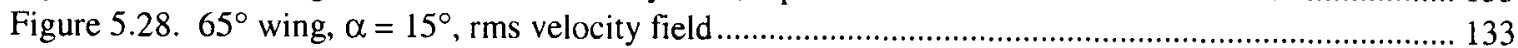

Figure 5.29. $65^{\circ}$ wing, $\alpha=20^{\circ}$, instantaneous velocity field ....................................................... 134

Figure 5.30. $65^{\circ}$ wing, $\alpha=20^{\circ}$, instantaneous velocity field, top view ............................................. 134

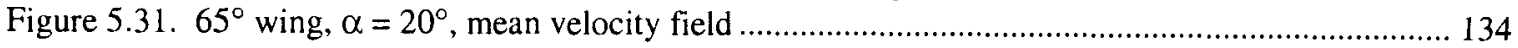

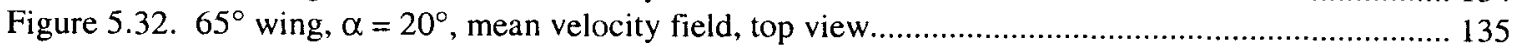

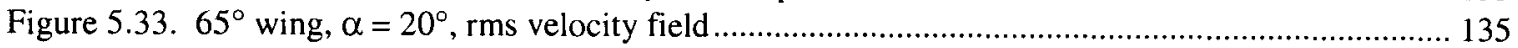

Figure 5.34. $50^{\circ}$ wing, $\alpha=5^{\circ}$, instantaneous velocity field ........................................................ 136

Figure 5.35. $50^{\circ}$ wing, $\alpha=5^{\circ}$, instantaneous velocity field, top view ............................................... 136

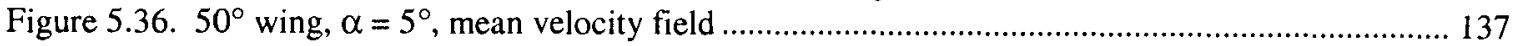

Figure 5.37. 50 wing, $\alpha=5^{\circ}$, mean velocity field, top view .......................................................... 137

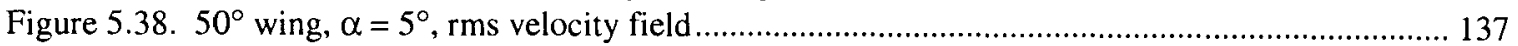

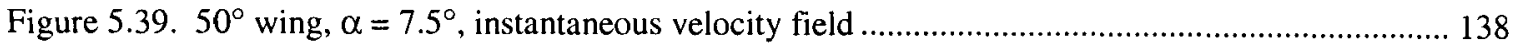

Figure 5.40. $50^{\circ}$ wing, $\alpha=7.5^{\circ}$, instantaneous velocity field, top view ......................................... 138

Figure 5.41. $50^{\circ}$ wing, $\alpha=7.5^{\circ}$, mean velocity field ................................................................... 139

Figure 5.42. $50^{\circ}$ wing, $\alpha=7.5^{\circ}$, mean velocity field, top view........................................................ 139

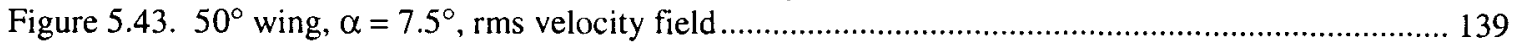

Figure 5.44. $50^{\circ}$ wing, $\alpha=10^{\circ}$, instantaneous velocity field ...................................................... 140

Figure 5.45. $50^{\circ}$ wing, $\alpha=10^{\circ}$, instantaneous velocity field, top view ........................................... 140

Figure 5.46. $50^{\circ}$ wing, $\alpha=10^{\circ}$, mean velocity field ...................................................................... 141

Figure 5.47. $50^{\circ}$ wing, $\alpha=10^{\circ}$, mean velocity field, top view.................................................... 141

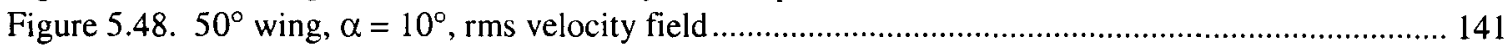

Figure 5.49. $50^{\circ}$ wing, $\alpha=12.5^{\circ}$, instantaneous velocity field ........................................................ 142

Figure 5.50. $50^{\circ}$ wing, $\alpha=12.5^{\circ}$, instantaneous velocity field, top view ........................................... 142

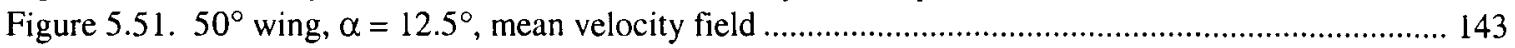

Figure 5.52. $50^{\circ}$ wing, $\alpha=12.5^{\circ}$, mean velocity field, top view.................................................. 143

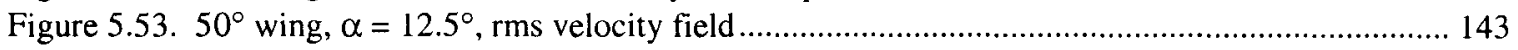

Figure 5.54. $50^{\circ}$ wing, $\alpha=15^{\circ}$, instantaneous velocity field .................................................... 144

Figure 5.55. $50^{\circ}$ wing, $\alpha=15^{\circ}$, instantaneous velocity field, top view ........................................... 145

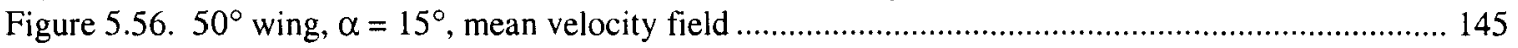

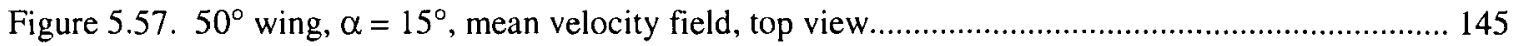

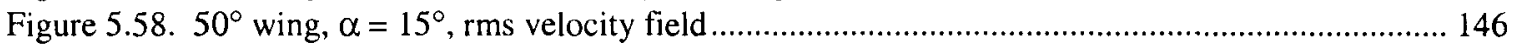

Figure 5.59. $50^{\circ}$ wing, $\alpha=20^{\circ}$, instantaneous velocity field .......................................................... 146

Figure 5.60. $50^{\circ}$ wing, $\alpha=20^{\circ}$, instantaneous velocity field, top view ............................................. 147

Figure 5.61. $50^{\circ}$ wing, $\alpha=20^{\circ}$, instantaneous velocity field, another sample .................................... 147

Figure 5.62. $50^{\circ}$ wing, $\alpha=20^{\circ}$, mean velocity field ................................................................... 147

Figure 5.63. $50^{\circ}$ wing, $\alpha=20^{\circ}$, mean velocity field, top view................................................... 148

Figure 5.64. $50^{\circ}$ wing, $\alpha=20^{\circ}$, rms velocity field ........................................................................ 148

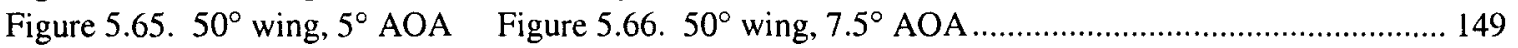

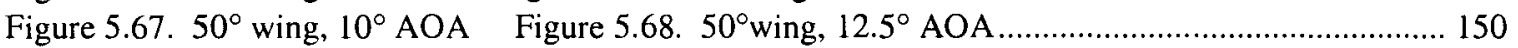

Figure 5.69. $50^{\circ}$ wing, $15^{\circ}$ AOA Figure 5.70. 50 wing, $20^{\circ}$ AOA …........................................... 150

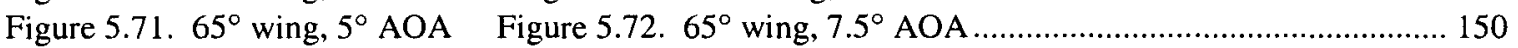

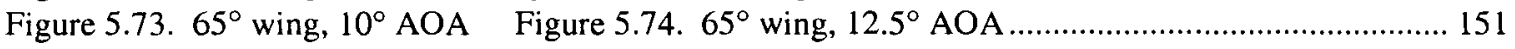

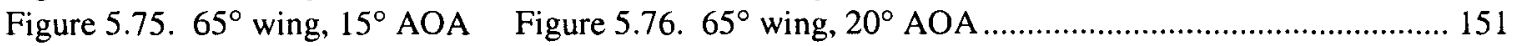

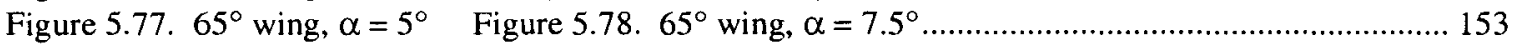

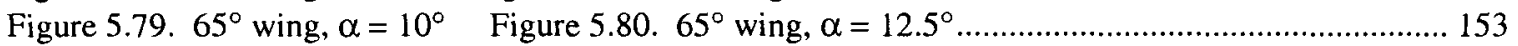

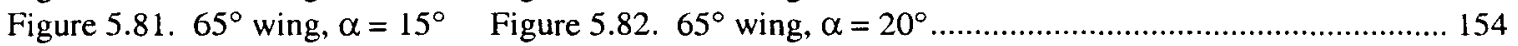

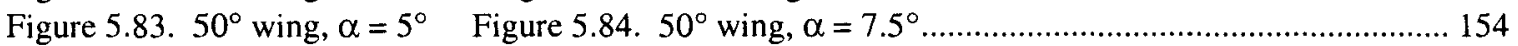

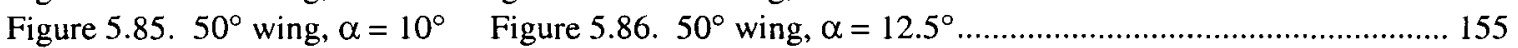

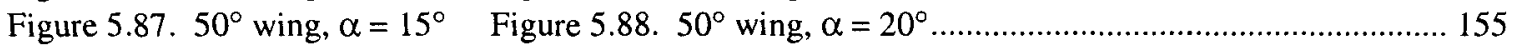

Figure 5.89. $65^{\circ}$ wing, $\alpha=15^{\circ}$, mean 3-component vorticity field................................................. 157 
Figure 5.90. $65^{\circ}$ wing, $\alpha=15^{\circ}$, mean 3-component vorticity field, top view................................... 157

Figure 5.91. $50^{\circ}$ wing, $\alpha=10^{\circ}$, mean 3-component vorticity field.............................................. 158

Figure 5.92. $50^{\circ}$ wing, $\alpha=10^{\circ}$, mean 3-component vorticity field, top view................................... 158

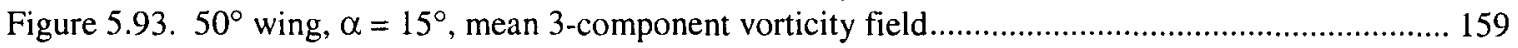

Figure 5.94. $50^{\circ}$ wing, $\alpha=15^{\circ}$, mean 3-component vorticity field, top view .................................... 159

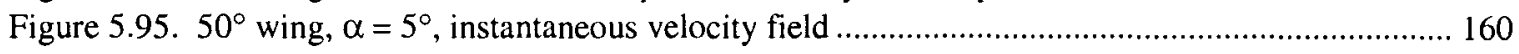

Figure 5.96. $50^{\circ}$ wing, $\alpha=5^{\circ}$, mean and rms velocity fields ........................................................ 161

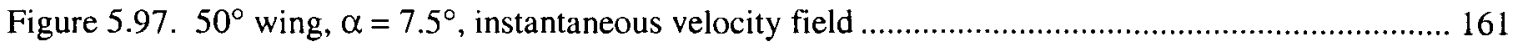

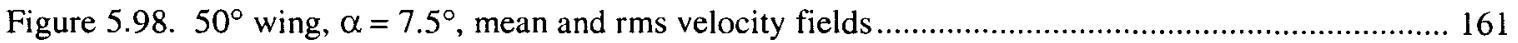

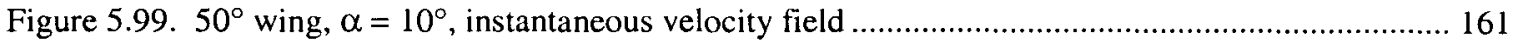

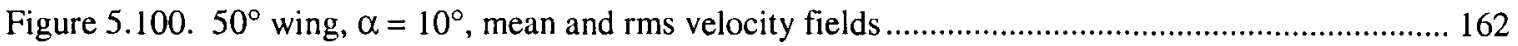

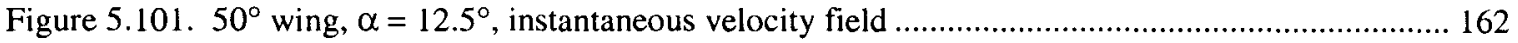

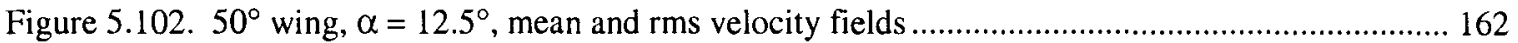

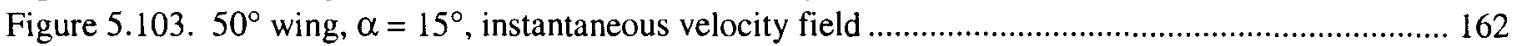

Figure 5.104. $50^{\circ}$ wing, $\alpha=15^{\circ}$, mean and rms velocity fields ................................................... 163

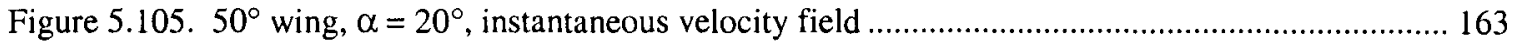

Figure 5.106. $50^{\circ}$ wing, $\alpha=20^{\circ}$, mean and rms velocity fields ................................................... 163

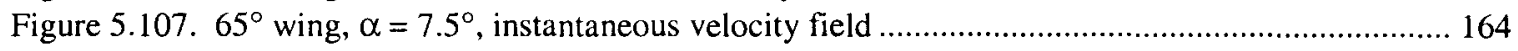

Figure 5.108. $65^{\circ}$ wing, $\alpha=7.5^{\circ}$, mean and rms velocity fields ....................................................... 164

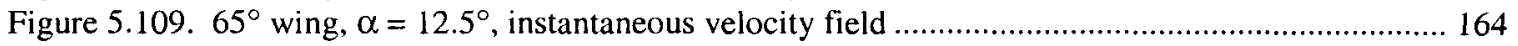

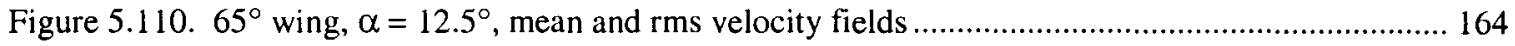

Figure 5.111. $65^{\circ}$ wing, $\alpha=20^{\circ}$, instantaneous velocity field ...................................................... 165

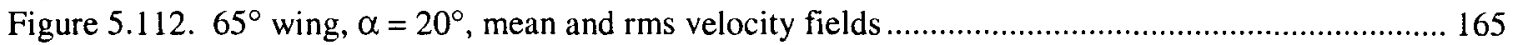

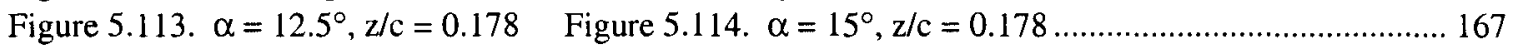

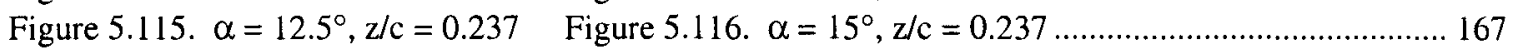

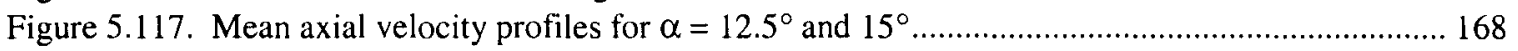

Figure 6.1. LEV core normalized axial velocity, $50^{\circ}$ and $65^{\circ}$ wings ............................................. 175

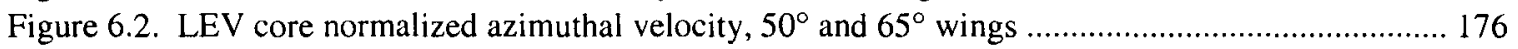

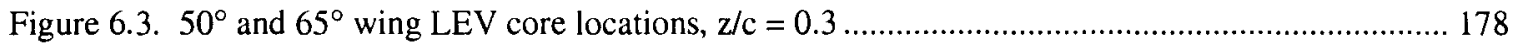

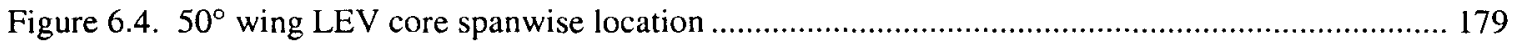

Figure 6.5. Predicted LEV normalized core location vs. LE sweep ................................................... 179

Figure 6.6. Predicted core location vs. experiment....................................................................... 180

Figure 6.7. Comparison of normalized spanwise core location with model of Smith ........................... 182

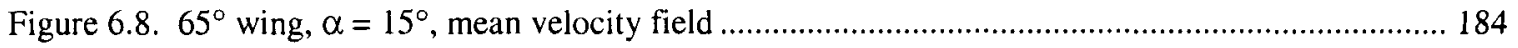

Figure 6.9. $65^{\circ}$ wing, $\alpha=15^{\circ}$, mean velocity field, top view ..................................................... 184

Figure 6.10. $65^{\circ}$ wing normalized velocity profiles for two different Reynolds numbers....................... 185

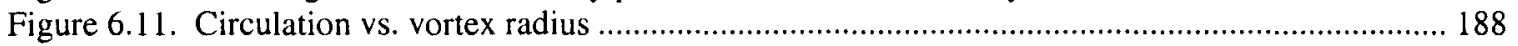

Figure 6.12. Averaged normalized core velocity and LEV radius vs. AOA......................................... 190

Figure 6.13. LEV strength vs. Hemsch and Luckring prediction ........................................................ 193

Figure 6.14. Estimated windward boundary layer displacement thickness ............................................ 195

Figure A.1. Test Section Flow Speed vs. Pump RPM …............................................................... 210

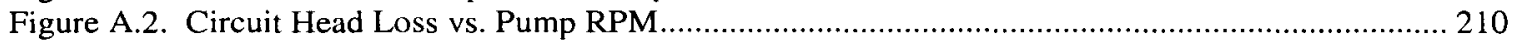

Figure A.3 . Conceptual sketch of free surface "sloshing" ................................................................. 213

Figure A.4. Time traces of free stream velocities, with sloshing dampened ....................................... 213

Figure A.5. Time traces of free stream velocity, with sloshing ........................................................ 214

Figure B.6. Pure out-of-plane artificial displacement.......................................................................... 217

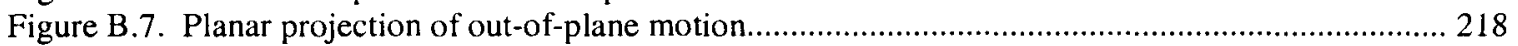

Figure B.8. Left-right planar translation of artificial particle speckling ........................................... 219

Figure B.9. Left-right translation, with 100x magnification of Z-axis ............................................... 219

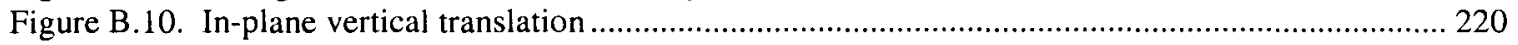

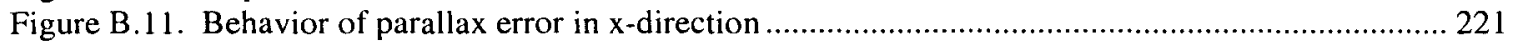

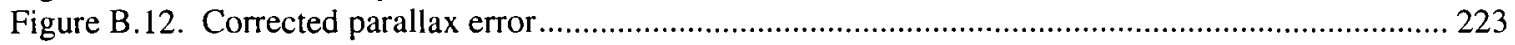

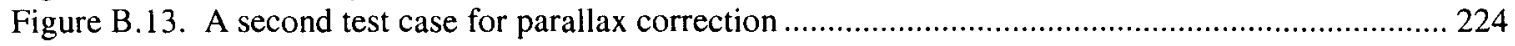

Figure B.14. Correction of parallax error in second test case........................................................... 224 


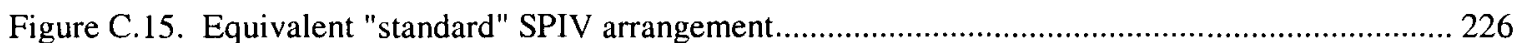

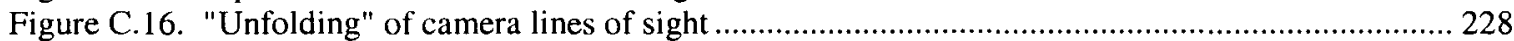

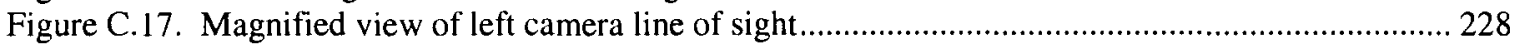




\section{List of Tables}

Table 4-1. Flow visualization test matrix

Table 5-1 . Stereo PIV test matrix; angle of attack sweep ............................................................... 117

Table 5-2. Stereo PIV test matrix, nondimensionalized locations of interrogation planes ....................... 119

Table 6-1. Evaluations of Wedemeyer's profile parameter... 


\section{List of Symbols}

\section{General Aeronautics}

$\alpha$

$A R$

$b$

$c$

$C_{L}$

$\Lambda$

$S$

$t^{*}$

$t$

$\mathrm{z} / \mathrm{c}$

$\Gamma$

\section{PIV Interrogation Plane-Frame Coordinate System}

$\mathrm{X}$

y

$\mathrm{z}$

$\mathrm{X}$

Y

Z

Tunnel-Frame Coordinate System angle of attack, also occasionally abbreviated "AOA"

wing aspect ratio, $=b^{2} / S$

wing span, measured at the trailing edge

wing root chord

wing lift coefficient

delta wing leading edge sweepback angle

wing planform area

convective time, based on $c$ and $U_{\infty}$

nondimensional time, given in multiples of $t^{*}$

nondimensional downstream distance from wing apex

circulation, implicitly normalized by $U_{\infty}$ and $b$

lateral coordinate, positive to tunnel test section starboard vertical coordinate, positive toward tunnel free surface longitudinal coordinate, positive in free stream flow direction

lateral coordinate, positive to wing starboard side vertical coordinate, positive above wing leeward side longitudinal coordinate, positive in free stream direction

\section{Velocimetry Camera Nomenclature}

$\alpha_{L}, \alpha_{R}, \beta_{L}, \beta_{R} \quad$ camera-to-interrogation-plane viewing angles

$C R, C L$

$u_{L}, v_{L}, u_{R}, v_{R}$ subscripts denoting [camera] left (right)

apparent planar velocities recorded by left (right) camera

Velocities

$U_{\infty}$

free stream velocity in water tunnel test section, $\mathrm{mm} / \mathrm{s}$; also $U_{-}$inf

$U_{z}$ velocity normal to the interrogation plane; also LEV core axial velocity 
$U_{\theta} \quad$ azimuthal velocity in the primary LEV cross-section

V vertical velocity in PIV interrogation plane, taken along spanwise cut through LEV; sometimes interchanged with $U_{\theta}$

Other Symbols

E

Stereo PIV in-plane parallax error; also amalgamation of the various experimental errors

$K_{M S}$

Mangler and Smith similarity parameter

$R_{0}$

LEV radius corresponding to peak circulation value

$K_{W}$

Wedemeyer's "profile" parameter

$a_{S}$

Smith's similarity parameter

Some Frequently Used Abbreviations

AOA

BL

LE, TE

LEV

$\operatorname{Re}$

SPIV

VB angle of attack

boundary layer

leading (trailing) edge of the wing leading edge vortex, primary or secondary

Reynolds number, based on $c$ and $U_{\infty}$ stereo particle image velocimetry; also SDPIV and stereo PIV vortex breakdown, or vortex burst 


\section{Chapter 1 Introduction}

\subsection{Historical background}

While recent times have seen abatement in the development of new airframe configuration aerodynamics, seminal advancements of the past century continue to undergo refinements. One such advancement was the shift away from the classical notion that an efficient flight vehicle must necessarily have an attached flow field. That is, the streamline pattern can be allowed to appreciably deviate from a pattern nominally following the surface of the aircraft or its components, so long as this deviation is steady, controllable and predictable. One of the most widely encountered and extensively studied examples of such a separated flow is that about a delta wing with sharp leading edges.

The delta wing has a planform shape of an isosceles triangle, evidently deriving its name from its resemblance to the Greek letter $\Delta$. The equal-length sides of the triangle form the wing leading edges (LE), and the base forms the trailing edge (TE). Such wings and their variants have a long history in aeronautical engineering. Delta wings were proposed some six decades ago as a solution to problems of supersonic flight. Lippisch $^{1}$ was evidently the first to design an airplane with a delta wing, in 1945.

The aerodynamic onset of supersonic effects is largely driven by the Mach number of the flow relative to the leading edge, rather than that of the free stream, whence it follows that high sweepback delays the undesirable effects of shock waves; namely, wave drag $\left(\mathrm{Jones}^{2}\right.$ ). The effect is especially important when the sweep angle of the leading edges is greater than the sweep of the Mach cone emanating from the wing 
apex. This consideration has historically been responsible for the predominant attention to delta wings of large sweep.

Also, the shift of the aerodynamic center of the wing in going from subsonic to supersonic flight is more gradual for delta wings than for nominally rectangular (or elliptical) wings ${ }^{3}$. The combination of low aspect ratio and large taper, meanwhile, have important structural and packaging advantages for the airplane designer. They allow for small wing section thickness ratio without excessive sacrifice in bending and torsional strength, while conveniently maintaining ample internal volume near the wing root. Wing-fuselage blending is also very natural with this kind of configuration. Indeed, a number of the first generation of supersonic aircraft used delta wings with success. While most military fighter aircraft of the present generation have largely abandoned the pure delta-type configurations of the first generation supersonic fighters, in light of issues such as wing tip stall and wing-empennage coupling, some examples of the latest generation of designs are moving back toward such configurations, albeit with rounded leading edge shape. These include the Eurofighter (leading edge sweep of $53^{\circ}$ ) and Dassault Rafale (leading edge sweep of $50^{\circ}$ ). Curiously, these wings are all of moderate sweep.

But the most interesting features of delta wing flow occur in incompressible flow, and can even be observed in water. More precisely, they are independent of the Mach number, from $\mathrm{M}=0$ well into the supersonic range, as demonstrated by various authors (e.g., Stanbrook and Squire ${ }^{4}$ ). Already in the 1950's it was observed that at moderate to high angles of attack, delta wings with sharp leading edges can not maintain a fully attached flow. Intuitively, this was considered a disadvantage, both in terms of subsonic 
lift and drag. Yet, in the case of sufficiently slender delta wings, the flow separation over the leading edges results in a net suction over significant portions of the leeward (i.e., upper) surface of the planform, hence assisting in the overall aerodynamic lift, rather than detracting from it. In going over the leading edges of the wing, from the windward side to the leeward, the windward boundary layer separates, and is shed as a free shear layer. This layer then rolls up into what eventually forms a tight vortex core, one on each side of the wing. The relatively poor aerodynamic efficiency of such low aspect ratio wings is in fact improved by this separation.

Initially, the beneficial effects of leading edge separation were viewed primarily as a means of improving the takeoff and landing performance of high speed, high wing loading aircraft. But since at least the 1970's, there has been interest in so-called supermaneuverability (i.e., post-stall controllability) of military fighter aircraft, with configurations based on the delta wing (see e.g., Herbst ${ }^{5}$ ), where the objective was to maintain control of the aircraft at high angles of attack. At sufficiently high angles of attack - say, $30^{\circ}$ - separation is probably unavoidable with any [passive] wing. But if the separation can be managed, flight is still possible. It is at these angles of attack that configurations based on delta wings found their most useful application. Typical of these configurations is the strake-delta combination, where a very high sweep wing "root" precedes a more gradually swept main element of much higher aspect ratio. Like the regular delta wing, these develop a "vortex lift" due to organized, regular separated flow structures due to flow separation at the leading edges (see Section 1.2.1 below). Motivations for this design strategy are described by Polhamus ${ }^{6}$. Another review, current to 1976 , is given by Parker ${ }^{7}$. 
Polhamus also points out that again for reasons of reducing supersonic wave drag, the delta wing leading edges were made sharp. But then it was observed that sharp leading edges actually strengthen the "vortical lift effect," because they tend to fix the separation right at the leading edges. To this day, the optimal shaping of the leading edge - to balance considerations of attached flow at low angles of attack vs. fixed separation at high angles of attack, low viscous drag at subsonic speeds vs. low wave drag at supersonic speeds, etc. - is still a matter of some controversy.

The "post stall" regime of flight depends on auspicious management of the welldefined regions of separated flow - namely, the leading edge vortices. The collapse of the favorable pressure field set up by these vortices, due to a stall-like process referred to as vortex breakdown, can have catastrophic effects on the wing performance. Vortex breakdown has a highly complex time dependence, and presents numerous difficulties in aircraft stability and control.

Kuechemann ${ }^{8}$ notes that aeronautical designs based on delta wings possess the essential engineering criteria of stable, controllable, and efficient flow, but possibly not for wings of only moderate sweep. Considering delta wings of increasing sweep, there is a fundamental contradiction between better high angle of attack controllability (higher sweep) and higher lift to drag ratio (lower sweep). Clearly, a lower sweep/higher aspect ratio wing will have stronger circulation in the lifting line sense - and thus, a steeper lift curve slope. Thus, a wing of moderate sweep is naturally of interest. Unfortunately, such wings are more difficult to analyze theoretically, and are not as useful for supersonic applications. Thus, they have received appreciably less attention than highly swept wings. 
The vast literature on delta wing flow physics is comparatively silent on the basic issue of the transition from the slender delta wing vortical flow, to the separated flow of a high aspect ratio classical wing, which is largely devoid of the coherent, stable separated flow structures useful for aeronautical applications. The present work considers this issue, in the context of the admittedly old problem of a sharp-edged delta wing in incompressible, steady flow. A variant of a relatively new optical diagnostic technique, generally used for more abstract problems, is adapted to the present experimental setup. The primary method of investigation is experimental flow diagnostics with stereo Digital Particle Imaging Velocimetry, (SDPIV), which produces data for all three velocity components in planar slices. An abstracted model of a well-studied configuration and a less frequently encountered geometry of lower sweep are examined with this technique. The experiments were conducted at low Reynolds numbers - a fact that was found to be important only a posteriori.

In the following, the physics of incompressible steady flow about a delta wing is described in more detail. The aerodynamic performance of delta wings is reviewed, with particular emphasis on wings of moderate sweep, also referred to as nonslender wings. A selection of theoretical and experimental results is mentioned, with motivations for the techniques applied in the present study.

\subsection{A resume of delta wing flow features}

The flow pattern about a delta wings is quite complex. In most cases, it is dominated by separated flow emanating from the leading edges, generally forming a pair of coherent vortical structures called leading edge vortices. These structures are observed over a large range of Reynolds numbers, from at least $O\left(10^{4}\right)$ to arbitrarily high values. 
While the present investigation focuses on wings with "sharp" leading edges (loosely speaking, the leading edge radius is less than the local boundary layer thickness), wings with appreciable leading edge radius can still produce coherent leading edge vortices, albeit of reduced amplitude and in a narrower angle of attack range (see for example Kegelman and Roos $\left.{ }^{9}\right)$.

\subsubsection{Leading edge separation and shear layer rollup}

A schematic representation of established LEV flow about the leeward side of a relatively slender delta wing with windward LE bevel is given below ${ }^{10}$. The schematic is a cut along a "crossflow plane" - that is, a plane perpendicular to the nominal free stream flow direction. In this representation, trailing edge and wake effects are not included.

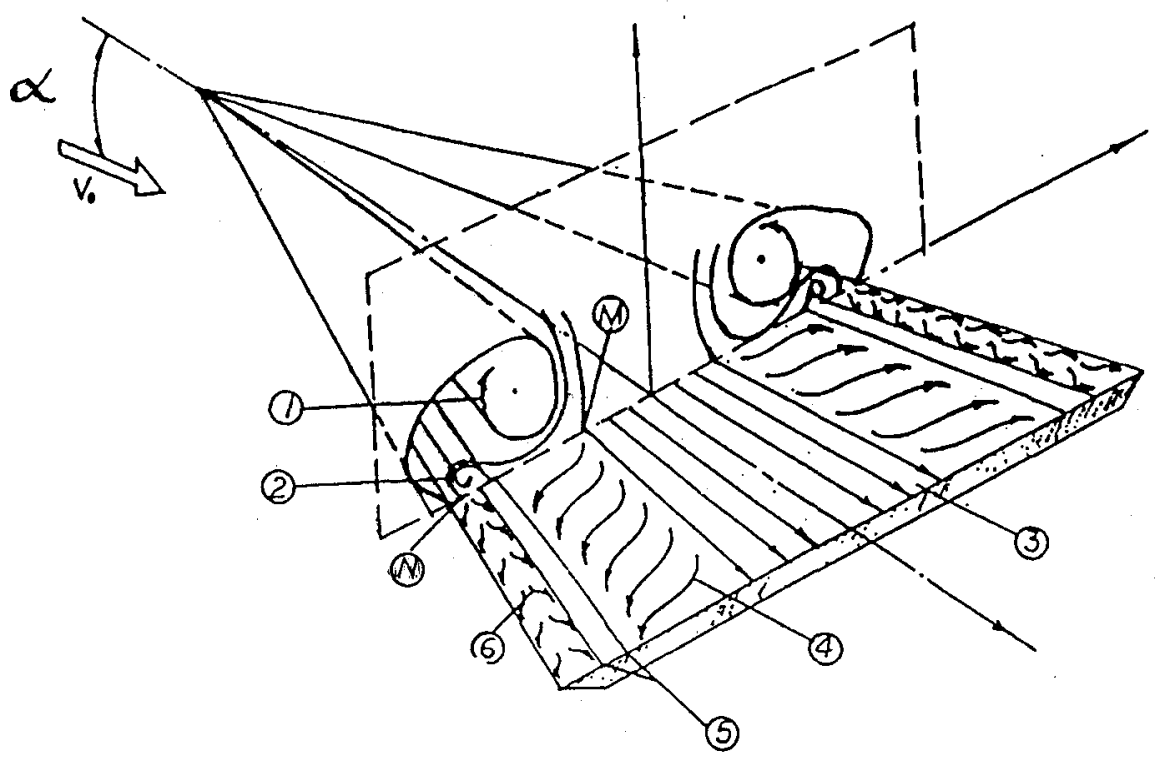

Figure 1.1. Schematic of the delta wing flow field (based on Hoerner ${ }^{10}$ )

In Figure 1.1, the wing is a flat plate with sharp leading edges and windward-side (i.e., lower surface) bevels. The indicated elements are:

1) LE shear layer rolling up into primary leading edge vortex (LEV) 
2) Secondary vortex

3) Nominally axial (attached) flow in center region

4) Flow outboard toward secondary separation line

5) Separation/attachment lines

6) Outboard flow near leading edge

(1) and (2) are represented as rolling-up shear layers of very small thickness. In reality, their thickness need not be small, and in fact is of comparable size to the thickness of the windward-side boundary layer just prior to separation at the leading edges. The shear layer thickness varies with Reynolds number, as does the boundary layer. (1) forms the leading edge vortex proper, when the "winding sheet" is sufficiently tight that viscous diffusion produces a spatially smeared sheath of vorticity. In the case of the present study, the Reynolds number is sufficiently low that no more than half a revolution of the shear layer rollup is necessary to pass to this vortical region. Its distribution of axial vorticity is akin to a Rankine vortex in two dimensions, though threedimensional effects can in general not be ignored.

The outboard flow ((4) and (6)) shown in the above figure also has a strong dependence on Reynolds number. As will be seen from the results of Chapter 6, flow in this region need not necessarily display the smooth organization implied in Figure 1.1, and can instead be separated.

$\mathrm{M}$ is the reattachment line of the primary separation, which bounds the threedimensional surface dividing the nominally attached flow along the wing center region, and the flow drawn into the outboard vortical structures. Inboard of the port and starboard M surfaces, the flow (3) is attached and follows the surface of the wing. $N$ is the reattachment line of the secondary separation. The term "secondary leading edge vortex" is of some ambiguity, as this vortex does not, strictly speaking, emanate from the 
leading edge. But it will be referred to as such, because its ultimate origin is due to the effects of LE separation, and in keeping with the convention in the literature. The secondary vortices are essentially boundary layer phenomena. Referring to the situation in a crossflow plane, as the primary LEV induces an adverse pressure gradient in the leeward side boundary layer, in going in the outboard direction. The boundary layer then tends to separate, erupting in its own shear layer, which then rolls up under selfinduction, much like the original shear layer at the leading edge. This rollup results in the secondary LEV. A tertiary or even higher order separation can result from a continuation of this process, though in practice, viscous diffusion attenuates gradients between the various structures and terminates the formation of higher order separation for flight-scale Reynolds numbers. Tertiary separation is generally not observed for water tunnel-scale Reynolds numbers, such as in the case of the present experiment.

Additional insight can be obtained from considering the near wake of the wing, as shown in a schematic sense in Figure $1.2^{8}$.

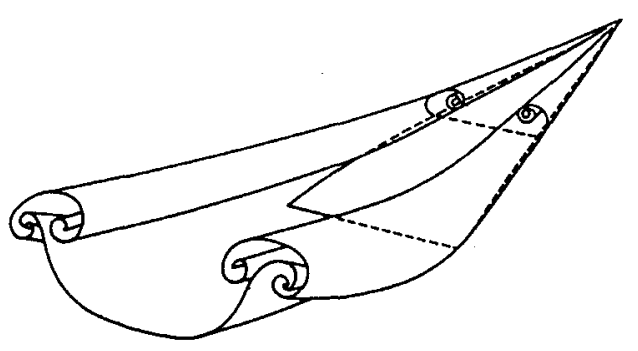

\section{Figure 1.2. Delta wing shear layer rollup in near wake (from ${ }^{8}$ )}

The leading edge shear layer interacts with the trailing edge shear layer to form counter-rotating vortex sheet pairs at each wing tip. The wake of the wing deviates strongly from planar. The nonplanar wake evidently introduces more downward momentum into the flow, thus producing more lift that would have been possible with a 
strictly attached flow over the wing. This is one approach to the explanation of the additional lift increment due to the LEVs, and raises issues regarding the role of potential lift due to attached flow and a trailing edge Kutta condition vs. "vortical flow" due to LEVs. Curiously, the trailing edge vortex sheet is seen to commence winding on its spanwise boundaries in the same rotational sense as the secondary vortices over the wing leeward surface.

As will be seen subsequently, the fine detail of these flow features varies considerably with Reynolds number, while the gross features of leading edge separation and shear layer rollup are largely invariant.

\subsubsection{Conical flow}

The triangular shape of delta wings, the lack of a discernable streamwise length scale, and the dominance of crossflow-plane pressure gradients (vs. the streamwise pressure gradient) for slender delta wings leads to the observation that the streamline pattern in succeeding downstream crossflow planes scales in linear proportion with the local span. Thus, the flow evolves "conically," an analogy to the "cylindrical" flow used to describe a 2-D airfoil. Experiments have shown that for a slender wing, such as of aspect ratio 1.0 , velocity field is indeed approximately conical near the wing apex, but deviates from a conical distribution in approaching the trailing edge ${ }^{81}$. Intuitively, it appears that for less slender wings, the flow field must deviate further from the conical pattern.

\subsubsection{Application of flow topology}


Complicated flow structures, such as three-dimensional separations, have to some extent been amenable to study of their "topology" - that is, the accounting of certain quantitatively identifiable characteristics that remain invariant in going between flows of one family, but which change in going from one flow family to another. These characteristics are principally the points, curves, or (in 3-D) surfaces of separation or attachment. In on-surface flow visualization, one considers the pattern of "skin friction lines," the on-surface analogue of streamlines, which reveal the presence of separation and attachment of the flow from the body surface. In the present investigation, onsurface flow visualization was not attempted. The off-surface methods - both the flow visualization and the quantitative velocimetry - can also to some extent benefit from an appeal to topological concepts, for example by counting the number and type of singularities, and asking if the "conservation equations" for these singularities are satisfied.

In the crossflow plane, the topology of the dividing streamlines for a canonical case is given in the following figure (adapted from Verhaagen ${ }^{11}$ )]

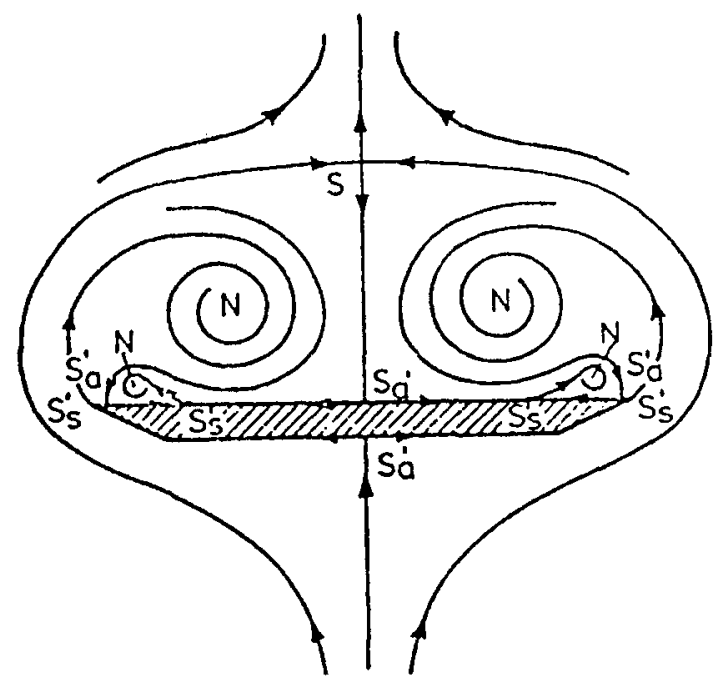

Figure 1.3. Crossflow plane topology 
Here, $S$ is a saddle point, with the subscripts $s$ and $a$ standing for separation and attachment, respectively. The prime symbol (') refers to a so-called half-saddle - a saddle point on the body surface. $N$ is a [focal] node. Separation at the leading edge is represented by $S_{S}^{\prime}$. The primary and secondary LEVs have "centers" corresponding to focal nodes, $N$. The separation from which the secondary LEVs emanate is also associated with a half-saddle of separation, $S_{S}^{\prime}$. Windward and leeward stagnation lines are associated with half-saddles of attachment, $S_{A}^{\prime}$.

The terminology is borrowed from the phase-plane theory of ordinary differential equations (see for example Rom ${ }^{12}$ ). In the present work, use of topological concepts will be limited to casual description of velocity vector and streamline plots.

\subsubsection{Vortex breakdown}

Stable, well-defined leading edge vortices are not sustainable for arbitrarily high angles of attack. Eventually they undergo vortex breakdown (VB), which is qualitatively analogous to the stall of a high aspect ratio wing. While a precise definition of VB remains elusive, VB is generally associated with a sudden transition from tightly-coiled rotating flow with straight trajectory and high axial speed, to broadened, retarded flow with much lower peak vorticity. The types of breakdown of principal significance to delta wings have been loosely grouped into two categories: "bubble" breakdown, where the LEV core reaches a stagnation point, and "spiral" breakdown, where the flow does not actually stagnate but winds around in a bulk motion akin to a corkscrew (Sarpkaya ${ }^{13}$ ). Breakdown has received extensive attention in the recent literature (see for example the review by $\mathrm{Visbal}^{14}$ ). Issues include the structure of the VB region (Towfighi and Rockwell ${ }^{15}$ ), response of VB to unsteady boundary conditions such as pitching or rolling 
of the delta wing, and prediction of breakdown chordwise location as a function of sweep and angle of attack. In the present investigation, the physics of VB itself have not been considered. Rather, of interest was the effect of the LEV instability attributed to VB on the velocity field of the wing, and hence on its stall behavior.

Generally speaking, VB is found to occur further upstream for wings of lower sweep (at a given angle of attack), and at increasing angle of attack (for a wing of given sweep). But curiously, VB appears to depend strongly on numerous factors of the wing geometry, testing condition, etc., quite beyond the primary parameters of LE sweep and angle of attack. An example is given by Jobe ${ }^{16}$, for wings of $65^{\circ}$ sweep (Figure 1.4; references in the figure are those of the source paper).

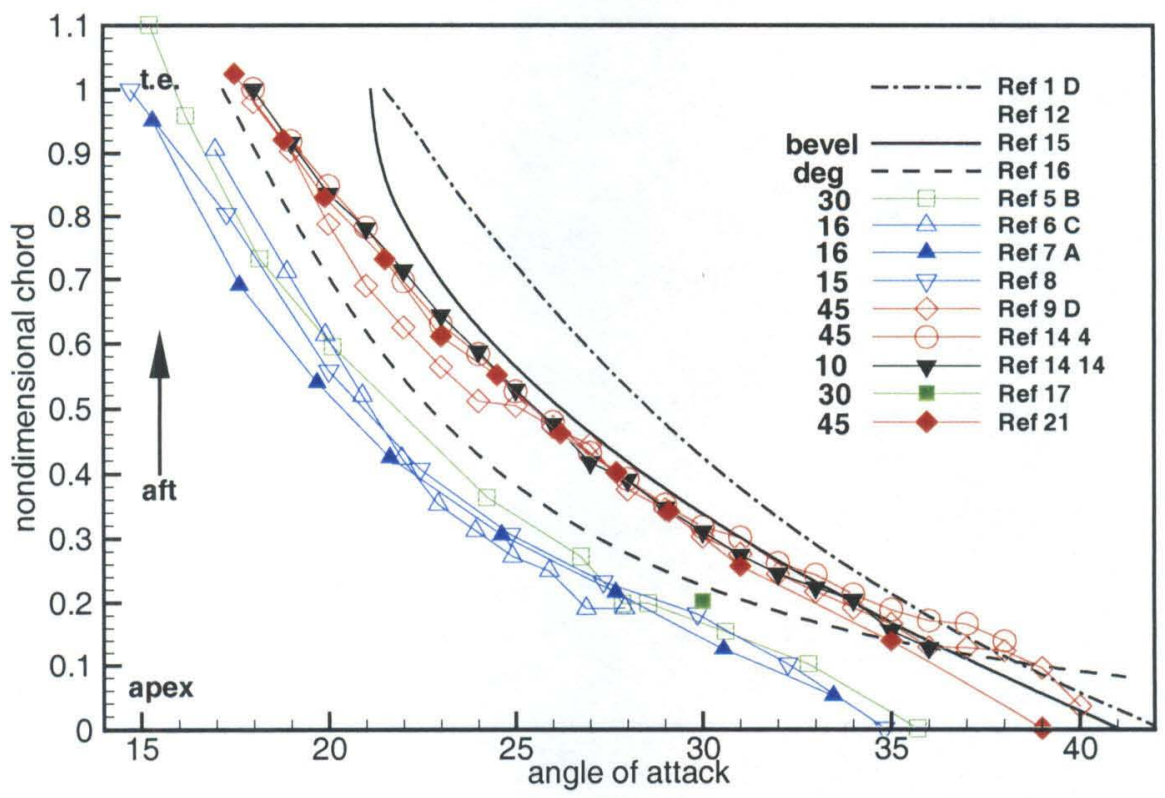

Figure 1.4. Vortex breakdown locations for various $65^{\circ}$ wings

For instance, at $25^{\circ}$ angle of attack, the VB point can lie anywhere from one-third to two-thirds of the root chord behind the wing apex, depending to which experiment one refers. This and other aspects of the flowfield data result in a curious situation where the bulk features of the flow are well known, but the details, such as vortex core trajectory, 
VB location, presence and strength of secondary LEVs, etc., remain essentially open questions. This is exacerbated for the case of nonslender delta wings.

It is worth noting in passing that the flow topology becomes spectacularly complex in the vicinity and downstream of $\mathrm{VB}^{17}$.

\subsection{Delta wing performance}

We next consider characteristics of delta wings from the aerodynamicist's point of view. In this regard, the lift, drag, and stability characteristics of the wing are more important than the particular details of its flowfield.

\subsubsection{Lift and drag characteristics}

Leading edge sweep of course affects aspect ratio, according to the relation

$$
A R=4 \cot (\Lambda)
$$

eqn. $1-1$

$\Lambda$ is the sweep of the leading edge. In what follows, the wing geometry will be referred to according to this sweep angle.

Generally, lift curve slope decreases with decreasing aspect ratio, and the induced drag increases. This is a fair statement for wings of any planform shape. But the effect is not necessarily beyond doubt. A plot of the lift curve for various sweep angles is given in Figure 1.5 below (from Wentz and Kohlman ${ }^{18}$ ). 


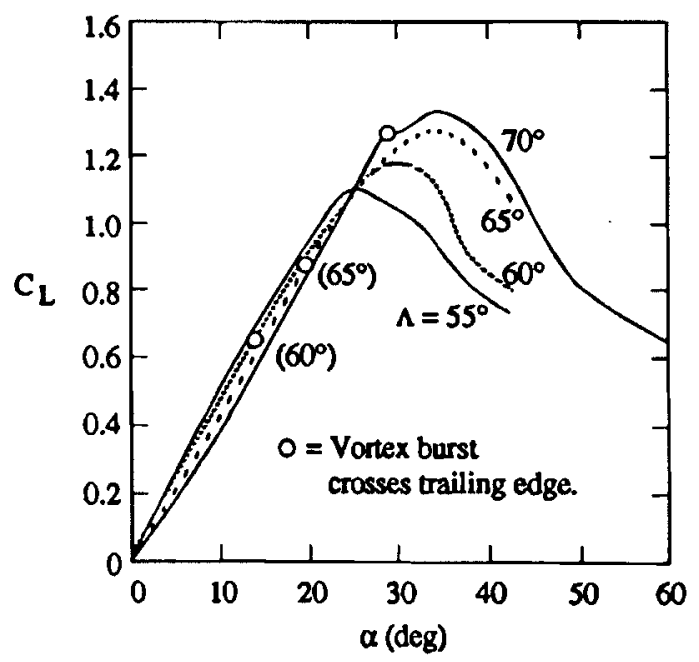

Figure 1.5. Lift curves of some delta wings ${ }^{18}$

Curiously, below $20^{\circ}$ angle of attack, the curves for all of these wings are fairly close to one another. Figure 1.5 also captures a trend observed in numerous subsequent investigations, that the lift coefficient continues to increase for angles of attack well beyond those where the VB first crosses the trailing edge.

A similar conclusion is apparent from considering some data for drag polars for wings of different sweep, such as Figure 1.6 (from Earnshaw and Lawford ${ }^{19}$ )

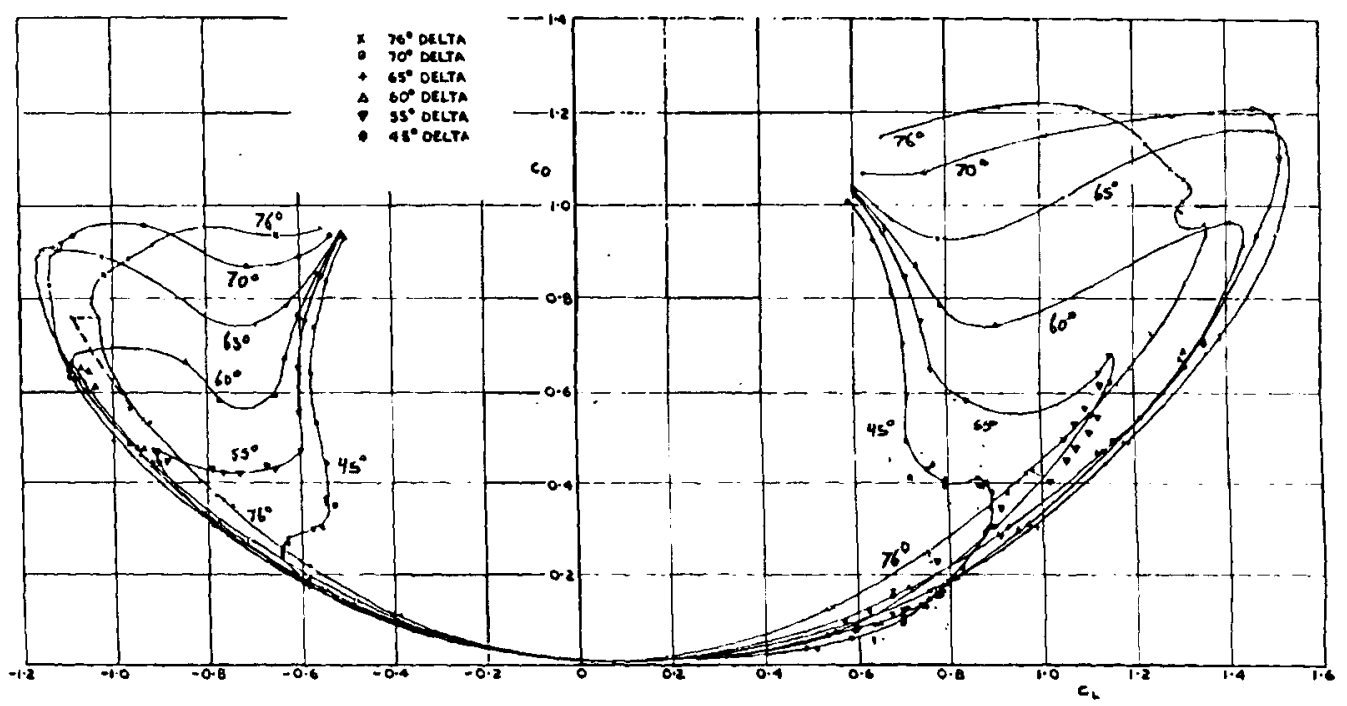

Figure 1.6. Drag polar for delta wings of 45 to 76 degree sweep $^{19}$ 
Earnshaw and Lawford ${ }^{19}$ conducted flow visualization and force balance tests on wings of $76^{\circ}, 70^{\circ}, 65^{\circ}, 60^{\circ}, 55^{\circ}$, and $45^{\circ}$ sweep. These wings had an effective camber, whence zero lift does not occur at zero angle of attack and the drag polars are not symmetric. While the scaling of the plot is somewhat deceptive, it is nevertheless apparent that at the lower angles of attack, the drag polars of the $50^{\circ}-70^{\circ}$ wings are not greatly different - a phenomenon consistent with the lift curve behavior. The $45^{\circ}$ and $76^{\circ}$ wings are outlying cases. For the three least swept wings, they found at best marginal evidence of coherent LEVs, and none at all for the $45^{\circ}$ wing.

The wings of lowest sweep have marginally better drag polar at low angles of attack, but lose the "high angle of attack delta wing property" of delayed stall due to LEV suction.

Thus, it is seen that for performance reasons, wings with pointed tips (taper ratio of zero) and moderate sweep - the nonslender delta wings - are not frequently encountered in aerodynamic design. This work addresses such wings mostly from the academic interest of considering the transition in flow behavior between wings of low and high sweep.

\subsubsection{A baseline "slender" configuration}

In recent years, the delta wing of $65^{\circ}$ leading edge sweep has received particular attention, as a compromise in the competing benefits of higher and lower aspect ratios (see for example the review by Jenkins and Hanff ${ }^{20}$ ). Figure 1.6 is certainly interesting evidence that of all the different sweep wings tested, the $65^{\circ}$ wing has the best 
compromise of low induced drag and very good stall resistance - at least under those particular testing conditions.

The lift curve slope of a $65^{\circ}$ sweep delta wing discussed in ${ }^{20}$ is given in Figure 1.7. A drawing of the model referred to in ${ }^{20}$ is given in Figure 1.8.

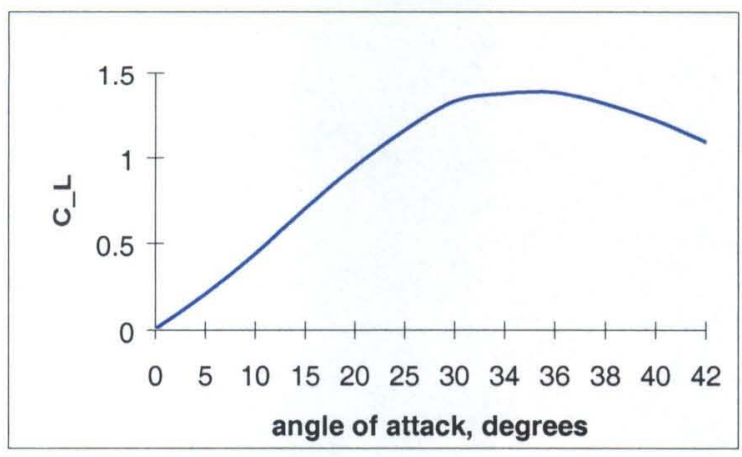

Figure 1.7. Lift curve of IAR/WL delta wing

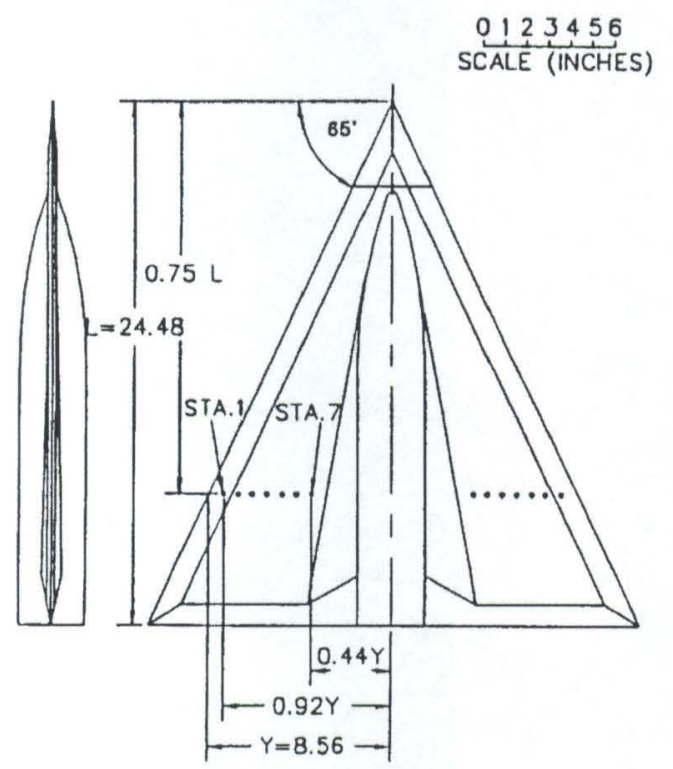

Figure 1.8. IAR/WL $65^{\circ}$ delta wing model

The IAR/WL wing has a centerbody and symmetrical bevels. In the present study, a more abstracted $65^{\circ}$ wing was used as baseline "slender wing" case. The choice of the actual wing geometry was motivated by considerations of simplifying the experimental procedure, as discussed further in Chapter 4. 
Comparing the breakdown location results of Figure 1.4 and the lift results of Figure 1.7, it is seen that peak lift coefficient is attained when VB is almost at the wing apex; that is, at approximately $20 \%$ of the root chord. Extrapolating this result to wings of yet lower sweep, one might say that the loss of coherent LEVs does not necessarily lead to a drop in lift coefficient.

Hemsch and Luckring ${ }^{21}$ point out that LEV strength, and consequently vortex lift, actually decrease with increasing leading edge sweep angle-provided that VB does not occur. Of course, potential lift obviously decreases with increasing sweep as well. So it is not strictly correct to say that the LEVs of nonslender wings are weak, and therefore prone to instability.

\subsection{Effects of leading edge sweep}

Su et al. ${ }^{22}$ applied topological concepts to categorize the delta wing flowfield into nine types (Figure 1.9), depending on sweep angle and angle of attack, based on interpretations of on-surface flow visualizations.

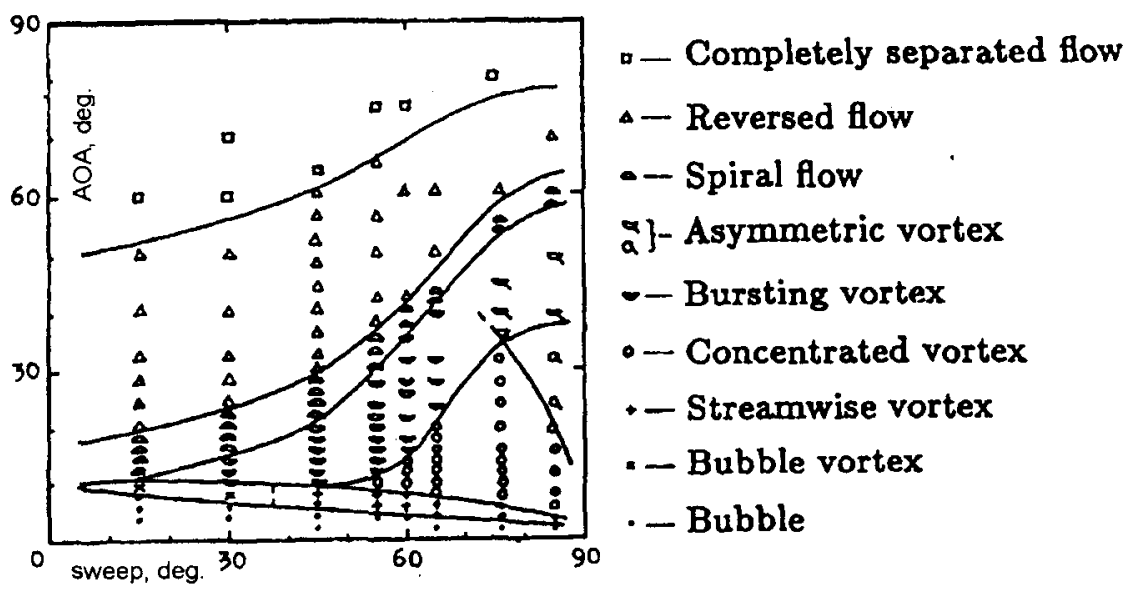

Figure 1.9. Nine types of flowfields of delta wings (from Su et al. ${ }^{22}$ ) 
In the caption of Figure 1.9, the top three entries can be considered "blanket separation" with the wing essentially stalled; the next three have at least partially coherent LEVs, and the last three represent a "pre-LEV" state where the leading edge shear layer evidently does not separate at all, or else, does not roll up into a coherent vortex over each wing panel. Regarding the latter, Su et al. report a "3D bubble near the LE" (the last of the cases in the caption for Figure 1.9), evidently for wings of e.g., $45^{\circ}$ sweep, with no rollup into a LEV. For larger sweep, they report an array of streamwise vortices about the leading edges at low angles of attack, which only coalesce into a recognizable LEV at higher angles of attack. As will be seen in Chapters 5 and 6, the results of the present study are rather different. LEVs were observed down to arbitrarily small angles of attack. At no time were a leading edge bubble or arrays of streamwise vortices observed.

The limit of decreasing leading edge sweep is the rectangular wing. Indeed, some benefit can be derived from making the comparison with rectangular wings of small aspect ratio. For a rectangular wing of aspect ratio of 2 , "the vorticity produced is shed as a shear layer surrounding the periphery [along every edge] of the wing"(Freymuth ${ }^{23}$ ) at the higher angles of attack, akin to the situation with the delta wing (see Figure 1.2). But at the lower angles of attack, say on the order of $10^{\circ}$, vorticity is evidently removed by "backdiffusion into wing surfaces in regions of adverse pressure gradient." As the angle of attack is increased, "convective leakage" of vorticity from its "anchor" points (in this case, the leading edge/wing tip junctures), evidently concomitant with the destruction of organized vortical structures, becomes more important, and dominates in going toward 
the high angles of attack. The essential qualitative difference between the rectangular and delta wings is that for the latter, the apex is the vorticity anchor point.

Roos and Kegelman conducted an extensive series of wing tunnel tests of $60^{\circ}$ and $70^{\circ}$ delta wings (see ${ }^{24}$ for a review). Figure $1.10^{24}$ describes another rendition of various types of possible delta wing flowfields, in the $\alpha-\Lambda$ parameter space. This can be compared to Figure 1.9. Of particular interest in Figure 1.10 is the relationship between the streamwise location of VB as a function of sweep and angle of attack. With increasing sweep, the AOA at peak $C_{L}$ increases. Unfortunately, details about wings of sweep below $55^{\circ}$ and $\mathrm{AOA}$ below $20^{\circ}$ are not available.

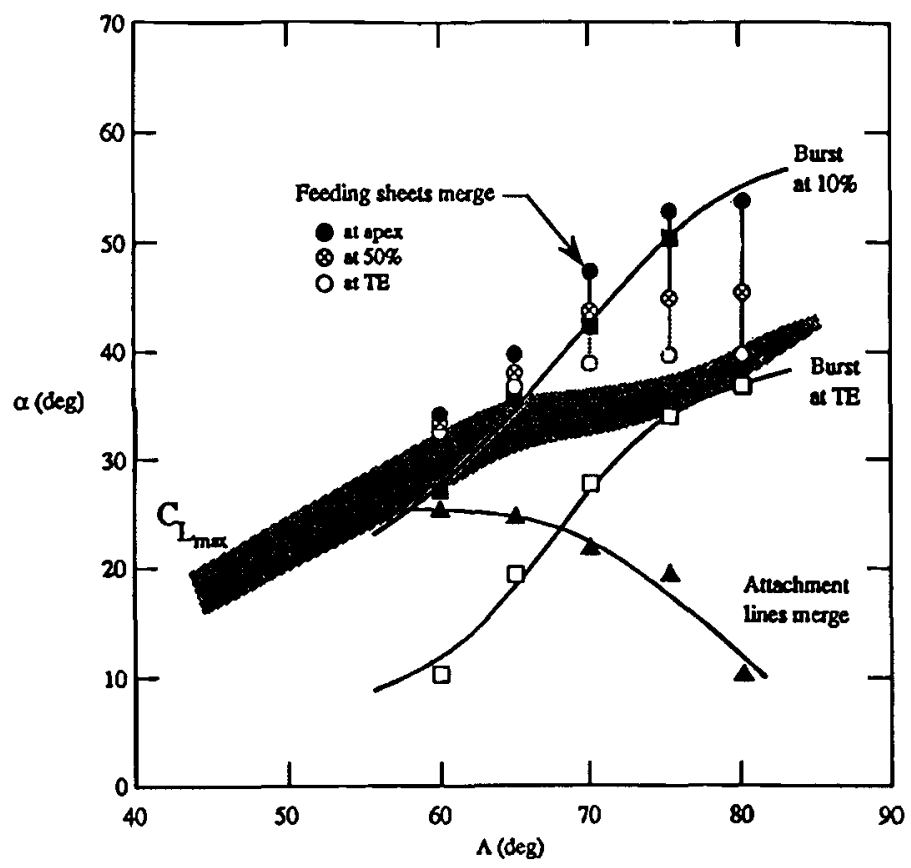

Figure 1.10. Effect of LE sweep angle of delta wing flow features ${ }^{24}$

The flow visualization experiments of Ayoub and McLachlan ${ }^{25}$ identified 5 stages in LEV flow patterns for a wing of aspect ratio 1.0, depending on angle of attack. At low to moderate angles of attack, LEV structure was stable and coherent. The LEVs exhibited unsteadiness in the breakdown location at very high angle of attack, where 1) 
the LEVs were substantially above the wing surface, and thus, LEV/BL and $\mathrm{LEV} /$ secondary interactions would be minor, and 2) at such high AOA, potential flow effects would be minor due to a weakened trailing edge Kutta condition, and hence the pressure field about the wing would be different. This situation is more akin to the shedding behind a flat plate normal to the flow.

\subsection{Implications of delta wing theoretical models}

Efforts at theoretical modeling of the delta wing flow field can be grouped into two broad categories: those that attempt to discern the geometry and structure of the LEVs and the concomitant flow features, and those that predict the wings' aerodynamic coefficients. The availability of the large body of experimental data obtained in the present investigation suggests comparison with representatives of the former class of models, particularly since most such models assume laminar flow when considering viscous effects, and the Reynolds numbers in the present investigation are consistent with a nominally laminar flow. Two particular cases are introduced below, and considered further in Chapter 7. The aerodynamic prediction models are less applicable to the present study, since aerodynamic coefficients were not measured. However, one particular case - the model of Polhamus ${ }^{30}$ - is mentioned for purposes of considering the relative importance of the LEVs for a wing of given sweep.

\subsubsection{The slender wing assumption}

Most theoretical models make the assumption that the delta wing is slender. This assumption is an outgrowth of low aspect ratio, and can be approached in two ways. When the aspect ratio is low, the trailing edge is far away from the apex (in relation to the 
local semi-span), so the wing is viewed as semi-infinite downstream of the apex.

Alternatively, one can remark that the strength of streamwise gradients (in pressure, velocity, etc.) is weaker than that of the crossflow gradients. Thus one can essentially reduce the analysis of the 3-D flowfield into that of crossflow planes, whence the problem is rendered quasi-2D. For instance, in the inviscid approximation, the 3-D Laplace equation loses the streamise-direction term. These two considerations are both integral to the assumption of conical flow.

High aspect ratio wings have small spanwise gradients, and high chordwise gradients. Slender delta wings are the opposite. But wings of moderate sweep would be expected to have a particularly complex 3-D flowfield, with no preferred orientation of dominant gradients. That is an excellent candidate for experimental investigations with a three-dimensional diagnostic technique, especially a field technique such as stereoscopic PIV. Theoretical analysis, however, has largely avoided such cases.

\subsubsection{Vortex core models}

Following the reasoning that it is the vortical lift that is the quintessential characteristic of delta wings, and that this lift results from the rollup of the LEVs into tight cores, it is useful to abstract the entire flow field to that of an isolated vortex core, with suitable core boundary conditions derived from the main flow field. Then the core can be studied separately. In particular, though the entire flow field may or may not be conical, that of the core most likely is. This is especially appropriate at low Re, with the merging of the vortex sheet windings into a "smeared" viscous region. 
The vortex core model of Hall, based on matching asymptotic expansions (inner viscous laminar core, outer conical inviscid flow), has become a benchmark for core velocity profile models ${ }^{26}$. Figure 1.11 and Figure 1.12 show the results of Hall, as well as the wind tunnel results of Earnshaw ${ }^{27}$, for axial and azimuthal velocity distributions, respectively. The wing had aspect ratio $1.0,14.9^{\circ}$ angle of attack, and nominal Reynolds number of 43 million. For the purposes of the present study, these figures have several important consequences:

1) The "inner viscous portion" of the LEV - as characterized by the core radius across which the azimuthal velocity varies appreciably - is small compared to the wing span.

2) While theory overpredicts the peak axial velocity, theory and experiment agree that peak axial velocity in the core exceeds the free stream velocity by at least a factor of two.

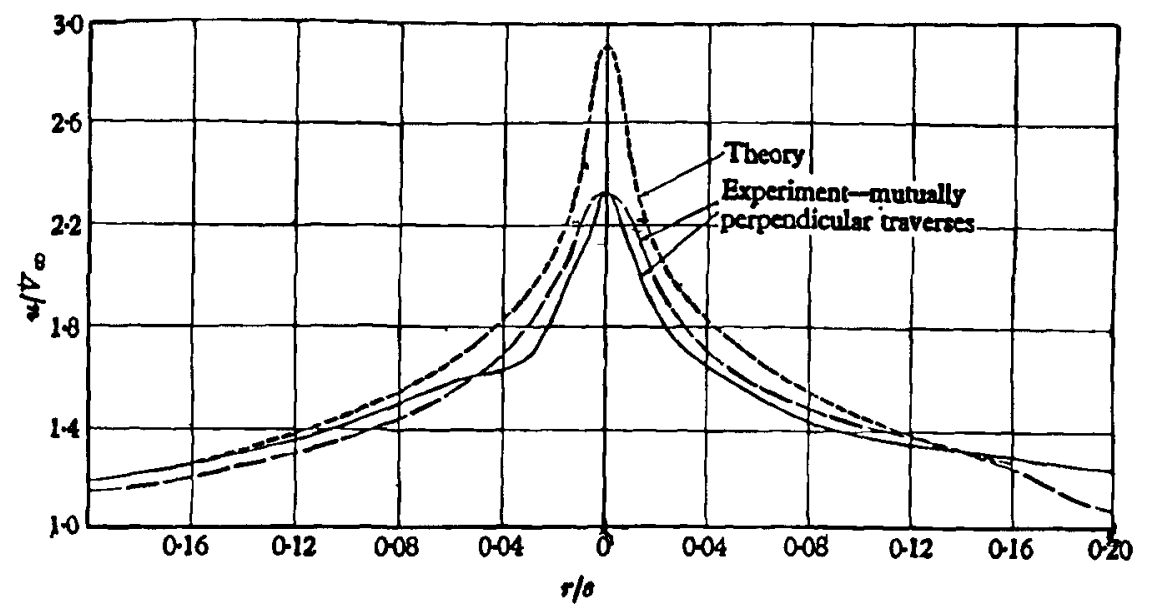

Figure 1.11. Theoretical and experimental axial velocity profiles ${ }^{26}$ 


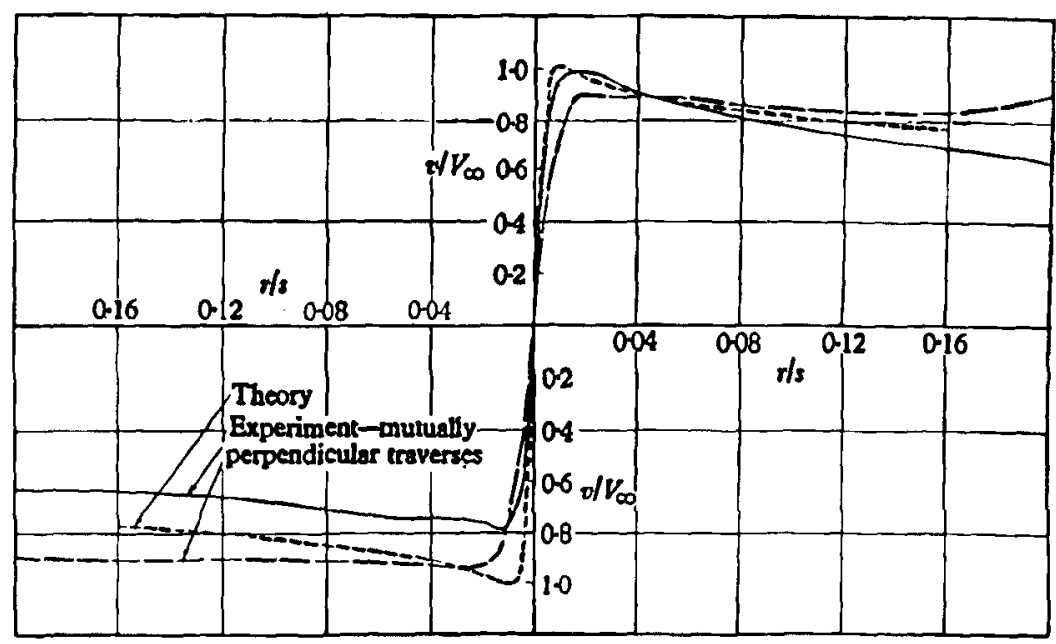

Figure 1.12. Theoretical and experimental azimuthal profiles ${ }^{26}$

As will be seen in Chapters 5 and 6, the results of the velocimetry data for the wings tested in the present experiments are quantitatively quite different, though the $65^{\circ}$ wing follows the general trends of Figure 1.11.

The inviscid analysis of Moore and Pullin ${ }^{28}$ considers a nonslender wing in the first sense, but not in the second; the wing is semi-infinite but of possibly low sweep. They find that the primary LEV trajectory is a function of sweep, but is invariant with angle of attack. Interestingly, a numerically realizable solution with coherent, rolled-up leading edge vortices was found for sweep angles down to $0.5^{\circ}$, as shown in the sketches of Figure 1.13 (adapted from ${ }^{28}$ ). 


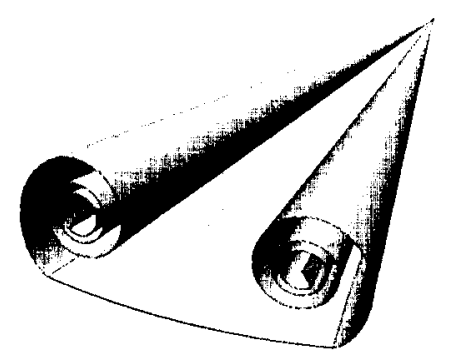

$\Lambda=75^{\circ}$

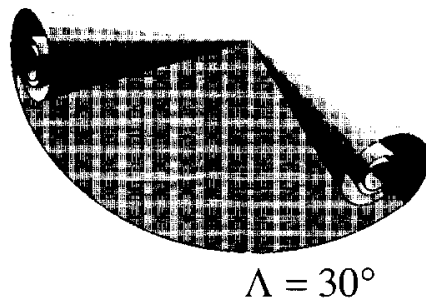

Figure 1.13. Conical vortex sheets from theoretical solution ${ }^{28}$

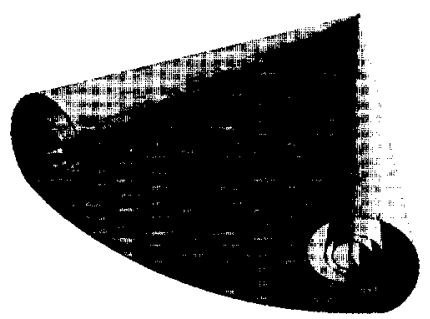

$\Lambda=60^{\circ}$

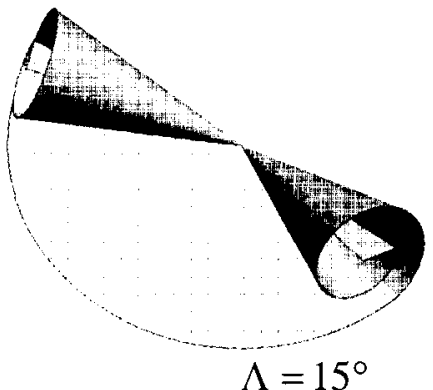

$\Lambda=15^{\circ}$

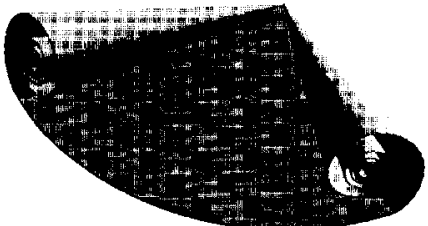

$\Lambda=45^{\circ}$

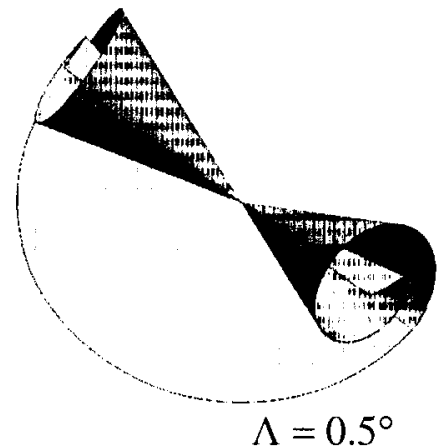

\subsubsection{Phenomenological models}

A casual perusal of the classical theoretical models of the delta wing flowfield (see for example $\mathrm{Stahl}^{29}$ ) shows rather unsatisfactory results in terms of agreement of predicted vs. experimentally observed aerodynamic coefficients. In contrast to the purely theoretical models, the model of Polhamus ${ }^{30}{ }^{31}$, based on the "leading edge suction analogy," has been very successful in its agreement with experiment. Here the lift of the wing is separated into the "potential" and "vortical" contributions. The former is due to nominally attached potential flow over the wing, and the circulation of that flow as required by the trailing edge Kutta condition. This is conceptually akin to the lifting line theory of classical wings (see e.g., Kuethe and $\mathrm{Chow}^{32}$ ). The latter is ascribed to the underpressure due the LEVs. More precisely, the leading edge "suction" of classical airfoils, responsible for turning the lift vector away from the normal to the airfoil chord 
line to the normal to the free stream velocity direction, is considered to be absent for the slender delta wing with sharp leading edge. Instead, this suction is rotated to the normal to the wing planform, resulting in a lift component. One then speaks of a leading-edge Kutta condition, wherein the flow separating at the leading edge separates in a particular way, and is assumed to reattach elsewhere along the wing leeward surface. Comparison with the schematic of Figure 1.1 suggests that this is reasonable. However, this model also breaks down for sufficiently nonslender wings, as the leading edge Kutta condition is intuitively expected to fail with decreasing sweep. In the present work, an effort is made to consider evidence of this in terms of the velocity field in crossflow planar cuts, for a wing of relatively low sweep.

\subsection{Leading edge vortex stability vs. existence}

In an effort to predict the high angle of attack behavior of LEVs, various models for predicting vortex breakdown location have been proposed. Gursul ${ }^{33}$, for example, considers several experimental data sets for $65^{\circ}, 70^{\circ}, 75^{\circ}$, and $80^{\circ}$ wings, and compares VB criteria based on conditions of the LEV (such as peak circulation and swirl angle the ratio of peak azimuthal to peak axial velocities) and effective angle of attack at the leading edge (thus, the combined effects of sweep and AOA). Generally, these prediction criteria are tuned for slender wings. As such, they presuppose the existence of a LEV, and then ask, "When will it break down?" That is precisely the question to ask for slender wings.

It is well known that as one reduces the LE sweep, LEV-type structures become less prominent and eventually cease completely. For a given sweep angle, there are 
lower and upper bounds of AOA between which stable LEVs are expected. The present experiments have found stable LEVs for very low $\mathrm{AOA}$, at least down to $5^{\circ}$, for a $65^{\circ}$ wing and for a $50^{\circ}$ wing. However, there must exist a LE sweep angle for which LEVs are no longer sustainable for any angle of attack. So for less slender wings, a more critical question is, "How does one explain the fact that a coherent LEV is not formed?" The distinction is that of eventual destruction of the LEV due to vortex breakdown vs. its inability to have formed in the first place.

Wedemeyer ${ }^{34}$ considered precisely this question, by applying Ludwieg's stability criterion $^{35}$ for rotating flows to the LEV core, and using Hall's asymptotic model of the core velocity profiles. This resulted in a stability (Figure 1.14) diagram for whether a LEV can exist. In the figure, $K$ (later referred to as $K_{W}$ ) is the "profile parameter," which is related to the swirl angle, which in turn is related to sweep angle and angle of attack. The $\mathrm{x}$ - and $\mathrm{y}$-coordinate axes in the figure are nondimensionalized gradients in circumferential and axial velocity, respectively. Beyond a certain threshold, stable LEVs are conjectured to no longer be possible.

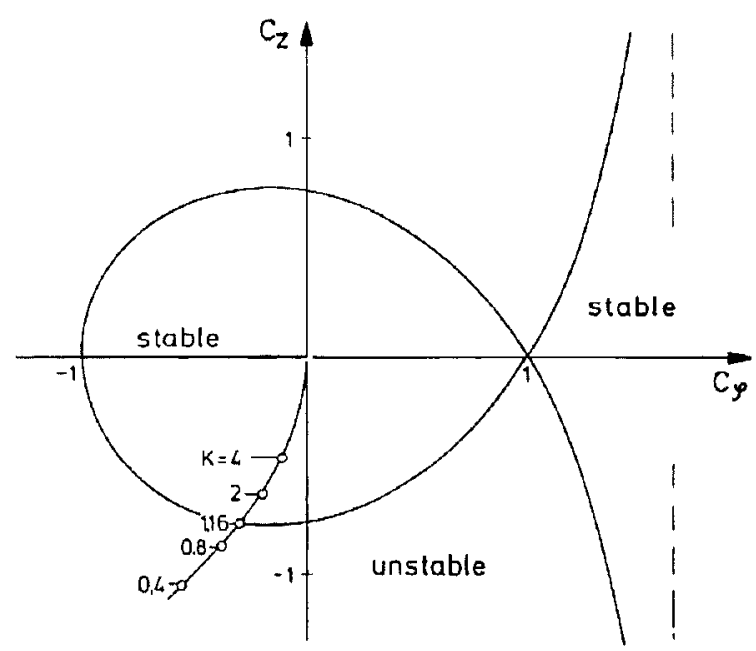

Figure 1.14. LEV stability diagram (from Wedemeyer ${ }^{34}$ ) 
The conclusions of this prediction will be compared to experimental measurements, for both the "relatively slender" baseline model, and the nonslender model.

The present study focuses on relatively low angles of attack, i.e., those around $20^{\circ}$ and below. At high angles of attack, it is firmly established that vortex breakdown limits the extent of LEVs, the alternative to LEV-dominated flow being a "blanket" separated flow identifiable with one of the top three flow regimes in the caption to Figure 1.9. But at the lower angles, the problem is more involved.

\subsection{Applications of PIV to delta wing flows}

The past decade has seen considerable activity in the use of PIV-based methods in delta wing research. Most investigations were based on two-dimensional implementations of PIV.

Cipolla $^{36}$ used a version of 2D PIV to consider "instantaneous velocity fields, contours of constant vorticity, and sectional streamline patterns" in crossflow planes and planes coincident with the LEV core axis. She also considered the vorticity due to crossflow plane in-plane velocity (that is, the streamwise component of vorticity), for the LEV feeding sheet and the secondary vortices. Such measurements illustrated very well the distinction between streamise stations upstream and downstream of breakdown.

Lang and Limberg ${ }^{37}$ took images with photographic PIV in vertical planes aligned with the streamise direction (thus, cutting the wing at different stations along the span, rather than the chord). These were then assembled by a scheme based on the continuity equation, to yield quasi-three-dimensional data. 
Shih and Ding ${ }^{38}$ also applied PIV in crossflow planes to delta wing flows, constructing contours of axial vorticity in crossflow planes. "Islands" of vortical structures were found in the shear layer and leeward surface boundary layer, with some evidence of secondary LEVs as well.

Romano $^{39}$ conducted PIV and LDV measurements in the wake of a $70^{\circ}$ delta wing in a water tunnel.

Shah et al. ${ }^{40}$ used a 2-D version of digital PIV (similar to the one used in the present investigation) in crossflow planes in the near wake of a half-delta wing of $60^{\circ}$ sweep, at $30^{\circ}$ angle of attack (thus, in a regime where VB is essentially at the wing apex), to measure turbulent mixing and axial vorticity transport.

Spedding et al. ${ }^{41}$ used similar methods to study the circulation over the leeward side of a $70^{\circ}$ delta wing with and without leading edge flaps, at $10^{\circ}$ angle of attack.

Towfighi and Rockwell ${ }^{15}$ used a photographic version of PIV to image the breakdown structure of a delta wing LEV.

Traub et al. ${ }^{42}$ used 2-D digital PIV and hot wire measurements to study postbreakdown time-dependent phenomena for a delta wing of $75^{\circ}$ sweep.

It is interesting to note that all of these investigations were concerned with wings of $60^{\circ}-75^{\circ}$ leading edge sweep.

\subsection{Experiments with lower sweep delta wings}

From about the 1950's onward, experimental results suggested that the stable counter-rotating leading edge vortex pair, so characteristic of slender delta wings, is very weak or entirely absent by for sweep angles of 45 degrees or less. 
Lambourne and Pusey ${ }^{43}$ tested a sharp-edged plate with rounded tip - effectively, a rectangular wing half-model - inclined at various angles of sweepback and attack. For zero sweep, they observe the typical leading-edge separation bubble for a rectangular wing, for angles of attack as low as $2^{\circ}$. At $20^{\circ}$ or even $10^{\circ}$ angle of attack, they observe a "bluff body" separation emanating from the leading edge, and developing downstream. For wings of $30^{\circ}$ and greater sweep, at very low angles of attack, they note the presence of outboard flow adjacent to the windward surface, with the strength of outboard flow increasing with increasing sweep. By $6^{\circ}$ angle of attack, tracer particles introduced upstream of the leading edge have "looped" trajectories, perhaps indicative of nascent LEVs. For wings of $60^{\circ}$ sweep, they find yet stronger outboard flow, this time "almost aligned with the leading edge." For angles of attack in the $10^{\circ}-20^{\circ}$ range, they note that the wing of $60^{\circ}$ sweep has a concentrated vortex core, which in modern language would be recognized as an LEV. But at $45^{\circ}$ sweep and to some extent even at $30^{\circ}$ sweep, they find evidence of similar structures, though much greater in diameter (more diffuse) and nominally more turbulent. Thus, their experiments indicate the presence of leading edge vortex motion for sweep angles well short of "slender wings," although this motion is not the well-concentrated motion of classical LEVs.

Honken and Andreopoulos ${ }^{44}$ used a "triple orthogonal hot wire probe" to measure the flowfield about a $45^{\circ}$ wing at $15^{\circ}$ angle of attack. At a crossflow plane stationed at $\mathrm{x} / \mathrm{c}=0.85$, they report the presence of a broad LEV with peak core axial flow speed of $1.3 U_{\infty}$, and discrete longitudinal vortical structures around the perimeter of the LEV feeding sheet. Comparison with the available literature suggests that this flow is almost certainly post-breakdown. However, the moderate ratio core axial to free stream 
velocities, in contrast with the much larger ratio reported for more slender wings, is consistent with the data obtained in the present study for flow upstream of breakdown for moderate angles of attack.

Miau et al. ${ }^{45}$ conducted a series of water tunnel flow visualization experiments for $50^{\circ}$ wings with various leeward and windward bevel angles. They found that round, square, to windward-beveled leading edges result in what appears to be organized LEV structures at angles of attack of 10 and 15 degrees, but leeward side bevels and double (symmetrical) bevels apparently in general suppress LEV formation. For a $25^{\circ}$ windward bevel at $10^{\circ}$ angle of attack, they find a classical LEV-type flow pattern in the crossflow plane. But for a $25^{\circ}$ leeward bevel under otherwise identical conditions, they find a flow pattern somewhat akin to the leading edge separation bubble of a 2-D airfoil with sharp leading edge.

The leeward bevels effectively have a smaller included angle for the flow to turn in going around the leading edges, evidently setting up a smaller pressure gradient for the flow to overcome. That conceivably explains the flatter, much smaller vortical structures of the leeward beveled wings. At higher angles of attack, in marginal cases the pressure gradient is large enough to return to classical delta wings LEVs. But in the most pathological cases, the pressure gradient is evidently never strong enough.

Zohar and $\mathrm{Er}^{-\mathrm{El}^{46}}$ considered the relative contribution of LEVs to the normal force for delta wings of $55^{\circ}-75^{\circ} \mathrm{LE}$ sweep, by measuring leeward surface pressure distributions. As expected from intuition, and from the predictions of models such as that of Polhamus ${ }^{30}$, the LEVs were found to have progressively smaller contributions to the total normal force as aspect ratio increases. The implication of aeronautical interest is 
that VB is less devastating for nonslender than for slender wings. The implication for more abstract interest is that the breakdown process itself evidently does not completely annihilate coherent flow separation about such wings.

\subsection{Prospects and conjectures}

The strongly three-dimensional nature of the nonslender delta wing warrants the implementation of a three-dimensional diagnostic technique. Stereoscopic particle image velocimetry has been adapted for this purpose. The velocity data allows one to make statements regarding the following:

- verification of existence of LEV-type structures, and of the location of LEV cores

- verification of the existence and location of secondary vortices

- identification of regions of reversed flow, e.g., just inboard of the leading edges

- identification of planar projections of streamlines

- flow statistics (in a baseline study, the mean and root mean square velocities), and hence information about the degree of bulk flow unsteadiness

- LEV circulation

- distribution of axial [and in a limited sense, all three components of] vorticity.

The 3-component planar nature of stereo PIV measurements of the delta wing flowfield in crossplane cuts can be qualitatively compared to the "tuft grid" measurements of the 50's and 60's (Bird $\left.{ }^{47}, 1969\right)$. There, the wing pierced an array of tufts, and a downstream camera photographed the deflection of the tufts. While quaint by modern standards, this technique does produce a velocity "vector" field. The main drawback of the technique - uncertainty whether the tuft motion captures the local fluid motion - is eerily reminiscent of the principal uncertainty of piv - whether the imaged particle motion captures the local fluid motion.

The present work proposes that the classical vortex breakdown issues have to be modified for sufficiently nonslender wings. The main issue is not the kinetics of the 
vortex itself, but the vorticity balance set up by the flowfield about the leading edges that is, vorticity generation (the separating shear layer and the secondary vortices) and vorticity convection away from the point of generation. Lee and $\mathrm{Ho}^{48}$ remark that a stable LEV is possible only when the vorticity generation is balanced by convection along the core axis. And "on a 2-D airfoil, separation from the leading edges does not lead to a stationary vortex, because there is no intrinsic convection to remove vorticity from the core." Strong convection along the core axis can intuitively be identified by the high core axial velocity, such as that described in Figure 1.11. As will be seen subsequently, such a clear velocity peak was not observed for the delta wing of $50^{\circ}$ leading edge sweep, under the conditions of the present experiment, regardless of angle of attack or location of vortex breakdown region.

How a wing generates lift depends on how it generates vorticity in the flowfield. Performance of the wing depends on whether the mechanism for vorticity generation is itself stable and predictable. As a first step toward that assessment, the present work considers clues in the (3-component) velocity distribution in crossflow planar cuts, and the behavior of quantities derived from the velocity field.

In the following, Chapter 2 discusses the stereo digital particle imaging velocimetry technique, and its present implementation. Chapter 3 describes the experimental apparatus, including the water tunnel constructed for these experiments. Flow visualization data, obtained by dye injection near the wing apex, are presented in Chapter 4, while the SPIV data are given in Chapter 5. Chapter 6 considers some of the consequences of the flow visualization and SPIV results. We conclude with Chapter 7. 


\section{Chapter 2 Rotational Stereo Digital PIV in a Water Tunnel}

In this chapter, the rotational version of stereoscopic digital particle image velocimetry (subsequently referred to as SPIV) is proposed as a diagnostic tool for studying delta wing flows, and is described in its present implementation. Issues of image post-processing and data reduction are considered. The adaptation of SDPIV to the water tunnel environment is described, and some validation cases are considered. The operations of "classical" DPIV on which the stereo version is based, and the various optical and signal processing considerations of the former, are deferred to Chapter 3.

\subsection{Velocimetry techniques for delta wing flows}

Accurate determination of the radial, axial, and azimuthal components of velocity inside the cores of vortices generated by leading edge separation over sharp edged delta wings at high angle of attack has been a problem of interest for quite some time. Ideally, one would take a fully three-dimensional, instantaneous measurement of all three velocity components. But this is of course no more than an amusing fiction. Existing velocimetry methods include on the one hand pointwise diagnostic tools, such as Pitot and multi-hole probes, hot wire probes, and laser-Doppler velocimetry, and on the other hand, "field" diagnostics, such as particle image velocimetry and its various extensions. While a rigorous review of the relative merits of these techniques is rather beyond the scope of the present work, a cursory discussion is in order.

Inspection of Figure 1.11 and Figure 1.12 shows that even for a slender delta wing, large deviations in velocity relative to the free stream can not be confined to any given measurement plane. Flow about a delta wing can legitimately be called fully threedimensional, and this fact of course affects the choice of experimental method in 
measuring the velocity field. 3-D considerations are especially significant in the case where the wing aspect ratio is not so low that the slender body theory, with its quasi-2D assumptions, can be invoked to justify $2-\mathrm{D}$ measurements.

Techniques that measure velocity at a point or a small volume would require huge test matrices to walk about a coordinate system around the region of interest, while still covering a fully $3-\mathrm{D}$ domain. This is time consuming and invites interpolation and discretization errors. In addition, such an approach is limited to capturing time-averaged data, unless the test matrix is traversed on a time scale greatly smaller than the flow time scale of interest. Instantaneous details, such as the possible meandering of the primary vortex core or intermittent reversal of the secondary vortex trajectory would only be recorded as "smears." The problem would be especially acute for nonstationary flow features, such as those documented in Section 4.4.3 below.

Also, the flow is in certain portions sensitive to minor disturbances from intrusive probes. Payne et al. ${ }^{49}$ compared the results of LDV and a seven-hole pressure probe in the LEV core, and concluded that data from the two techniques was in reasonable agreement when the LEV was not subject to breakdown. However, probe interference did affect breakdown itself. Clearly, a "sensitive" flow condition would be more susceptible contamination from an intrusive instrument. The $50^{\circ}$ wing, with its relatively weak LEVs, would be especially susceptible to possible probe interference. This is a strong argument in favor of nonintrusive techniques. These can be also be classified as discrete-point techniques (such as LDV) and field techniques, such as PIV. Combining the considerations of non-intrusive diagnostics and desirability of field techniques, PIV becomes a natural choice. 
But to address three-dimensionality, extensions beyond the usual 2-D PIV are desirable.

\subsection{Applications of PIV}

\subsubsection{Planar PIV}

At its foundation, PIV is a planar technique, in which one measures the twocomponent velocity field restricted to a two-component domain, the planar interrogation region. But even if one were to be interested purely in the in-plane flow components, the out-of-plane flow still can not be ignored, since it can have a serious adverse effect on the quality of in-plane data, due to errors introduced from loss of correlations between PIV image pairs. Particles photographed in the light sheet at one instant of time do not all appear in the next instant, since some have convected out of the light sheet. Still other particles, which were not in the light sheet at the first instant of time, may have convected into the light sheet at the next instant, and would then be recorded in the second frame of an image pair, but not in the first. In the event that the out-of-plane motion is uniform, the problem is solved by advancing the light sheet at the time that the second frame is captured. Thus, the light sheet pair is no longer coincident, but separated in space by a displacement dependent on the ratio of in-plane to out-of-plane velocities. Alternatively, the light sheets can be kept coincident, but made very thick. Here the problems include loss of available laser light intensity due to lack of concentration in a thick sheet, and the exacerbation of the 2-D inaccuracies due to 3-D optical effects.

In addition, out-of-plane motion introduces parallax error in the in-plane data, wherein motion purely normal to the light sheet has an apparent in-plane signature.

\subsubsection{Some extensions of planar PIV}


Two "3 component planar" versions of PIV are widely mentioned in the literature - the so-called "dual plane" PIV, and stereoscopic PIV.

Dual plane PIV uses a triplet of light sheets, with suitable spacing between the sheets. This allows for two particle image pairs - that of sheets $1-2$, and sheets $2-3$. The magnitude of the correlation peaks in the first vs. the second pair are compared, and from this one obtains a measure of the out-of plane component ${ }^{50}$. This methodology is a departure from the usual case, where only correlation peak locations (but generally not magnitudes) are considered. When the peak magnitude is important, the level of accuracy of the experimental setup is placed under considerably higher requirement, severely impacting the robustness of the technique. Indeed, dual-plane PIV was initially attempted in the present experiments, but soon abandoned.

Stereo PIV, on the other hand, is a direct extension of regular PIV. Two cameras at two different locations, subsequently referred to as the left and right, are focused on the same region of interest in the flowfield. Various arrangements are possible (see for example Prasad ${ }^{51}$ ) but the common idea is to compare geometric differences in the inplane particle displacement field as viewed by the two cameras, obtaining out-of-plane information from differences in between the two images. The result is a full threecomponent data set, with provision for comparable accuracy in all three directions. The data are of course still restricted to planar slices of the flowfield.

Stereo PIV has the important advantage that instead of constructing an entirely new image interrogation scheme, the usual two-dimensional PIV analysis is retained. The three-dimensional data are obtained by means of simple trigonometric relations. Further image processing on the left and right images is typically necessary, but it is 
seamlessly appended to the two-dimensional processing. Using the very same calculations used to combine 2D data to obtain 3D data, stereo PIV can also improve the accuracy of 2D data by taking into account the effect of particle motion normal to the light sheet (though in fact in its present implementation, this particular matter contains some unresolved issues, as discussed in Chapter 3 and Appendix B). This is typically ignored by regular PIV, and can lead to large perspective viewing errors, especially when the light sheets are thick.

\subsection{Stereo PIV applied to the present experiment}

A synopsis of SPIV variants is given below. Image processing concerns are described next. Finally, the physical implementation is described. Since the present work is one of technique implementation rather than technique development, emphasis is placed on the former. Particular attention is given to those difficulties in implementing SPIV due to the change in index of refraction in going from air to water.

\subsubsection{Camera orientations in SPIV}

For implementing stereo PIV, two schemes of camera orientation have found common use in the literature: "translation SPIV," and "angular (or rotational) SPIV." Both are based on adaptations of optical techniques used in classical large format still photography, where the camera and object planes are not necessarily collocated on the same optical axis. Broadly speaking, the former involves shifting the camera film plane (or in the present case, the $\mathrm{CCD}$ plane) relative to the object plane, and the latter involves rotating the film plane. The rotational scheme, while requiring more intensive image post-processing, has been shown to be more robust (see e.g., ${ }^{54}$ ). Specifically, it allows 
for greater field of view for the same optical conditions, because the translational scheme generally does not have full-frame left and right image overlap. Also, the rotational scheme offers better control over accuracy in the out-of-plane velocity component ${ }^{85}$. Common to both techniques is the attempt to control depth of field and perspective when viewing three-dimensional objects.

In the present study, the choice of setup was motivated more by practical considerations, rather than an attempt at achieving maximal accuracy. As such, a translational arrangement would be awkward to adapt to a water tunnel, with the lenses and cameras presumably outside the test section. The rotational arrangement can be implemented with a simple mirror system, as described in Section 2.6.3.

\subsubsection{The Scheimpflug criterion}

Placement of the cameras at angles other than normal to the interrogation region results in a condition where one side of the interrogation region is further away from the lens than is the opposite side. This introduces a problem with depth of field; that is, since the interrogation region is no longer contained in a plane normal to the lens axis, the entire image is never in focus for any lens focal setting. The problem is especially bad for larger magnification ratios, where depth of field is less. Depth of field can be increased by reducing the lens aperture, but this is of limited use in practice, since the intensity of the optical signal of the tracker particles is rarely bright enough to allow for the higher aperture settings of a typical $35 \mathrm{~mm}$ photographic lens.

Provided that the manipulations are not so great that the lens can no longer coherently image the portion of interest of the interrogation plane onto the film plane, one can use tilt of the film plane with respect to the lens plane to increase the effective depth 
of field, by realigning the focal plane with respect to the interrogation plane. This is done by aligning the subject plane, lens plane, and camera plane according to the "Scheimpflug condition" (Figure 2.2). In this arrangement, the three respective planes intersect at a common line, and the angle $\varphi$ is specified by the lens-to-camera distance. This distance is invariant with tilt, and is a function of the lens design. For lenses used in $35 \mathrm{~mm}$ photography, this distance is about $50 \mathrm{~mm}$, measured from the lens mounting face to the ccd plane.

After focusing the lens such that the center of the image is in focus, the film plane is rotated with respect to the lens, until the entire image appears in focus. Meanwhile, the lens is held fixed. Doing so requires the construction of a camera apparatus that mimics the rear-tilt large format photographic camera. The apparatus used in the present experiment uses a pair of Nikon-mount $35 \mathrm{~mm}$ photographic lenses and ccd camera heads, in this case of $640 \times 480$ pixel density, all fitted to a pair of aluminum housings which allow rotation of the camera heads. A photograph of one camera/lens unit is shown in Figure 2.1, with the cover removed.

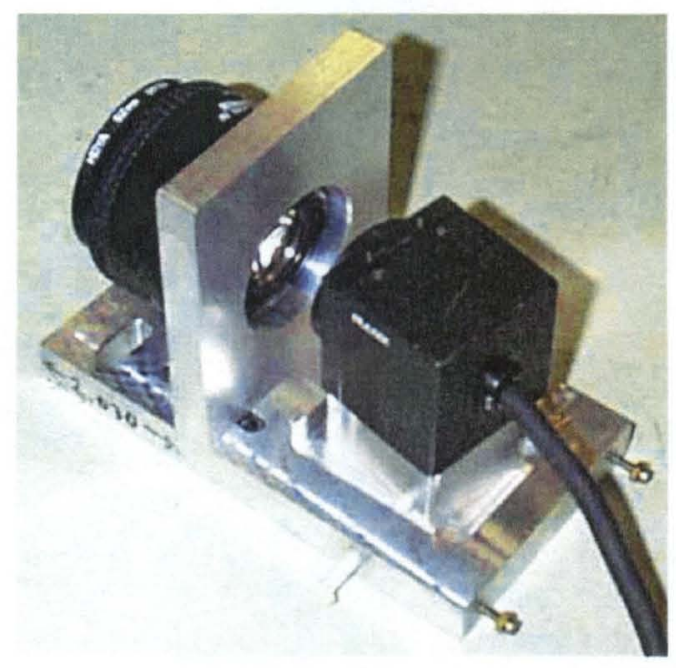

Figure 2.1. Photograph of stereo PIV camera and lens arrangement 


\subsubsection{Rotational camera arrangement}

A typical arrangement is shown in Figure 2.2, with the lenses and cameras represented schematically.

Besides the various practical considerations of how to mount and orient the cameras in the laboratory setup, one has to consider such parameters as the "optimum" angle between the cameras and the viewing plane, in terms of minimizing in-plane and out-of-plane measurement errors. Lawson and $\mathrm{Wu}^{85}$ report that the ratio of out-of-plane to in-plane error decreases rapidly as the camera included angle is increased from zero, but then levels off for larger angles. For a $45^{\circ}$ viewing angle, as shown in the figure, the in-plane and out-of-plane errors are comparable. The absolute measurement error is limited by the 2-D PIV interrogation and processing resolution.

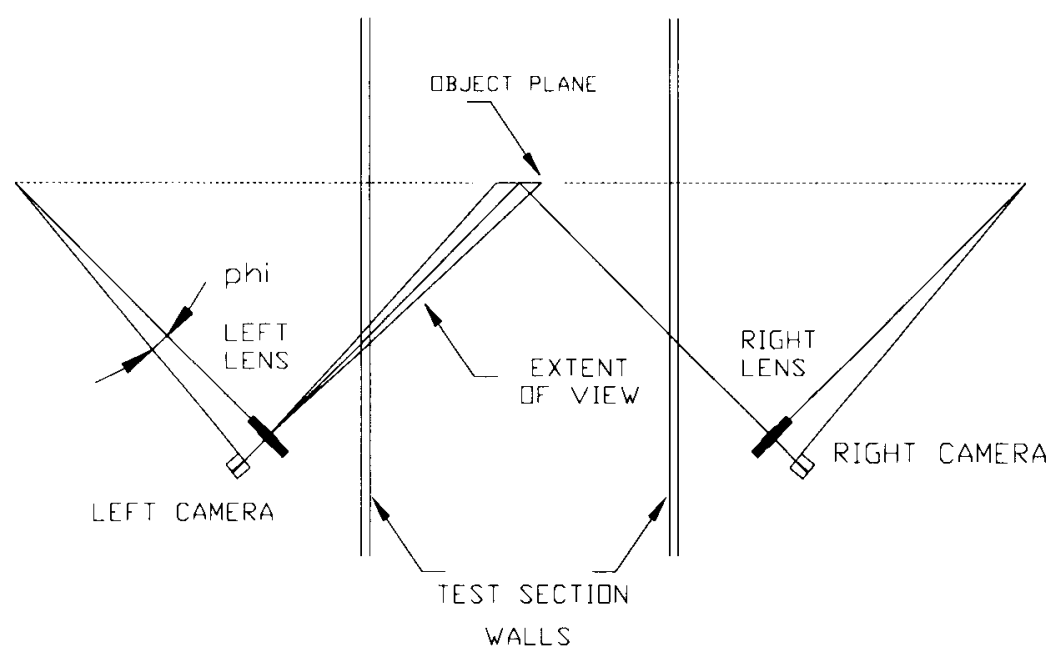

Figure 2.2. Camera and lens arrangements with Scheimpflug criterion, top view

\subsection{SPIV "triangulation"}


The "stereo" part of SPIV involves the merging or "triangulation" of the left and right data, to form an image with "depth perception." The basic methodology is described in this section. Refinements are mentioned further below.

In Figure 2.3, the left and right cameras are shown facing the interrogation plane at some orientation in space, viewing a typical 3-D velocity vector. Each camera records images according to the standard methodology of 2-D DPIV, obtaining a pair of 2-D velocity vector fields. These are then combined to form the three components of velocity in a planar slice, written as $\vec{u}=(u(x, y), v(x, y), w(x, y))$, by trigonometric manipulations motivated by Figure 2.3 and Figure 2.4 .

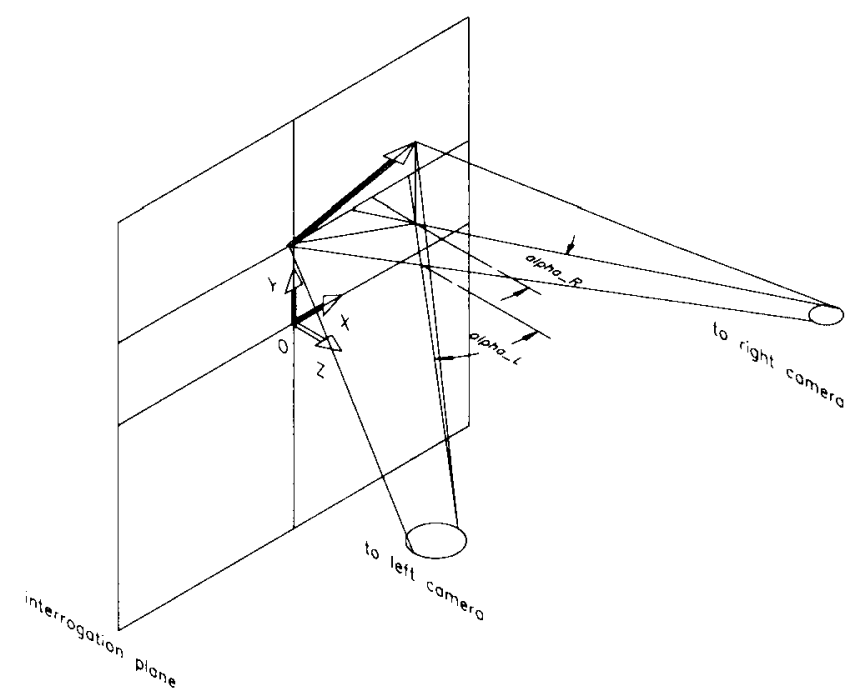

\section{Figure 2.3. Stereoscopic representation of displacement vector}

Camera locations with respect to the interrogation plane are described by four angles. Consider the coordinate system $(X, Y, Z)$ centered about the origin of the interrogation plane, $O$. The left camera is inclined from the normal at $O$ by the angles $\alpha_{L}$ (in the $X Z$ plane, the "horizontal" plane) and $\beta_{L}$ (YZ plane, the "vertical" plane); the respective angles are $\alpha_{R}$ and $\beta_{R}$ for the right camera. Note that this coordinate system is 
inconsistent with the canonical usage in aerodynamics. The present convention is maintained in the delta wing velocity vector fields discussed in subsequent chapters.

In Figure 2.4, the projection onto the $\mathrm{XZ}$ plane is shown. The candidate velocity vector has components $d z$ (the "out-of-plane" component) and $d x$ (the "in-plane horizontal component; the in-plane vertical component is suppressed in the planar projection). These two components are viewed differently by the left and right cameras.

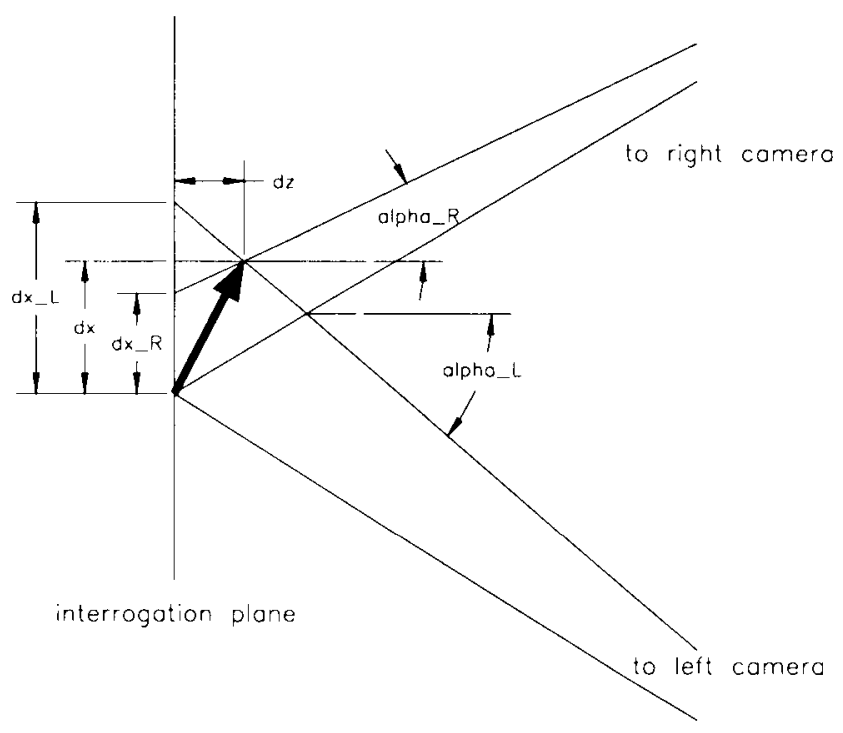

\section{Figure 2.4. Stereoscopic representation of displacement vector, top view}

In the general case, the nonzero camera inclination can be set up in both the horizontal and vertical orientations. This results in the need to specify all four viewing angles discussed above. To obtain the 3-component velocity field, we have the following inputs:

left camera planar data, $\quad u_{L}, v_{L}$

right camera planar data, $\quad u_{R}, v_{R}$

camera viewing angles: $\quad \alpha_{L}, \alpha_{R}, \beta_{L}, \beta_{R}$ 
From the schematic representations of how a typical displacement vector is rendered in the left and right camera views, the following formulas for the true vector components are obtained:

$\mathrm{x}$-component (in-plane, horizontal):

$$
u=\frac{u_{L} \tan \alpha_{R}+u_{R} \tan \alpha_{L}}{\tan \alpha_{L}+\tan \alpha_{R}}
$$

y-component (in-plane, vertical):

$$
v=\frac{v_{L} \tan \beta_{R}-v_{R} \tan \beta_{L}}{\tan \beta_{R}-\tan \beta_{L}}
$$

$$
\text { and } \quad \beta_{L}, \beta_{R}=0 \rightarrow v=\frac{v_{L}+v_{R}}{2}
$$

The result for the y-component follows from a similar argument, by appealing to the $Y Z$ planar projection. In the limit of zero $\beta$, the $y$-component reduces to an arithmetic average:

z-component (the out-of-plane):

$$
w=\frac{u_{L}-u_{R}}{\tan \alpha_{L}+\tan \alpha_{R}}
$$

For a more precise evaluation of the y-component (in the sense of stable numerical calculation by computer) in the case of small but nonzero $\beta$, we use information about the Z-component:

revised y-component:

$$
v=\frac{v_{L}+v_{R}}{2}+\frac{w}{2}\left(\tan \beta_{L}+\tan \beta_{R}\right)
$$


These formulas are accurate when the object-to-camera distance is much larger than the size of the object (the interrogation region). A correction is advisable in the case where this is not true, i.e., where betas and alphas vary significantly over the interrogation region. Otherwise, the computed velocity field will have a geometric bias error away from the image center.

\subsection{Image processing considerations}

\subsubsection{Dewarping: motivations}

While tilting the camera solves the problem of depth of field, it still does not produce images that are immediately useable. Since the cameras are positioned away from the normal to the interrogation region in the horizontal plane, the left side of the interrogation region is closer to the left camera lens axis than is the right side, and vice versa. Perspective error results. Fortunately, the image is stored in digital format, and therefore perspective and other errors can be removed by suitable digital image processing.

For purposes of clarity, we will assume in the following that the Scheimpflug arrangement is restricted to a horizontal plane, and that the cameras are aligned perpendicular to the interrogation region in the vertical plane.

As an example of the optical effects, we can consider the image of a Cartesian grid of equally spaced vertical and horizontal line segments. Distortion or "warping" is principally manifested by a convergence of receding horizontal lines and attenuation of spacing between vertical lines in the image of such a square grid (Figure 2.5). As Willert ${ }^{53}$ points out, the effective magnification factor varies in going away from the center of the interrogation region. For viewing angles very close to the normal to the 
interrogation plane, the warping is small, since it varies roughly as the sine of the viewing angle. But for viewing angles on the order of 25 degrees - such as in the present experiment - this error can not be ignored.

There are also other sources of error. For instance, one may wish to account for the typical optical distortions present in any lens arrangement (e.g., pincushion or barrel distortion).

To recover the condition in which parallel physical lines have parallel image lines, one needs to "dewarp" the images obtained by the stereo camera arrangement. The left and right images undergo different dewarpings, but as discussed below, these dewarpings are not independent of each other. Also, the left and right images are recentered with respect to one another in the post-processing. This substantially simplifies the task of aligning the cameras in the laboratory.

\subsubsection{Calibration grid}

The task of processing the raw images produced by the left and right cameras, prior to triangulating, including the dewarping procedure, will be called "calibration." As pointed out by Soloff et al. ${ }^{52}$, Willert ${ }^{53}$ and others, precision in the calibration procedure is critical to obtaining robust results with SPIV.

The basis of calibration is a simple Cartesian grid, with the center marked for alignment purposes, placed coplanar with the light sheet plane, and centered about the region of interest. The extent of the grid is equal to or slightly greater than that of the imaged region. The raw left and right grid images are mapped ${ }^{53}$ into ones with parallel lines. The mapping that accomplishes this transformation - the dewarping mapping - is 
then applied to the tracker particle images obtained by the cameras, for every frame. It is in this sense that the Cartesian grid images can be considered calibration images.

The "before" and "after" situations for a typical calibration grid (for only one side, in this case the right) are shown in Figure 2.5. Note that the "after" image has square grid cells, and is slightly smaller in physical extent, due to effects of the dewarping mapping. Thus, a portion of the original image area is lost.
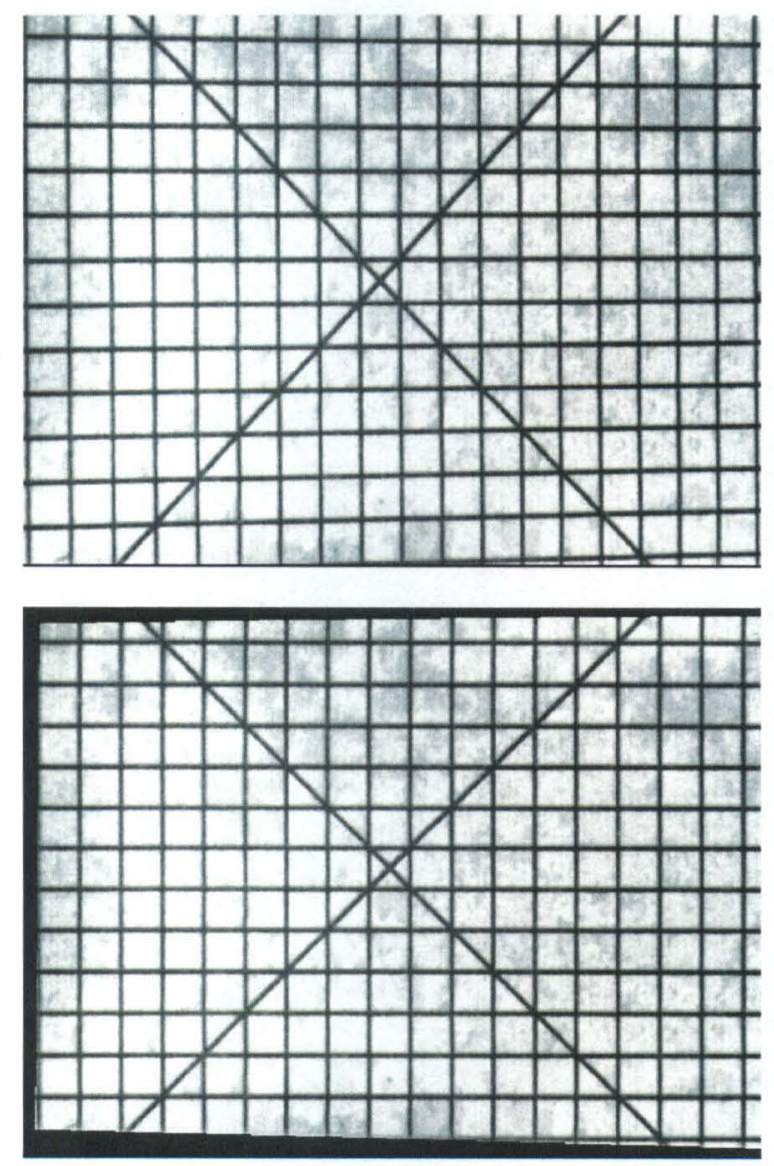

Figure 2.5. Effect of dewarping on calibration grid

\subsubsection{Dewarping: details}

The above procedure does not take into account the effects of a large viewing area, or a situation where the left and right alphas are appreciably different. The latter 
occurs, for example, for off-center viewing when the cameras zoom into one of the delta wing vortex cores. In this case, the left and right magnification factors will in general be different. Dewarping will produce properly squared images, but the scale of the left and right images will remain different, producing spurious results in the triangulation routine. A large viewing area results in substantial variation of viewing angles in going from pixel to pixel in the camera CCDs, again resulting spurious computed velocities.

The first of these problems is addressed by a refined triangulation methodology. From the original, warped grid images, one obtains the physical size of the images - the location of their left and right, and upper and lower boundaries. Then, from an accurate drawing of the grid and camera setup, the effective viewing angles $\alpha_{L}$ and $\alpha_{R}, \beta_{L}$ and $\beta_{R}$, can be calculated for each interrogation window coordinate in the left and right images. Subsequently, one applies the geometric triangulation formulas for the left and right images, also window by window. It should be mentioned that in the present implementation, the viewing angles $\beta_{L}$ and $\beta_{R}$ are zero with respect to the image center, since the cameras are in the same horizontal plane as the center of the image. However, $\beta_{L}$ and $\beta_{R}$ will vary in the vertical direction above and below the image center.

For convenience, the declared center of the image will be taken as the coordinate origin. This need not be the geometric center of the image, though it generally is. The viewing angles are calculated thus:

$$
\begin{gathered}
\alpha_{L . R}=\left|\frac{X_{\text {cam }_{L . R}}-x_{\text {image }}}{Z_{\text {cam }_{L . R}}}\right| \\
\beta_{L . R}=\frac{y_{\text {image }}-Y_{\text {ram }_{L . R}}}{Z_{\text {camm }_{L . R}}}
\end{gathered}
$$


The second problem mentioned above is resolved jointly by the dewarping code prior to running 2-D DPIV, and by a post-processing code. In this methodology, perspective information is removed by dewarping, prior to matching of the left and right images, and hence prior to 2-D DPIV calculation. In doing so, one also removes possible mismatch due to different magnification resulting from asymmetric viewing locations. The resulting 3-component velocity data are kept over a domain uncompressed by perspective distortion (that is, not effectively multiplied by the cosine of the viewing angle). Also, the 2-D DPIV methodology is left intact, performing its function on artificially squared images resembling what one would obtain from an on-axis normal view. Then, after 2-D DPIV vector fields are obtained, the post-processing code restores perspective information.

The means by which the dewarping scheme rescales the calibration grids to have square cells of uniform size is described as follows. One first considers, say, the left camera grid image. From a representative intersection near the middle of the grid, the vertical cell side length above and below is recorded. Their average value is then declared to be a characteristic cell size. This is a reasonable assumption, since the nominal beta angles (with respect to the image centerlines) are zero. This averaged vertical cell length is used to tile the entire "target image" of the grid; that is, the dewarping coefficients are obtained from a least squares fit from the raw grid image to this target image, which is composed of perfect squares. The resulting, dewarped grid image, is then "close" to this target image. The representative intersection is the fixed point of the dewarping mapping. 
Subsequently, one selects a fixed point for the other grid image. This point should be close to its counterpart in the first image; i.e., at least to within one cell side length. The right grid image then undergoes dewarping, wherein the target image is identical to that used for the left side. This ensures that the magnification of the left and right images is the same.

When the representative intersection coordinates are selected, the actual coordinates of this intersection are calculated to within subpixel accuracy. Thus, one obtains "precise" coordinates for the left and right fixed points. This allows one to translate the left and right images in the image processing, to make the respective fixed points coincident. If the dewarping routines are successful, the resulting left and right images of the calibration grid are then essentially identical. Indeed, the dewarped left and right images of a purely in-plane motion should in theory always be identical.

Once the actual particle displacement images are dewarped, image pairs from the left and the right camera separately undergo standard 2-D DPIV analysis. One then obtains "squared" left and right velocity vector data. Thus, both perspective effect (converging lines) and isometric viewing effect (image taller than it is wide) are addressed.

\subsection{Stereo PIV in a water tunnel}

Use of Stereo PIV in water involves a new set of difficulties, which arise from the unmatched indices of refraction in the water, the tunnel walls (glass), and the air outside the tunnel walls. In most cases, these effects result in apparent astigmatism, where images of nominally round particles appear to be ellipses. Focusing the camera lenses and/or adjusting the lens-to-camera angles shifts the location in the field of view where 
the astigmatism is especially bad, but does not altogether remove it. For the viewing angles used in the present investigation, a yet more serious problem results. Light does not pass from the interrogation plane to the PIV cameras outside the tunnel; instead, it is reflected back into the test section. These problems can be addressed by means of prisms placed outside the test section walls (Prasad and Jensen ${ }^{54}$ ), or by introducing mirrors inside the test section. The latter method is employed here.

\subsubsection{Corrections for multimedia optics}

In Figure 2.2, the optical path passes first through water (index of refraction, $\mathrm{n}=$ 1.333), then through glass $(n=1.52)$, and then through air $(n=1)$. This introduces the need for complicated corrections for e.g., distortions due to such multimedia optics. But a larger problem is that of total internal reflection. Indeed, an examination of Snell's law for the viewing angles involved predicts zero transmission through the test section walls to the cameras.

For small viewing angles, where astigmatism is the only problem, one can apply numerical correction methods, such as those of Soloff et al. ${ }^{52}$. But these do not solve the problem of total internal reflection. There are essentially two methods of dealing with this problem: prisms to refract the light, and mirrors to reflect it.

\subsubsection{Prisms}

Prasad and Adrian ${ }^{55}$ consider stereo PIV with a Scheimpflug arrangement in liquid flows, with the concomitant problems of mismatch in index of refraction for a liquid-air interface. Their solution is to use a thin-walled prism filled with the working liquid (water), arranged such that one wall is parallel to the lens plane. This is the natural 
solution in keeping with the non-intrusive nature of optical flow diagnostics, where the diagnostic device does not intrude into the test section.

However, this method has some drawbacks. Most important is the kinematic constraint of small subtended viewing angles; that is, the angle between principal rays going from the interrogation plane to the lenses. As pointed out by Prasad and Jensen ${ }^{54}$, the larger the subtended viewing angle, the higher the accuracy in measurement of the out-of-plane component, effectively at the expense of in-plane accuracy, and vice versa. Prasad and Jensen use an angle of about 10 degrees. For the present experiment, the baseline subtended angle is 25 degrees. For such large angles, the distortions which the prism needs to correct, and the modifications in camera placement, become quite substantial and probably intractable.

\subsubsection{Mirrors}

This leaves mirrors as a more suitable choice, at least from the optical point of view. By mounting a pair of mirrors inside the test section, the optical path in the above figure is "folded" (see below), such that light passes normal to test section walls. This removes problems of refraction. But fluid mechanically, mirrors have the severe disadvantage of disturbing the flow inside the test section, violating the principle of nonintrusive diagnostics. Mirrors should be located as far downstream from the interrogation region as possible, but that introduces constraints on magnification factor and camera subtended angle. Mirrors are also subject to deflection and vibration caused by the flow itself. 
As one might expect, for SPIV in water, the presence of the mirrors complicates matters. Specifically, the calculation of the alphas and betas requires a transformation from the "folded" Scheimpflug arrangement with the mirrors, to the "unfolded" equivalent arrangement with the mirrors removed. Measurements in the lab are taken from the folded arrangement. The unfolded quantities, necessary for the formulation given above, are calculated from some simple geometric relations. The procedure is further described in Appendix C.

With the tunnel operating, the mirrors undergo deflections and vibrations. With test section free stream speed at $80 \mathrm{~mm} / \mathrm{s}$, a mark at the centerpoint of the interrogation plane was observed to deflect approximately 1 pixel, relative to its location with the tunnel off. That deflection corresponds to $0.2 \%$ of the delta wing semi-span at the crossflow plane most commonly referred to below.

The actual laboratory arrangement is shown in Figure 2.6, which shows the top view of the water tunnel test section, the delta wing model, the mirrors inside the test section, and the lens and camera arrangement. The Scheimpflug condition is shown in a "folded" arrangement.

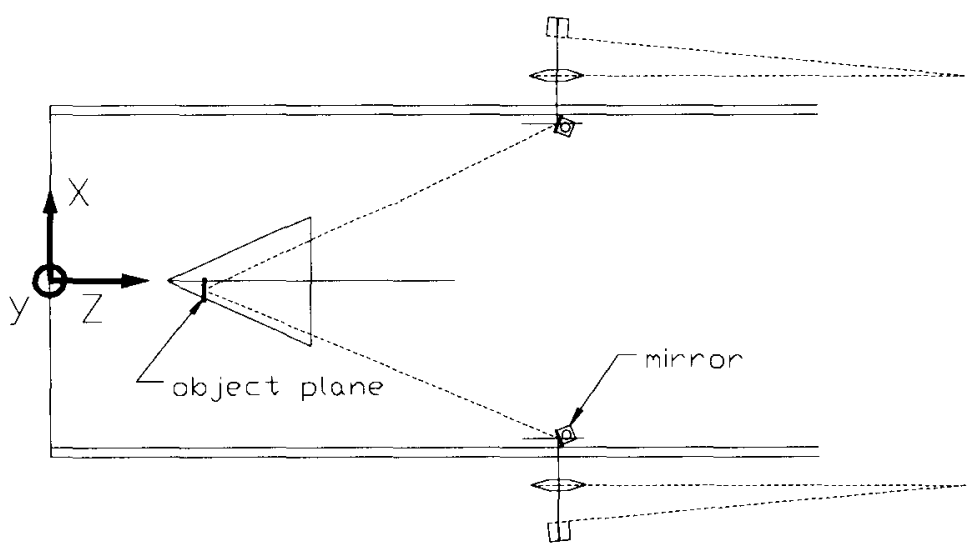




\section{$2.7 \quad$ Free stream validations}

In order to achieve some measure of confidence in the verisimilitude and robustness of the present implementation of the SPIV technique, validation experiments were performed. These consisted of comparing known frame-to-frame displacements to the SPIV measurements. Following a commonly used scheme $\left({ }^{65}\right)$, such displacements were generated by moving a target image of artificial particles. This is discussed further in Appendix B. In the following, the water tunnel free stream flow was used as a test case for SPIV measurement. This was deemed to be a reasonable choice, since the idealized free stream motion is a pure translation (the simplest possible motion), yet the fact that the motion is normal to the SPIV interrogation plane captures many of the practical difficulties of implementing the technique.

In this section, the arrangement of the lenses, cameras, mirrors, and interrogation plane is the same as that used in Chapter 5 for the delta wing velocity measurements. For consistency, physical displacement units are fractions of the trailing edge span of the delta wings, which is $180 \mathrm{~mm}$. The effective field of view is approximately $33 \mathrm{~mm}$ wide and $22 \mathrm{~mm}$ high. With respect to the water tunnel test section, the field of view has center $23 \mathrm{~mm}$ to the left of the test section midplane, and $300 \mathrm{~mm}$ above the test section floor. Flow speed, as measured independently by LDV and a separate mechanical velocimeter (a "propeller" gauge) was set at $80 \mathrm{~mm} / \mathrm{s}$. In the following, all velocities are normalized by $80 \mathrm{~mm} / \mathrm{s}$.

Discrepancy between measured and expected velocity can for convenience be split into two parts: error in the in-plane velocity components, and error in the out-ofplane component. 
Figure 2.7 shows an isometric view of the free stream velocity; this can loosely be compared to the situation in Figure B.6. The colorbar is with respect to values of out-ofplane velocity component. It is evident that at the right side of the field of view (X positive), the free stream velocity (out-of-plane component) is captured with good accuracy, but at the left side, there is an out-of-plane error of almost $6 \%$.

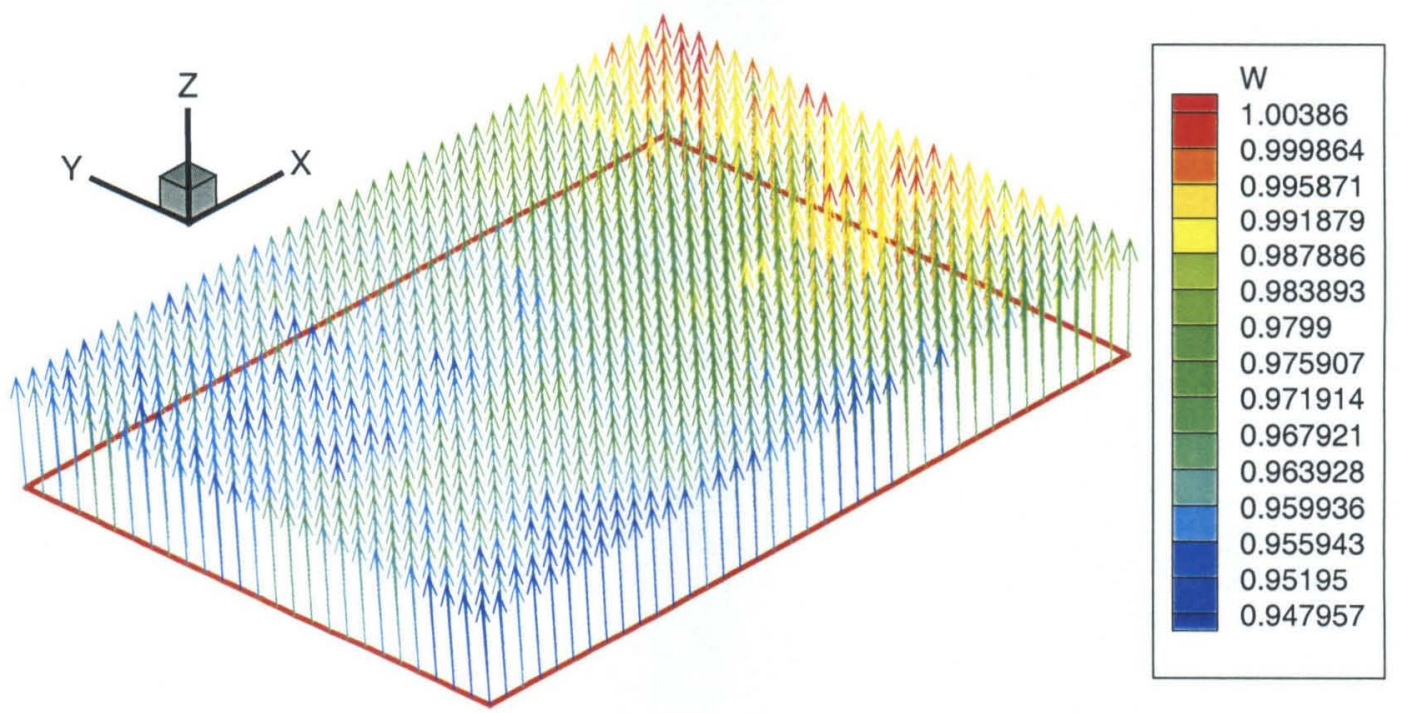

Figure 2.7. Free stream velocity, isometric view

At the image midpoint, the error in the out-of-plane component is about $2.5 \%$. It is worth noting that the highest magnitude error occurs in the flow region of least interest. The left side of the field of view lies approximately at the wing centerline, away from the leading edge vortex and its related structures.

Immediately obvious from Figure 2.7 is that the velocity vectors have an apparent in-plane component, which has the appearance of a flow away from the center of the interrogation plane. This is an in-plane error. It is best seen in a streamwise view (i.e., planar projection) of the free stream velocity, shown in Figure 2.8. Here, the colorbar 
shows the variation of in-plane error, $E$, defined for convenience as the magnitude of the in-plane velocity components:

$$
E(x, y)=\sqrt{u^{2}(x, y)+v^{2}(x, y)}
$$

E is evaluated for each PIV interrogation window.

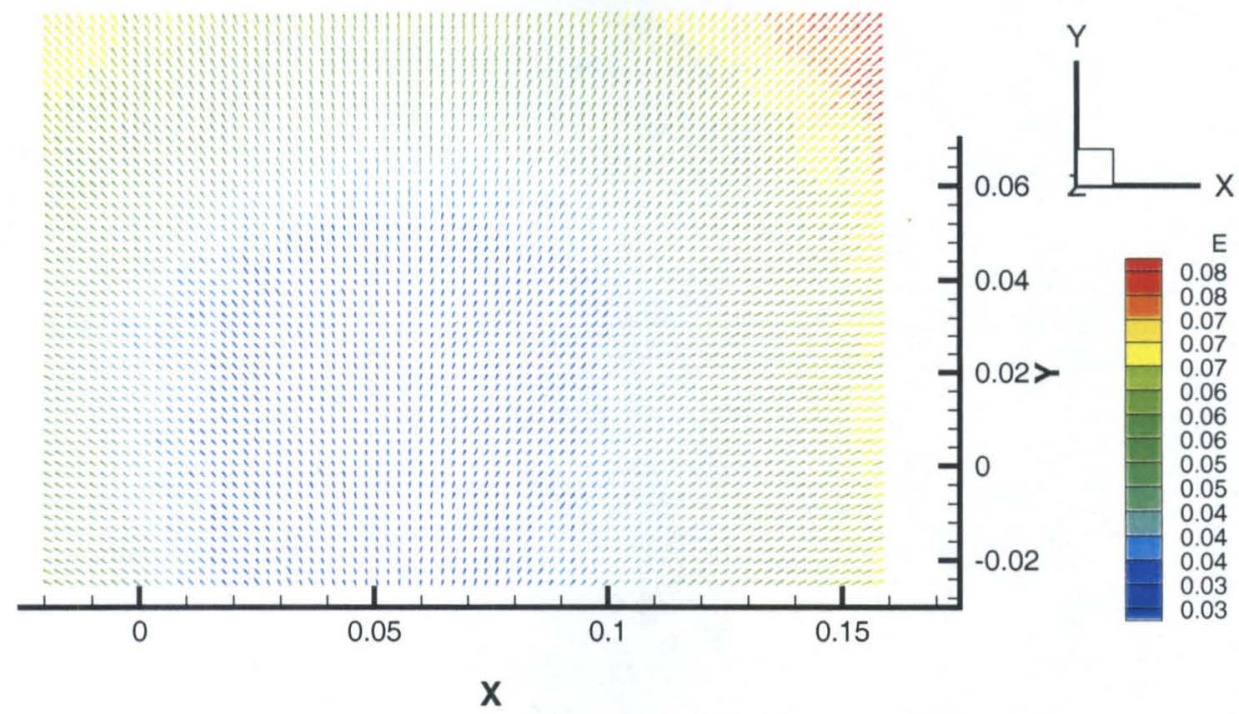

Figure 2.8. Planar projection of free stream velocity

The peak value of $E$ is on the order of $8 \%$ of the free stream velocity magnitude.

The error is systematic; the minimum error occurs at the image center, and increases in going radially away from the center. The resulting shape of the error distribution is reminiscent of parallax error, and will be denoted as such.

\subsection{Comments on parallax distortion}

While the cause of the parallax error has not been fully elucidated in the present study, perhaps the result is not surprising. As pointed out for example by Soloff et al. ${ }^{52}$, stereo PIV is an example of optical mapping from 3-D space onto a pair of 2-D surfaces (the camera ccd chips), even when the laser light sheet is thin. Constructing this 
mapping, either explicitly, as done by Soloff et al, or implicitly, as in the present work, requires calibration not only in a single plane, but in a 3-D region - or at the least, in an array of planes slicing through the light sheet. Actual location of particles would be approximated by out-of-plane interpolation between the calibration planes, much the same is in the in-plane interpolation.

Rather than attempting to handle the perspective problem with an all-inclusive 3D to twin 2-D mapping, as proposed by Soloff et al., the present work addresses perspective as an a posteriori correction. For any given data run, a validation is performed much the same as in the artificial particle speckling experiment described above. This gives information on the in-plane apparent motion as a function of out-ofplane motion, and coordinate location in the interrogation plane. This is used in data post processing. Once the 2-D PIV results are triangulated, for each interrogation window, the window centerpoint coordinate and out-of-plane component of the vector velocity are noted. These are compared to their analogues in the validation run, and the in-plane component is corrected by subtracting the appropriate "false displacement." The procedure for this candidate "solution" to parallax error is given in Appendix B.

\subsection{Summary of SDPIV procedure}

In light of the above, the present implementation of stereo DPIV can be summarized in the flowchart of Figure 2.9. 


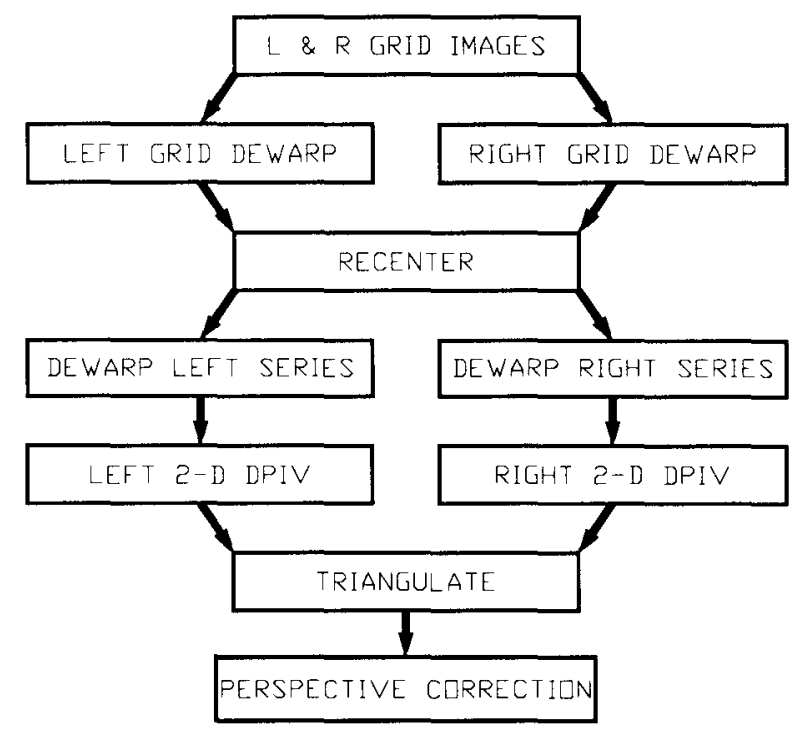

Figure 2.9. Schematic representation of computational procedure

\subsection{Application to a full-span measurement of the delta wing flow field}

We now consider a typical SDPIV result for the delta wing flow field, in crossflow planes. This establishes a baseline proof-of-concept that stereo PIV can successfully resolve the flowfield in capturing the essential features; namely, the left and right primary LEVs. However, resolution limitations of the cameras prevents one from simultaneously imaging both left and right LEVs and capturing details like the secondary LEVs. This motivates specializing to just one LEV core and the near-leading edge region on one half-span of the wing, as is done in the rest of the present work.

A sample image covering an entire cross-sectional slice (full span) of the $65^{\circ}$ wing is given below. The data are not averaged (i.e., just one image pair is used). The angle of attack is $15^{\circ}$. The measurement plane is stationed at $\mathrm{z} / \mathrm{c}=0.9$, just upstream of the trailing edge. Note that the usual choice for coordinate direction in aeronautics has been modified to conform with the SPIV optical system coordinates; thus " $\mathrm{z}$ " is the downstream direction, and " $\mathrm{x} / \mathrm{c}$ " is replaced by " $\mathrm{z} / \mathrm{c}$." 


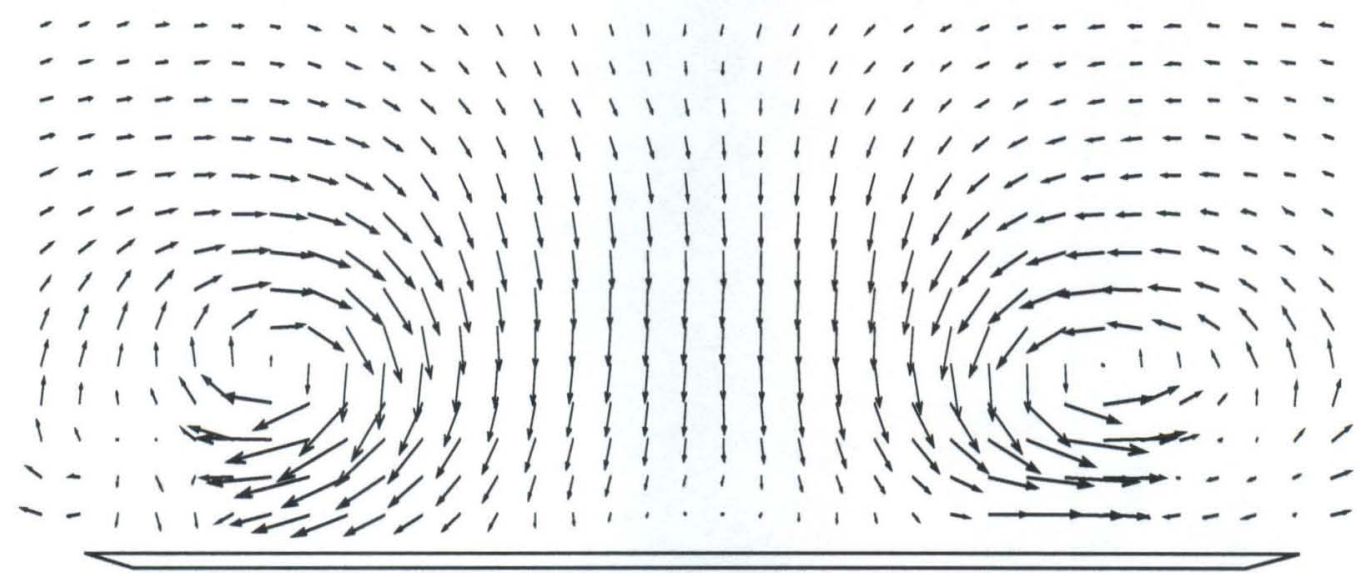

Figure 2.10. Typical full-span SPIV data, streamwise view

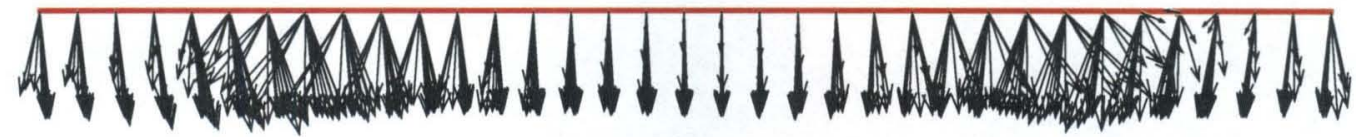

Figure 2.11. Full span SPIV data, planform view

Figure 2.10 shows the view looking upstream over the leeward side of the wing.

Flow is out of the page, and a cross-sectional cut of the wing itself is shown at the bottom of the figure. Figure 2.11 shows the same data, but from the planform view, looking down one the leeward surface of the wing. The free stream flow is in the direction toward the bottom of the page. The swirling motions characteristic of the primary LEVs cores are at least qualitatively reasonable. But secondary LEVs are not resolved, and neither is the leading edge shear layer structure.

A similar data set was taken at $\mathrm{z} / \mathrm{c}=1.1$, just downstream of the trailing edge, and at the $15^{\circ}$ angle of attack, that is approximately at the downstream location of the region of vortex breakdown.

From these two figures, one can extract, for example, the axial and azimuthal velocity profiles along a spanwise line passing through the LEV core centers. In the 
following two figures, the plotted velocity components are normalized by the free stream velocity $(80 \mathrm{~mm} / \mathrm{s})$, as is the usual practice in the presentation of such data.

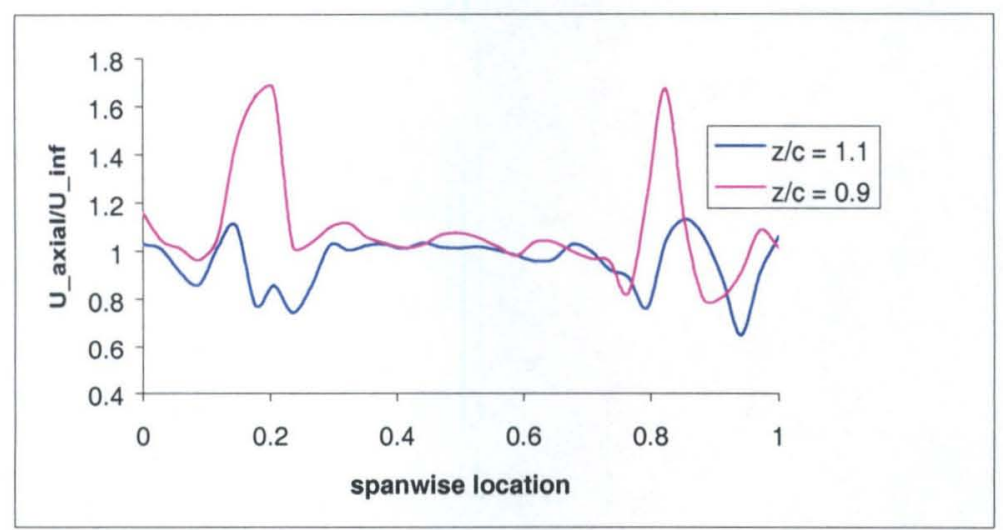

Figure 2.12. Normal velocity component along line passing through LEV cores

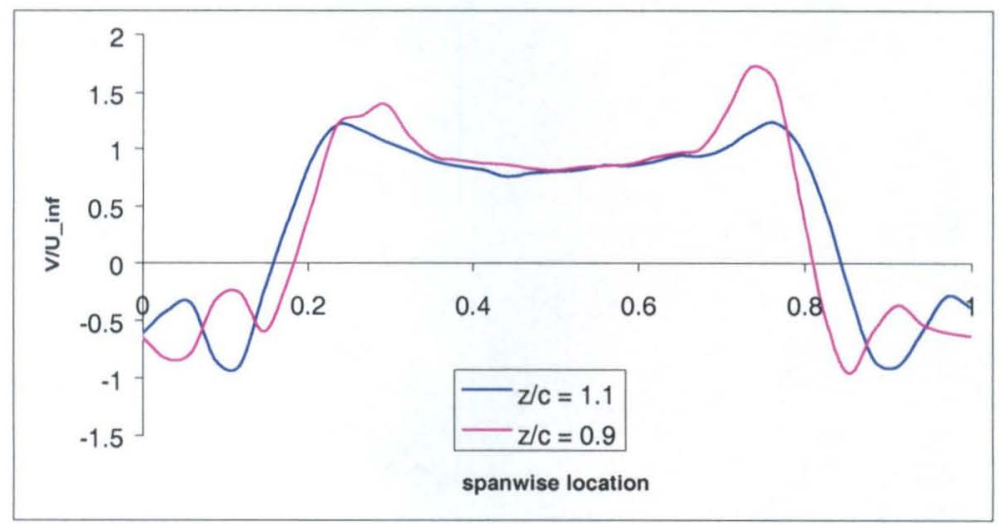

Figure 2.13. Azimuthal velocity along line passing through LEV cores

From Figure 2.12 and Figure 2.13, we can conclude that the full-span SDPIV measurement registered the normal component in main cores with reasonable verisimilitude, albeit better resolution is necessary. From Figure 2.13, the viscous "subcore" appears to be around 0.08 span units in diameter, as measured in peak-to-peak velocity ramps. Comparing the two figures, some smearing and loss of coherent axial flow with onset of VB is seen in going from $\mathrm{z} / \mathrm{c}=0.9$ to $\mathrm{z} / \mathrm{c}=1.1$. This is subsequently confirmed by flow visualization (Chapter 4). 


\subsection{The 3D vorticity field}

It is to be emphasized that while stereo PIV gives all three velocity components, it has no immediate advantage over regular PIV in giving the three vorticity vector components, since its domain of measurement is still planar. Only the out-of-plane vorticity component, $\omega_{z}=\partial v / \partial x-\partial u / \partial y$, is available. However, the in-plane vorticity components - or more directly, the out-of-plane velocity gradients, can be calculated from a differencing scheme, if one has available adjacent planes of 3-component velocity data. Here "adjacent" means "close enough in streamwise separation to give a robust differencing result."

A simple method of computing all three components of vorticity is suggested. This is similar to the 2-D method of Willert ${ }^{56}$, where one computes line integrals around an in-plane $3 \times 3$ computational stencil, and then divides the integrated quantity by the enclosed area, thus obtaining the component of vorticity normal to the plane of the contour. In the 3-D extension, a triplet of planes of velocity data is required, as shown schematically in Figure 2.14.

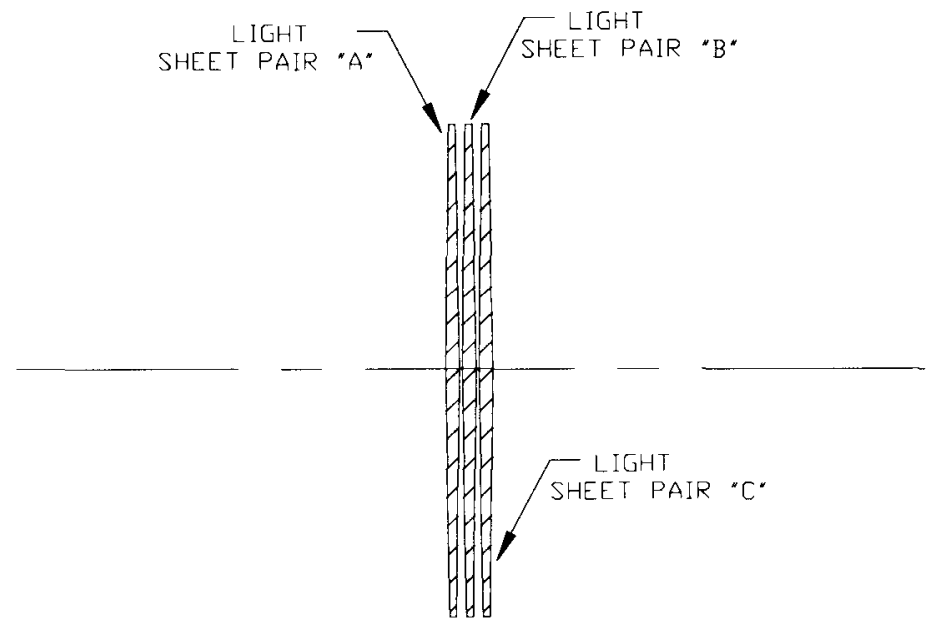

Figure 2.14. Arrangement of triplet of adjacent interrogation planes 
Because data sets are recorded in one plane at a time, it is not possible to make an "instantaneous" relation between the different planes. An attempt to compute 3component vorticity from instantaneous (that is, from a single image pair) will introduce a spurious time dependency. Thus, vorticity can only be computed in an averaged sense, i.e., from averaged data in the three planes. "Instantaneous" 3-component vorticity data could in principle be obtained if the light sheet were swept across the model surface, such as by a rotating mirror arrangement.

The three planes of data allow one to assemble a $3 \times 3 \times 3$ computational stencil (Figure 2.15).

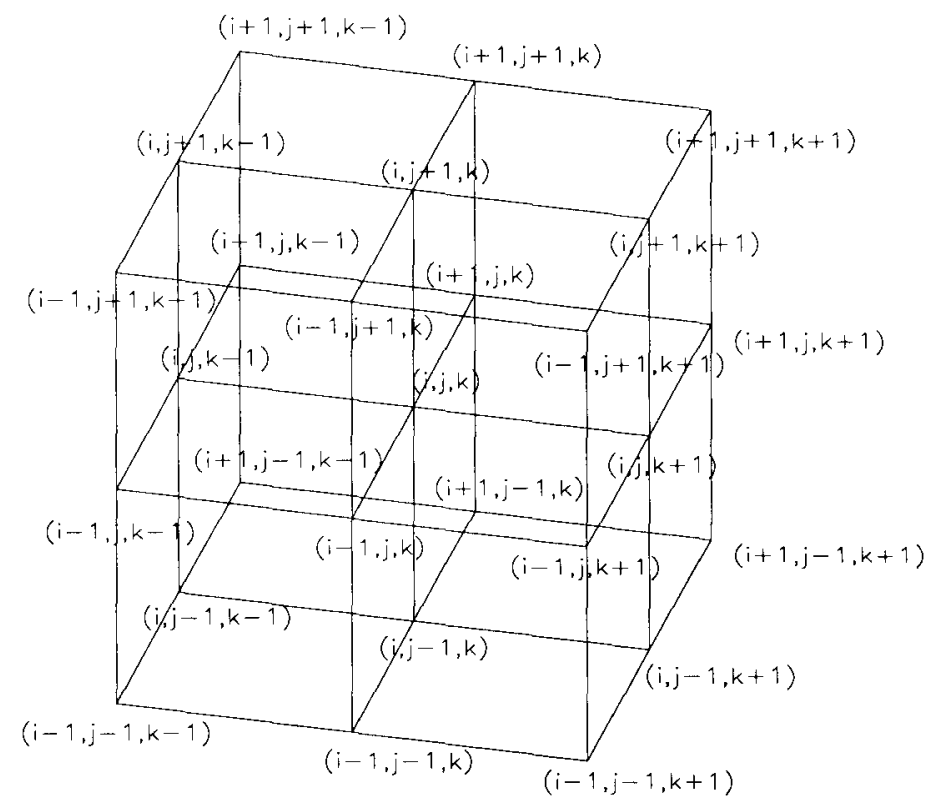

Figure 2.15. 3-D computational stencil of $3 \times 3 \times 3$ velocity nodes

Following the notation of Figure 2.15, the three components of vorticity,

$$
\begin{aligned}
& \omega_{z}=\omega_{z}(u(x, y, z), v(x, y, z)), \\
& \omega_{y}=\omega_{y}(u(x, y, z), w(x, y, z)), \\
& \omega_{x}=\omega_{x}(v(x, y, z), w(x, y, z))
\end{aligned}
$$

are given by the difference relations: 


$$
\begin{aligned}
& \omega_{X} \approx \frac{\Gamma_{k, j}}{4 \Delta z \Delta y}=\left(w_{i, j-1, k-1}+w_{i, j-1, k}\right) \frac{\Delta z}{2}+\left(w_{i, j-1, k}+w_{i, j-1, k+1}\right) \frac{\Delta z}{2}+ \\
& +\left(v_{i, j-1, k+1}+v_{i, j, k+1}\right) \frac{\Delta y}{2}+\left(v_{i, j, k+1}+v_{i, j+1, k+1}\right) \frac{\Delta y}{2}+ \\
& -\left(w_{i, j+1, k+1}+w_{i, j+1, k}\right) \frac{\Delta z}{2}-\left(w_{i, j+1, k}+w_{i, j+1, k-1}\right) \frac{\Delta z}{2}+ \\
& -\left(v_{i, j+1, k-1}+v_{i, j, k-1}\right) \frac{\Delta y}{2}-\left(v_{i, j, k-1}+v_{i, j-1, k-1}\right) \frac{\Delta y}{2} \\
& \omega_{Y} \approx \frac{\Gamma_{k, i}}{4 \Delta z \Delta x}=\left(w_{i-1, j, k-1}+w_{i-1, j, k}\right) \frac{\Delta z}{2}+\left(w_{i-1, j, k}+w_{i-1, j, k+1}\right) \frac{\Delta z}{2}+ \\
& +\left(u_{i-1, j, k+1}+u_{i, j, k+1}\right) \frac{\Delta x}{2}+\left(u_{i, j, k+1}+u_{i+1, j, k+1}\right) \frac{\Delta x}{2}+ \\
& -\left(w_{i+1, j, k+1}+w_{i+1, j, k}\right) \frac{\Delta z}{2}-\left(w_{i+1, j, k}+w_{i+1, j, k-1}\right) \frac{\Delta z}{2}+ \\
& -\left(u_{i+1, j, k-1}+u_{i, j, k-1}\right) \frac{\Delta x}{2}-\left(u_{i, j, k-1}+u_{i-1, j, k-1}\right) \frac{\Delta x}{2} \\
& \omega_{z} \approx \frac{\Gamma_{i, j}}{4 \Delta x \Delta y}=\left(u_{i-1, j-1, k}+u_{i, j-1, k}\right) \frac{\Delta x}{2}+\left(u_{i, j-1, k}+u_{i+1, j-1, k}\right) \frac{\Delta x}{2}+ \\
& +\left(v_{i+1, j-1, k}+v_{i+1, j, k}\right) \frac{\Delta y}{2}+\left(v_{i+1, j, k}+v_{i+1, j+1, k}\right) \frac{\Delta y}{2}+ \\
& -\left(u_{i+1, j+1, k}+u_{i, j+1, k}\right) \frac{\Delta x}{2}-\left(u_{i, j+1, k}+u_{i-1, j+1, k}\right) \frac{\Delta x}{2}+ \\
& -\left(v_{i-1, j+1, k}+v_{i-1, j, k}\right) \frac{\Delta y}{2}-\left(v_{i-1, j, k}+v_{i-1, j-1, k}\right) \frac{\Delta y}{2}
\end{aligned}
$$

where $\Delta x, \Delta y, \Delta z$ are half of the edge length of the computational stencil in the $\mathrm{x}, \mathrm{y}$, and $\mathrm{z}$ directions, respectively. The z-direction is, as usual, in the out-of-plane direction. Thus, the index $k$ only takes on the values $1,2,3$, whereas $i$ and $j$ traverse the PIV interrogation domain, window by window.

Since the scheme is based on central differencing, it should be nominally secondorder accurate. 


\subsection{Summary}

As pointed out by Prasad ${ }^{51}$, analysis of the error of the calibration of SPIV - that is, the effect of calibration grid orientation with respect to the laser light sheet, of the dewarping mappings of the calibration grid, etc., remains the main source of doubt regarding the robustness of SPIV. Validation tests with an artificial particle speckling and measurement of the free stream flow provided some insight into the limitation of SPIV. The artificial particle speckling tests need to be rigorously pursued, and extended to encompass rotational motions as well as translations.

The parallax error of SPIV encountered in the present experiment remains a vexing and unexplained error, not found by any other workers in the field. Fortunately, the erroneous velocity field introduced by the SPIV measurements is irrotational, and small in magnitude. It can be "corrected" with a simple additive procedure.

SPIV applied to a full-span measurement of the delta wing flow field revealed the capacity to capture the main features of the flow, as well as the need to zoom in to capture more detail with the presently available digital video camera resolution.

SPIV in its original form offers no further insight into the in-plane vorticity components, because out-of-plane velocity gradients are not available. A triple-plane arrangement was suggested, which allows for an estimate of all three components of vorticity by means of central differencing in the out-of-plane direction. While rather cumbersome and potentially inaccurate, this technique is a useful tool in conceptual investigations, and is considered again for a select number of test cases in Chapter 5. 


\section{Chapter 3 Experimental Setup}

This chapter describes the physical implementation of the techniques discussed in the previous chapter. The testing facility and wing models are described, and some practical issues of digital particle image velocimetry - stereo or two-dimensional - are briefly reviewed.

\subsection{Justification use of a water tunnel}

One finds in the literature experiments on delta wing flow fields conducted both in wind tunnels and water tunnels. The former is of course the natural choice for flows of aerodynamic interest, but the latter has some interesting advantages. These include:

1. Stable flow at low speeds, down to $30 \mathrm{~mm} / \mathrm{s}$.

2. Low diffusion advantageous for flow visualization by dye injection.

3. Neutral buoyancy issues of PIV tracker particles (see 3.5.4).

4. Brighter laser scattering from tracker particles, reducing need for high laser power.

5. Minimal disturbance due to mechanical vibrations, since the facility runs at such low speed.

(1) is useful in constructing flows in nominally laminar conditions, where

Reynolds numbers on the order of $10^{4}$ are typical. As noted in Chapter 1, this has the useful consequence of allowing one to construct a more reliable comparison between the experimental data and the laminar theoretical models, with the nominal confidence that discrepancies between theory and experiment are not due to the insidious complications of turbulence.

(5) is of some importance in minimizing flow "turbulence," where turbulence refers not only to undulations due to the intrinsic flow physics, but also due to external influences such as the tunnel motor and pump, and the structural response of the test 
section walls. The tunnel used in these experiments was built especially with these issues in mind.

It is perhaps worth mentioning some previous work on delta wings in water tunnels. Erickson ${ }^{57}$ presents data taken in a vertical water tunnel with freestream speeds of $0.25-0.35 \mathrm{ft} / \mathrm{s}(76-107 \mathrm{~mm} / \mathrm{s})$, supporting the idea that flows with separation over sharp leading edges are qualitatively similar over a very broad range of Reynolds numbers, from $O\left(10^{4}\right)$ to full scale. More specifically, the strength of the leading edge vortex sheet behaves in accordance with this result. However, such is not the case for viscositydominated features such as the surface leeward surface boundary layer and the secondary separation, and the LEV inner core region. Erickson comments that for the higher angles of attack, where the general leeward side flow is dominated by a strong adverse pressure gradient, water tunnel to wind tunnel (and flight test) similarity is quite good. But "at low angles of attack, where vortex core/boundary layer interaction can be significant," the similarity is less clear ${ }^{58}$. This is illustrated dramatically in the stereo PIV data of Chapter 5, and further discussed in Chapter 6.

Poisson-Quinton and Werle ${ }^{59}$ describe the particular advantages of visualization of the delta wing flowfield in a water tunnel environment, such as the low diffusion of injected dye. Thompson ${ }^{60}$ measured the vortex breakdown location over a series of delta wings with $65^{\circ}$ and greater leading edge sweep, with the usual conclusion that breakdown location moves upstream with decreasing sweep or increasing angle of attack. The work of Miau et al ${ }^{45}$ was also conducted in a water tunnel, at Reynolds number of 7000. Experiments on nonslender delta wings in water tunnels, as in wind tunnels, are relatively rare. 


\subsection{Description of the water tunnel}

As a consequence of materiel constraints, rather than a specific scientific purpose, a new water tunnel was constructed as part of the present experimental work. This facility, shown in Figure 3.1 and described further in Appendix A, features an 18 "x24"x96" (457x610x2438 mm) test section, with a free surface open to the atmosphere. The presence of the free surface, almost universal among horizontal-circuit low speed water tunnel designs, is not specifically utilized in this study, though one could consider the intriguing problem of LEVs of a surface-piercing delta wing and the resulting deformation of the free surface. Rather, the open top is used as a convenience to position the model and to adjust the optics and dye injection probe. Tunnel operating speed can be varied from approximately 30 to $500 \mathrm{~mm} / \mathrm{s}$.

However, free surface effects at first also manifested themselves as a shallow water free surface "sloshing" in the tunnel; see Appendix B. This was attenuated by means of sealing the top of the tunnel in the intake plenum and contraction sections. 


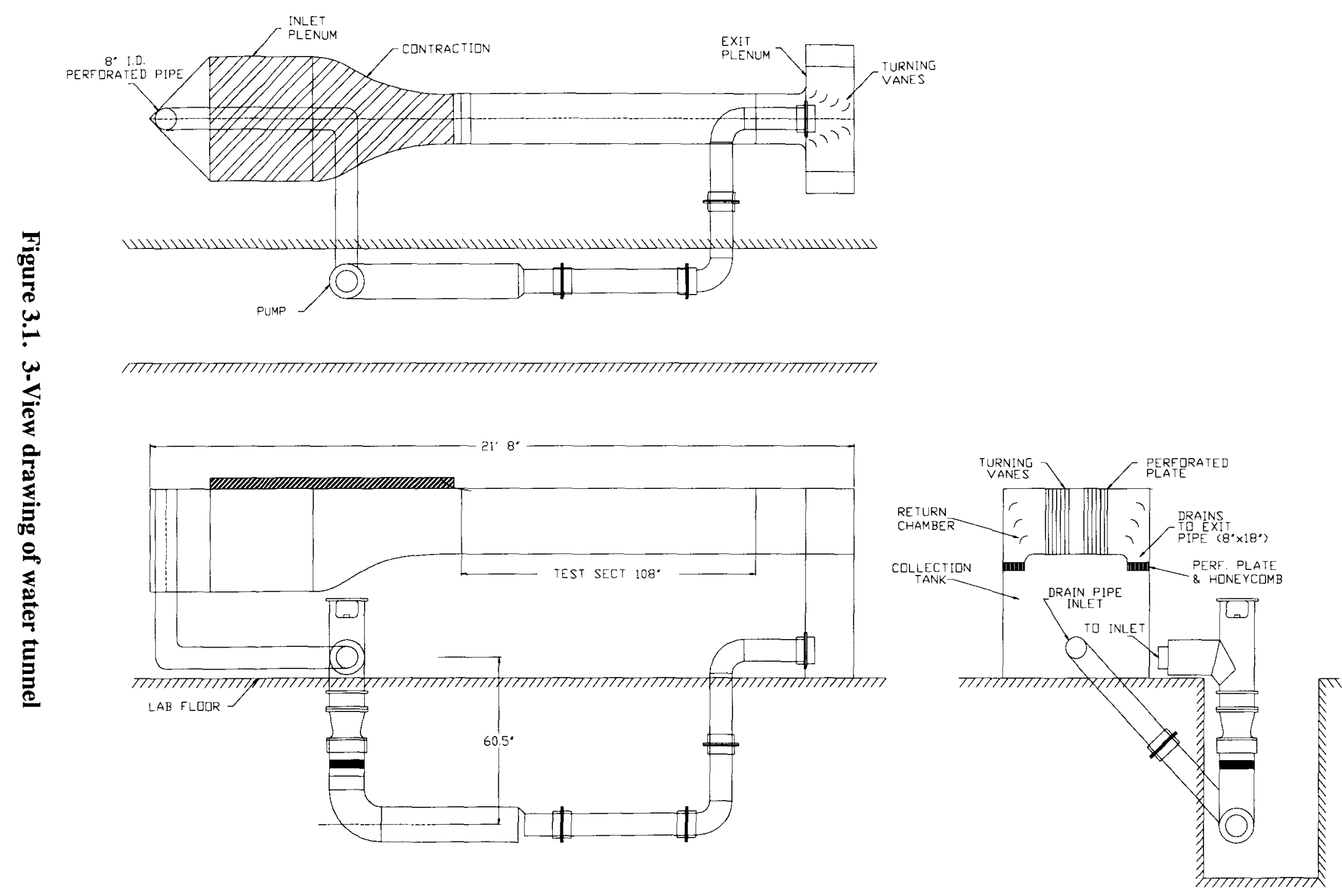




\subsection{The wing models}

\subsubsection{Description}

Two wing models were used in this study. This first is a "baseline" case, with $65^{\circ}$ leading edge sweep. The choice of such a geometry was motivated in Chapter 1 . The second model was selected to be as nonslender as possible, while still retaining evidence of stable LEV-type structures over at least part of the planform. Based on considerations outlined in Chapter 1 , a sweep of $50^{\circ}$ was selected.

The models, shown in Figure 3.3 and Error! Reference source not found., both had an $180 \mathrm{~mm}$ span and thickness of $0.125 "(3.175 \mathrm{~mm})$, resulting in a thickness-tochord ratio of 0.0296 for the $50^{\circ}$ wing (root chord: $107 \mathrm{~mm}$ ) and 0.0165 for the $65^{\circ}$ wing (root chord: 193mm). Common span was selected in favor of common chord, in consideration of blockage effects in the test section, and physical scaling effects with respect to a fixed camera field of view. Both wings were beveled $30^{\circ}$ on the windward side, and flat on the windward side.

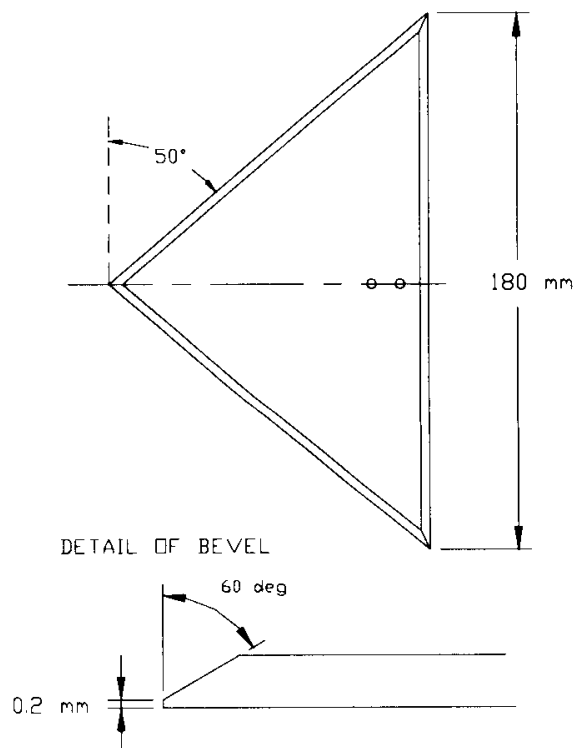

Figure 3.2. $50^{\circ}$ wing 


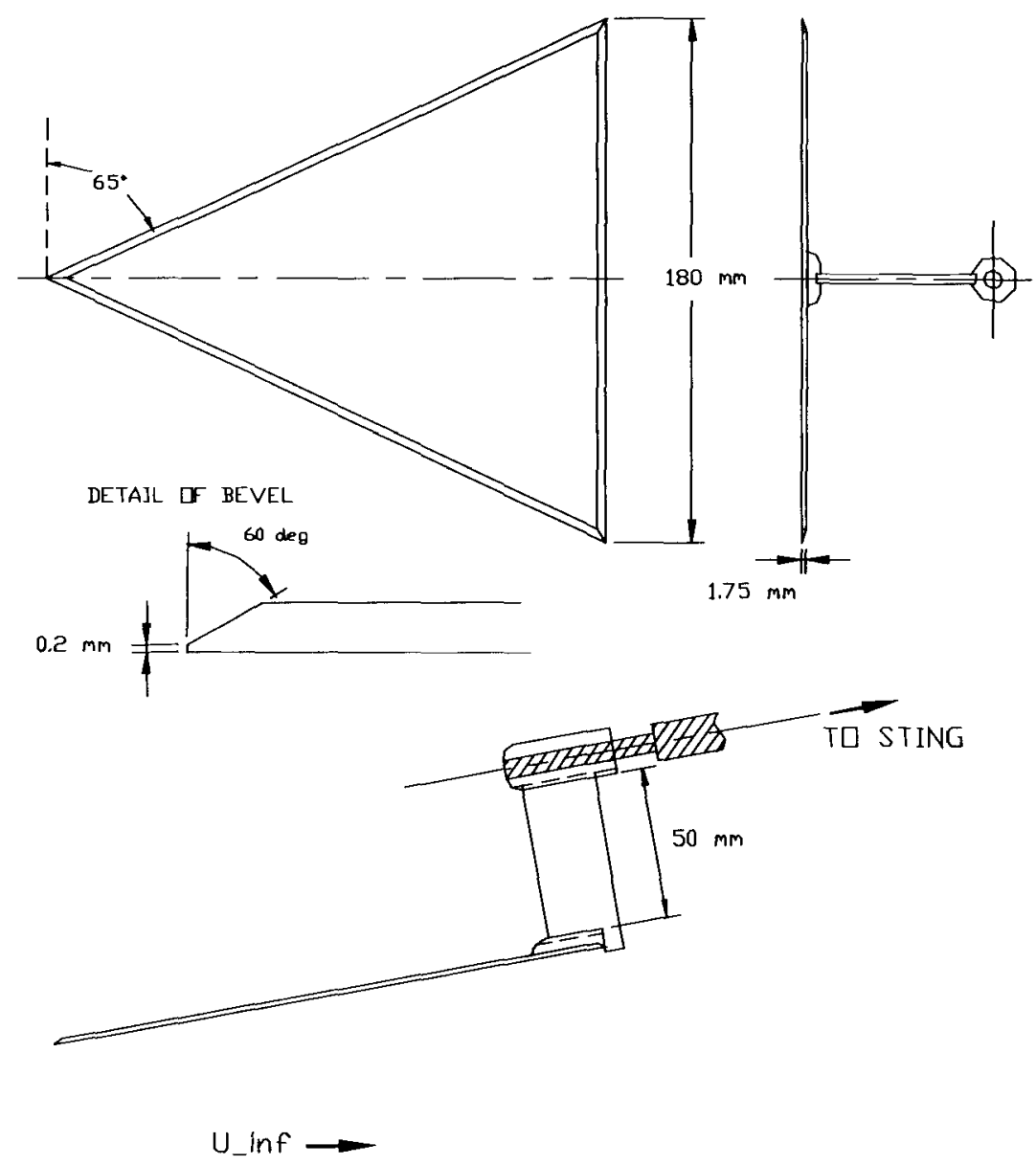

Figure 3.3. $65^{\circ}$ wing, with mounting arrangement

\subsubsection{Some design issues}

The wings were machined from Plexiglas. Plexiglas is advantageous from the point of view of flow visualization and PIV. While it is not essential to use a "transparent" material, since the model is mounted upside down and viewed from the bottom of the tunnel, a transparent model tends to reduce unwanted reflections of the light sheet, as well as to increase viewing flexibility. However, Plexiglas is difficult to machine accurately at the sharp leading edges. A leading edge thickness of $0.005 " \pm 0.003$ " was achieved by supporting the model during machining on a bed of cast 
wax, while the cutting itself was made with a "fly cutter" on a milling machine. The normal beveling procedure, with an endmill, resulted in the inevitable chipping of the model at the leading edges, especially at the apex.

A symmetric model, while lacking a flat top or bottom surface, has the advantage of zero camber, useful in the bookkeeping of the relative importance of potential and vortical lift contributions, since the geometric zero angle of attack is theoretically also the aerodynamic zero angle of attack. The choice of symmetrical mounting and associated sting geometry lends itself well to tests with variable roll angle, or indeed, with a dynamic roll rig. And as noted in Chapter 1 , the choice of beveling geometry can have a significant affect of the strength of the LEVs, especially for the less slender wings. In fact, the choice of $50^{\circ}$ sweep for the second model was also influenced by flow visualizations conducted for a third model, with $45^{\circ}$ sweep and crudely cut symmetrical bevels. The latter model had little discernable LEV-type structure, and hence, the $50^{\circ}$ model was pronounced to be a limiting case. But as evidenced from systematic experiments with the $50^{\circ}$ model in the present investigation, $50^{\circ}$ sweep is probably not the limiting case, when consistent choice of bevel geometry is taken into account (see, for example, ${ }^{45}$ ).

However, it was optical - and not aerodynamic - requirements that drove the final model designs. Initial experience with a symmetrically beveled $65^{\circ}$ model showed that the leeward bevels produced extensive reflections of the laser light, contaminating the image in the camera ccd arrays. Theoretically the best solution to this problem would be to match the indices of refraction of the material from which the models are made, and of the working fluid - for example, by mixing a fluid with high index of refraction with the 
water. This, however, is prohibitively expensive for a relatively large facility (total volume of approximately 3000 liters).

In all except one case, noted below, testing speed was $80 \mathrm{~mm} / \mathrm{s}$, corresponding to a Reynolds number of 8500 and 15400 for the $50^{\circ}$ and $65^{\circ}$ wings, respectively (based on root chord). The choice of flow speed was driven by practical considerations as well as by the desire to have nominally laminar flow, for purposes of comparison to theoretical models that assume a laminar LEV subcore. For speeds above around $150 \mathrm{~mm} / \mathrm{s}$, it was difficult to introduce enough dye flow to obtain sufficient optical contrast between the dye streak and the background. For speeds below about $50 \mathrm{~mm} / \mathrm{s}$, the dye stream was very difficult to throttle to maintain a steady flow. To the extreme of either of these cases, an attempt at maintaining good optical quality risks disturbing the actual flow with the presence of the dye jet exiting the probe. The PIV results are much less sensitive to testing speed, since short-term particle concentration can be increased by briefly running the tunnel at full speed to "mix" the particles resident throughout the tunnel circuit; the inverse effect is obtained by running the tunnel at low speed for several hours.

\subsection{Sting and model mounting system}

A special variable AOA sting was constructed specifically for the present study. The sting involves no particular innovation, but was custom designed for reasons of lack of availability of an existing device, and for fitting to the new tunnel. The sting (see Figure 3.4), constructed of 304 stainless steel, features a degree wheel and locking mechanism for setting AOA, and a vertical lead screw for repositioning the model such that it would remain near the center of the test section regardless of the angle of attack. Roll angle is adjustable by rotating the model support rod within the sting arm, although 
in the present experiments, zero roll angle was set for all cases. The mounting of a model in the test section, along with the light sheet formation and imaging optics, is shown in Fig. 4.4.

A "foot" mounted to the end of the support rod lowers the model 2" below the sting arm centerline (see Figure 4.2). This has several advantages, for example, for better optical access to the flow over the leeward side when viewed from downstream, and for greater support to the claim that the model mounting mechanism has at most minor upstream influence on the flowfield over the wing. The entire assembly is hung from a carriage that rides on rails above the test section, thereby allowing for optimal accommodation of the model in the test section in the streamwise direction, and for translating the model with respect to the fixed laser light sheet. In addition, this carriage can in principle be connected to a horizontal lead screw and stepper motor, converting the test section into a tow tank, with or without concurrent operation of the tunnel water circuit. 


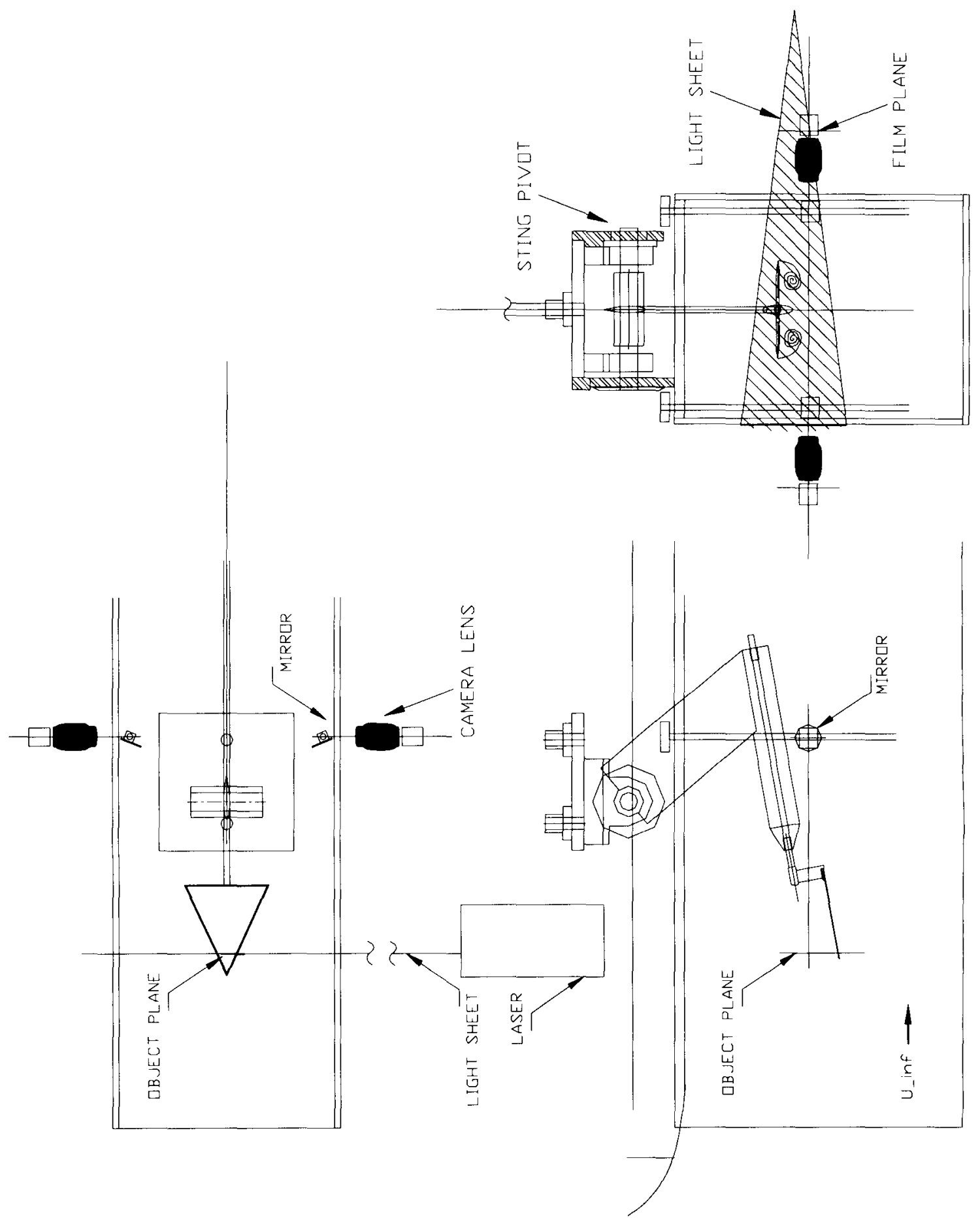

Figure 3.4. 3-View drawing of test section, model mounting and optics

\subsection{PIV imaging system}

\subsubsection{Conceptual basis}


The imaging arrangement of a DPIV system consists of a method of generating a planar light sheet, tracer particles that scatter light as they pass through this light sheet, a camera setup that records the light scattered by the tracer particles, an image acquisition system, and a timing mechanism that paces the various electronic components of this system. The present experiments differ from what has become something of a canonical arrangement of video-based PIV (see Willert and Gharib ${ }^{65}$, and Park ${ }^{61}$ ) mostly in terms of new requirements arising from the simultaneous use of two cameras.

The underlying 2-D version of PIV applied in this study is an evolution of the technique of Willert and Gharib ${ }^{65}$, which is implemented with a CCD video camera viewing tracker particles convecting through (or across) a laser light sheet. The sequence of video frames is delimited into A-B image pairs, and images are discretized into "windows." Image A is cross-correlated with B on a window-by-window basis. Location of the cross-correlation peak corresponds to the local displacement vector, with origin centered about the window center. The important but by now standard methods of achieving sub-pixel accuracy, rejection and substitution of "outliers" (apparent displacement vectors that are clearly erroneous), smoothing, etc., are described in Raffel et al. ${ }^{64}$. Window shifting, a highly successful error reduction technique based on iterative application of PIV ${ }^{62}$ was applied in the post-processing. The actual computer codes used to accomplish these tasks are described by Jeon ${ }^{63}$.

Specifics of the hardware and software of the stereo DPIV system are described below.

\subsubsection{Laser light sheet optics}


In each frame, the image recording event corresponds to the brief interval of time during which the laser actually fires. The time difference between laser pulses is set according to the needs of the experiment, and is not limited by the camera framing rate per se. A New Wave Research "Minilase II" $50 \mathrm{~mJ} /$ pulse Nd:YAG laser is used to illuminate the field of view. This is a twin pulsed laser, with each laser firing at a (maximum) rate of $15 \mathrm{~Hz}$, with pulse duration of about $9 \mathrm{~ns}$. This matches the NTSC camera framing rate of 30 frames/second. An internal system of "combining optics" merges the beams emanating from the two laser heads. By appropriate intentional misalignment of the combining optics, one can set the two beams at an arbitrary offset from one another. As will be described below, this offset is used to improve PIV correlation by capturing a higher percentage of particles passing through the light sheets.

The light sheet forming optics consist of two spherical and two cylindrical lenses. The arrangement is shown in the following optically inaccurate cartoon (Figure 3.5).

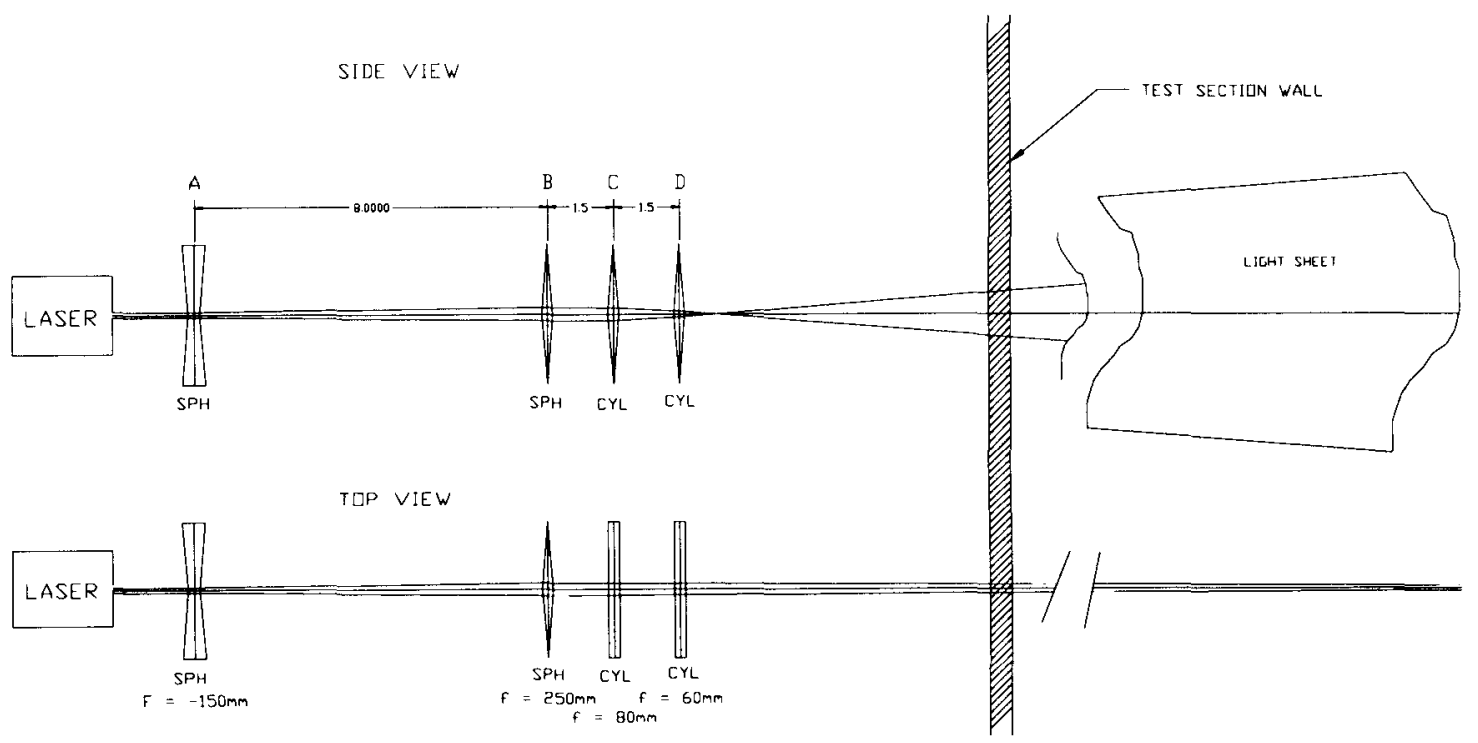

Figure 3.5. Schematic of light sheet forming optics 
The system is similar to that suggested by Raffel et al ${ }^{64}$. Varying the separation between the cylindrical lenses is the primary means of controlling the light sheet width, while varying the separation between the second spherical lens and the first cylindrical lens is the primary means of controlling light sheet thickness. It should be noted that no attempt has been made to collimate the light. Nevertheless, the thickness of the light sheets is essentially constant across the interrogation region, because that region is so small $(33 \times 25 \mathrm{~mm})$ compared to the distance from the final lens $(\sim 400 \mathrm{~mm})$. Minor variations of light intensity are acceptable, since the available intensity is typically so much greater than required, and since the DPIV algorithm is highly tolerant of variations in illumination.

\subsubsection{Laser light sheet geometry issues}

For most applications of PIV, the natural way to align the laser light sheet is along the bulk flow direction. This minimizes the so-called out of plane loss of particles; some particles that were in frame A convect out of the light sheet by the time frame B is captured, and some particles that were not in the sheet during frame A, appear in frame B.

However, in the present implementation, the natural way to align the light sheet is normal to the delta wing LEV axes. To simplify matters, the light sheet was actually normal to the free stream direction. To reduce out-of-plane particle loss, the light sheet was stepped, with the sheet displaced slightly in the downstream direction during the B frame, with respect to its streamwise position in the A frame.

Images of the SPIV calibration grid were taken with the grid located at the midpoint between the two light sheets, as illustrated in Figure 3.6. In an attempt to 
address the issue of parallax error, the calibration grid was displaced for trial purposes in the streamwise direction, away from the midpoint between the light sheets. It was found that so long as the calibration plane remains within the depth of field of the camera lenses, the resulting SPIV measurement is not discernibly affected.

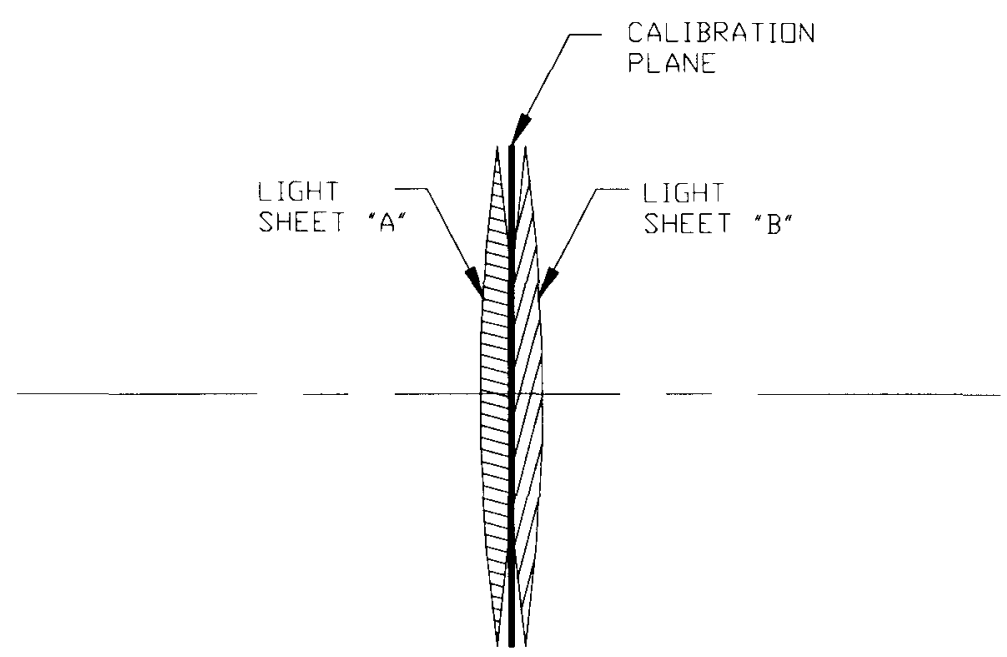

Figure 3.6. Dewarping plane and light sheet arrangement

The jet-like profile of axial velocity in the LEV core upstream of vortex breakdown further complicates the light sheet arrangement. If the flow velocity variations are minor increments about a bulk mean velocity (i.e., that of the free stream), then the centerplane-to-centerplane stepping of the light sheet to minimize out-of-plane particle loss is just the A-B time difference multiplied by expected bulk velocity. But the sharp variation in axial velocity across the LEV core makes the notion of bulk and incremental velocity vacuous.

The light sheet geometry was selected for a worst case scenario. For the velocity profiles in Figure 1.11, it is seen that the variations in axial velocity are on the order of twice the free stream velocity, while the in-plane velocity generally has a peak magnitude somewhat below that of the free stream. 
It was assumed that minimum in-plane speed is zero, and maximum is equal to $U_{\infty}$. In a certain $\Delta \mathrm{t}$, the maximum in-plane velocity corresponds to a particle displacement of $\mathrm{n}$ pixels. For a $32 \times 32$ interrogation window, $\mathrm{n}$ should be no more than about 10 , in order to avoid aliasing-type errors in the numerical implementation of DPIV (Willert ${ }^{65}$ ). And in that $\Delta \mathrm{t}$, the out-of-plane velocity convects the particles a maximum of $\left(3 U_{\infty}\right) \Delta t$ normal to the light sheet, or $3 \mathrm{n}$ pixels. This is the nominal minimum required total extent of light sheet thickness plus stepping. For a $640 \times 480$ pixel ccd array, there are about $80 \times 60$ (not independent) windows, assuming $32 \times 32$ windows with 8 pixel stepping in both coordinates. The light sheet thickness plus stepping, meanwhile, is about 30 pixels.

So changes in image magnification, by changing the physical extent of the visible subject region, have the same effect as changing the relative light sheet thickness. In the above, the light sheet thickness is fixed at about $1 / 20^{\text {th }}$ of the image horizontal extent. The choice of interrogation window size also affects the light sheet thickness, with larger windows requiring thicker light sheets. More precisely, a thick light sheet allows for a large interrogation window, because a larger range of allowable pixel displacements is available.

But the choice of flow speed does not affect the light sheet thickness - it only affects $\Delta t . \Delta t$ is selected so that the maximum in-plane particle displacement is indeed no larger than one-third of the window size.

In practice, light sheet thickness, spacing, and overlap are determined by opinion and experience, not rigorous evaluation. A "good combination" is one that produces the minimum number of outliers, both in the LEV core region and in other salient regions in 
the field of view, and which at the same time is as thin as possible. A thin light sheet is desirable to reduce spatial averaging of the PIV data in the out-of-plane direction, and to maximize laser light intensity.

Also for reasons of maximizing light intensity, the light sheet width should be kept as small as possible. To accomplish this, the light sheet was oriented along the spanwise direction with respect to the wing models (as opposed to the usual practice of shining the laser from below the test section) with the laser positioned at the side of the test section (Figure 3.4). This has the additional advantage of preserving fairly uniform light intensity through the region of interest. At the comparatively low angles of attack of interest in the present study, flow structures were expected to be near the wing surface, and thus, extending further laterally than vertically.

The choice of $8 \times 8$ pixel window stepping "improves" velocity vector density, at the expense of reducing the effectiveness of outlier rejection. With small window stepping, window-to window (and hence, vector-to-vector) interdependency is increased, and hence, isolated outliers tend to become multiplied into outlier "islands." These inhibit the outlier removal process, unless aggressive smoothing is invoked. But smoothing also attenuates the resolution of sharp gradients in the velocity field. It was in general not used in this study.

\subsubsection{Particles in the LEV core}

The problem of dearth of particle concentration inside the LEV cores is frequently encountered in laser-based measurements of vortical flows. One way that this was addressed in the present investigation was by using a mixture of particles with specific gravity ranging from greater than one to less than one-e.g., $0.9-1.1$, as in the present 
case. Without rigorous concern for the flow kinematics, it was conjectured that a mixture of particle with such variation in specific gravity would prevent particle loss in the viscous rotational regions of the flow, especially those in the primary LEV cores. As was seen subsequently, the entire flow region bounded by the delta wing leading edge shear layers was viscosity-dominated, but no obvious reduction of particle concentration in that region (as compared with the free stream) was observed.

\subsubsection{Cameras and camera triggering}

Stereo DPIV, with its requirement for two video cameras firing simultaneously, presents a new variation on the old problem of how to synchronize the events of camera triggering (that is, the initiation of the train of video frames), laser firing, and data acquisition.

The Pulnix TM-9701 ccd cameras used in this experiment have an accessible array of $480 \times 640$ pixels, digitized to 8-bit grayscale resolution. Each camera has an internal trigger, and it is this trigger that is used as the master event timer in video-based 2-D DPIV. However, when two cameras are used, their internal triggers would conflict, resulting in a train of video frames with a phase difference, which is seen as a "tracking" mismatch when viewed on a computer monitor. This problem is solved by the use of an external master triggering device (Sigma Electronics CSG-4500) that sends a synchronization signal to both cameras. This signal has two components, one for "horizontal drive" and one for "vertical drive." The former refers to the process of initiating a horizontal sweep of pixels in each row of the ccd array of each camera. The latter refers to the initialization of a new sweep of the ccd array, once the end of the 
bottom row of pixels is reached; this occurs twice in each frame, since the cameras are operating in interlaced mode.

\subsubsection{Optical filtering}

As seen in Figure 4.4, the right-side camera (viewing the Figure upside down) views the light sheet in an orientation of more forward-scattering than the left camera. Normally, scattering of laser light from the tracker particles is what the PIV process is supposed to record. Unfortunately, with one camera viewing the laser light sheet from the left of the test section, and another from the right, the intensity of light scattered by the tracker particles would be appreciably less on the left, since the camera on the right sees a more forward-type scatter cross section. Also, light reflected and distributed from the surface of the model, especially the bevels, tends to contaminate the particle images, saturating the ccd pixels. The alternative arrangement of shining the light sheet from the bottom of the test section has even worse reflection problems. Both of these problems were solved by using fluorescent tracker particles ${ }^{66}$ and photographic filters designed to block light below a certain frequency. The tracker particles contain rhodamine dye, which is excited by the frequency doubled Nd:YAG (shining at $532 \mathrm{~nm}$ ) to fluoresce at about $560 \mathrm{~nm}$. Kodak ${ }^{67}$ Wratten (gelatin) \#21 filters, having a sharp cutoff at around 540 $\mathrm{nm}$, were placed in front of the camera lenses. These filters conveniently admit most of the fluorescent light but block the laser and scattered light. Of course, all information due to scattering is lost. As compared with scattered light viewed with no filters, about 3 aperture stops of light intensity were lost. 


\section{Chapter 4 Flow Visualizations}

This chapter presents the results of the flow visualization experiments. Primary and secondary vortices resulting from leading edge separation for the $50^{\circ}$ wing are given principal emphasis, as wings of such modest sweep have received far less attention in the literature than the more slender wings. Flow visualization also gives semi-quantitative data on vortex breakdown location and axial flow speed in the core of the primary leading edge vortex.

\subsection{General description of flow visualization issues}

Flow visualization by injection of dye into the delta wing apex windward stagnation region (approximately $2 \mathrm{~mm}$ aft of the apex point) was conducted in order to observe the LEV core trajectory, the location and structure of vortex breakdown (VB), and the possible significance of secondary vortices, which lie outboard of the primary LEVs.

As mentioned previously, one of the principal advantages of "aerodynamics" experiments in a water tunnel is the relative convenience of carrying out flow visualization studies. In delta wing experiments, the injection of dye into the LEV core has by now become something of a classical technique of visualizing the core trajectory and onset of vortex breakdown. Dye is introduced from a probe located "near" the windward stagnation point just downstream of the wing apex. The meaning of "near" is ambiguous. Slight misalignment of the dye probe off the model centerline will result in all or nearly all of the dye entraining into only one of the vortex cores. In the present study, the dye probe was in some cases purposely displaced off center, to bias the 
injection into one of the LEVs and its accompanying secondary vortex, wherever applicable. In cases where left-right interaction was present, the bulk of the injected dye would occasionally "switch sides." In other cases an effort was made to place the dye probe to visualize both left and right cores. The geometric intersection of the windward LE bevels also plays a role in the behavior of the dye streak, though this too is ambiguous. Finally, it should be mentioned that too great a flow rate of dye at the lower model angles of attack, where radial pressure gradients (with respect to the LEV cores) are weak, will result in entrainment of the bulk of the dye along the centerline region of the wing, where the flow is nominally attached. However, at the higher angles of attack, where the LEVs are "unstable," dye will fill the "bulk separation" about the leeward side of the wing, rather than follow coherent streaklines.

\subsubsection{Core trajectory and vortex breakdown}

The most obvious use for dye injection is to visualize the LEV core (trajectories), upstream and to some extent downstream of their breakdown. As documented by various researchers, for slender wings, which nominally includes the $65^{\circ}$ wing, the dye trajectory is quite straight and steady upstream of breakdown. The breakdown itself is a "bubble" or "spiral" region (see for example Sarpkaya ${ }^{13}$ ), with an accompanying retardation of axial velocity and abrupt spreading of the core region. The presence of vortex breakdown is obvious from a "sudden" incoherence of the dye streak in the LEV core. This has been well documented for several decades.

For the $50^{\circ}$ wing, the same general ideas apply, though the LEVs are in general less well defined, and hence, the dye trajectories are more problematic to interpret. 
Suction in the LEV cores is weaker for the lower sweep wing, which complicates the technique of visualizing them with the dye.

\subsubsection{Dye injection issues: straight vs. wavy trajectories}

Besides illustrating the mean core trajectory, the dye streak also gives a qualitative estimate of undulations in the core. Here undulations are both those in time (wandering or bending of the core) and space (standing wave-type sinusoidal deformation of the core).

However, care must be taken in discerning whether a "wavy" dye streak represents a physical spiraling motion, or whether it is a mere figment of the experimental technique. In the latter case, misalignment of the dye probe can displace the dye injection some radial distance away from the LEV core, resulting in a corkscrewtype motion.

\subsubsection{Relation to PIV}

Dye injection gives a good indication of the VB location, and hence, how to schedule the location of the interrogation plane in the PIV tests. Second, the dye illustrates regimes of bulk unsteadiness, which again assists in the scheduling of PIV tests by suggesting how long to make the data record to capture the desired number of periods of oscillation (for periodic or quasi-periodic motion). Turning the dye injection on and off can yield information regarding the LEV core axial velocity, as described below. This makes an interesting comparison with the results of the PIV data. Finally, the side view and top view flow visualization images assist in locating the wing surface with 
respect to the flowfield in the PIV images, where of course the wing itself is not illuminated except by indirect inference.

\subsection{Flow visualization test matrix}

In a preliminary study of this nature, one is faced with the philosophical choice of whether to pursue an extensive test matrix, and thereby obscure the potential salient points in a swarm of data, or to streamline the data taking process, and thus possibly overlook pivotal test cases. The choice, barring the availability of overwhelming guiding evidence (the presence of which belies the term "preliminary study"), is in truth a matter of opinion. As outlined in Chapter 1, the present study is concerned with the flow regimes at relatively low angles of attack. The flow visualizations include the entire planform of the wings, extending approximately 1.5 root chords behind the apex, to give some indication of whether the entire flow field about the wing is stable. The PIV experiments, in contrast, focus on the near-apex region.

The cases tested in this investigation are summarized in Table 4-1. Each " $X$ " represents a sequence of 200 to 1000 image frames (depending on the observed presence of bulk unsteadiness), taken at the normal NTSC video rate of 30 frames per second.

\section{Table 4-1. Flow visualization test matrix} angle of 50 degree 65 degree attack, degwing wing

\begin{tabular}{|c|c|c|}
\hline 2.5 & $\mathrm{X}$ & $\mathrm{X}$ \\
\hline 5 & $\mathrm{X}$ & $\mathrm{X}$ \\
\hline 7.5 & $\mathrm{X}$ & $\mathrm{X}$ \\
\hline 10 & $\mathrm{X}$ & $\mathrm{X}$ \\
\hline 12.5 & $\mathrm{X}$ & $\mathrm{X}$ \\
\hline 15 & $\mathrm{X}$ & $\mathrm{X}$ \\
\hline 17.5 & & \\
\hline 20 & $\mathrm{X}$ & $\mathrm{X}$ \\
\hline
\end{tabular}




\begin{tabular}{|c|c|c|}
\hline 22.5 & & \\
\hline 25 & & $\mathrm{X}$ \\
\hline 27.5 & & \\
\hline 30 & & $\mathrm{X}$ \\
\hline 32.5 & & $\mathrm{X}$ \\
\hline 35 & & $\mathrm{X}$ \\
\hline
\end{tabular}

Beyond $20^{\circ}$ angle of attack, it was found that the flowfield about the $50^{\circ}$ wing has become sufficiently disorganized to render the flow field to no longer be of interest.

In these measurements, images were taken with two cameras. One was placed at the side of the tunnel test section, for the side view. The other was placed underneath the test section, to obtain the leeward planform view. In both cases, camera orientation was normal to the respective test section walls. In the planform view, the sting and dye probe to some extent obscure the images around the model centerline, but this region is of little interest anyway. The cameras were synchronized according to the same method as for the stereo PIV experiments.

In the following, representative images are shown, each in pairs (side view followed by planform view):

- $65^{\circ}$ wing: $5^{\circ}, 15^{\circ}, 25^{\circ}$.

- $50^{\circ}$ wing: $2.5^{\circ}, 5^{\circ}, 7.5^{\circ}, 10^{\circ}, 12.5^{\circ}, 15^{\circ}$, and $20^{\circ}$. Angles of attack illustrating strong secondary vortices and unsteady flow are given special attention.

Particular attention is given to unsteadiness and flow reversal in the $50^{\circ}$ wing.

The temporal evolution of the flow is documented in a series of frames.

\subsection{The $65^{\circ}$ wing images}

Results for the $65^{\circ}$ wing are largely classical in nature, and are presented for purposes of completeness. Of interest is the fact that stable, nearly symmetric LEV 
trajectories were observed for very low angles of attack, down to $5^{\circ}$ and to a lesser extent, even down to $2.5^{\circ}$.

A resume of sample cases is presented below. The dye injector probe, visible as a thick black line, is not to be confused with the flow features on the leeward side.

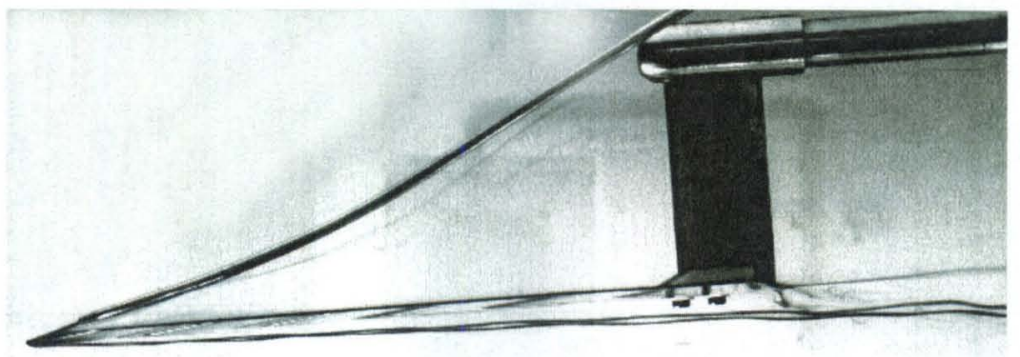

Figure 4.1. $\alpha=5^{\circ}$, side view

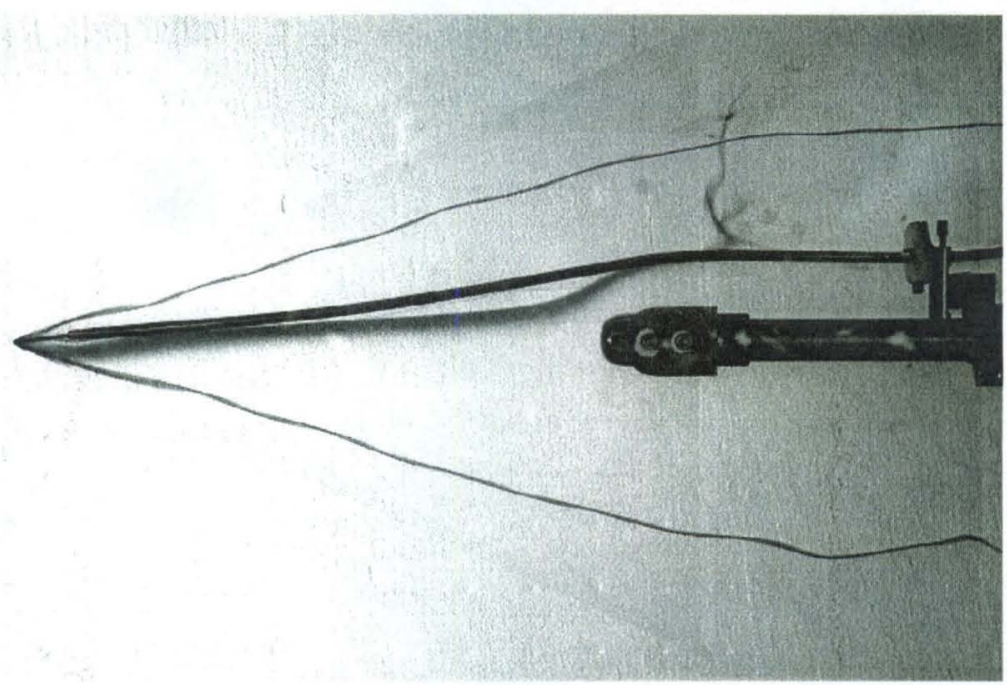

Figure 4.2. $\alpha=5^{\circ}$, planform view

At $5^{\circ}$ angle of attack, shown in Figure 4.1 and Figure 4.2, there is a slight waviness in the dye trajectory. This is probably attributable to the low radial pressure gradient about each LEV, and its resulting poor ability to keep the dye streak "focused." Nevertheless, it is clear that the LEV trajectories are nominally straight rays emanating from the wing apex. 


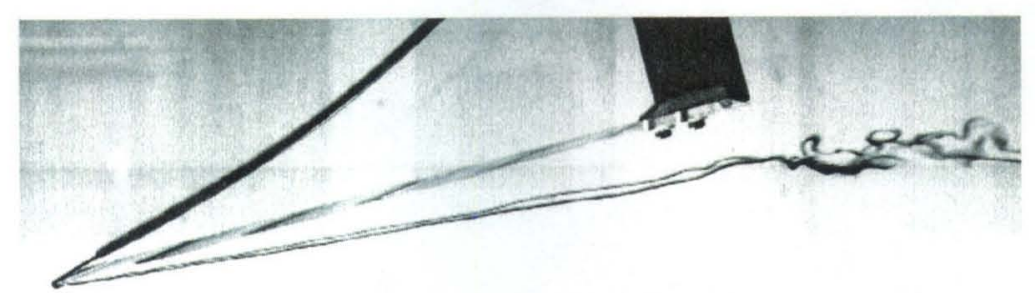

Figure 4.3. $\alpha=15^{\circ}$, side view

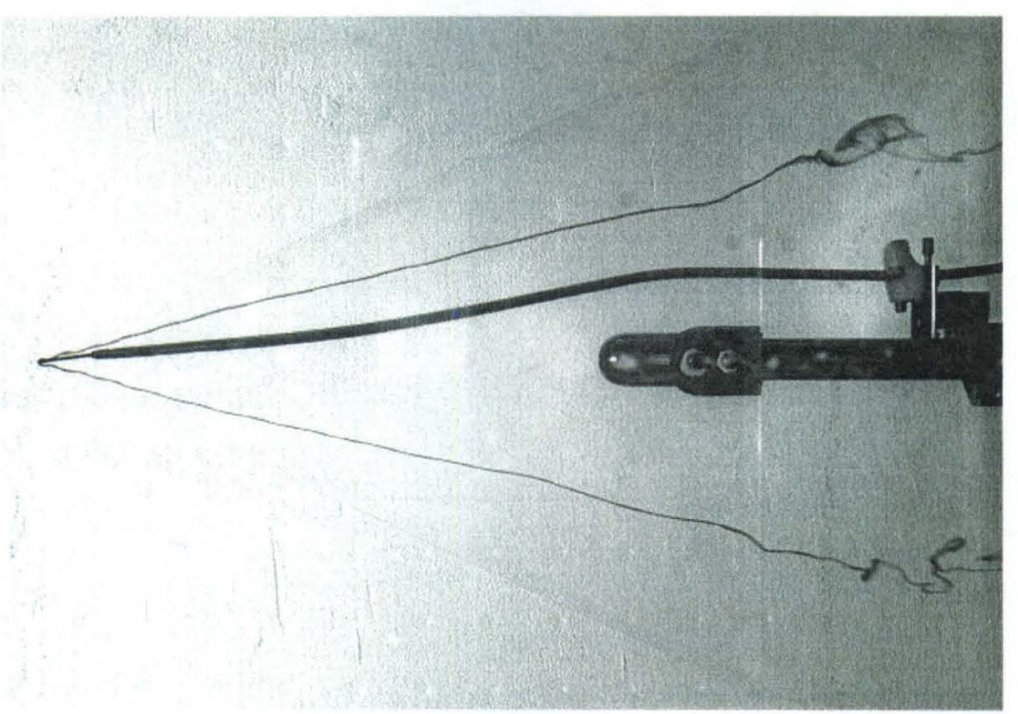

Figure 4.4. $\alpha=15^{\circ}$, planform view

At $15^{\circ}$ angle of attack (Figure 4.3 and Figure 4.4), waviness in the dye trajectories is largely absent.

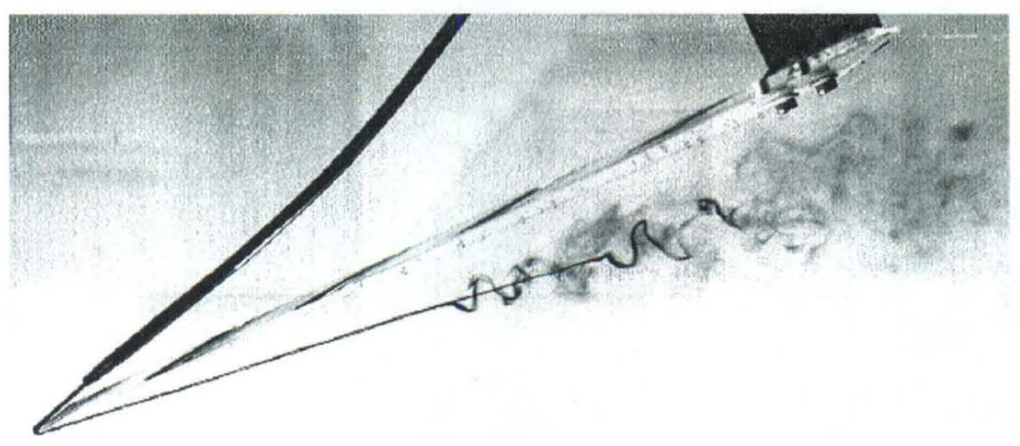

Figure 4.5. $\alpha=25^{\circ}$, side view 


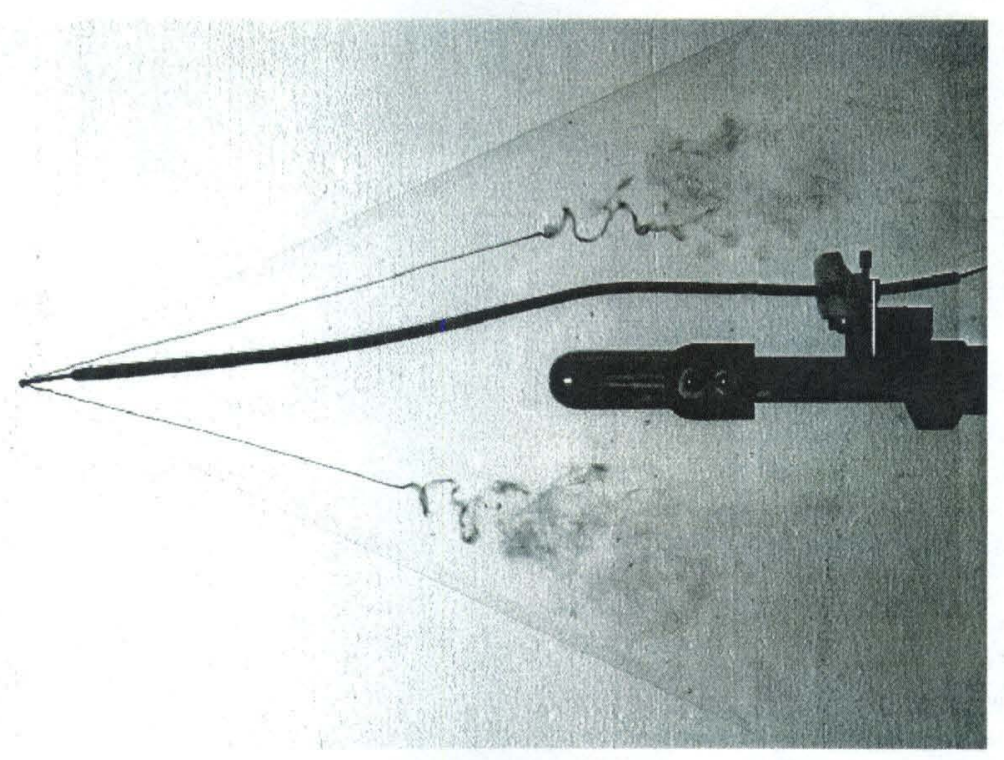

Figure 4.6. $\alpha=25^{\circ}$, planform view

By $25^{\circ}$ (Figure 4.5 and Figure 4.6), vortex breakdown has finally crossed the trailing edge. The actual crossing event occurs at or slightly below $20^{\circ}$. The dye streaks are very straight, sharply defined rays. This behavior continues with increasing angles of attack, limited of course by the eventual progression of breakdown to the apex.

Certainly up until $25^{\circ}$ angle of attack, the flow is quite steady upstream of breakdown. As will be seen shortly, this is quite in contrast with the scenario for the $50^{\circ}$ wing. A similar statement can be made regarding the observed presence of secondary vortices. These were in general weak, and difficult to capture with dye injection.

\subsection{The $50^{\circ}$ wing images}

The aspect ratio of the delta wing of $50^{\circ}$ sweep is 3.36 - well beyond the canonical range of "low aspect ratio wings." Thus, we would expect divergence of the experimental results from those predicted by slender wing theory, and a closer relation to 
the nominal flow characteristics of classical high aspect ratio wings, i.e., those adequately described by lifting line theory.

A major consequence of this is a "stall" at lower angles of attack than that for more slender wings. Indeed, flow visualization shows this to be the case. By $\alpha=20^{\circ}$, the LEVs are gone, and a side view of the flowfield clearly indicates a Kelvin-Helmholz separation over the leeward side. In other words, the leeward flow is in deep stall. Careful observation of the trajectory of a burst of injected dye reveals more detail of the flow structure.

At lower angles of attack, stable LEVs are present. At higher angles, the breakdown point moved rapidly upstream, consistent with the lower stall angle of attack of this nonslender wing.

\subsubsection{Presence of LEVs}

What might be considered stable, symmetrical LEVs were observed for angles of attack up to and including $15^{\circ}$. As for the $65^{\circ}$ wing, at $\alpha=2.5^{\circ}$, the smallest angle under investigation, a weak pair of LEVs was found. At $\alpha=5^{\circ}$, the LEVs extended to the trailing edge, and were very obvious. This situation persisted to $7.5^{\circ}$. From $10^{\circ}$ to $15^{\circ}$, the vortex breakdown point moved upstream from the trailing edge to a region of some proximity to the apex, where the precise location of breakdown was not obvious, due to unsteadiness (see below). 


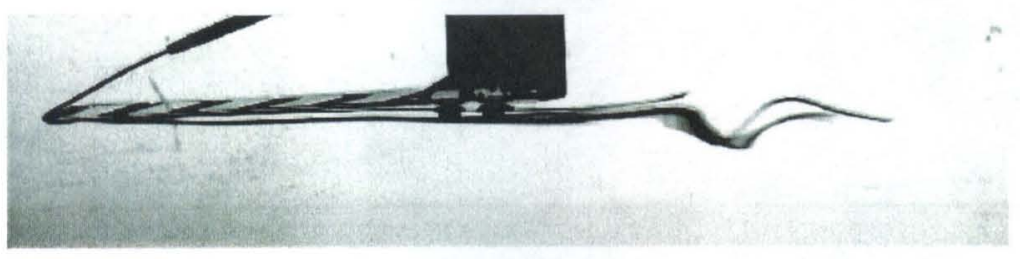

Figure 4.7. $\alpha=2.5^{\circ}$, side view

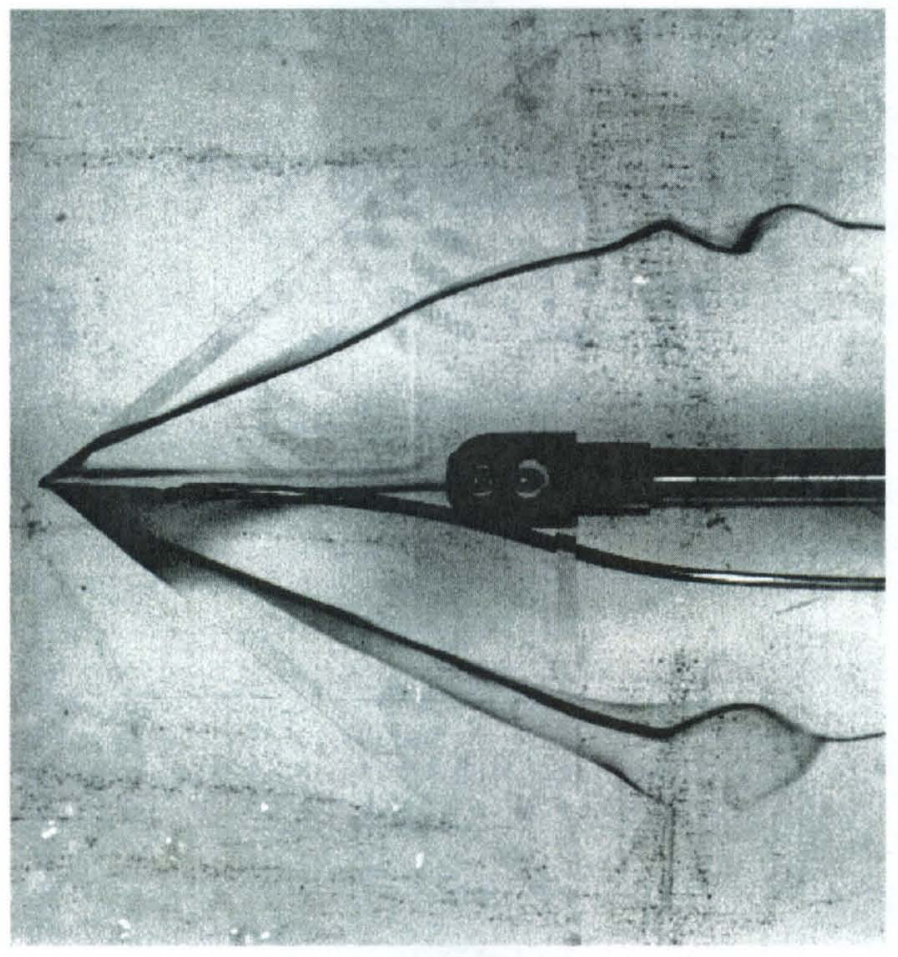

Figure 4.8. $\alpha=2.5^{\circ}$, planform view

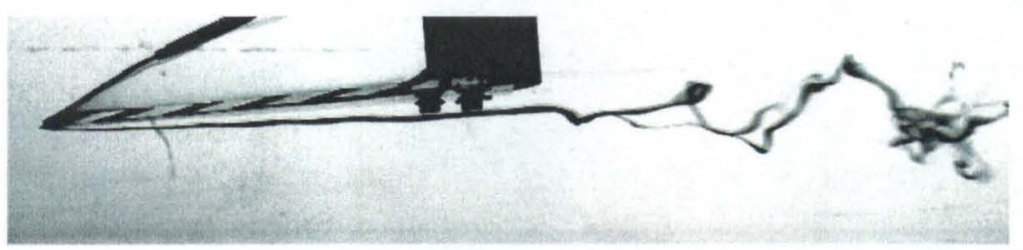

Figure 4.9. $\alpha=5^{\circ}$, side view 


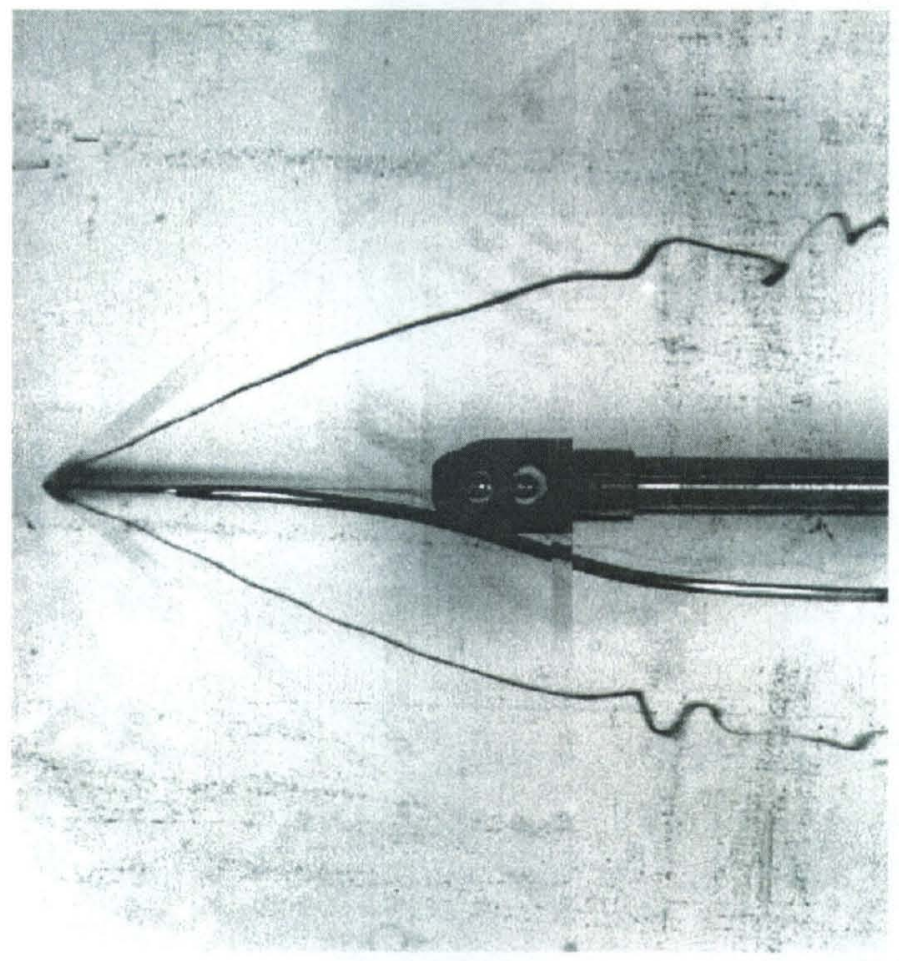

Figure 4.10. $\alpha=5^{\circ}$, planform view

For the $5^{\circ}$ angle of attack case (Figure 4.9 and Figure 4.10), the LEVs are seen to bend slightly inboard in going downstream; that is, their trajectory is not strictly linear, even if one were to ignore minor waviness. Departure from straight trajectories emanating from the wing apex is indicative of departure from a strictly conical velocity field.

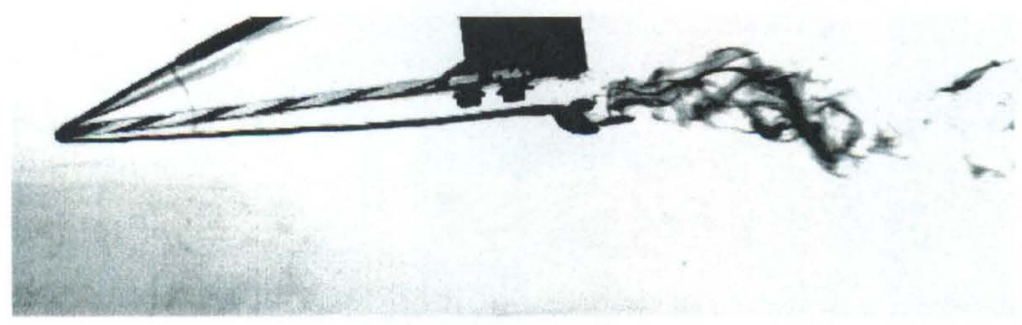

Figure 4.11. $\alpha=7.5^{\circ}$, side view 


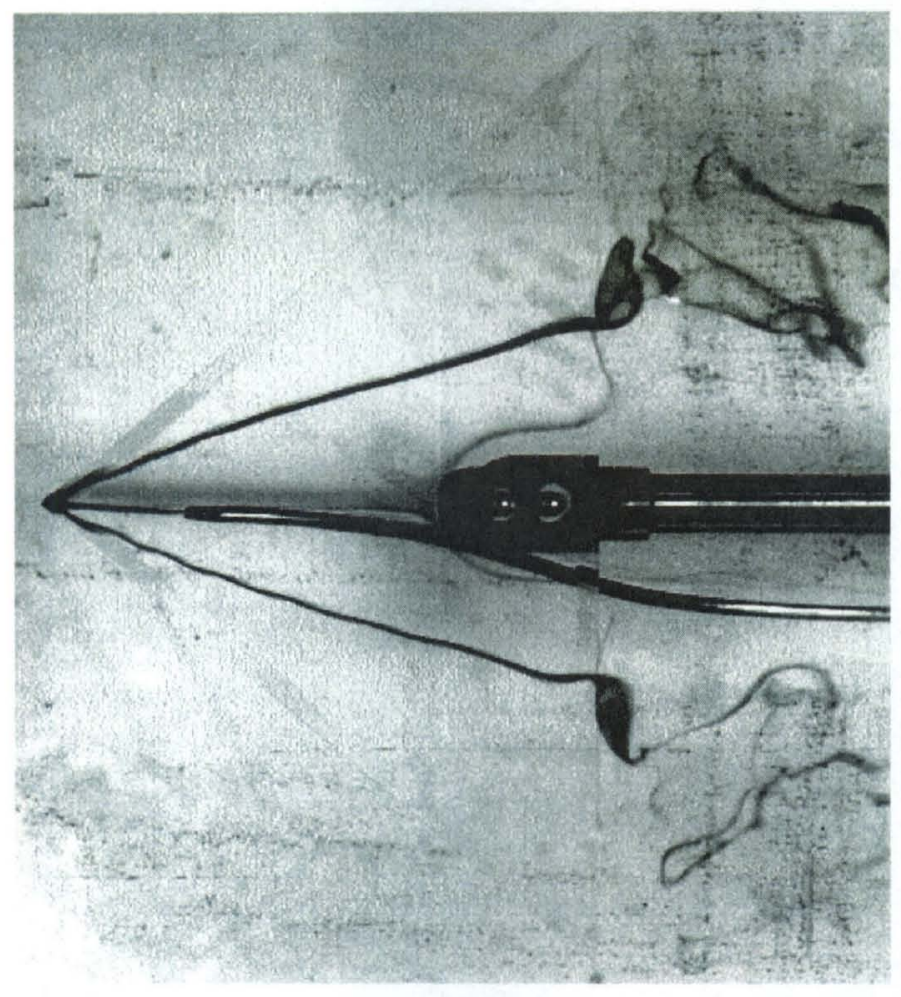

Figure 4.12. $\alpha=7.5^{\circ}$, planform view

At the $7.5^{\circ}$ angle of attack, the straight line LEV trajectories return. VB is seen to be approximately at the wing trailing edge, with considerable left/right symmetry. The actual geometry of the VB regions appears to differ from the usual case; the breakdown bubbles bend toward the trailing edge, apparently under the influence of the trailing edge potential flow effects.

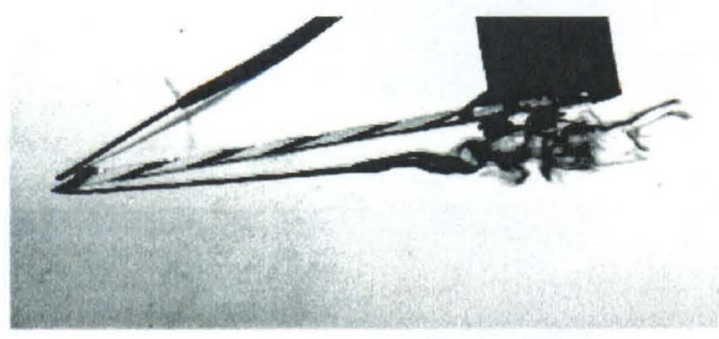

Figure 4.13. $\alpha=10^{\circ}$, side view 


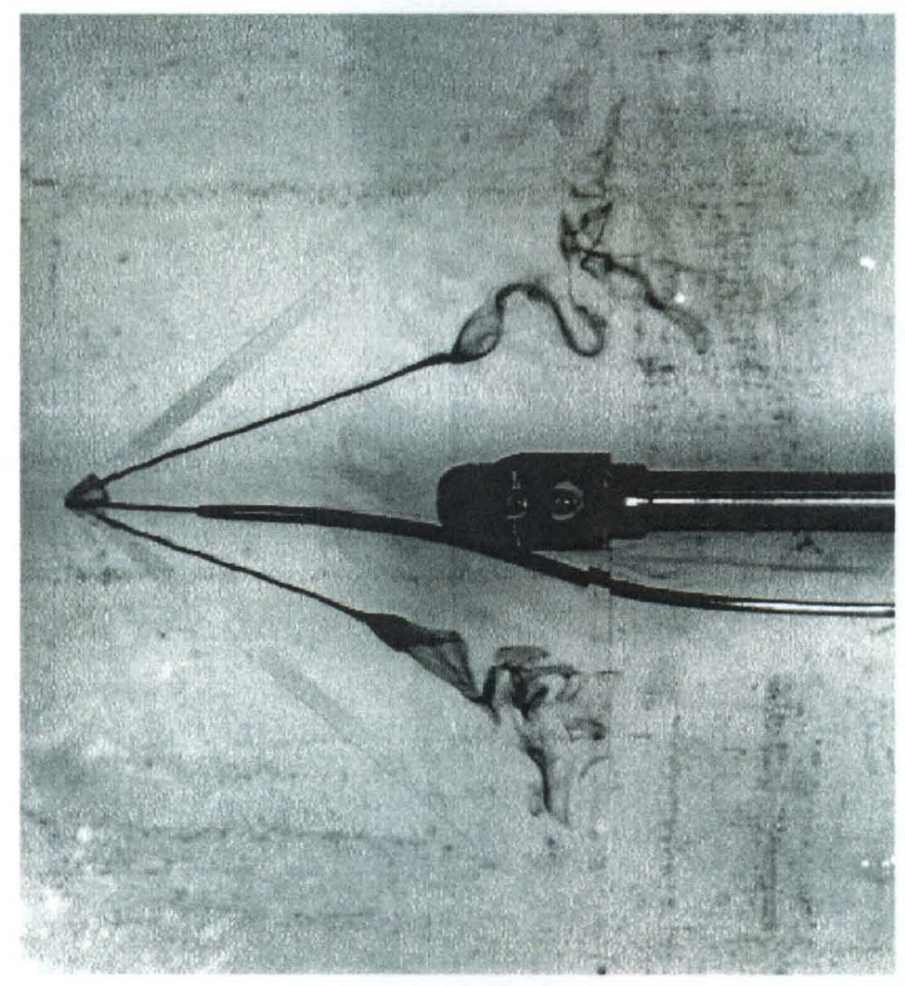

Figure 4.14. $\alpha=10^{\circ}$, planform view

At $\alpha=10^{\circ}$, vortex breakdown begins to take on its "usual" character; an identifiable zone of flow retardation and dye streak broadening is observed. In the above figure, "bubble" breakdown was observed, though the left and right LEVs had somewhat differently shaped bubbles. At lower angles of attack, "breakdown" was exhibited more as a poorly defined kinking of the dye streak. In Figure 4.14, the left VB region has a strong concentration of dye at the bubble front, indicative of stagnation. The right-side region is less concentrated, and is en route to transition to a spiral-type breakdown. Interestingly, for the $50^{\circ}$ wing, the bubble-type of breakdown was more common than the spiral-type. For the $65^{\circ}$ wing, the situation was precisely the reverse.

At angles of attack beyond $10^{\circ}$, the LEVs are appreciably less stable. 


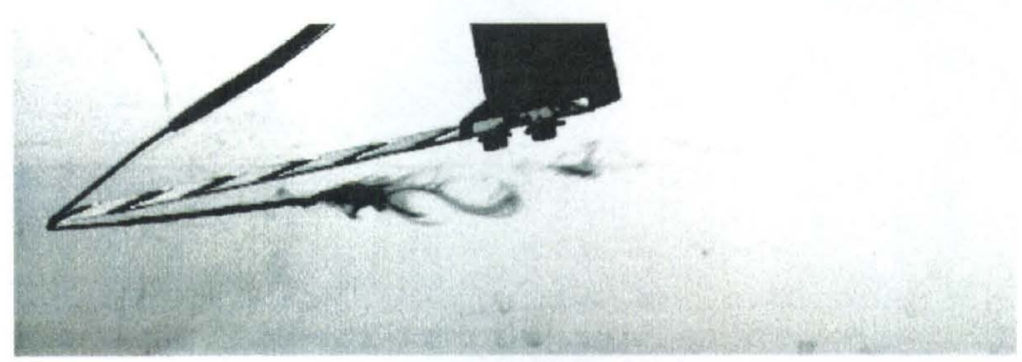

Figure 4.15. $\alpha=12.5^{\circ}$, side view

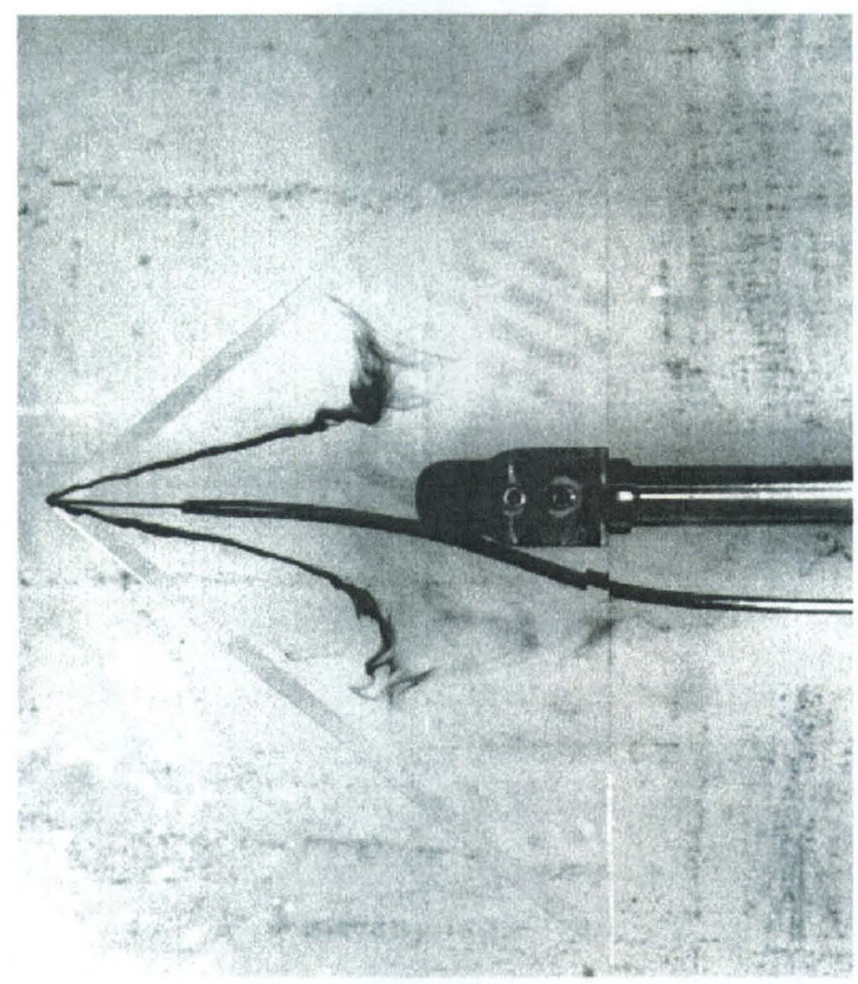

Figure 4.16. $\alpha=12.5^{\circ}$, planform view

At $12.5^{\circ}$, breakdown in the classical slender delta wing sense is again apparently gone, being replaced by a flow reversal distinct from the usual bubble or spiral. This is necessarily a transient process, since the situation in Figure 4.16 offers no "sink" to maintain a steady dye flow once the LEV trajectory reaches its turning points. While difficult to interpret, it appears that the VB mechanism in Figure 4.16 is neither spiral nor bubble, but of a third type. 


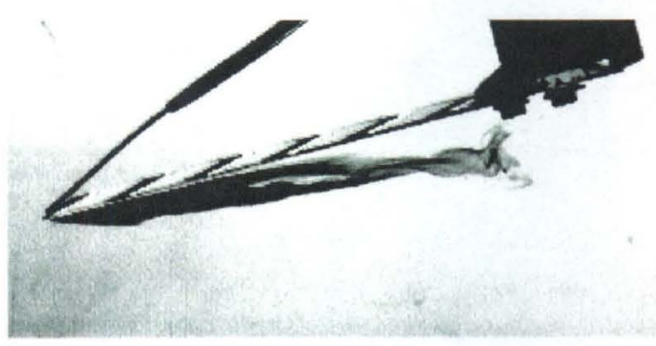

Figure 4.17. $\alpha=15^{\circ}$, side view

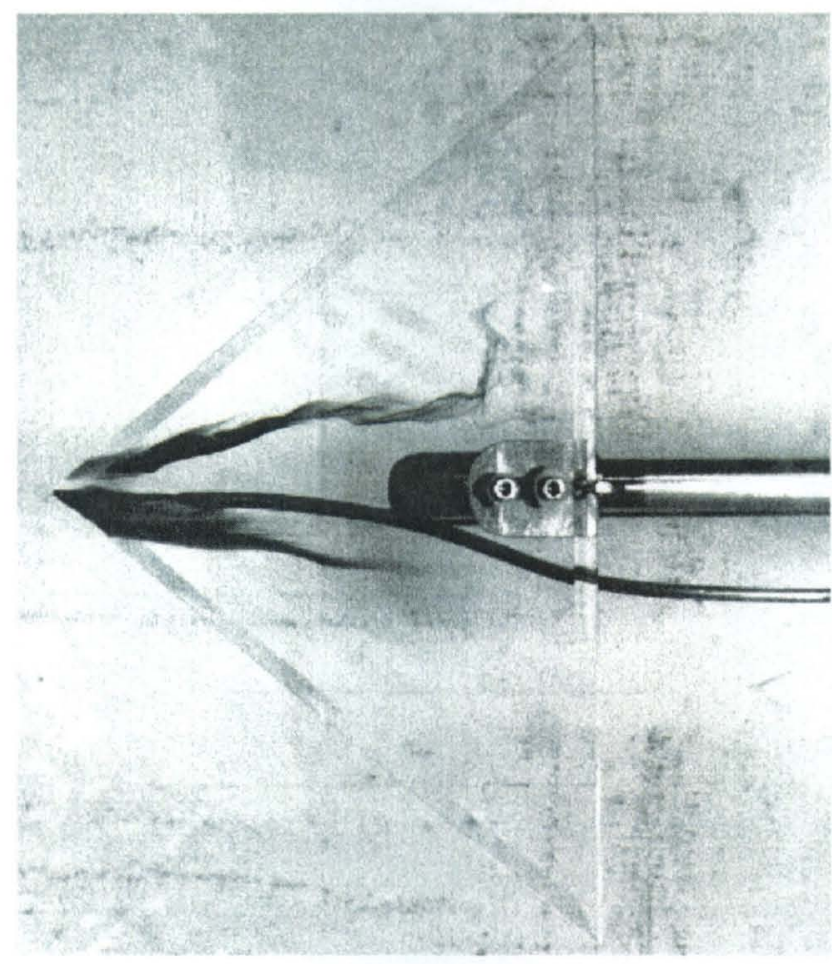

Figure 4.18. $\alpha=15^{\circ}$, planform view

At $15^{\circ}$ (Figure 4.17 and Figure 4.18 ), symmetric LEVs were very difficult to maintain. For $17.5^{\circ}$ and above, "coherent" symmetric LEVs were not observed. At $20^{\circ}$ (Figure 4.19 and Figure 4.20), the situation is one of "cloud" separation. 


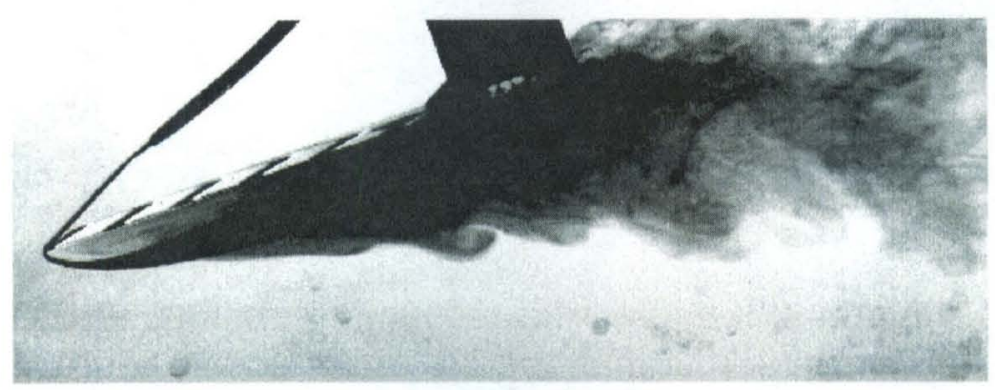

Figure 4.19. $\alpha=20^{\circ}$, side view

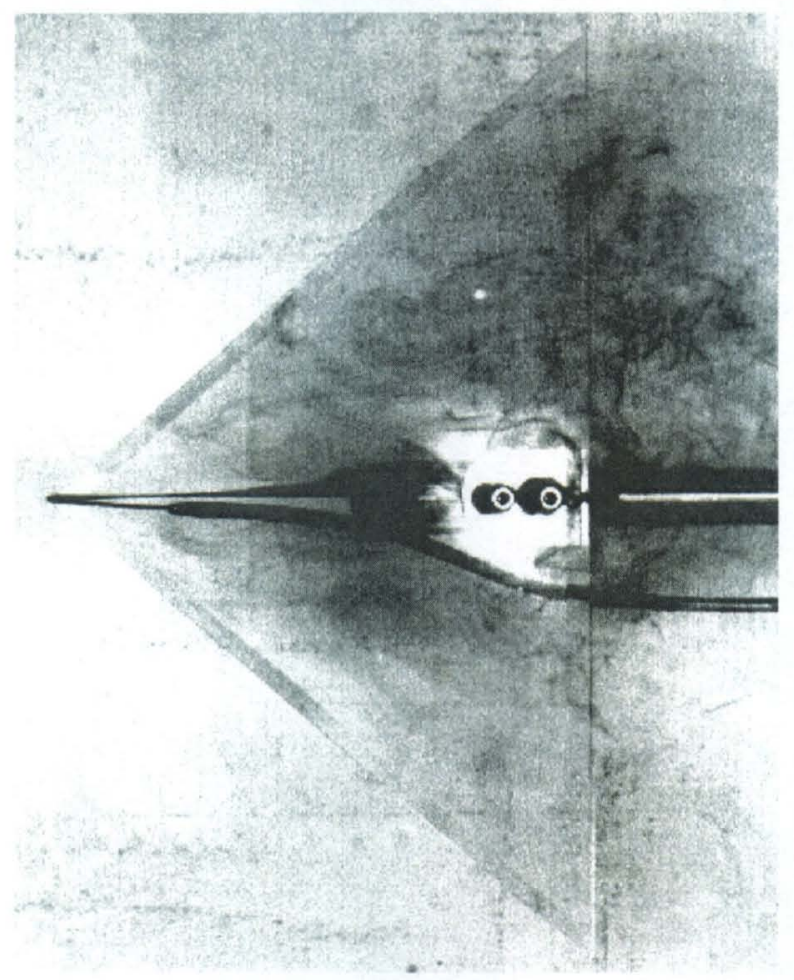

Figure 4.20. $\alpha=20^{\circ}$, planform view

In the side view (Figure 4.19) we see what look like Kelvin-Helmholz waves bounding the separated leeward region of flow from the free stream. This is qualitatively indicative of a complete loss of coherent lift-generating vortical activity, or in other words, amounts to "bluff body" separation. However, there is no evidence of bulk vortex shedding from the leading edges, as is generally the case for bluff bodies. 


\subsubsection{Secondary LEVs}

Secondary vortices, formed by interaction of the primary vortices and the leeward boundary layer, are most apparent at moderate angles of attack, $5^{\circ}-10^{\circ}$. In the following figures, the starboard-side primary and secondary vortex pair are shown. Waviness in the dye streak is again attributed to off-axis entrainment of the dye into the vortex paths.

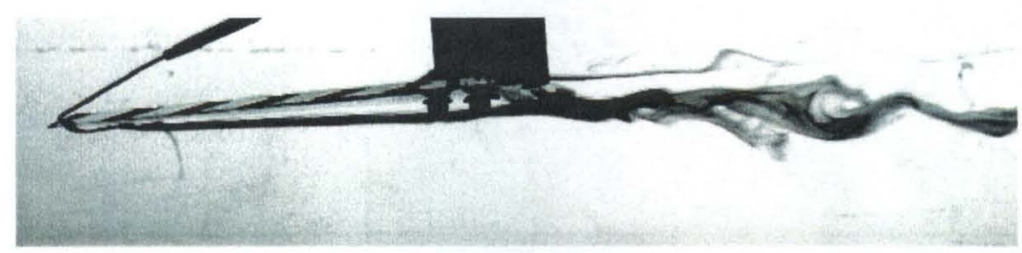

Figure 4.21. $\alpha=5^{\circ}$, side view

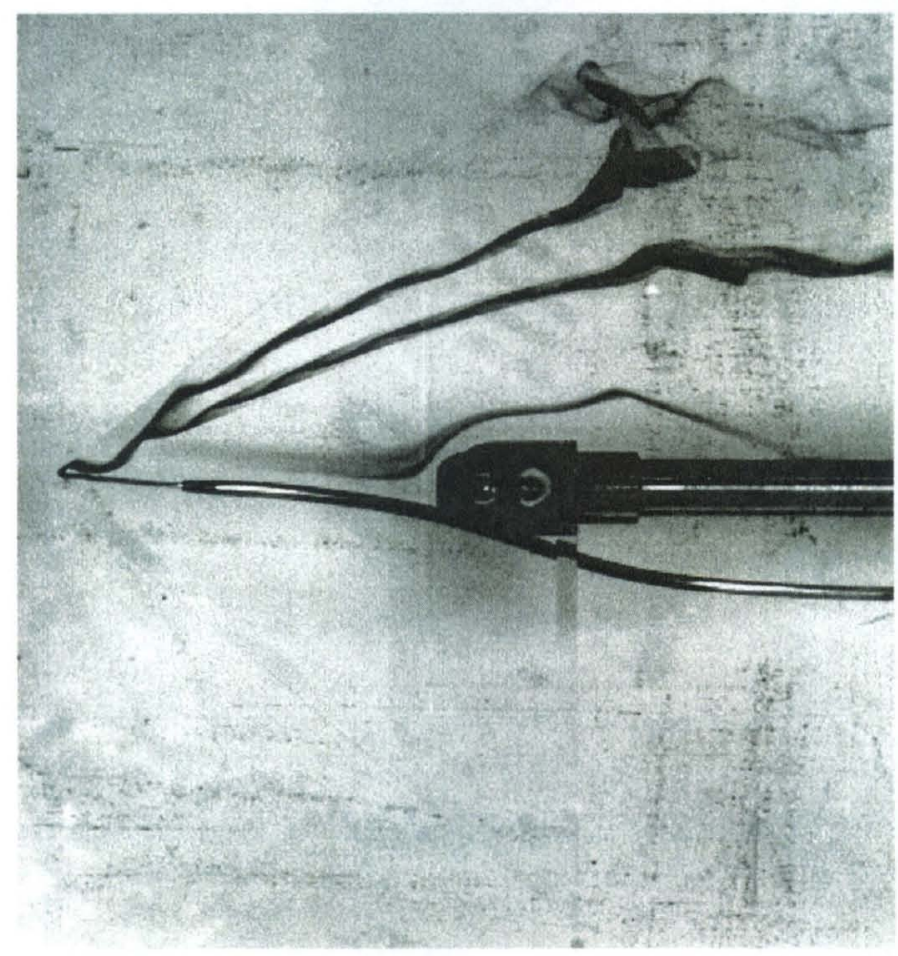

Figure 4.22. $\alpha=5^{\circ}$, planform view

In Figure 4.22, the secondary vortex (the upper dye streakline) is almost of the same extent as the primary. The former terminates in a "rotating" region just aft of the 
trailing edge, while the primary vortex continues in a weaker form further downstream (compare Figure 4.10). The primary vortex is again seen to bend slightly away from a straight trajectory. Thus, it is seen that modification of the dye injection scheme for purposes of observing the secondary vortex has not appreciably affected the primary vortex.

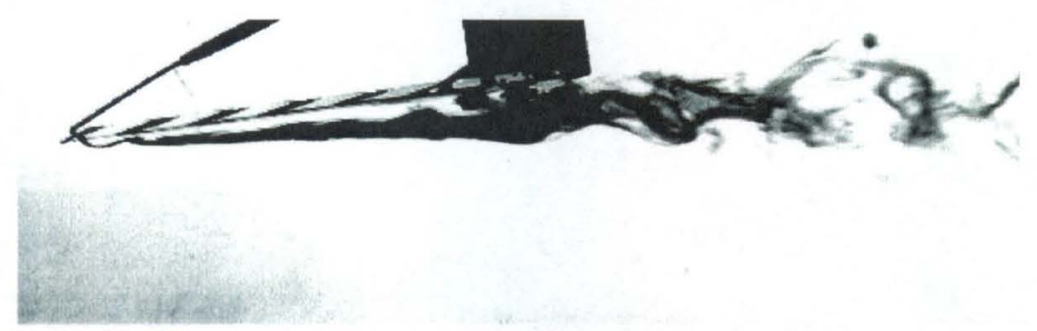

Figure 4.23. $\alpha=7.5^{\circ}$, side view

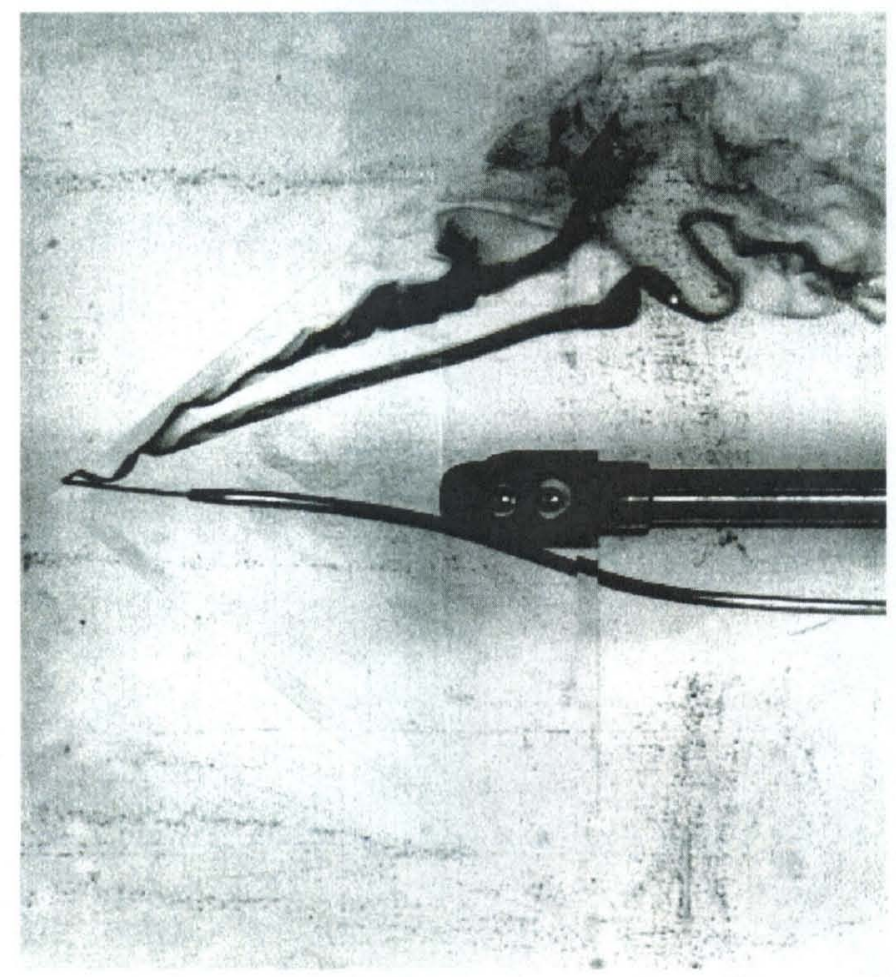

Figure 4.24. $\alpha=7.5^{\circ}$, planform view

At $7.5^{\circ}$, the secondary vortex has a markedly serrated shape. Its termination in a "rotating region" is broader and extends further upstream. 


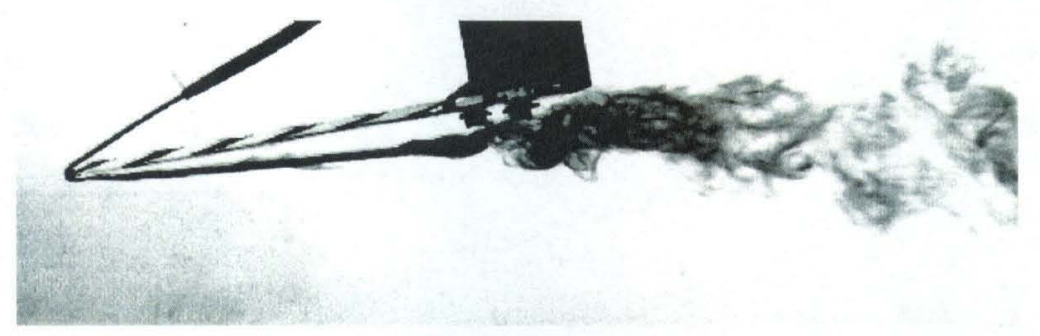

Figure 4.25. $\alpha=10^{\circ}$, side view

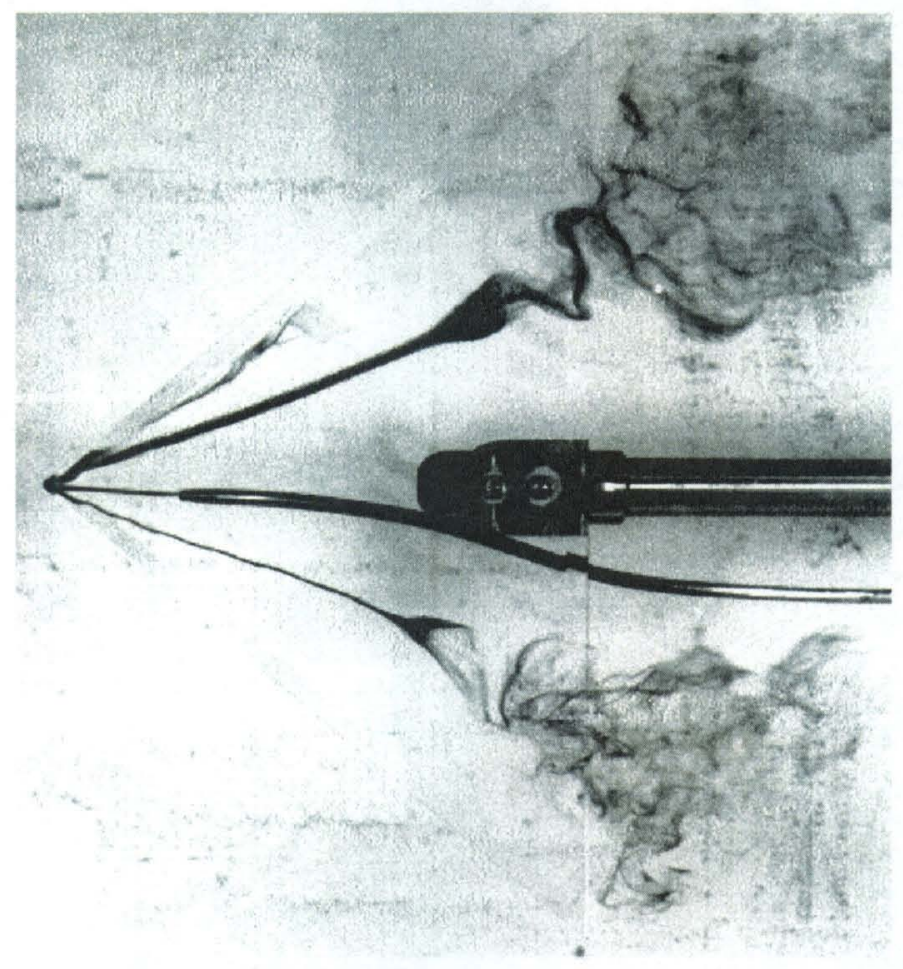

Figure 4.26. $\alpha=10^{\circ}$, planform view

The $\alpha=10^{\circ}$ case (Figure 4.26) is a limiting case. The left and right LEVs are clearly visible. Breakdown occurs at around two-thirds of the main chord. The secondary vortex is now very weak; indeed, it requires some faith to suppose that the vortex is still present. So, the secondary vortex is seen to disintegrate before the primary vortex. Again, neither the LEV trajectory nor the VB point is noticeably affected by the 
relative amount of dye, which is a reassuring affirmation of the robustness of the injection technique.

At angles of attack of $12.5^{\circ}$ and beyond, secondary flow could no longer be visualized by dye injection near the apex.

\subsubsection{Evidence of unsteadiness upstream of breakdown}

The higher angle of attack cases display marked unsteadiness - that is, there is an apparent exchange of stability between the left and right primary LEVs. Unsteadiness downstream of the breakdown region, and in the vicinity of the VB region itself, has long been known. Here we investigate unsteadiness upstream of VB, with the observation that in the following cases - and only in the following cases - was such flow behavior observed. The $65^{\circ}$ wing has no such behavior at the angles of attack under consideration. Of course, at higher angles of attack, there is evidence that even quite slender wings have such LEV unsteadiness upstream of the VB point, en route to the complete flow separation at $90^{\circ}$ angle of attack (Ayoub and McLachlan ${ }^{25}$ ).

Among the angles of attack considered here, appreciable unsteadiness was seen in the range of $12.5^{\circ}-17.5^{\circ}$ for the $50^{\circ}$ wing, though some variation in the VB location is already displayed at $10^{\circ}$. At $20^{\circ}$, there was not enough recognizable LEV structure to consider unsteadiness to be relevant, beyond fluctuations in the shear layer bounding the leeward separation region (Figure 4.19).

Data are nondimensionalized with respect to convective time, defined as

$$
t^{*}=c / U_{\infty}
$$


which for the $50^{\circ}$ wing computes to 1.34 seconds, or $\sim 40$ video frames. Each of the following series is started from nominal time zero. The question of periodicity in the destruction and reformation of coherent LEVs, or the upstream and downstream procession of the left and right $\mathrm{VB}$, is intriguing but largely unresolved, due in part to the very long data records necessary to capture such a slowly evolving motion. Some qualitative observations are given below. Frame time, $t$, is given in multiples of $t^{*}$

\subsubsection{1 $50^{\circ}$ wing, $\alpha=12.5^{\circ}$ series}

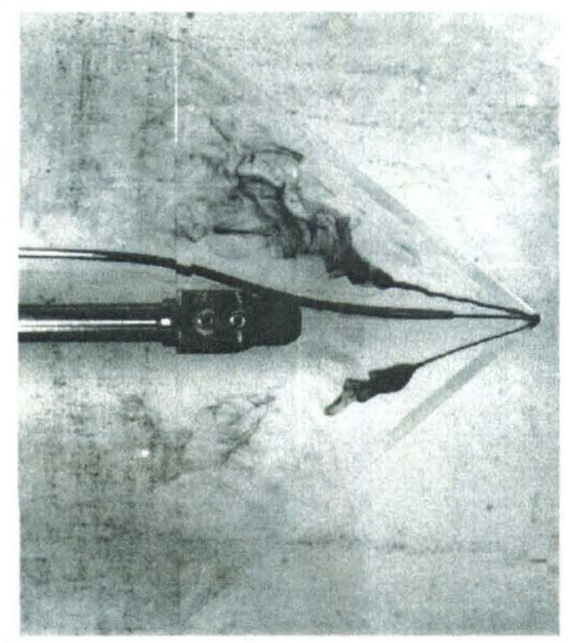

Figure 4.27. $t=0$

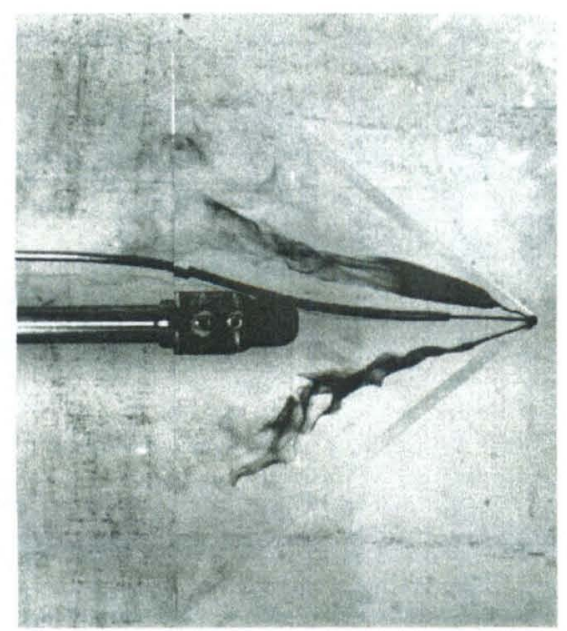

Figure 4.29. $t=4.0$

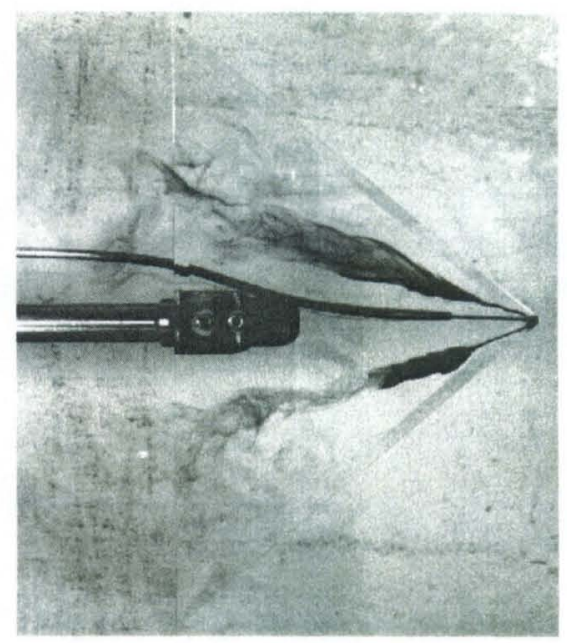

Figure 4.28. $t=2.74$

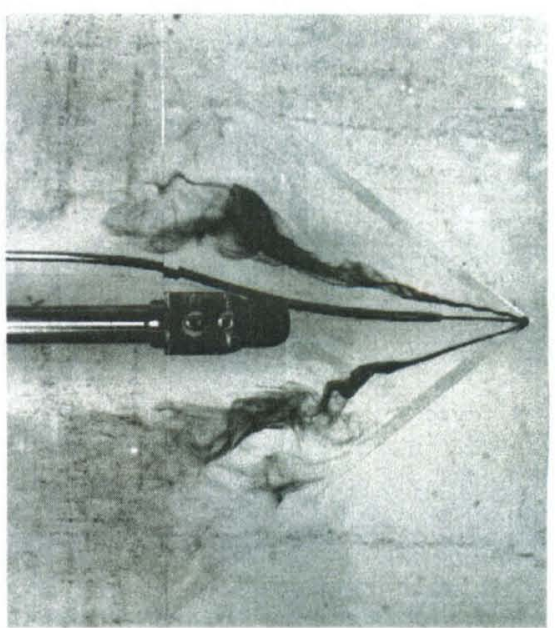

Figure 4.30. $t=5.81$ 


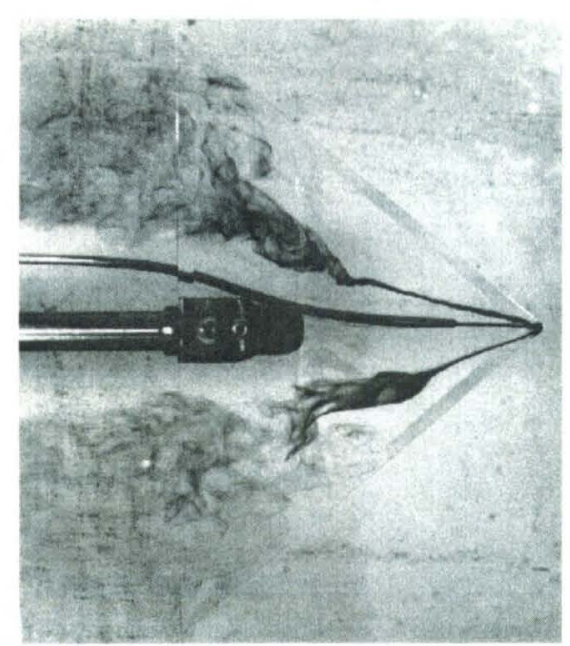

Figure 4.31. $t=7.33$

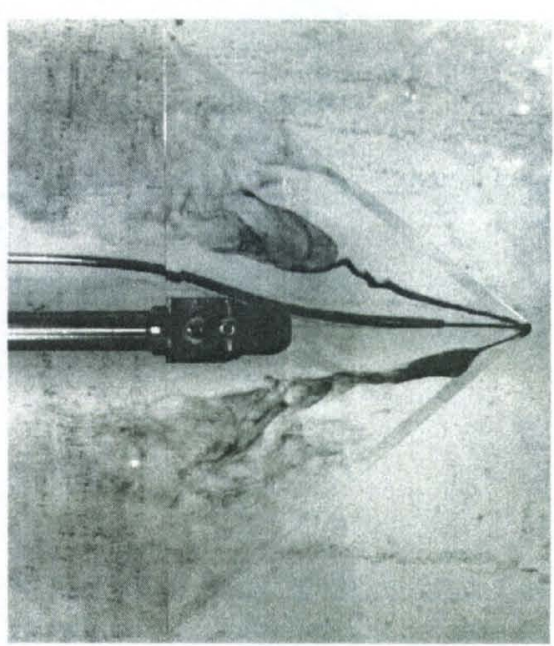

Figure 4.32. $t=8.90$

In going from $t=0$ to $t=8.9$, the behavior of the LEVs can be described as follows: a bubble-like VB region on the starboard side forms and moves upstream, while on the port side, an elongated bubble forms by coalescence of a wavy (but not spiral) breakdown structure. This breakdown structure is a version of the type shown in Figure 4.16. By $t=3$, the port structure overtakes the starboard structure in its upstream movement. The starboard LEV then reforms, dissipating its VB bubble and replacing it with a wavy-type breakdown much further downstream, while the port-side elongated bubble lingers near the apex. By $t=6$ the port-side bubble also collapses to a tight vortex core, and the starboard-side VB region again coalesces to a bubble. This bubble then resumes its upstream journey, while the port-side VB region retreats further downstream and takes on a more typical spiral shape.

Figure 4.27 and Figure 4.31 have similar LEV structures, breakdown locations, and post-breakdown dye signatures. Very casually, the "periodicity" at $12.5^{\circ}$ angle of attack then appears to be of about 7 convective times. A second sequence of images for 
the same test conditions is presented next, showing a yet greater variety of possible LEV shapes.

\subsubsection{2 $50^{\circ}$ wing, $\alpha=12.5^{\circ}$ second series}

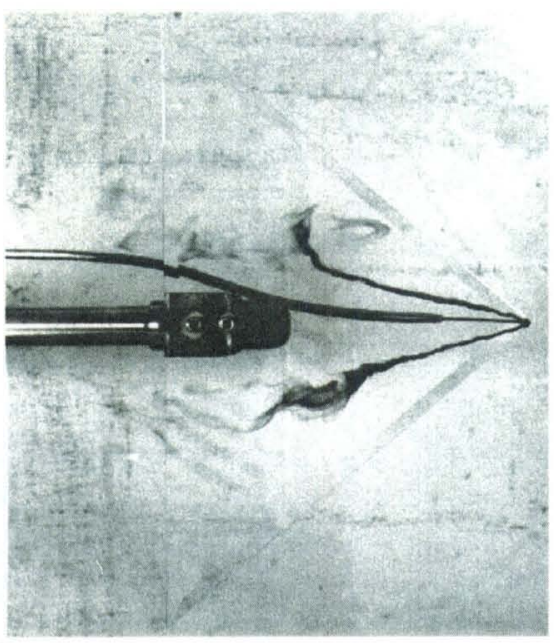

Figure 4.33. $t=0$

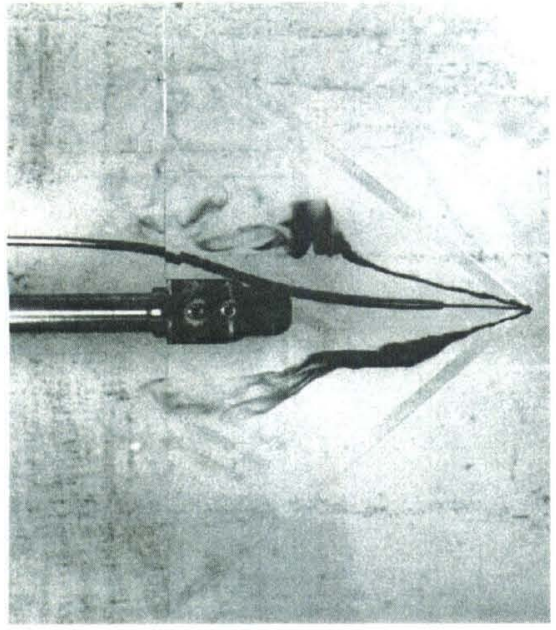

Figure 4.35. $t=2.57$

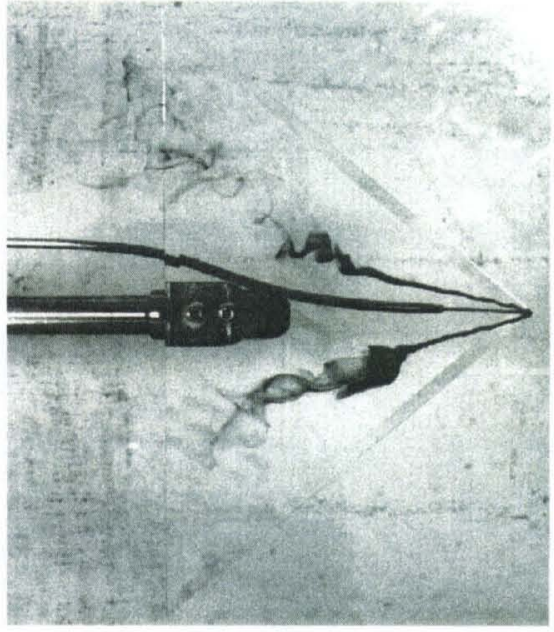

Figure 4.34. $t=1.32$

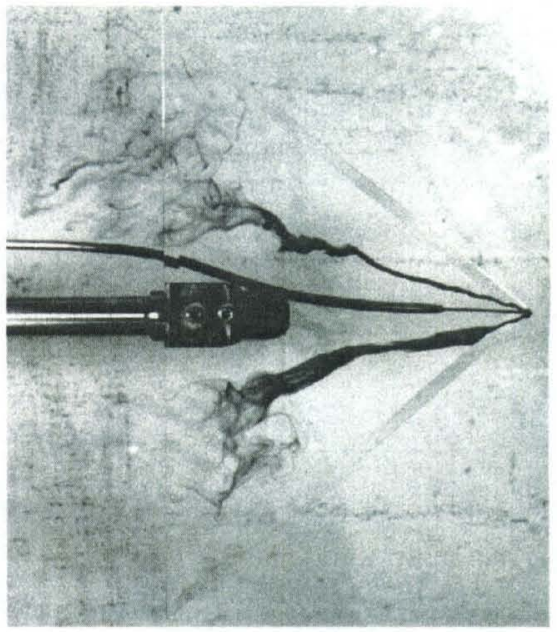

Figure 4.36. $t=3.57$ 


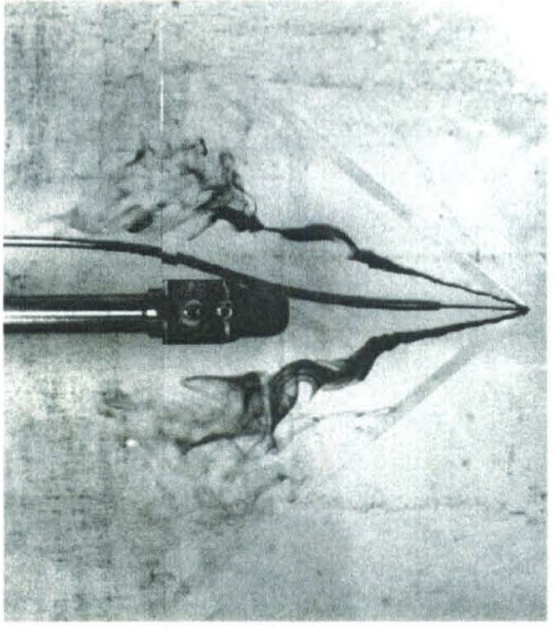

Figure 4.37. $t=4.57$

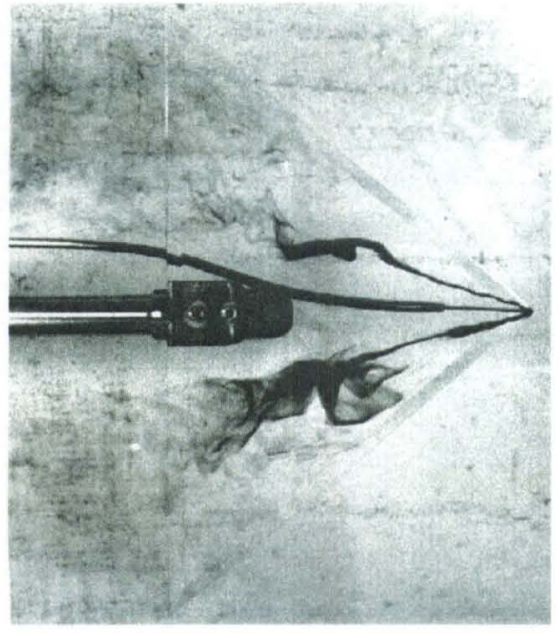

Figure 4.39. $t=6.48$

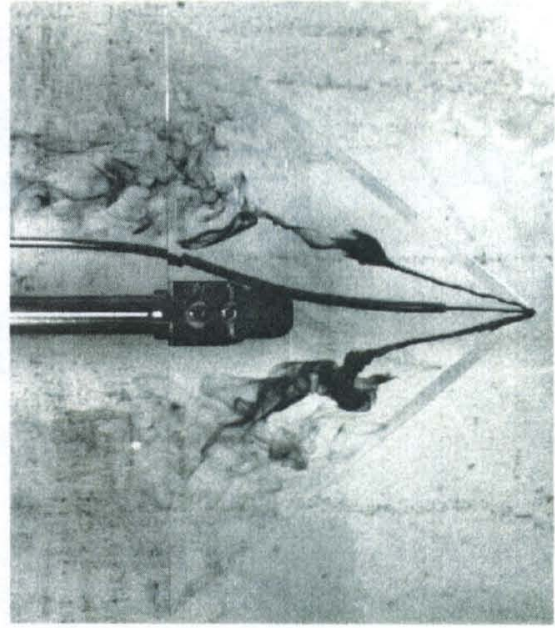

Figure 4.38. $t=5.43$

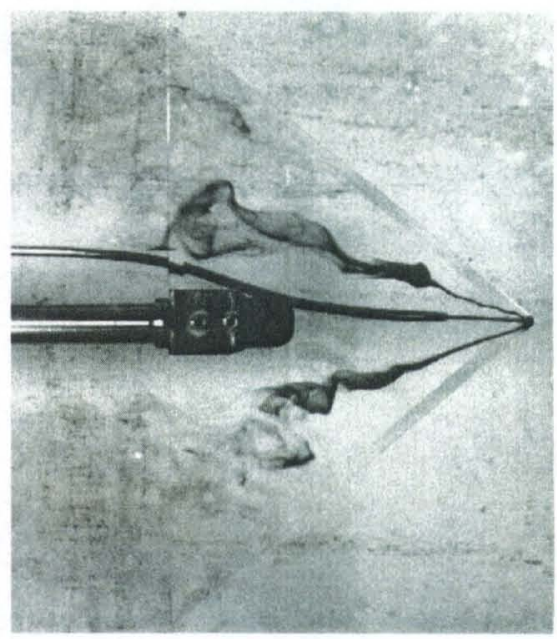

Figure 4.40. $t=7.80$

At $t=0$, the dye appears to illustrate a breakdown region forming in the starboard LEV. In the next image, the starboard VB region expands and moves upstream, then stretches and thins out in the next two images. By $t=4.5$, both LEVs are undergoing what appears to be a spiral VB. The port-side LEV then transitions to bubble-type burst, whereas the starboard LEV appears to terminate in a "whorl"-type pattern, with flow reversal in going outboard. In the next two convective times, the port-side bubble disintegrates as the VB region is stretched in the streamwise direction, but then reforms, 
and proceeds upstream. Meanwhile the starboard-side whorl collapses to a spiral-shaped VB region.

\subsubsection{50 ${ }^{\circ}$ wing, $\alpha=15^{\circ}$ series}

At $15^{\circ}$ angle of attack, the vortex cores are much less prominent. Evidently, ever weaker radial pressure gradients are entraining less and less dye.

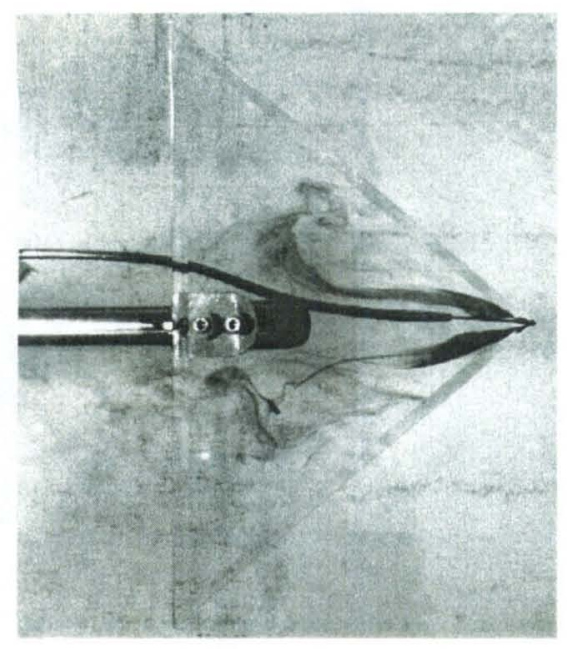

Figure 4.41. $t=0$

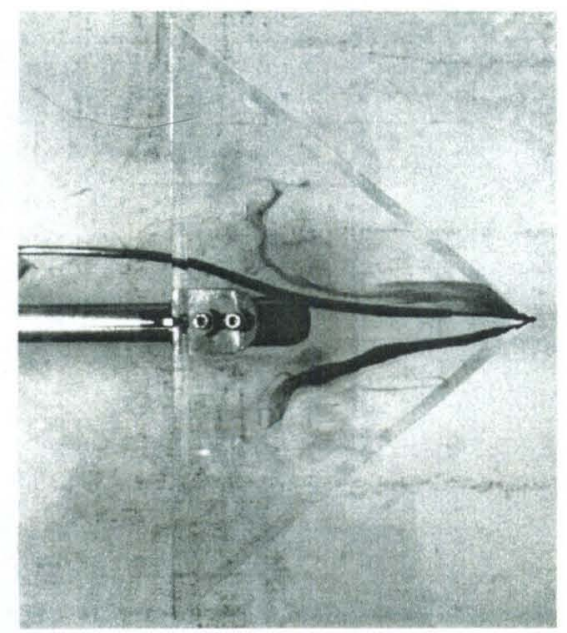

Figure 4.43. $t=2.29$

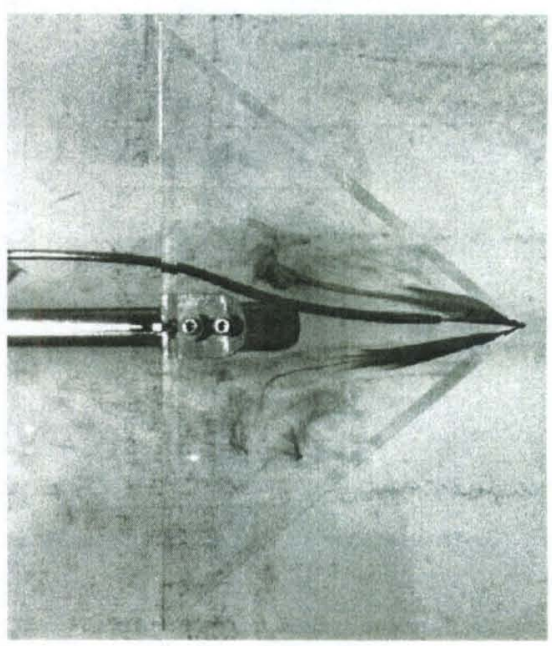

Figure 4.42. $t=1.05$

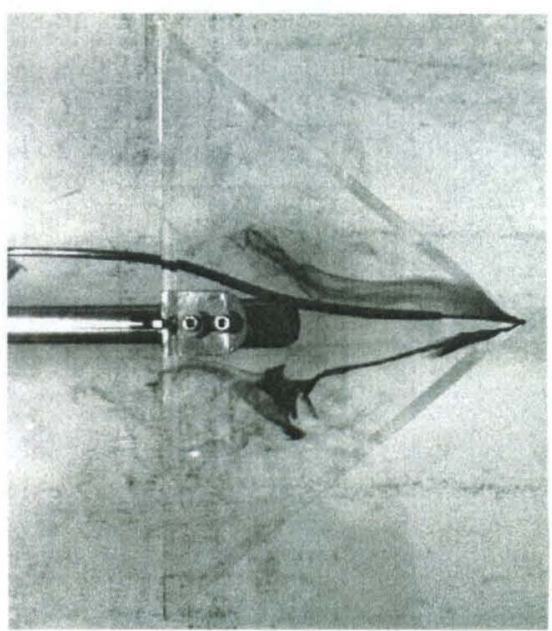

Figure 4.44. $t=4.0$ 


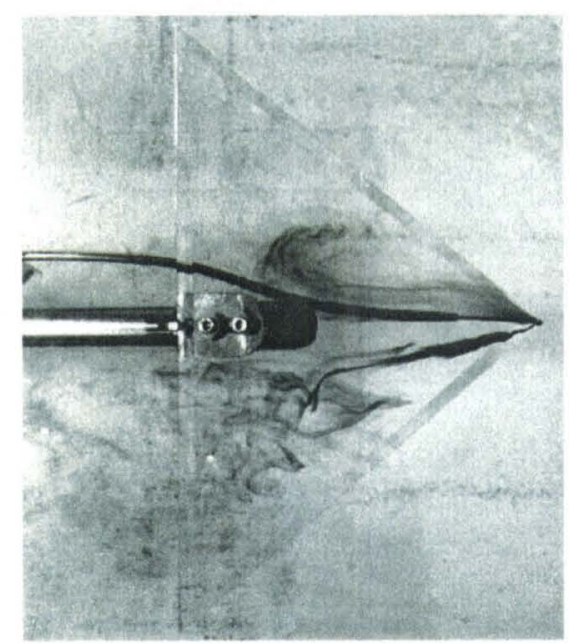

Figure 4.45. $t=5.86$

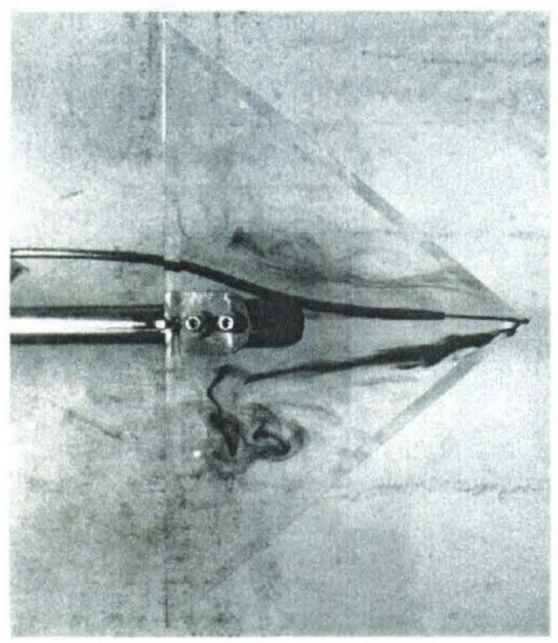

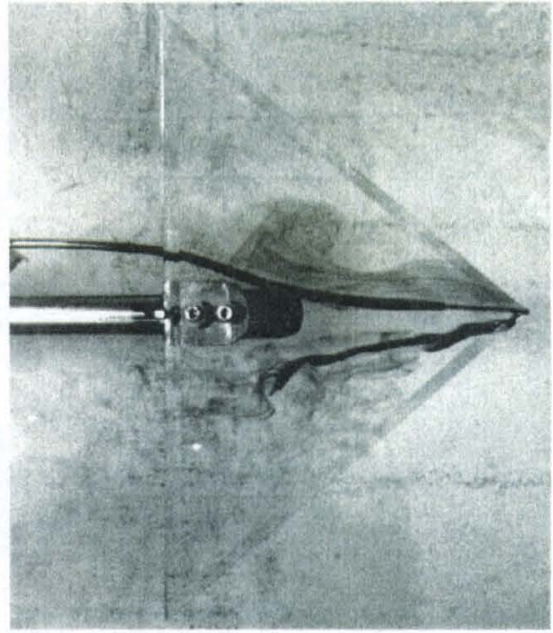

Figure 4.46. $t=7.40$

\section{Figure 4.47. $t=9.57$}

Figures $5.41,5.44$, and 5.47 are vaguely similar, so in this case, the characteristic period can be regarded as $\sim 5$ convective times. The whorl pattern terminating a LEV is more prevalent than in the $12.5^{\circ}$ angle of attack case.

\subsubsection{4 $50^{\circ}$ wing, $\alpha=17.5^{\circ}$ series}




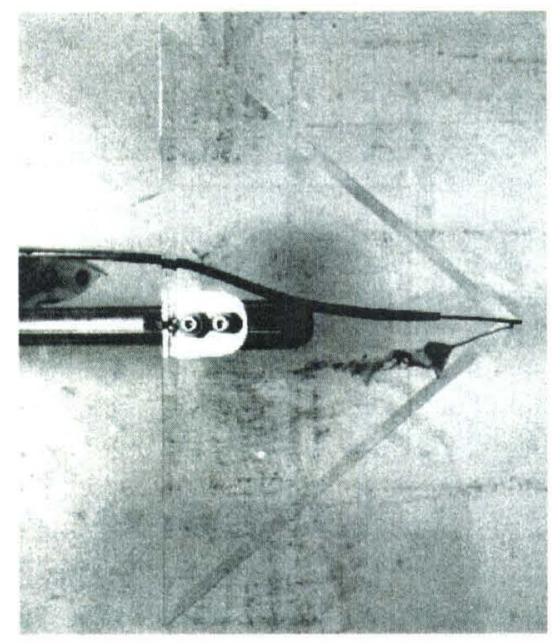

Figure 4.48. $t=0$

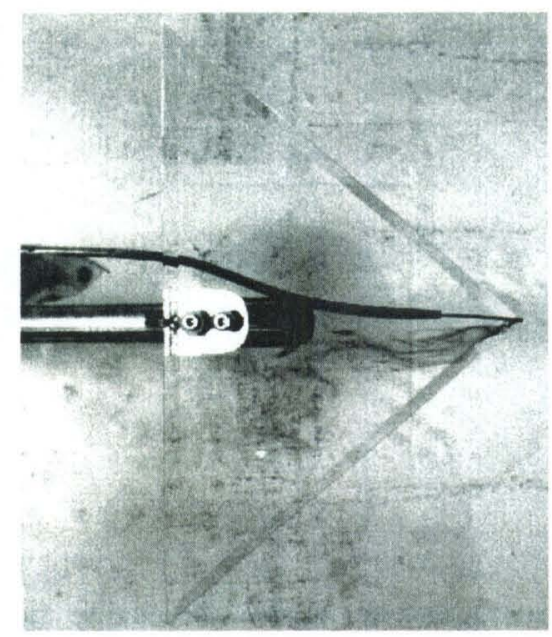

Figure 4.50. $t=3.69$

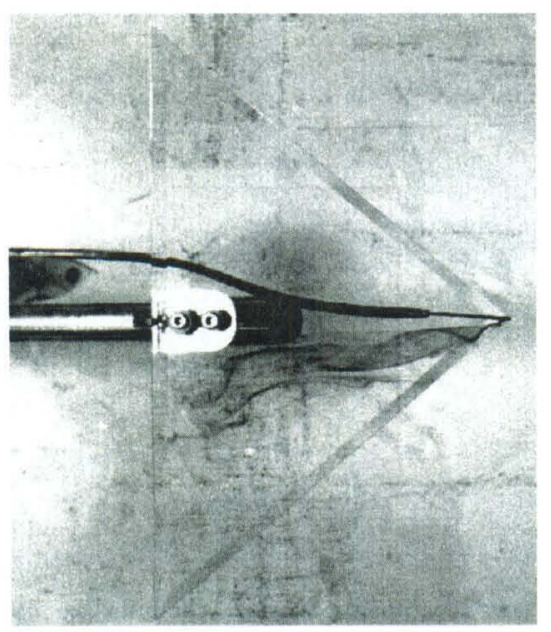

Figure 4.52. $t=7.45$

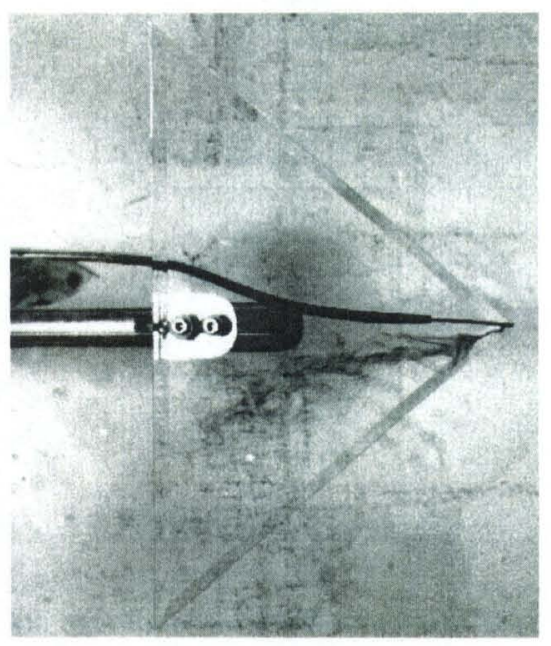

Figure 4.49. $t=2.27$

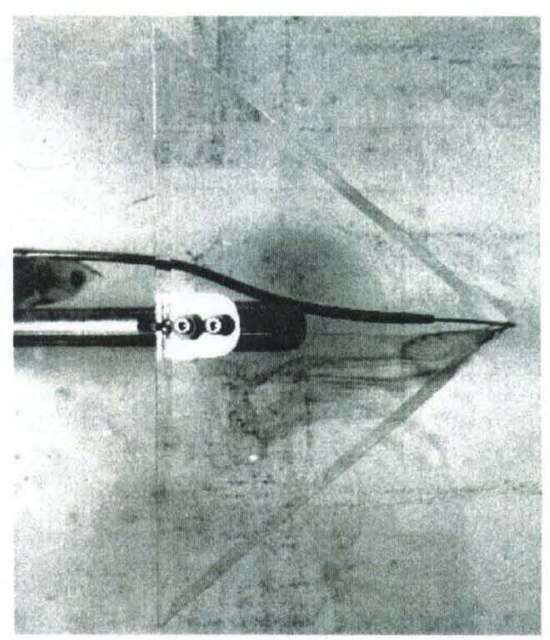

Figure 4.51. $t=4.38$

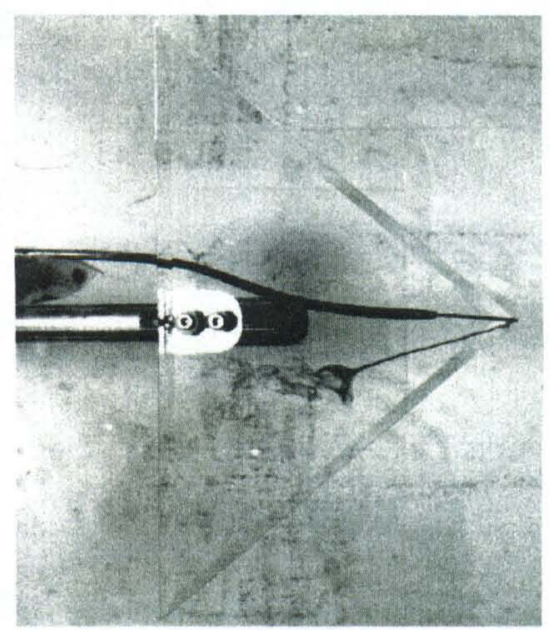

Figure 4.53. $t=9.84$ 


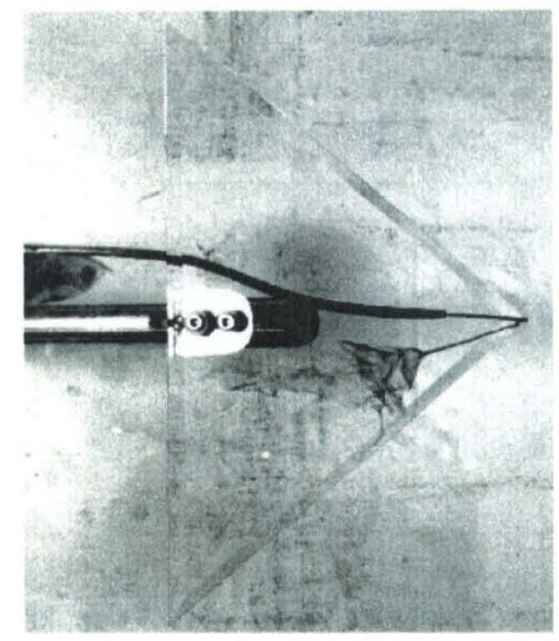

Figure 4.54. $t=12.2$

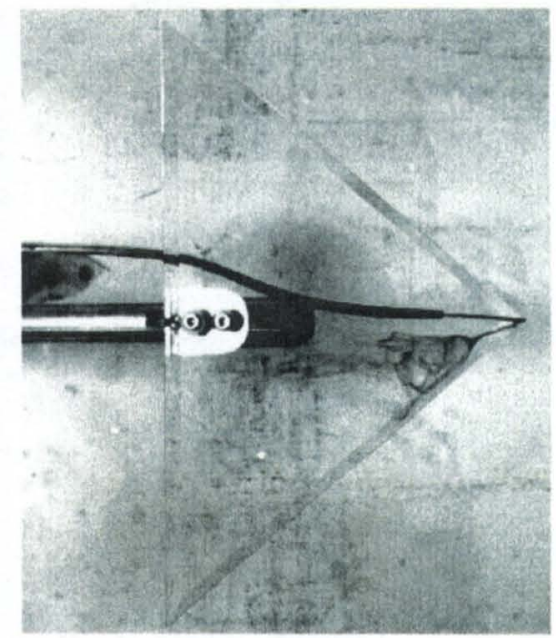

Figure 4.55. $t=13.7$

Figures 5.48 and 5.93 share the property of a well-defined starboard LEV and no port LEV at all, suggesting a periodicity of $\sim 9$ convective times. Again, the temporal length of the data record is insufficient to make a claim of periodicity with any semblance of rigor. To observe an LEV, the dye injection probe was biased toward the starboard side in this data series. It is seen that the VB location moves from roughly $\mathrm{z} / \mathrm{c}=0.5$ all the way to the apex, in which case the vortex is replaced by a weak spiral motion. Spiral VB per se was not observed, and in those cases where a definitive breakdown was observed downstream of a coherent vortex, that breakdown was of the bubble form.

\subsubsection{Motivations for PIV}

The flow visualization images of Section 4.4.3 are qualitative illustrations of substantial unsteadiness not only in the breakdown location, but of LEV structure upstream of breakdown. The presence of such unsteadiness motivates the application of field-type velocimetry techniques, as being superior to pointwise techniques. The large scale of unsteady motions relaxes requirements for especially high accuracy of 
measurement at any given point in the flow, further motivating the use of PIV-based techniques. However, the fully three-dimensional nature of the temporal evolution of the flow makes the application of a 2-D technique difficult, since there is no obvious choice of "preferred" orientation for the light sheet and interrogation region. This is in marked distinction with the steady case, especially for slender wings, where as already mentioned, the streamwise gradients are much smaller than the crossflow-plane gradients, and the choice of light sheet orientation is clearer. So it is particularly in the nonslender, unsteady cases that stereoscopic PIV (or perhaps, some other 3-D or quasi-3-D variant) is preferable.

\subsection{Breakdown locations}

By visual inspection of the pixel coordinates corresponding to the location where the dye streaks first depart from a nominally straight and organized motion, the location of VB can be roughly determined. Tracking the left and right LEVs, the observed breakdown locations are summarized in the following figures.

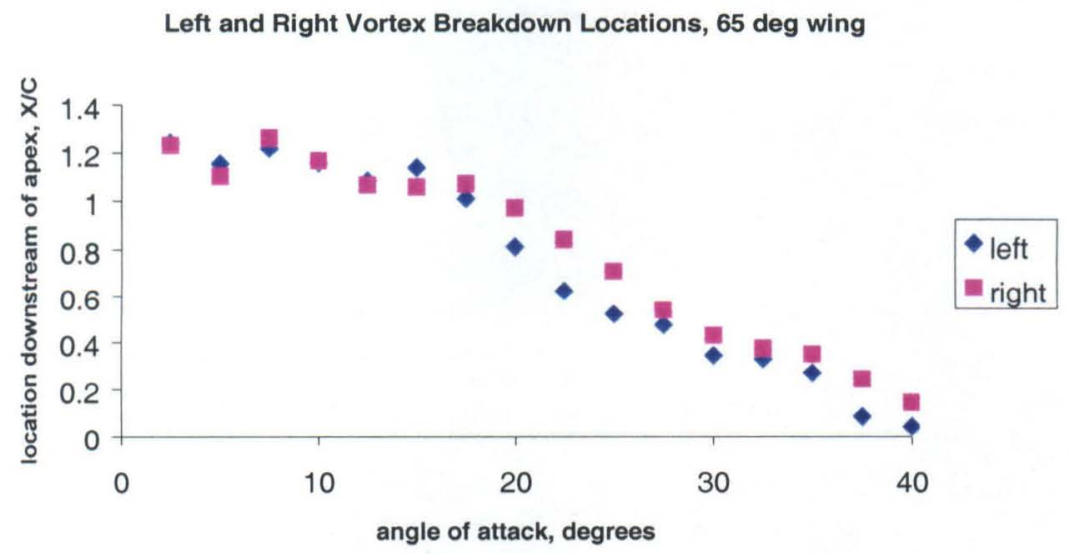

Figure 4.56. Breakdown downstream locations, $65^{\circ}$ wing 
Data for the $65^{\circ}$ in the literature are quite extensive. For instance, comparison to the data compiled by Jobe ${ }^{16}$, Figure 1.4, indicates that breakdown in the present experiment is somewhat downstream of the general trends in the literature. For instance, at $20^{\circ}$ angle of attack Figure 4.56 shows breakdown at $\mathrm{z} / \mathrm{c}=0.8$, whereas the average in Figure 1.4 is around 0.7. However, the large scatter in Figure 1.4 indicates that the present data are quite reasonable.

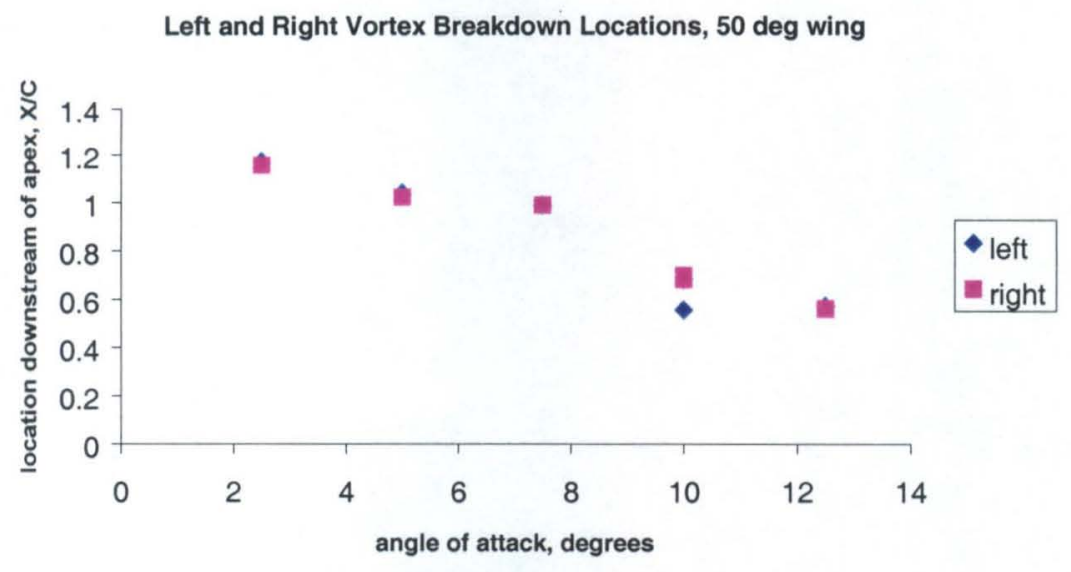

Figure 4.57. Breakdown downstream locations, $50^{\circ}$ wing

Asymmetry in the left and right LEVs in Figure 4.56 can be attributed to minor misalignment of the wing in yaw or roll.

In Figure 4.57, the plotted breakdown locations are a rough estimate of the mean of the aforementioned unsteady VB locations for conditions such as those at at $12.5^{\circ}$ angle of attack.

In those cases where breakdown occurs aft of the trailing edge, breakdown location is taken to be at the "kink" in the dye streak, where the dye trajectory follows the free stream direction instead of fanning out conically from the wing apex, as seen in the planform view. "Proper" breakdown was observed to occur some distance downstream 
of this "kink" - often one or two root chord lengths downstream of the trailing edge. Indeed, the notion of breakdown occurring at some definable distance aft of the trailing edge raises the interesting question of the role of adverse pressure gradient. An adverse pressure gradient is frequently invoked as the root cause of breakdown over the wing planform. But aft of the trailing edge, there is essentially zero adverse streamwise pressure gradient, and hence, one needs to consider another explanation for vortex breakdown or change of direction of the dye streak.

For the $50^{\circ}$ wing, the literature is much more sparse. Wentz and Kohlman ${ }^{18}$ considered a $50^{\circ}$ wing, but their data set begins at $15^{\circ}$. Nelson and Pelletier ${ }^{68}$ took flow visualization data for a $50^{\circ}$ wing at $\operatorname{Re}=50000$, for angles of attack from $7^{\circ}$ to $18^{\circ}$. At $7^{\circ}$, VB was observed to lie at approximately $z / c=0.75$. VB reached the apex by $18^{\circ}$. The upstream progression with increasing angle of attack was close to linear. There was no evidence of the unsteadiness observed in the present experiments in the $12^{\circ}-20^{\circ}$ range. Conceivably, this unsteadiness is related to velocity fluctuations reported by Klein et al. ${ }^{69}$ for a wing of moderate sweep, but those fluctuations were of well-defined frequency content, unlike those observed in the present flow visualizations. Also, the present data shows a further downstream location of VB for the lower angles of attack, but an earlier onset of complete stall of the wing.

Streamwise excursions of VB location are by no means unique to delta wings of moderate sweep, though in the case of wings of larger sweep, the excursions are of much lower extent. For example, the flow visualization data of Truneva ${ }^{70}$ revealed streamwise VB excursions of about $10 \%$ of root chord, for a wing of $60^{\circ}$ sweep at a Reynolds number of $\sim 2500$. 


\subsection{Core axial speed measurements}

Since the dye stream approximately follows the trajectories of the centerlines of the leading edge vortices, motions of the dye can be interpreted as convection in the LEV cores. Thus, by turning the dye on and off with the video cameras running, movement of the dye front from frame to frame gives a measure of the local axial velocity in the vortex core. One can thus obtain an estimate for the LEV core axial velocity. The approach is similar to the more detailed analysis of Lambourne and $\mathrm{Bryer}^{71}$, also for a $65^{\circ}$ wing.

LEV core velocities are plotted for the $65^{\circ}$ and $50^{\circ}$ wings in Figure 4.58 and Figure 4.59 , respectively, normalized by the free stream velocity. Here and elsewhere, no attempt has been made to distinguish between the axial component of the velocity in the core, and the magnitude of the full velocity vector. The distinction was deemed to be modest, since for both wings the "sweep" of the dye trajectories is small, and the axial component of velocity dominates the other (Cartesian) components.

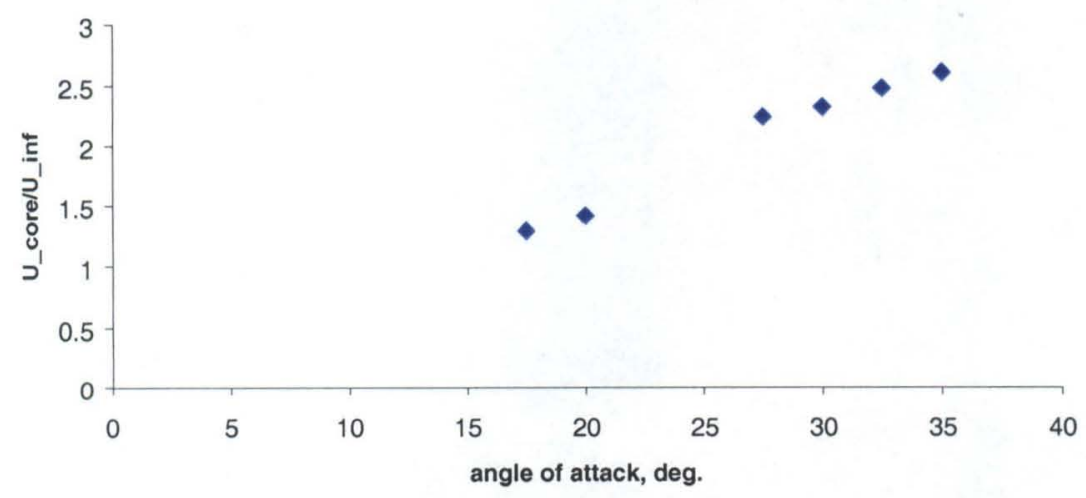

Figure 4.58. Normalized axial velocity in core, $65^{\circ}$ wing 


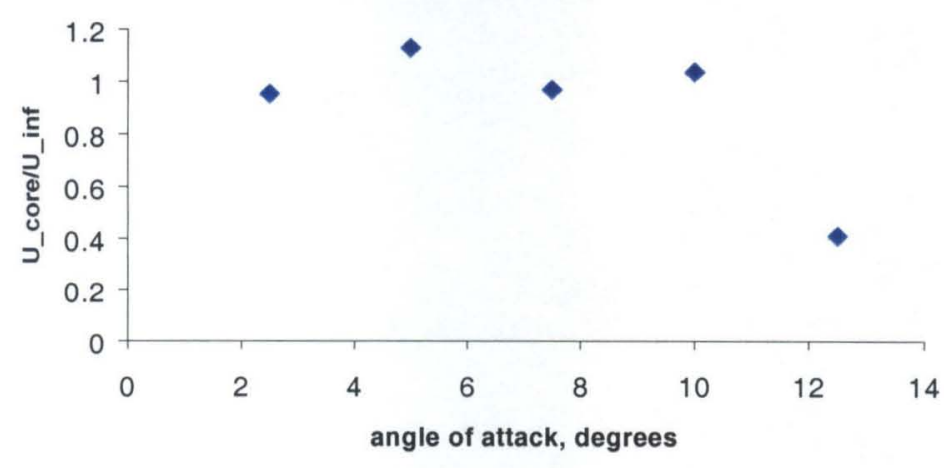

Figure 4.59. Normalized axial velocity in core, $50^{\circ}$ wing

It should be emphasized that these data are taken on the assumption that core velocity is constant in going downstream; that is, the dye does not accelerate in going along its trajectory. Essentially, this is a conical flow assumption.

\subsection{Summary, and comments on the distinction between the $65^{\circ}$ and $50^{\circ}$ wings}

Apart from the obvious fact of more rapid onset of breakdown, the flowfield of the $50^{\circ}$ wing differs from that of the $65^{\circ}$ wing in several important ways; three are discussed below.

The first is the greater visible presence of secondary separation for the $50^{\circ}$ wing, as revealed by thick, bright dye streaks at moderate angle of attack. This is in some sense to be expected, since the LEV trajectories for the lower sweep wing are closer to the wing surface, and therefore LEV/boundary layer interactions - which ultimately are responsible for secondary separation - can be expected to be stronger. At higher angles of attack, the secondary separation is weaker, at least in part because the LEVs themselves are further from the wing leeward surface, and the consequent boundary layer interaction is weaker. 
The second distinction is the presence of unsteadiness. From $\alpha=12.5^{\circ}$ until the full stall angle, the left and right vortex breakdown points alternate in streamwise extent, one being considerably further downstream than the other, and subsequently vice versa. No systematic periodic behavior was observed, although a nominally "chaotic" behavior is not necessarily the case, either. Occasionally, past the breakdown point the dye following the LEV would curve outboard toward where the secondary separation region would have been, which evidently is a region of stagnation and even reverse flow.

Thirdly, and perhaps most importantly, the variation in LEV core axial velocity with increasing angle of attack is quite different for the two wings. Observed behavior for the $65^{\circ}$ wing is consistent with the classical results for slender wings at high angle of attack; that is, the core speed is on the order of 2-3 times the free stream speed at angles of attack of $20^{\circ}$ and above. At lower angles of attack, the core speed is slower. The variation with increasing angle of attack is nominally linear. On the other hand, the core speed for the $50^{\circ} \mathrm{LEVs}$ is very close to free stream speed for those angles of attack where the LEVs are steady and coherent (and where, perhaps not coincidentally, secondary vortices are very strong), but drops off to below free stream speed as the LEVs disintegrate. This is reminiscent of the transition from "jet-like" to "wake-like" axial velocity profile in going from a pre-breakdown to post-breakdown state of the LEVs. But as will be shown in Chapter 6, and as suggested from the flow visualization results in Section 4.4.1, this wake-like profile occurs at angles of attack well below those where vortex breakdown has already crossed the spanwise station of interest.

Whereas results for the $65^{\circ}$ wing were in complete accordance with expectations, the $50^{\circ}$ wing exhibited unusually strong leading edge vortices. One can speculate that 
this was due to the choice of leading edge bevel geometry, or to Reynolds number affects.

The flow visualization provides some interesting motivations for use of stereoscopic PIV. In particular, the $50^{\circ}$ wing flow regime in the $12-20^{\circ}$ angle of attack regime is marked by large-scale quasi-periodic unsteadiness. This would be missed by traverses with a single-point technique, or by 2 -D piv. The comparatively low core speed in the $50^{\circ}$ wing cores raises some interesting issues of the balance of vorticity production at the leading edges and downstream convection in the LEV cores. This can be further elucidated with stereo PIV measurements. 


\section{Chapter 5 SDPIV Data in Crossflow Planes}

This chapter presents a resume of stereoscopic digital particle velocimetry data obtained in the present study. The test matrix and various ancillary tasks are reviewed, and velocity vector data are presented in various projections. In all cases, the domain is still planar, but the data have three components, making the choice of presentation format a matter of some finesse. Two-dimensional projections of streamlines are given.

Subsequently, vorticity computed from the velocity is plotted. While in general only the out-of-plane vorticity component is given, all three components are presented in selected cases.

\subsection{The test matrix}

\subsubsection{Schedule of angle of attack sweeps}

Stereo PIV tests were scheduled to overlap with the flow visualization tests. However, data were taken only at the lower angles of attack, covering the range over which the $50^{\circ}$ transitioned to stall. In the following table , " $\mathrm{X}$ " indicates a single planar slice of data, and "XX" indicates a 3-planar set, taking advantage of the 3-component vorticity formulation of Section 2.11 .

Table 5-1 . Stereo PIV test matrix; angle of attack sweep

\begin{tabular}{|c|c|c|}
\hline $\begin{array}{c}\text { angle of } \\
\text { attack, deg }\end{array}$ & Wing & Wegree \\
\hline
\end{tabular}

\begin{tabular}{|c|c|c|}
\hline 5 & $\mathrm{X}$ & $\mathrm{X}$ \\
\hline 7.5 & $\mathrm{X}$ & $\mathrm{X}$ \\
\hline 10 & $\mathrm{XX}$ & $\mathrm{X}$ \\
\hline 12.5 & $\mathrm{X}$ & $\mathrm{X}$ \\
\hline 15 & $\mathrm{XX}$ & $\mathrm{XX}$ \\
\hline & & \\
\hline 20 & $\mathrm{X}$ & $\mathrm{X}$ \\
\hline
\end{tabular}


For each angle of attack, data were taken in crossflow planes (that is, normal to the free stream, rather than normal to the wing planform), at downstream stations separated by $0.25 "(6.35 \mathrm{~mm})$. A data set corresponding to one crossflow plane at one angle of attack, for each wing, will be referred to as a data record. It was found that for stations upstream of about 0.5 " $(12.7 \mathrm{~mm})$ behind the model apex, the salient flow features were too small to adequately resolve with the available camera lenses, particle size, and particle seeding. Meanwhile, the furthest downstream stations were limited by the available field of view; the LEV cores in going further downstream would exceed the image bounds, requiring different lenses and a resetting of the entire optical setup. That was deemed intractable for the present experiment.

Most data records consisted of 200 frames for each of the two cameras for every plane of interrogation, at the usual NTSC rate of 30 frames/second. For the $50^{\circ}$ wing (107 $\mathrm{mm}$ root chord), 200 frames corresponds to a nondimensional testing time of 5.0 for $80 \mathrm{~mm} / \mathrm{s}$ free stream speed. For the $65^{\circ}$ wing (193 mm root chord), the nondimensional testing time is then 2.8. Data records for the $50^{\circ}$ wing, for $12.5^{\circ}$ angle of attack, were 500 frames long; thus a nondimensional time of 12.5. This was in an effort to capture the unsteadiness anticipated from the flow visualization experiments, for which this angle of attack was chosen as a representative case.

\subsubsection{Schedule of streamwise station sweeps}

When the streamwise locations are normalized by the model root chords, the data plane spacing becomes different for the two wings. This is illustrated in Table 5-2. Data for every entry in Table 5-2 were taken at the six angles of attack in Table 5-1. 


\section{Table 5-2. Stereo PIV test matrix, nondimensionalized locations of interrogation planes}

$\begin{array}{cc}\begin{array}{l}50 \text { degree wing } \\ \text { downstream }\end{array} & 65 \text { degree wing } \\ \text { location (normalized) } & \begin{array}{l}\text { downstream } \\ \text { location (normalized) }\end{array} \\ 0.118 & 0.066 \\ 0.178 & 0.099 \\ 0.237 & 0.132 \\ 0.296 & 0.165 \\ 0.355 & 0.197 \\ 0.414 & 0.230 \\ 0.473 & 0.263 \\ & 0.296 \\ & 0.329 \\ \end{array}$

Thus, for the $50^{\circ}$ wing there are 70 PIV data records, and for the $65^{\circ}$ wing there are 80. Of the former, 28 are triple-plane data records (for $10^{\circ}$ and $15^{\circ}$ angles of attack), and the rest are single-plane. Of the latter, 10 are triple-plane (only $15^{\circ}$ angle of attack). As mentioned previously, only the triple-plane data can be expected to render the out-ofplane velocity gradients, but they are tedious to acquire without some form of automation. Streamwise spacing between the data planes in a triplet was 0.050 " $(1.27 \mathrm{~mm})$ between every two planes, with observable uncertainty in measurement of the planar streamwise location of about $0.07 \mathrm{~mm}$, or $5 \%$.

It should be noted that the effective data plane streamwise location with respect to the wing planform will also slightly vary with angle of attack, since the horizontal projection of the wing planform contracts with increasing model incidence.

As a check on the effect of Reynolds number, the $65^{\circ}$ wing was also tested at $15^{\circ}$ angle of attack at a speed of $32 \mathrm{~mm} / \mathrm{s}$. This is just slightly above the observed minimal freestream speed at which the water tunnel is still stable (that limit being about $28 \mathrm{~mm} / \mathrm{s}$ ). 


\subsubsection{Orientation of imaging regions}

A "fully" 3-D data set can be approximated by assembly of planar slices of crossflow planes, in succeeding downstream stations. In practice, the utility of this approach is constrained by such factors as the increasing physical size of the LEV (and of the local wingspan) in going downstream, necessitating a reduction in optical magnification as seen by the cameras. This means either moving the cameras with respect to the light sheet, or changing lens focal length, or making some similar alteration to the optical setup. In all cases, the stereo PIV arrangement will require realignment and recalibration, which is a tedious task. For the data presented in Chapter 6, only one setting of the stereo PIV arrangement was used (Figure 4.4).

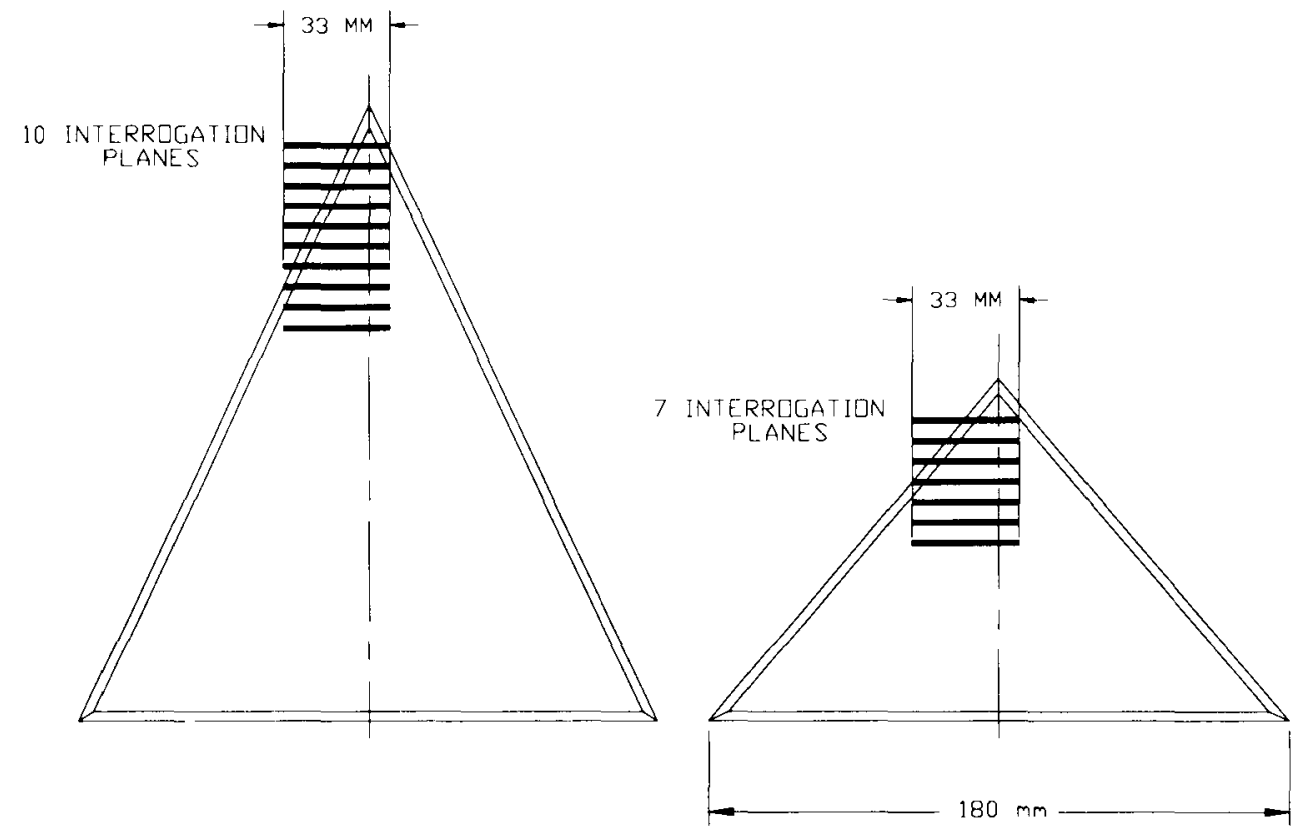

Figure 5.1. Arrangement of stereo PIV interrogation planes

\subsubsection{Relation to flow visualization}

From the flow visualization experiments, it is seen that especially for the $50^{\circ}$ wing, the dye trajectories have much less "sweep" than the leading edges (i.e., are more 
aligned with the freestream flow direction). This is important in scheduling the PIV tests in order to capture as much of one of the LEV cores at as high magnification as possible, and to march as far downstream as possible without realignment of the stereo PIV optics. Thus, the fact that for the $50^{\circ}$ wing, the vortex cores do not greatly move outboard in going downstream, allows the entire above-mentioned test matrix to be conducted for one setting of the optics. Unfortunately, the extent to which the secondary separation can be resolved is more limited, since the sweep of the secondary vortex core trajectories is greater than that of the primary. And the extent to which the origin of the LE separation can be resolved is yet more limited.

\subsection{Some procedural issues}

\subsubsection{Raw particle images}

A sample raw particle image is given in Figure 5.2. The delta wing model appears as a dark band near the top of the frame. As in the flow visualization, the wing is mounted upside down, with the leeward surface facing the bottom of the figure.

It appears that the presence of the wing does not cause a great optical disturbance in the particle images. However, accretion of particles on the model surface can not be entirely eliminated, even if the model is scrubbed between acquisition of each data record. The presence of these "stuck" particles appears in the processed velocity data as a spurious boundary layer. All though not so clear from the figure below, particle accretion is especially pronounced along lines of separation on the leeward surface. This of course is consistent with the basic principle of surface oil flow visualization of skin friction lines where oil accretes along separation lines. 


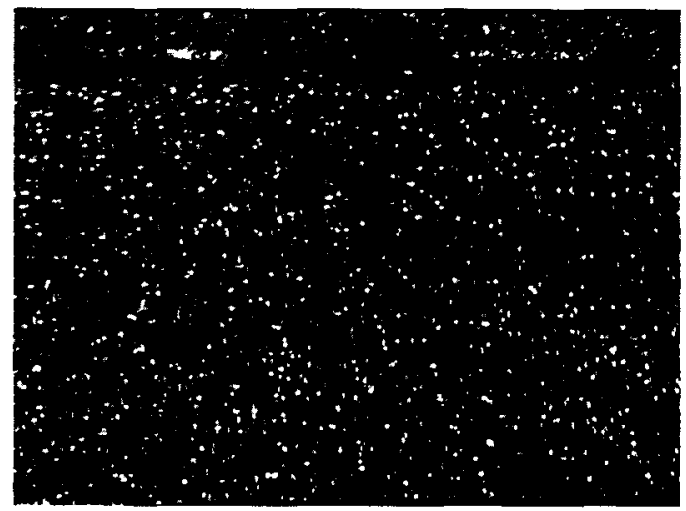

Figure 5.2. Typical raw particle field

\subsubsection{Model location}

If the model is left undisturbed in the test section for several hours, it accumulates a substantial amount of particles on its surface. This fact can be used to "locate" the model in the image frame. Left and right images of the wing are taken, and are then dewarped in the same manner as particle images. The following is a typical case, for the $65^{\circ}$ wing, at $5^{\circ}$ angle of attack. The cross-sectional plane is $2 "(50.8 \mathrm{~mm})$ downstream of the wing apex. The images have been reoriented, with the wing leeward surface facing up, and the right-side leading edge on the image right side. Thus the right-side LEV will appear over the wing.
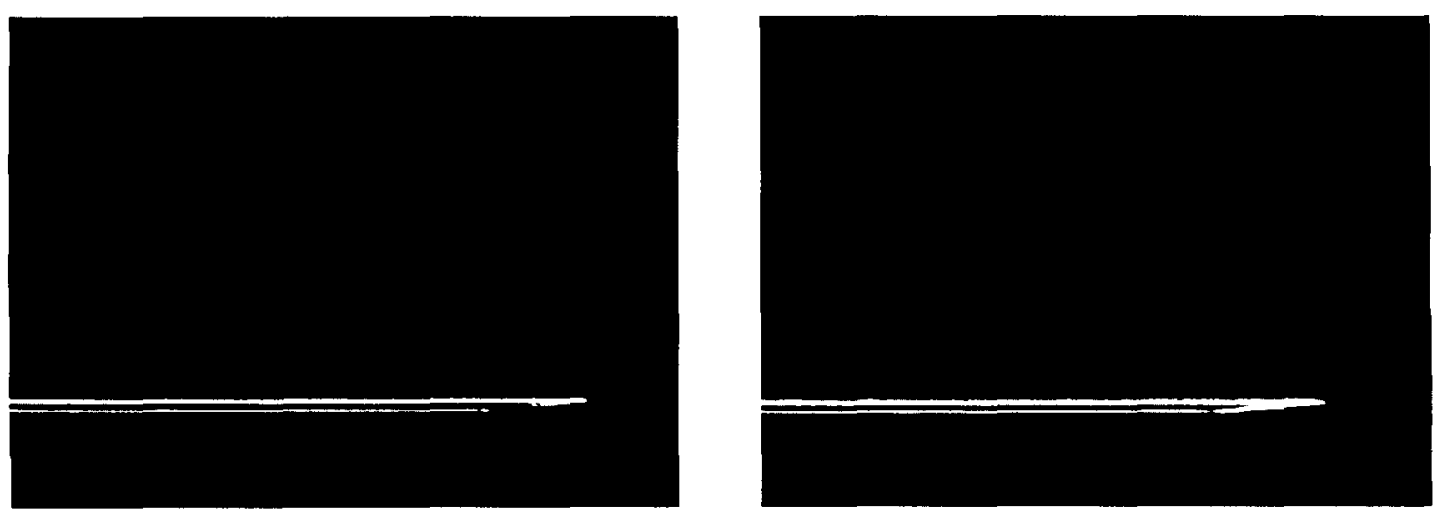

Figure 5.3. $65^{\circ}$ wing, $\alpha=5^{\circ}$; typical left and right camera views (dewarped) 
In the velocity vector images that follow, the field of view has been cropped such that the wing leeward surface is on the axis $x=0$.

\subsection{Resume of velocity vector field plots}

In the following, 3-component vector plots of the velocity field in planar slices are presented; the domain is always a single plane, but the data are three-dimensional. The colorbar legend is with respect to the total velocity magnitude. An instantaneous data set, obtained from one stereo PIV data point, is presented first for each case. A view of the crossflow plane looking upstream is given first, followed by a "top view" looking down onto the wing planform. Then, this is followed by upstream views of an "averaged" and "rms" image, obtained from 100 instantaneous images. Taking the root mean square of such data may not be especially meaningful, but it is some indication of unsteadiness especially local regions of particular unsteadiness, which may happen to be for example in the shear layer emanating from the leading edge, or in the region of LEV-leeward boundary layer interaction.

The plots are arranged in order of increasing angle of attack. With increasing angle of attack, the leading edge vortex becomes larger (when viewed in the streamwise direction), but of course remains in the general area between the wing centerline and [right side] leading edge. Details of vortex core trajectory are described further in Chapter 7. All data are at $z / c=0.3$, for both wings.

\subsection{1 $65^{\circ}$ wing plots}

\subsubsection{1 $65^{\circ}$ Wing. $\alpha=5^{\circ}$}




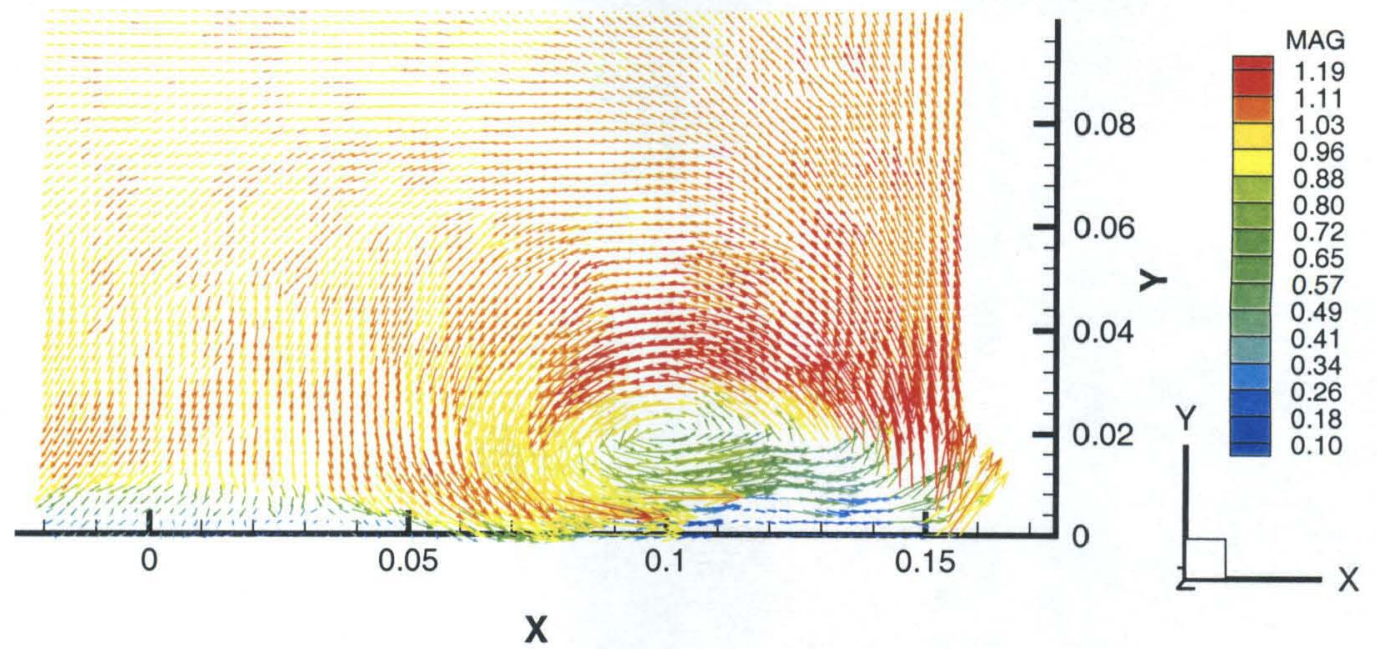

Figure 5.4. $65^{\circ}$ wing, $\alpha=5^{\circ}$, instantaneous velocity field
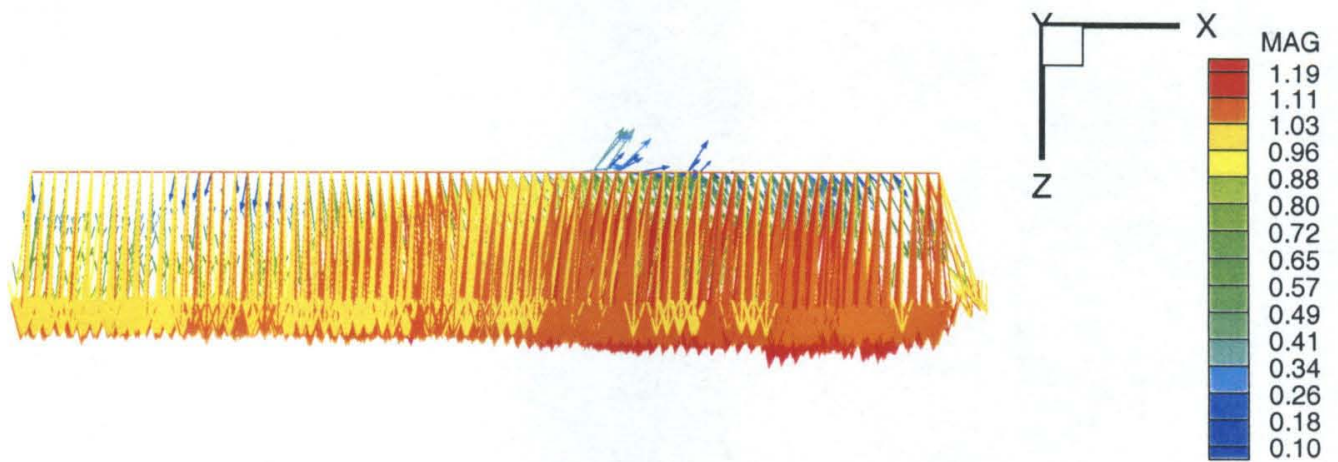

Figure 5.5. $65^{\circ}$ wing, $\alpha=5^{\circ}$, instantaneous velocity field, top view

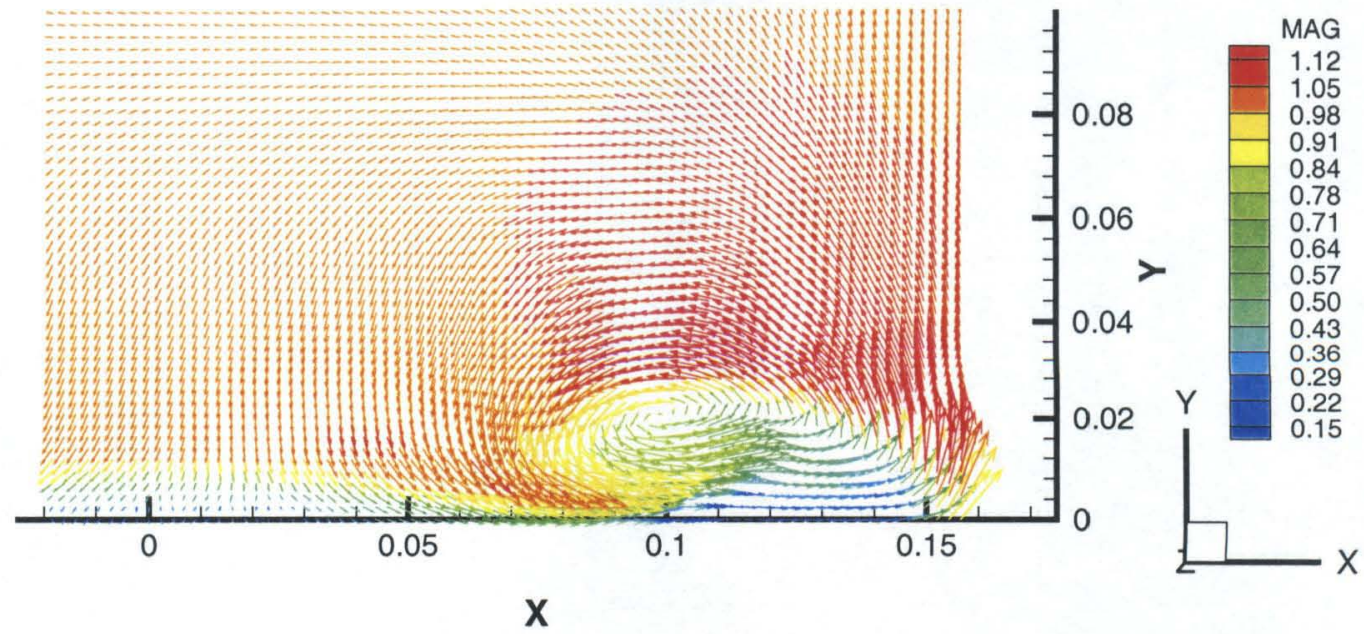

Figure 5.6. $65^{\circ}$ wing, $\alpha=5^{\circ}$, mean velocity field 


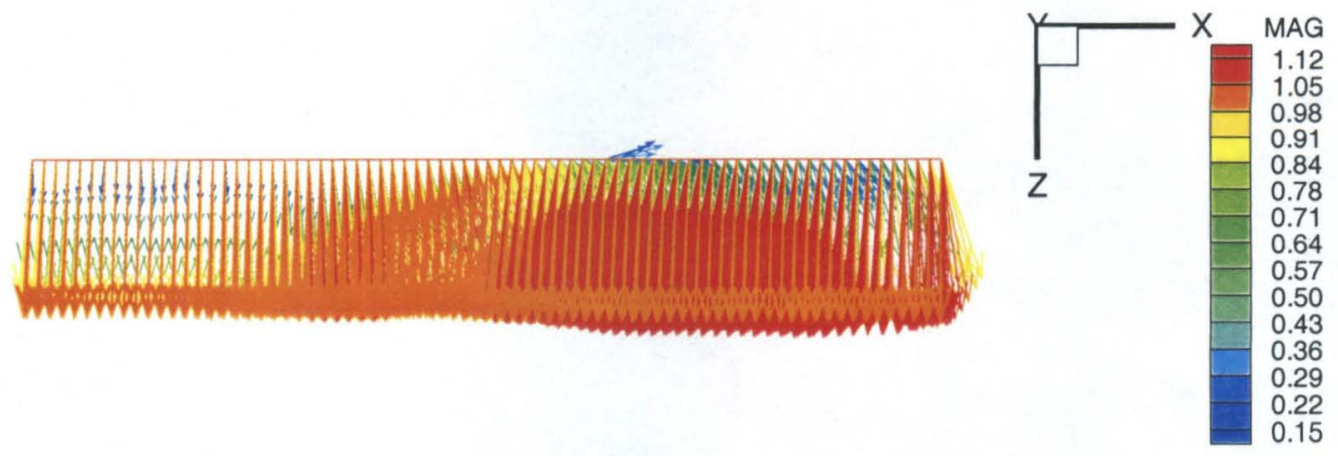

Figure 5.7. $65^{\circ}$ wing, $\alpha=5^{\circ}$, mean velocity field, top view

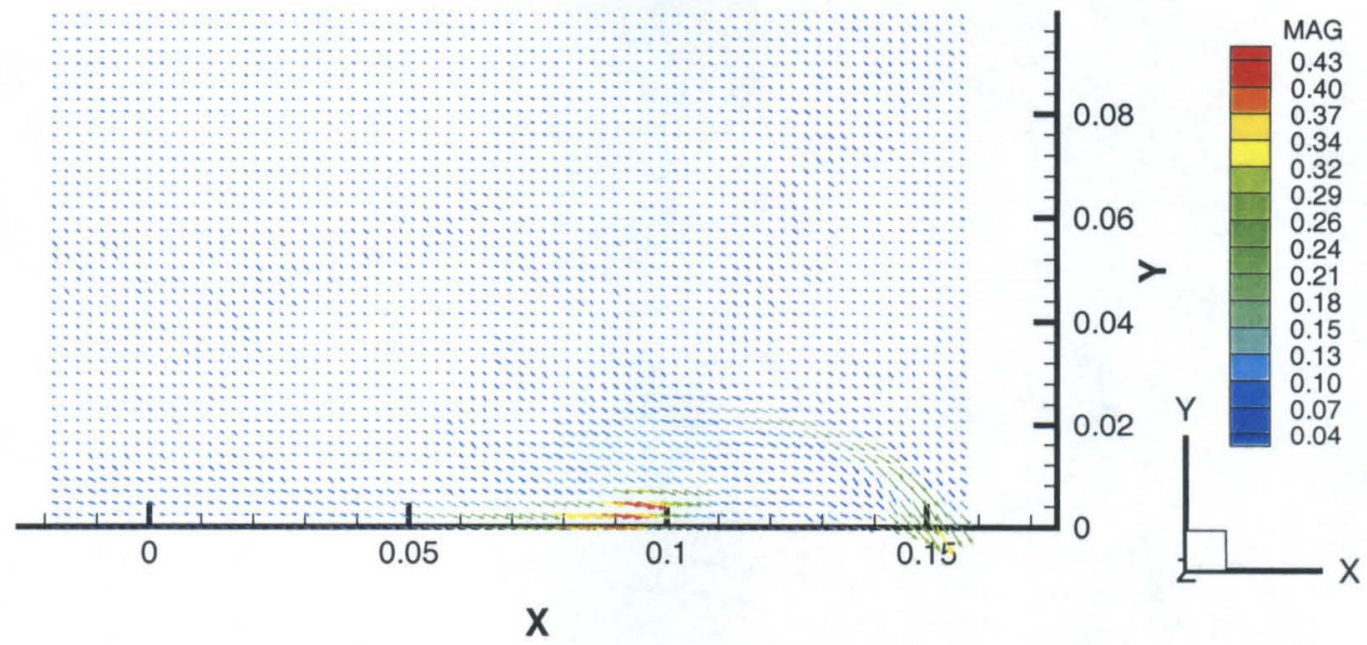

Figure 5.8. $65^{\circ}$ wing, $\alpha=5^{\circ}$, rms velocity field

At this very low angle of attack, leading edge shear layer rollup into a coherent LEV is already visible. The LEV is "flattened" about the wing leeward side. A secondary "vortex" is vaguely discernable. Its sense of rotation is not clear. The mean image differs surprisingly little from the instantaneous image, with the obvious exception of smoothing of "outlier" vectors. This suggests that the flow is quite steady. The rms velocity plot supports this conclusion, showing that the only region of appreciable fluctuations occurs in the portion of the leeward boundary layer near the primary LEV attachment line. 
5.3.1.2 $65^{\circ}$ wing. $\alpha=7.5^{\circ}$

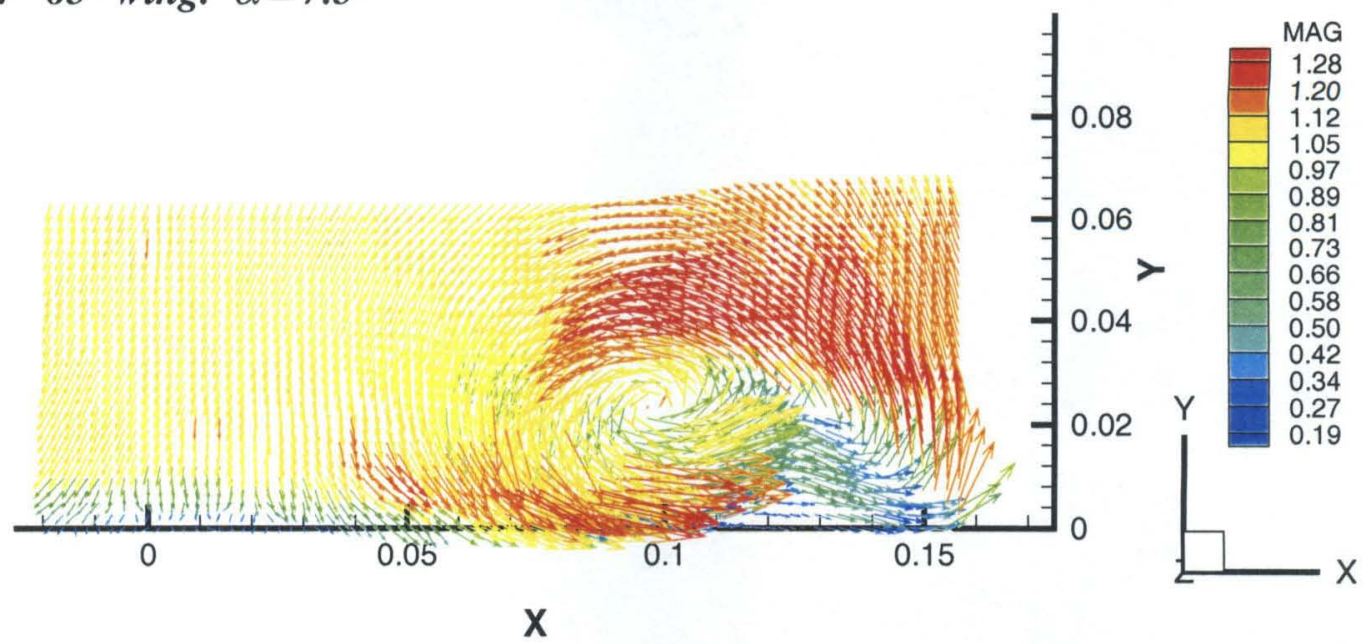

Figure 5.9. $65^{\circ}$ wing, $\alpha=7.5^{\circ}$, instantaneous velocity field
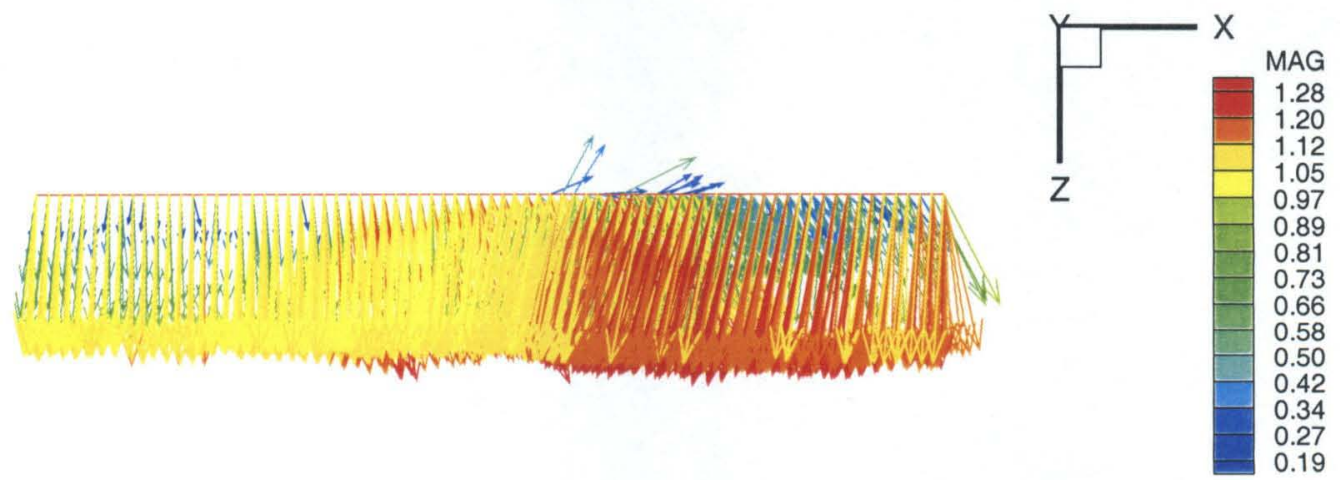

Figure 5.10. $65^{\circ}$ wing, $\alpha=7.5^{\circ}$, instantaneous velocity field, top view

Here the core axial velocity is still not much higher than free stream.

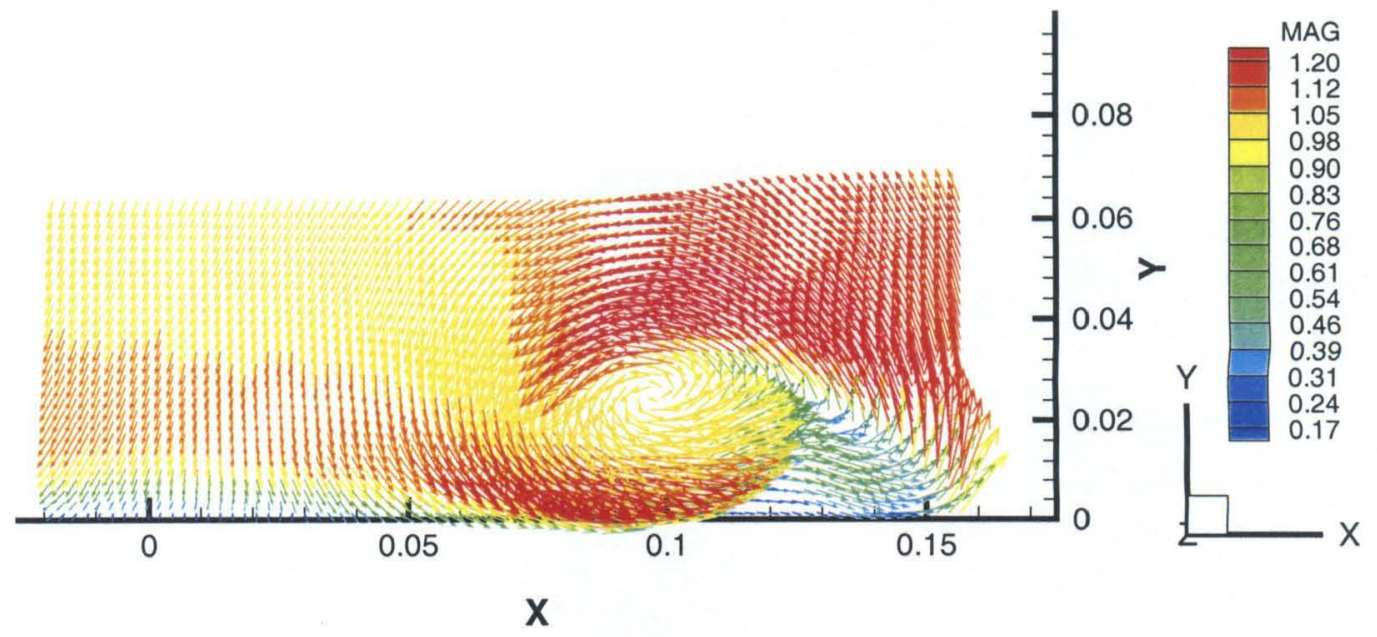

Figure 5.11. $65^{\circ}$ wing, $\alpha=7.5^{\circ}$, mean velocity field 


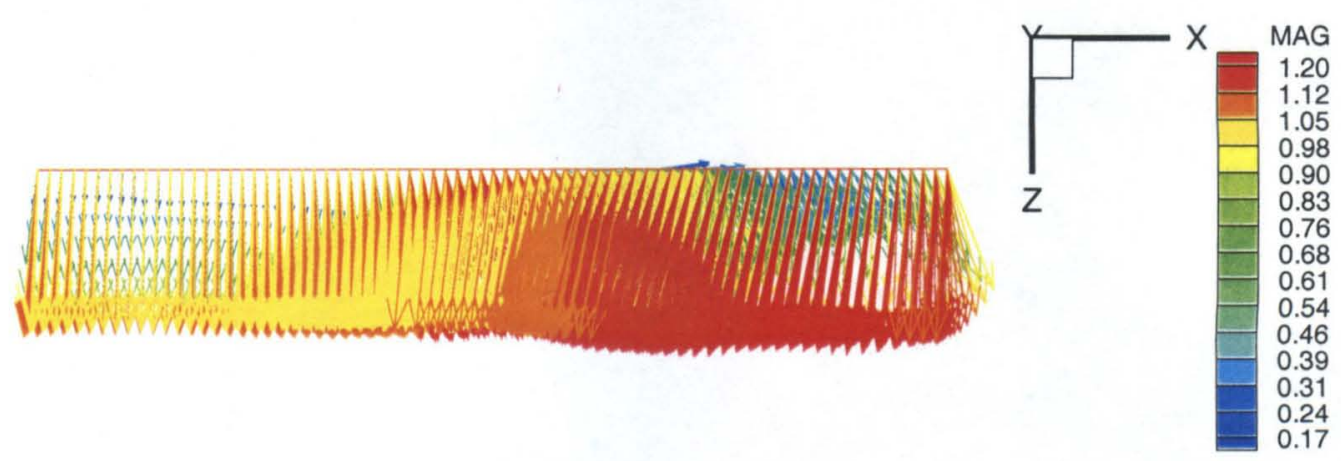

Figure 5.12. $65^{\circ}$ wing, $\alpha=7.5^{\circ}$, mean velocity field, top view

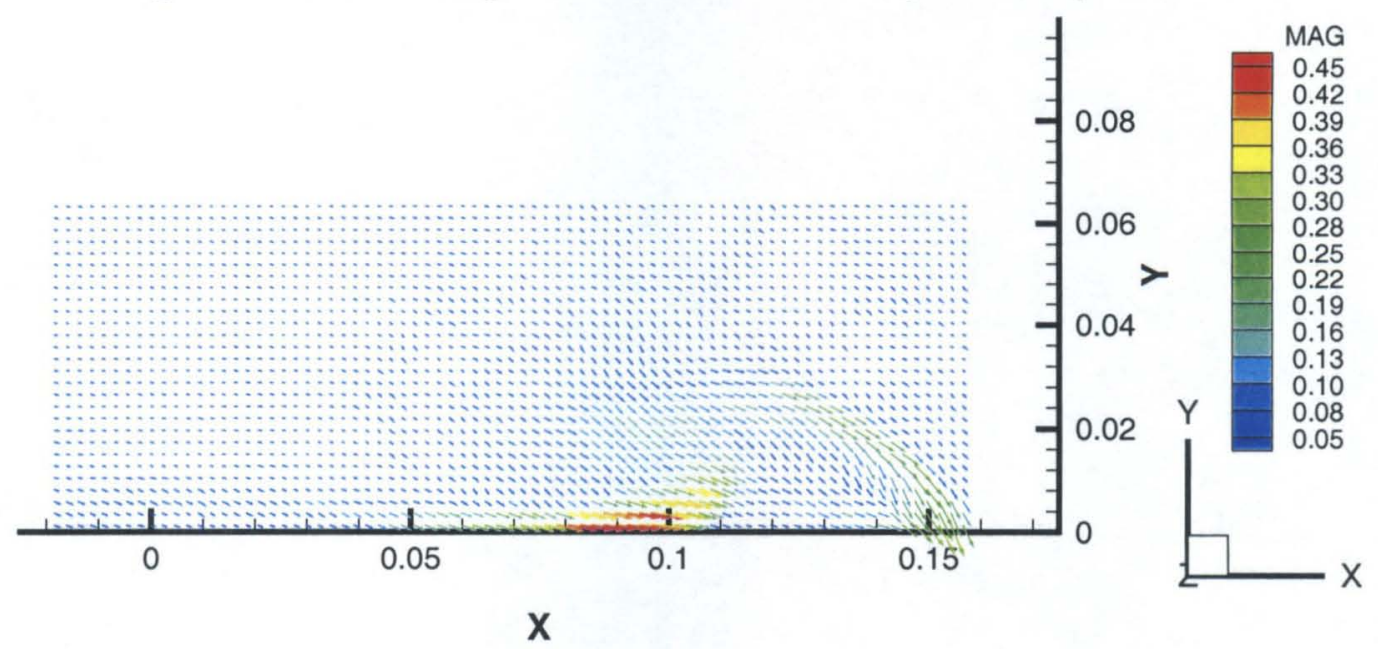

Figure 5.13. $65^{\circ}$ wing, $\alpha=7.5^{\circ}$, rms velocity field

This case is qualitatively similar to the $5^{\circ}$ case. The LEV is now of greater vertical extent.

5.3.1.3 $65^{\circ}$ wing. $\alpha=10^{\circ}$ 


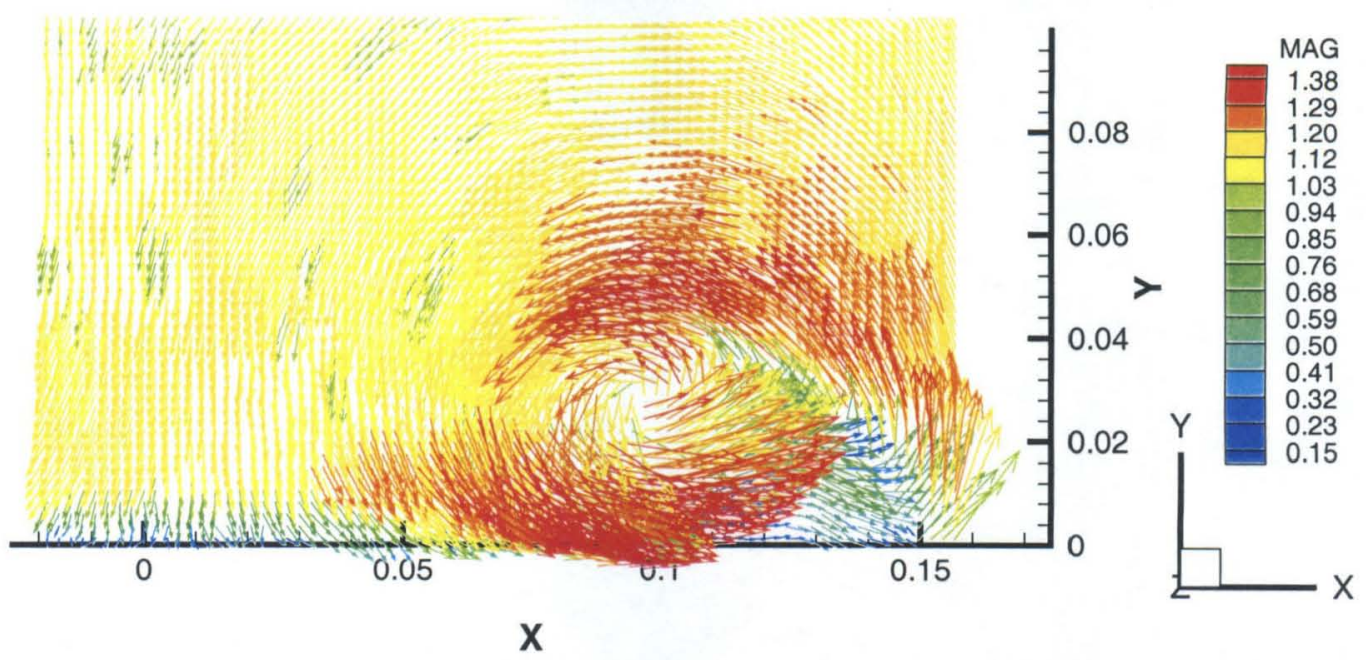

Figure 5.14. $65^{\circ}$ wing, $\alpha=10^{\circ}$, instantaneous velocity field

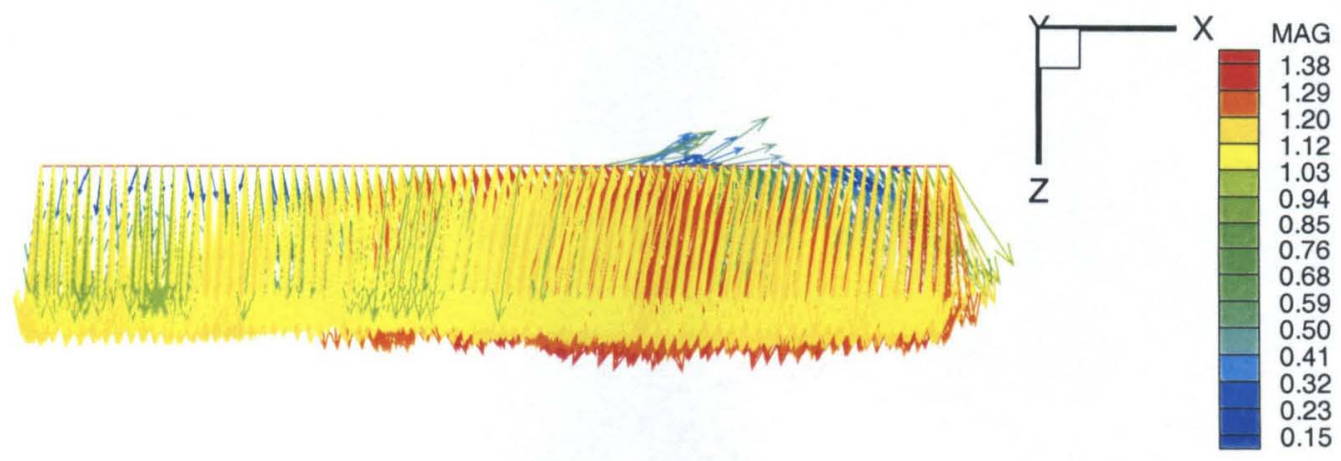

Figure 5.15. $65^{\circ}$ wing, $\alpha=10^{\circ}$, instantaneous velocity field, top view

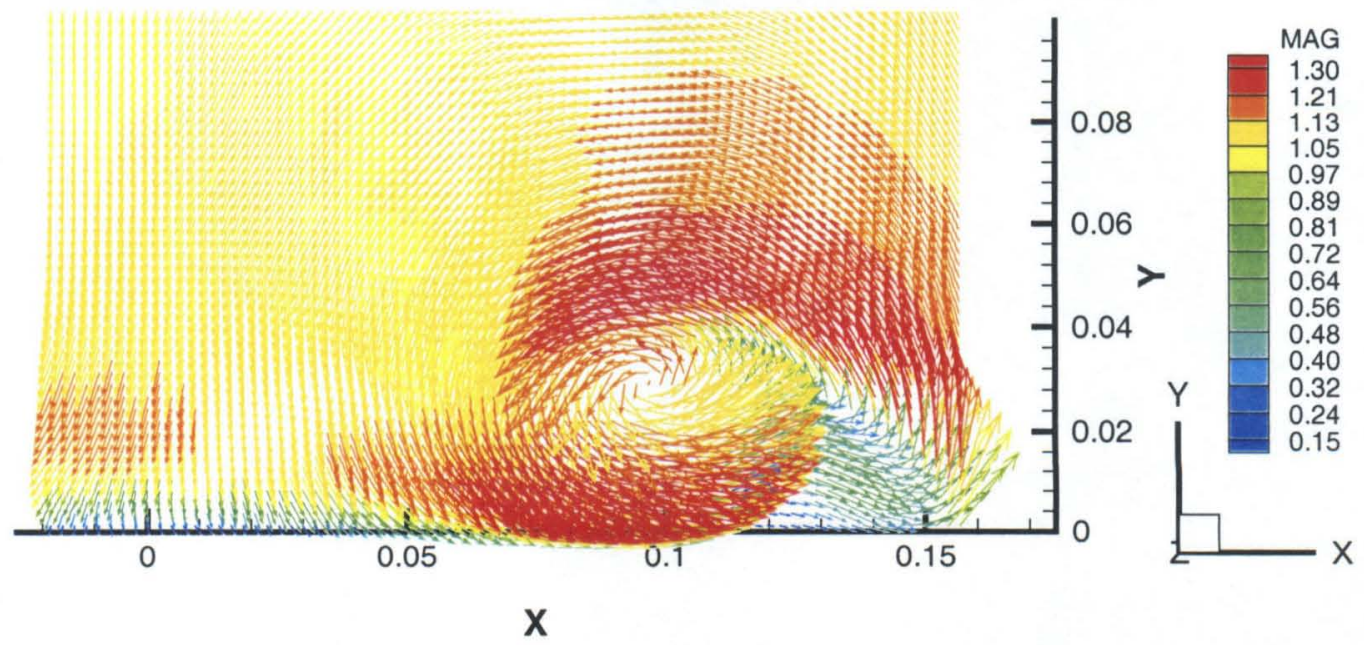

Figure 5.16. $65^{\circ}$ wing, $\alpha=10^{\circ}$, mean velocity field 


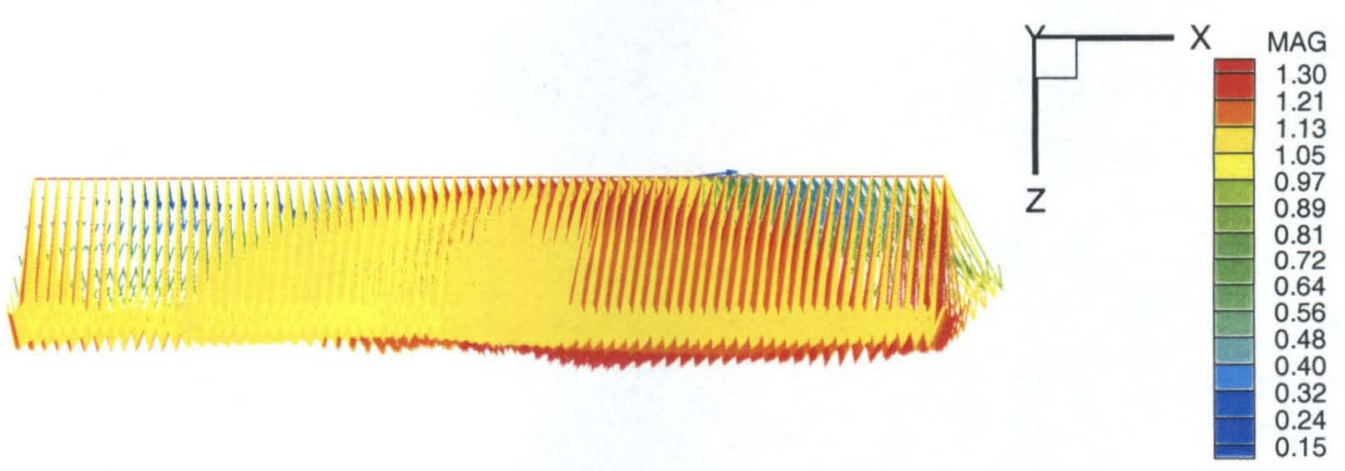

Figure 5.17. $65^{\circ}$ wing, $\alpha=10^{\circ}$, mean velocity field, top view

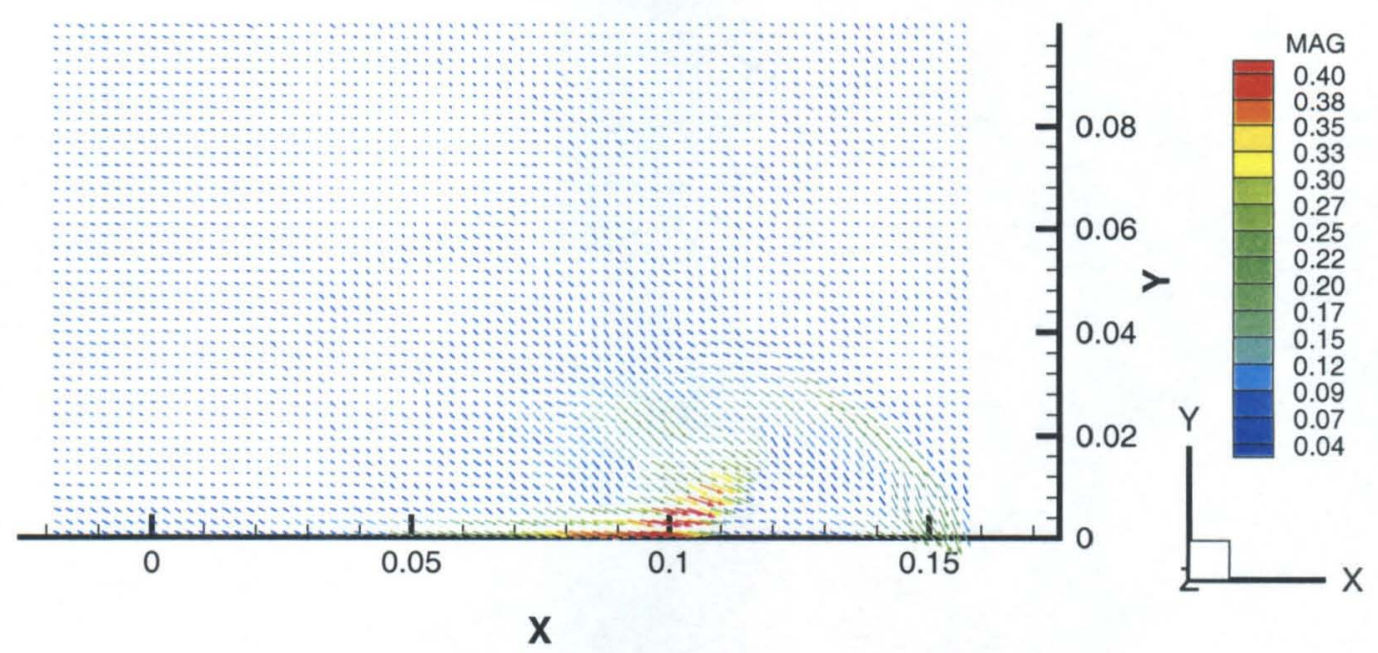

Figure 5.18. $65^{\circ}$ wing, $\alpha=10^{\circ}$, rms velocity field

Again, this case is similar to the above. There is still some evidence of a secondary vortex. The primary LEV continues to increase in vertical extent, and now is starting to approach the classical picture of a delta wing at high angle of attack. However, the streamwise velocity component distribution in the LEV is still not sufficiently pronounced to be called a "jet-like" profile.

5.3.1.4 $65^{\circ}$ wing. $\alpha=12.5^{\circ}$ 


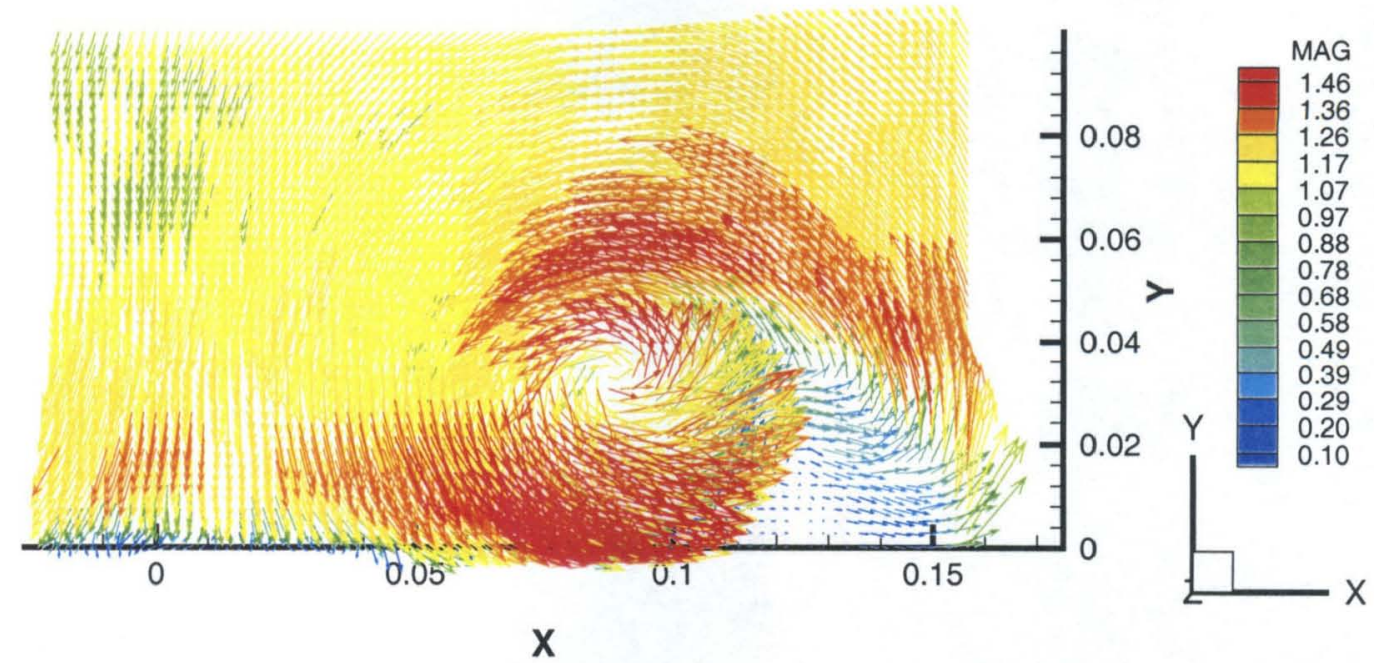

Figure 5.19. $65^{\circ}$ wing, $\alpha=12.5^{\circ}$, instantaneous velocity field
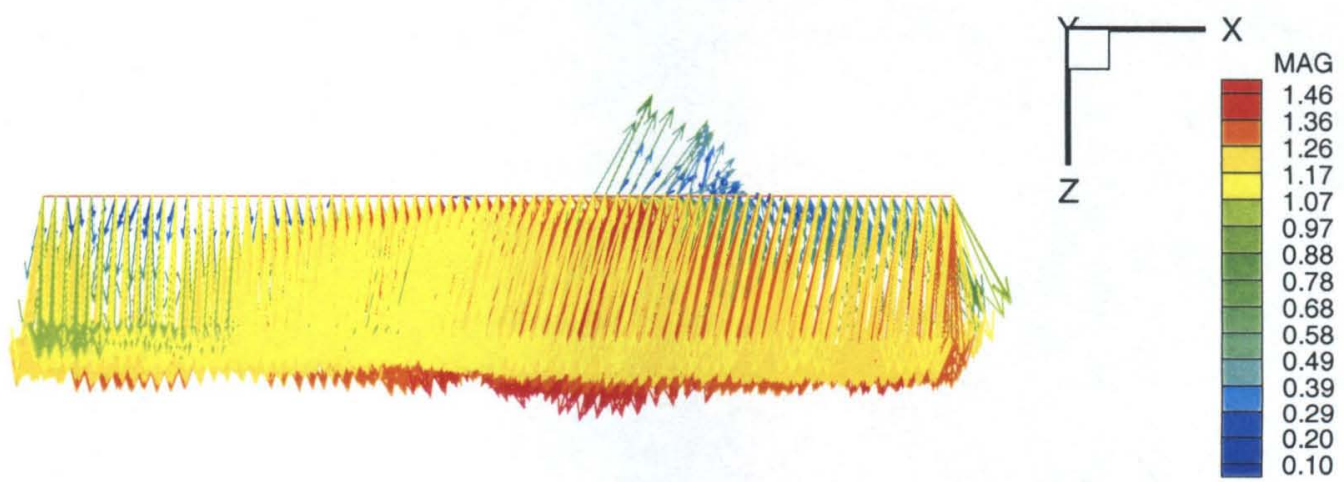

Figure 5.20. $65^{\circ}$ wing, $\alpha=12.5^{\circ}$, instantaneous velocity field, top view

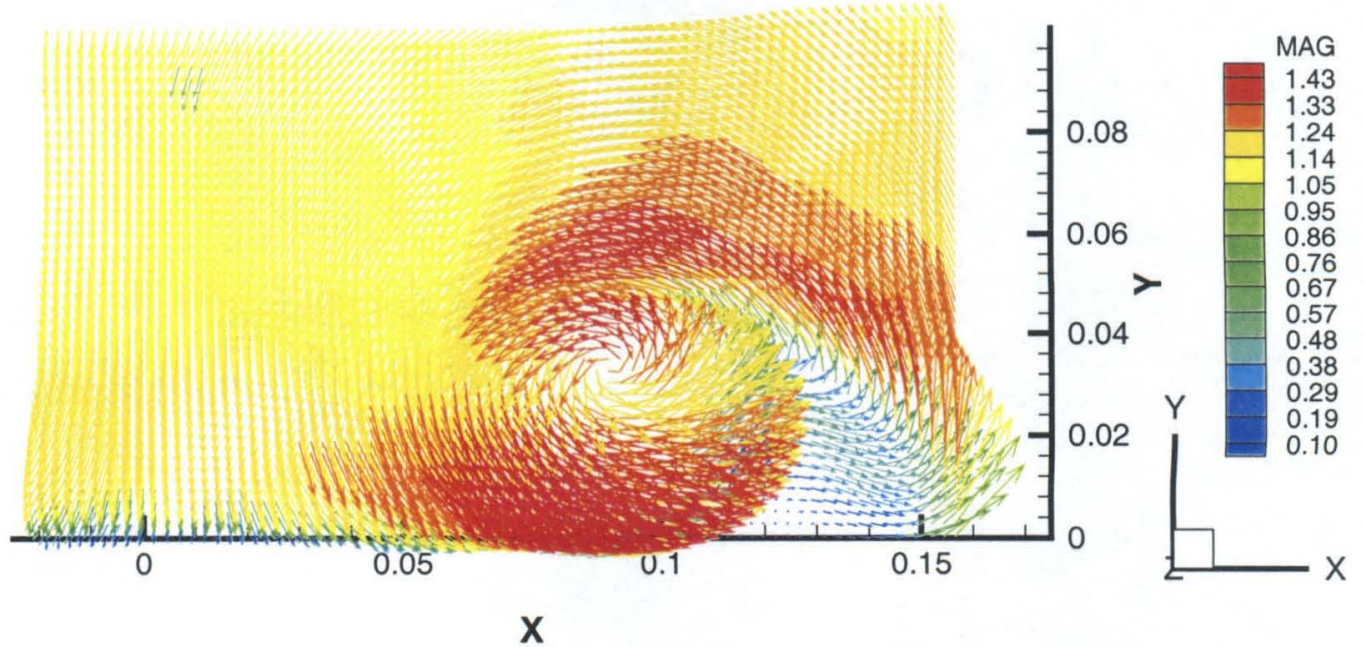

Figure 5.21. $65^{\circ}$ wing, $\alpha=12.5^{\circ}$, mean velocity field 


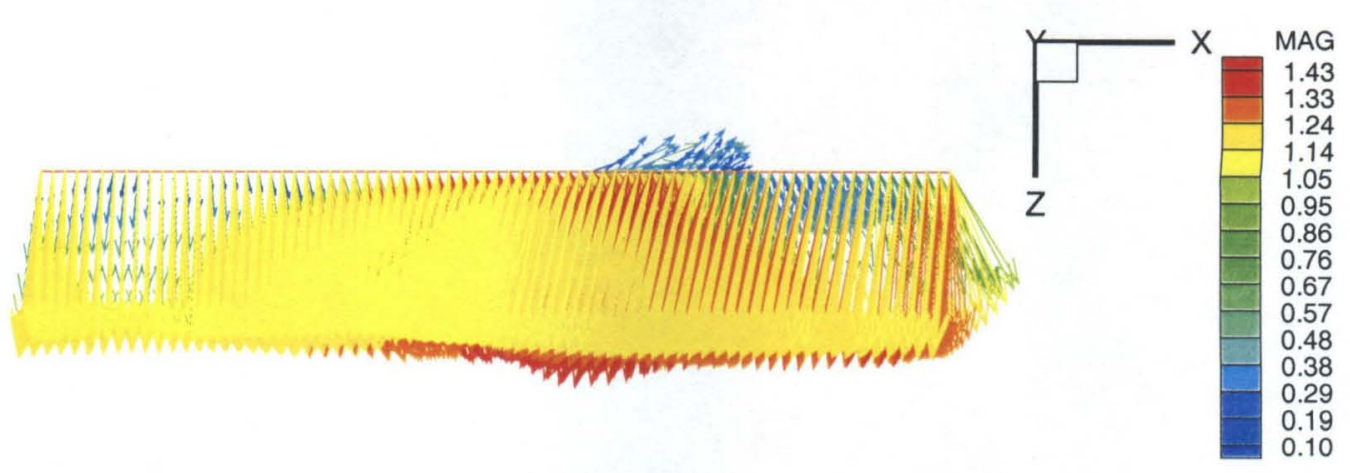

Figure 5.22. $65^{\circ}$ wing, $\alpha=12.5^{\circ}$, mean velocity field, top view

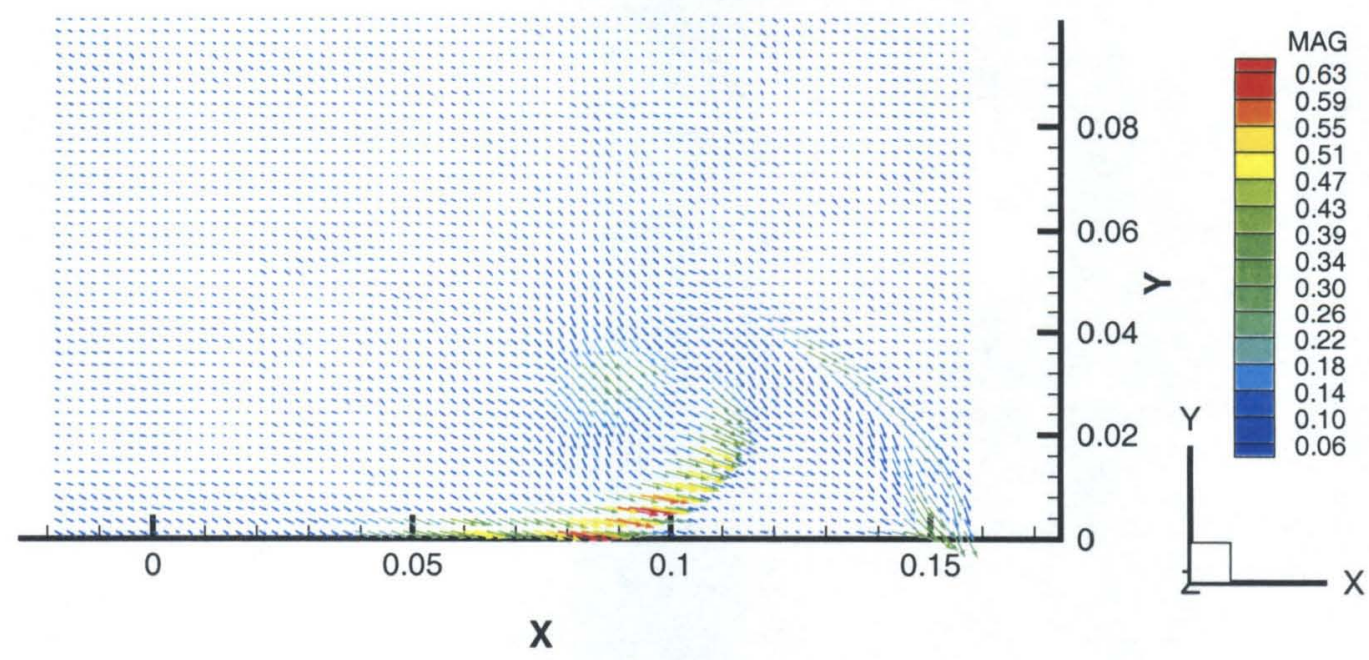

Figure 5.23. $65^{\circ}$ wing, $\alpha=12.5^{\circ}$, rms velocity field

At the $12.5^{\circ}$ angle of attack, somewhat stronger changes in the flowfield are apparent, than those in the previous cases. The primary LEV core axial velocity is discernibly higher than free stream velocity. Outboard of the primary LEV, motion near the leeward surface, identifiable with a secondary vortex, is notable attenuated. This region is now dominated by what appears to be stagnation. Just outboard of the primary LEV attachment line, there appears to be a small region of flow reversal. The rms plot shows that the region of strong unsteadiness has extended away from the wing surface, and now follows the curvature of the primary LEV in going away from its contact point with the wing leeward surface. 
5.3.1.5 $65^{\circ}$ wing. $\alpha=15^{\circ}$

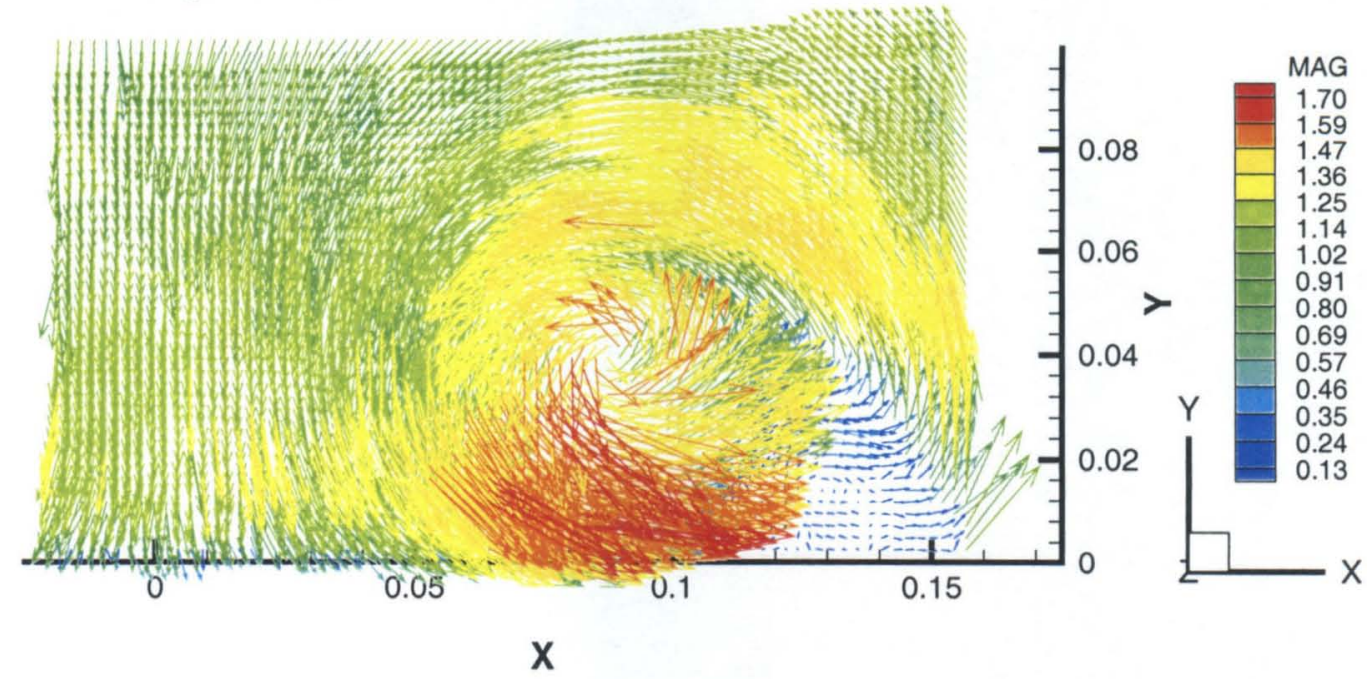

Figure 5.24. $65^{\circ}$ wing, $\alpha=15^{\circ}$, instantaneous velocity field

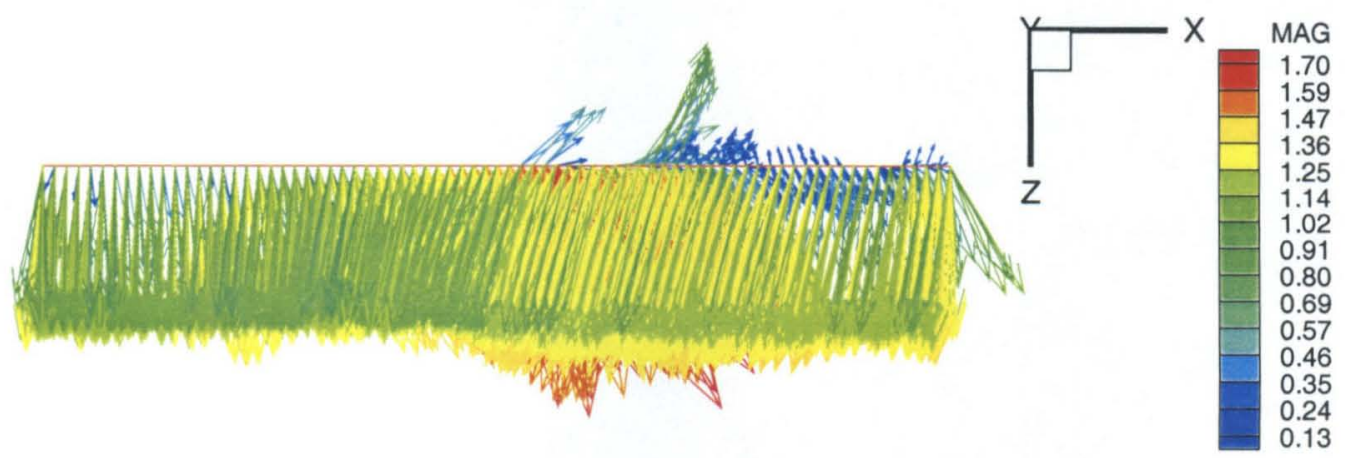

Figure 5.25. $65^{\circ}$ wing, $\alpha=15^{\circ}$, instantaneous velocity field, top view

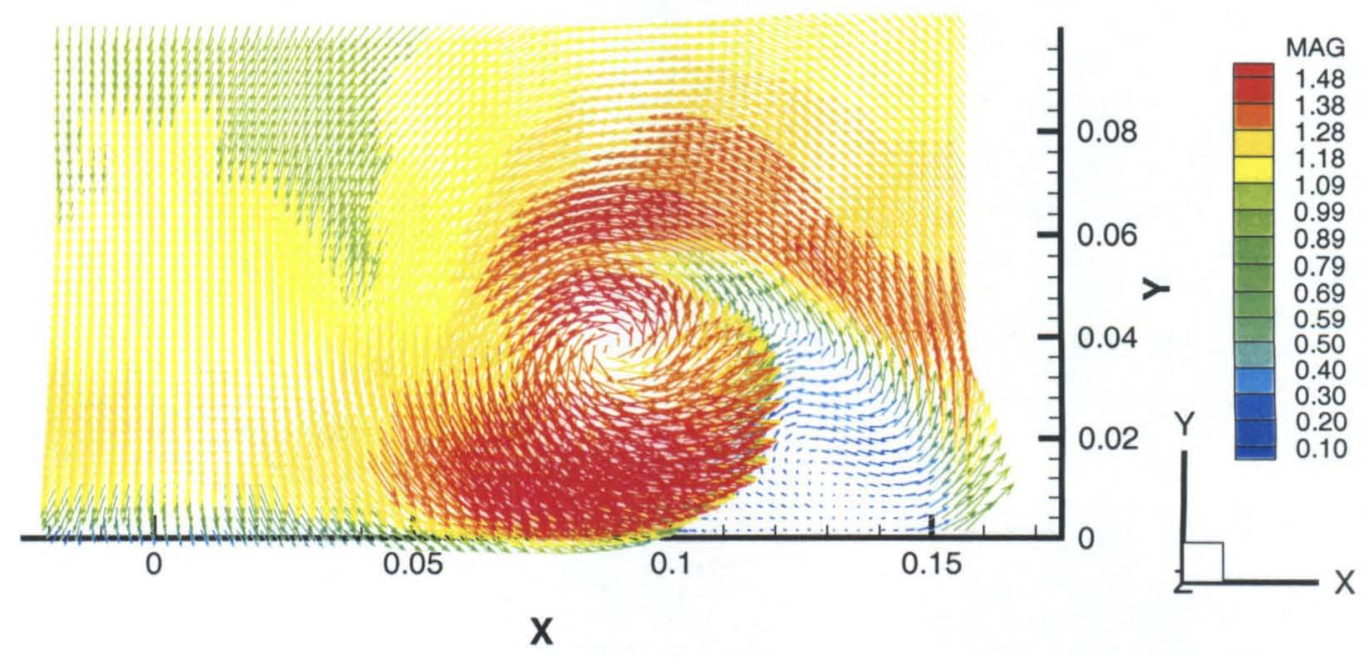

Figure 5.26. $65^{\circ}$ wing, $\alpha=15^{\circ}$, mean velocity field 


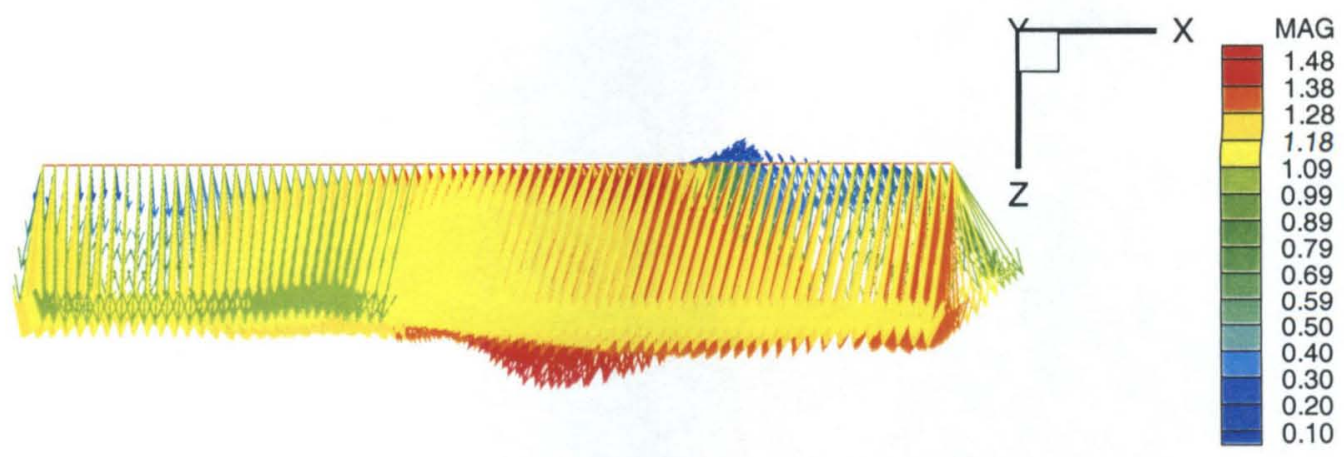

Figure 5.27. $65^{\circ}$ wing, $\alpha=15^{\circ}$, mean velocity field, top view

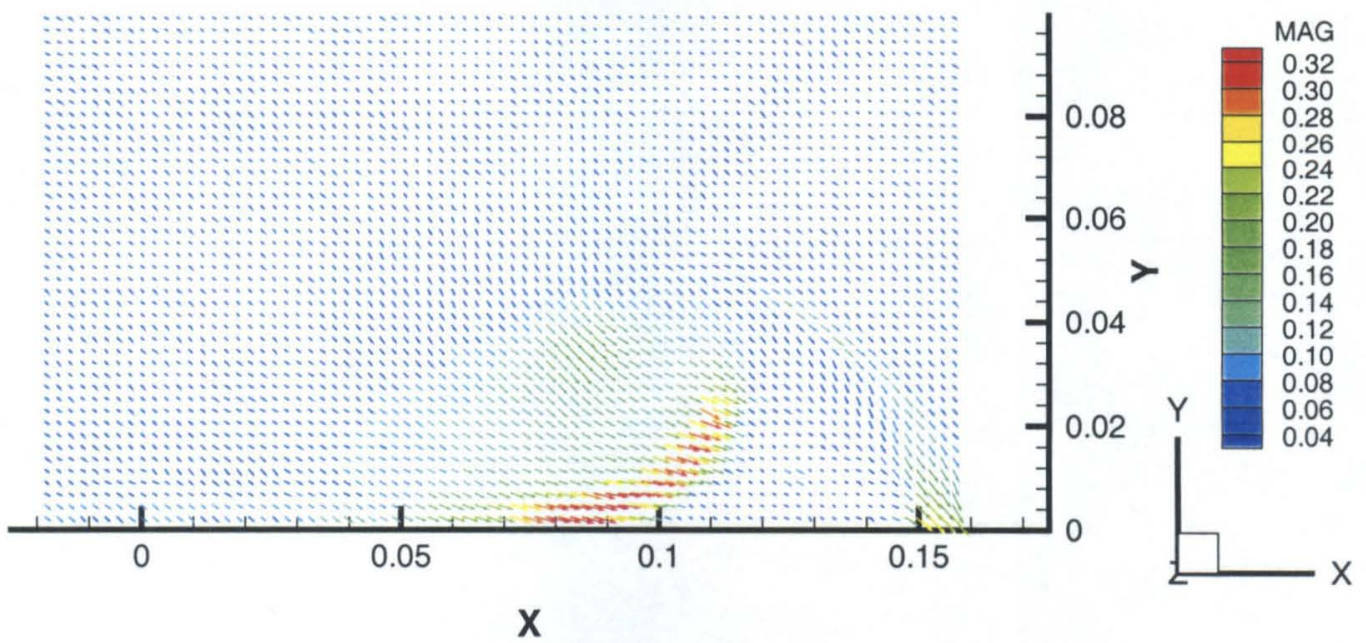

Figure 5.28. $65^{\circ}$ wing, $\alpha=15^{\circ}$, rms velocity field

Here the core axial velocity is yet higher. The outboard stagnation region has grown in extent, completely dominating flow outboard of the primary LEV. From the rms plot, it is seen that appreciable unsteadiness is also increasing, now starting to cover a region internal to the $\mathrm{LEV}$ itself.

5.3.1.6 $65^{\circ}$ wing. $\alpha=20^{\circ}$ 


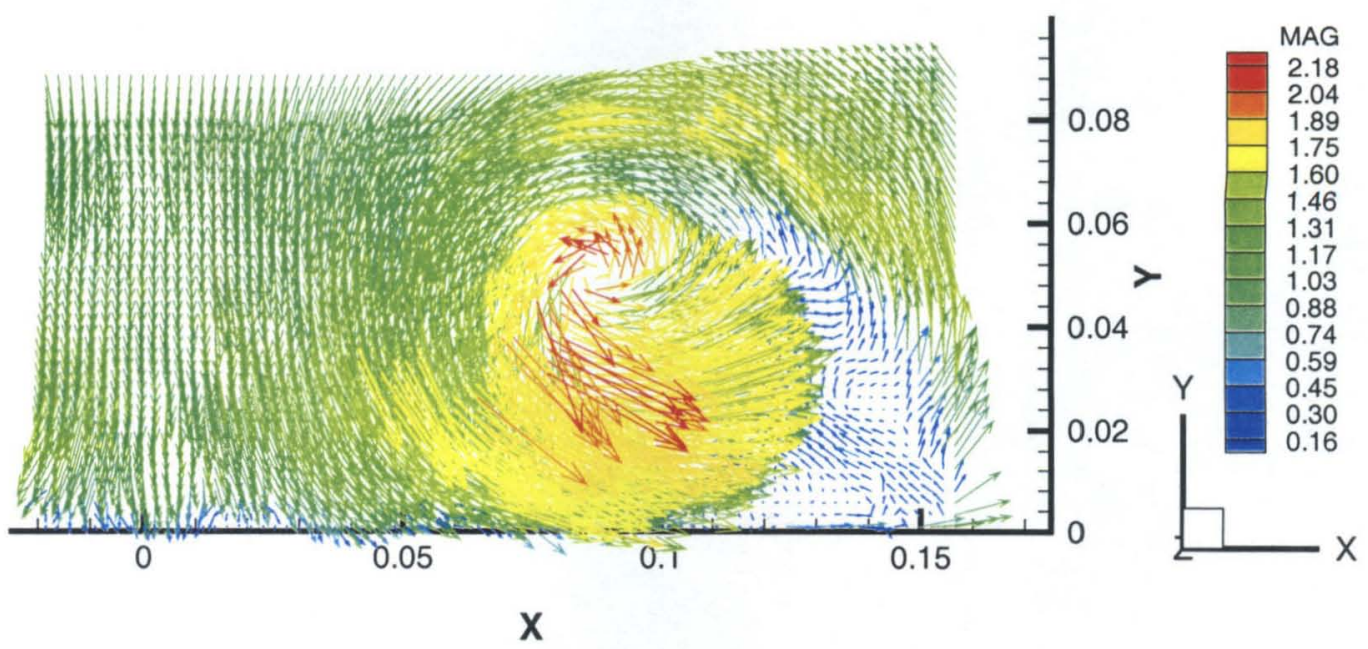

Figure 5.29. $65^{\circ}$ wing, $\alpha=20^{\circ}$, instantaneous velocity field

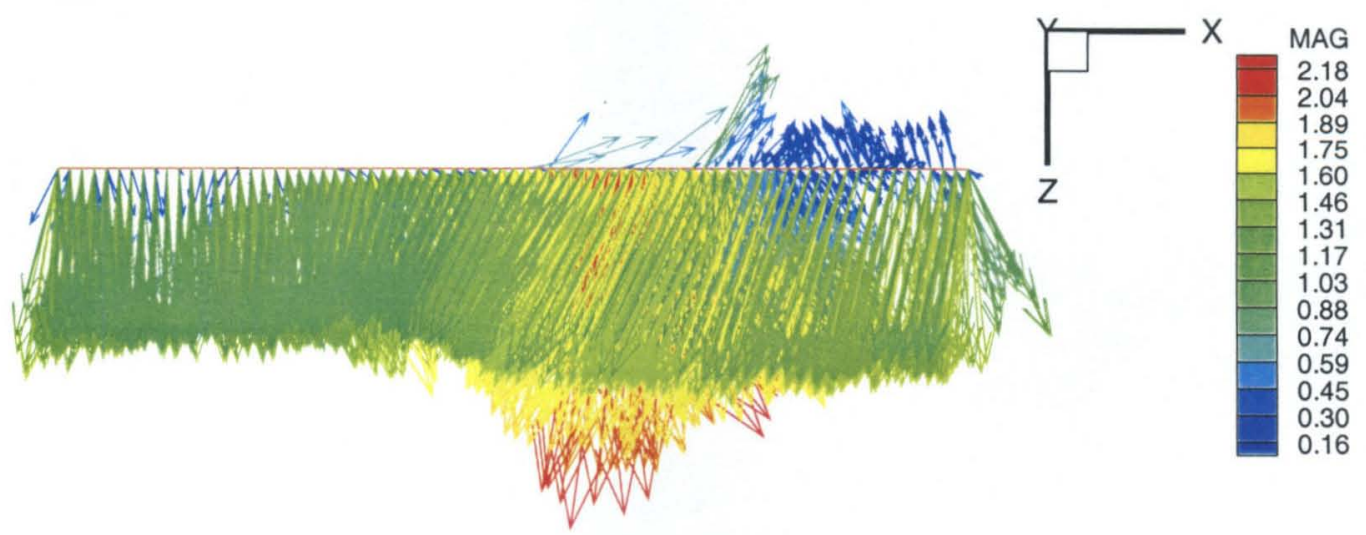

Figure 5.30. $65^{\circ}$ wing, $\alpha=20^{\circ}$, instantaneous velocity field, top view

Here we finally see a more obvious jet-like axial velocity profile in the LEV.

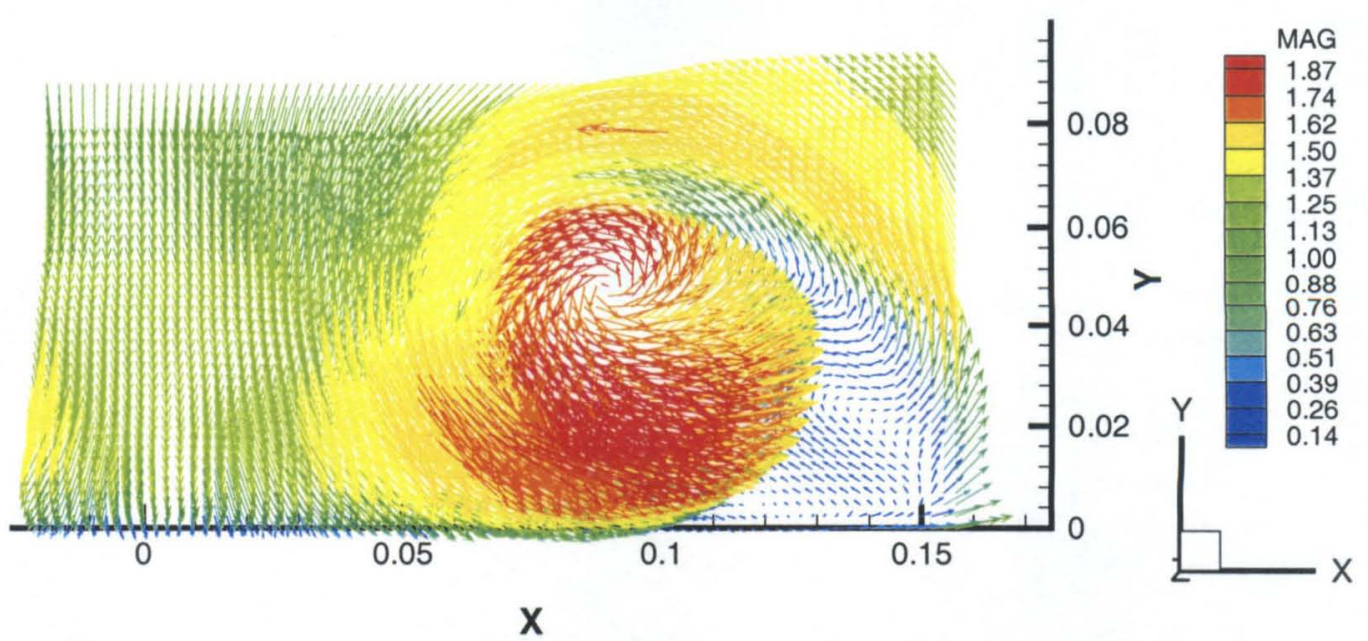

Figure 5.31. $65^{\circ}$ wing, $\alpha=20^{\circ}$, mean velocity field 


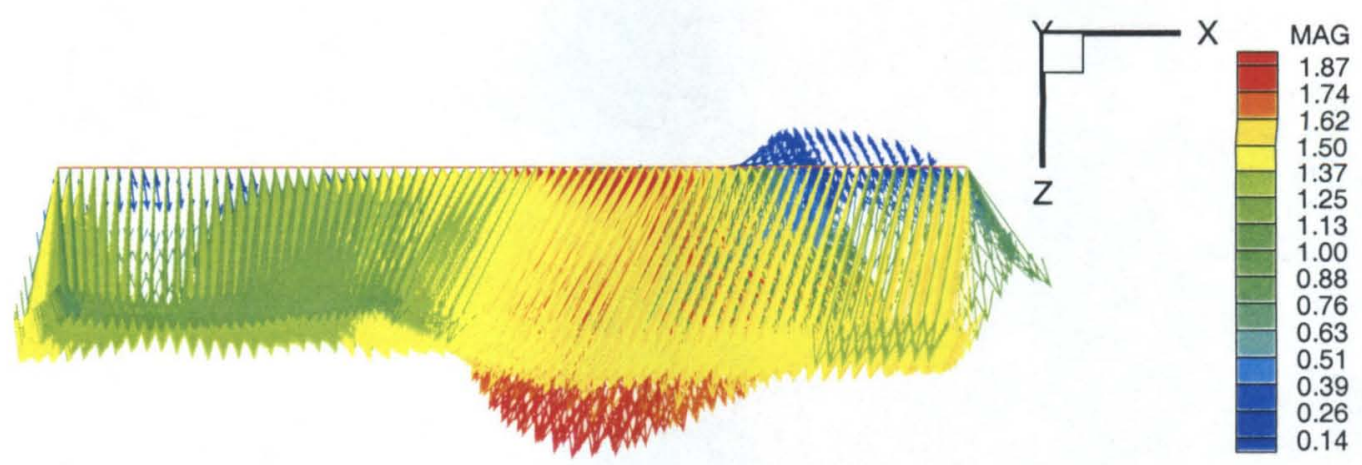

Figure 5.32. $65^{\circ}$ wing, $\alpha=20^{\circ}$, mean velocity field, top view

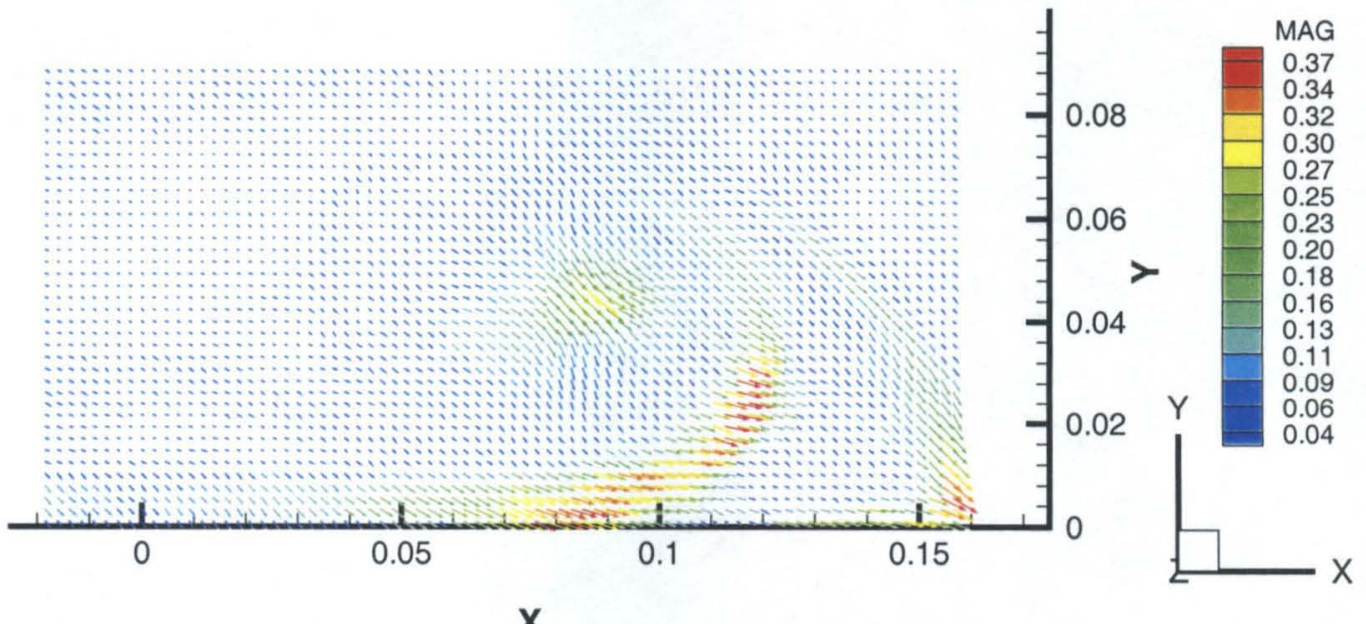

Figure 5.33. $65^{\circ}$ wing, $\alpha=20^{\circ}$, rms velocity field

The core axial velocity distribution for this case is now about double that of the free stream - typical of the situation for a slender wing at high angle of attack, as reported in the literature. Note that the strong peak in the instantaneous case is slightly attenuated when averaged, but is qualitatively unchanged. The apparent bias of the core velocity "hump" toward the left is a consequence of viewing the velocity vectors (which of course have an azimuthal component) from the top. The outboard stagnation region is now seen to actually be a region of flow reversal. Unsteadiness has slightly increased, now encompassing a small region in the immediate vicinity of the leading edge. The main 
region of unsteadiness is the "juncture" between the primary LEV and the outboard stagnation region.

\subsection{2 $50^{\circ}$ wing plots}

This section repeats the format of the above. Averaging is again over 100 images, except for the $\alpha=12.5^{\circ}$ case, noted below.

5.3.2.1 50 ${ }^{\circ}$ wing. $\alpha=5^{\circ}$

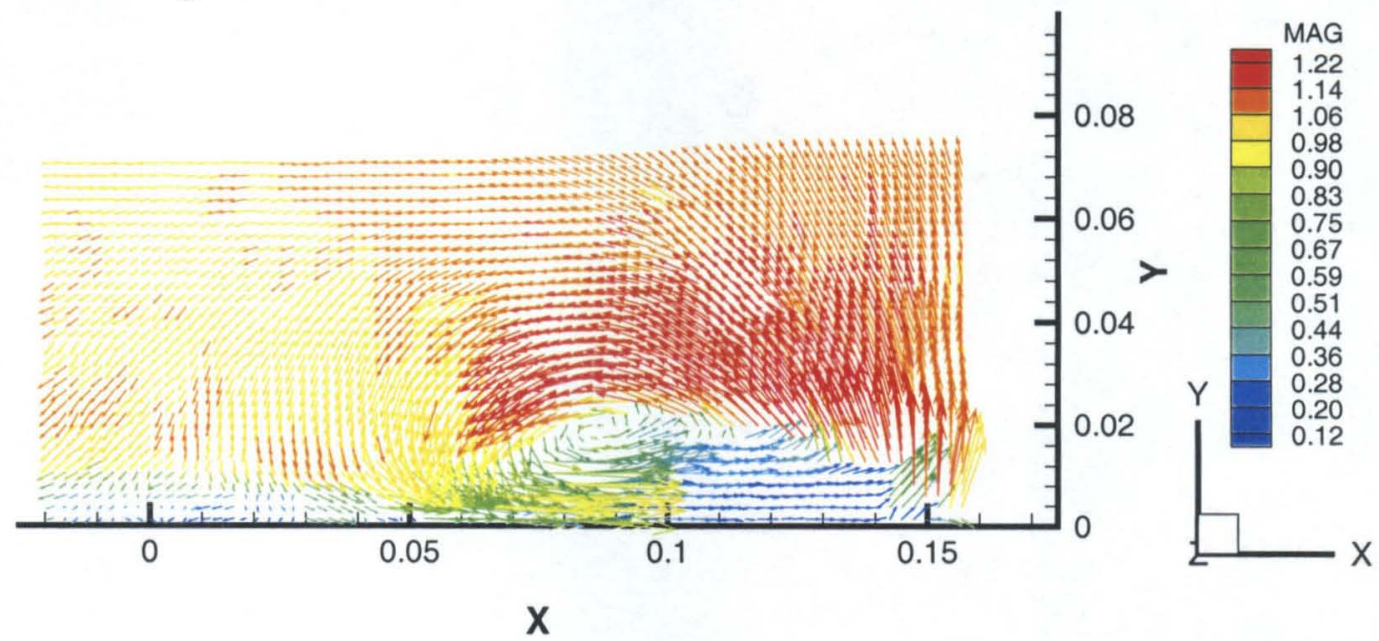

Figure 5.34. $50^{\circ}$ wing, $\alpha=5^{\circ}$, instantaneous velocity field
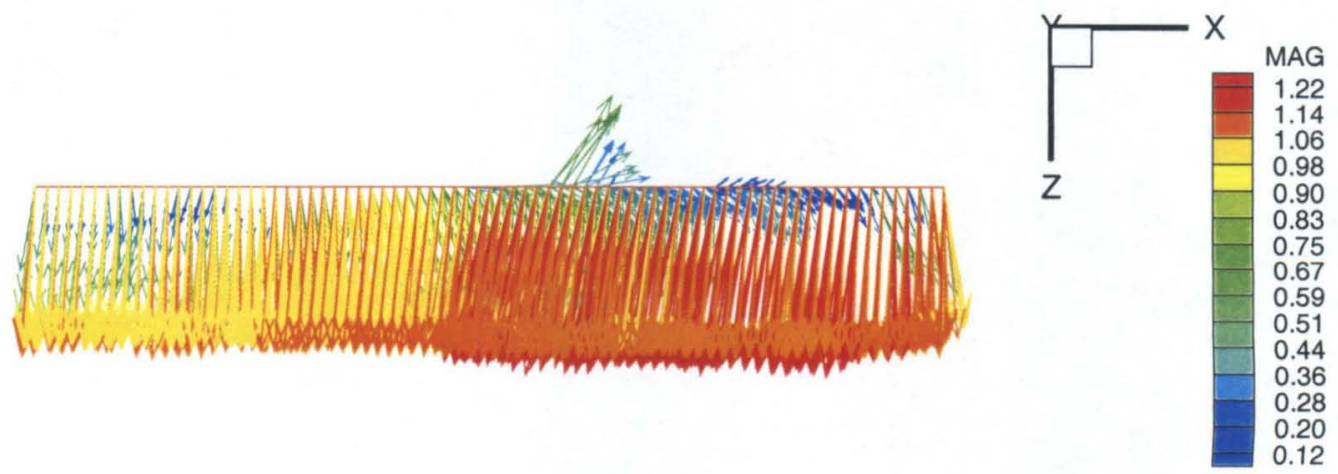

Figure 5.35. $50^{\circ}$ wing, $\alpha=5^{\circ}$, instantaneous velocity field, top view 


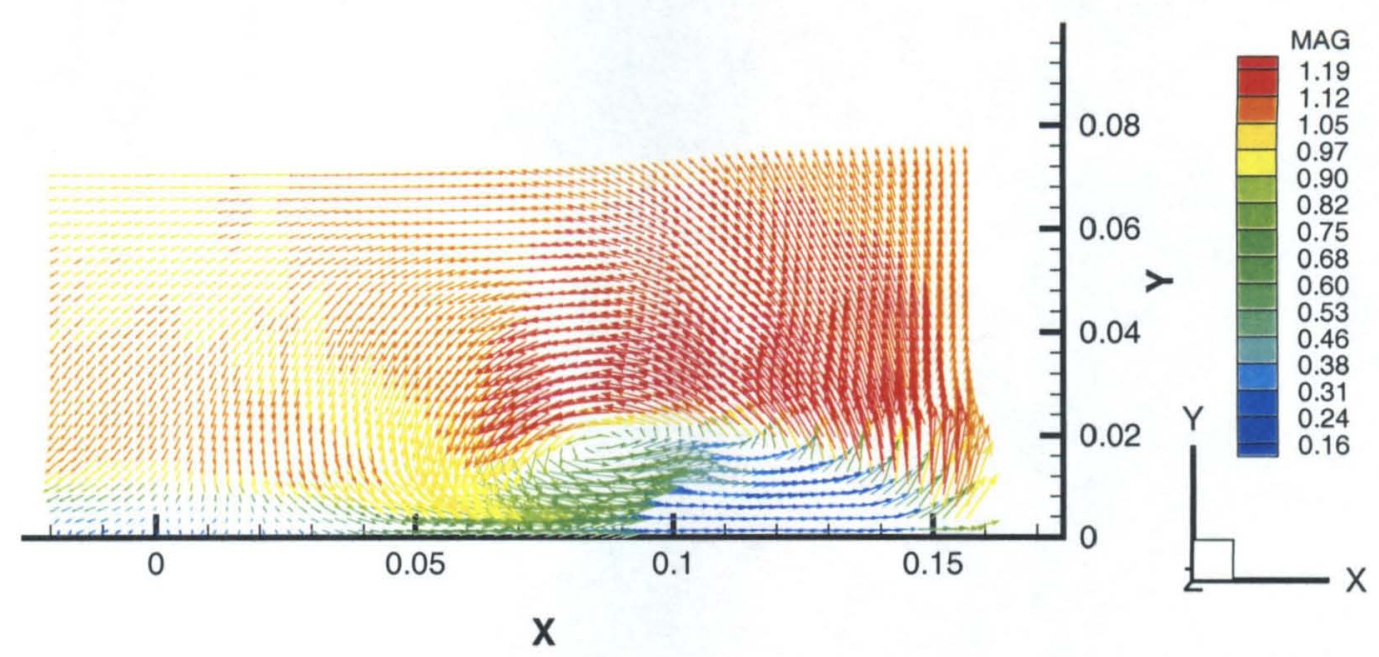

Figure 5.36. $50^{\circ}$ wing, $\alpha=5^{\circ}$, mean velocity field
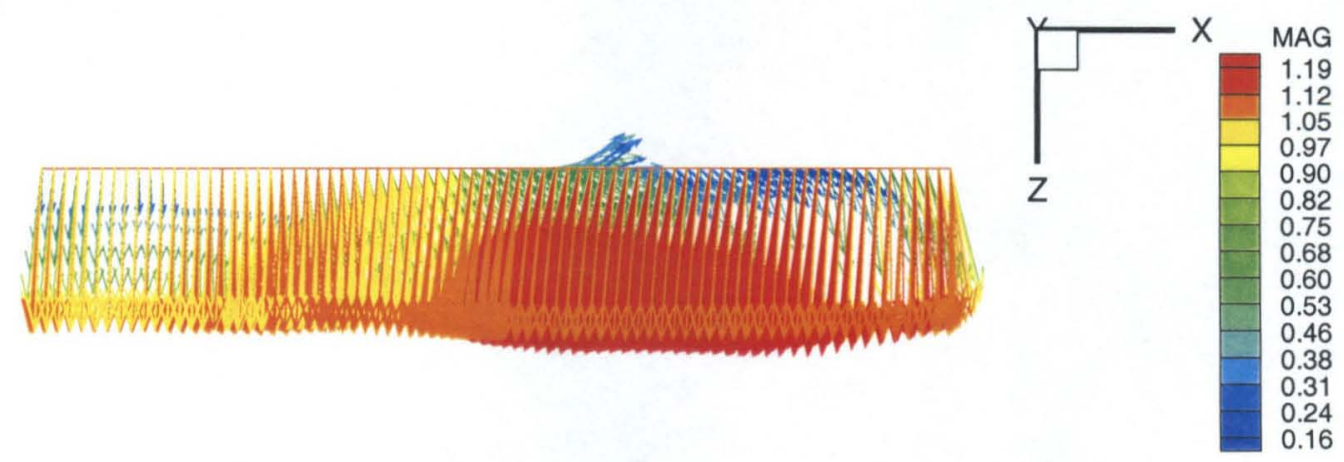

Figure 5.37. $50^{\circ}$ wing, $\alpha=5^{\circ}$, mean velocity field, top view

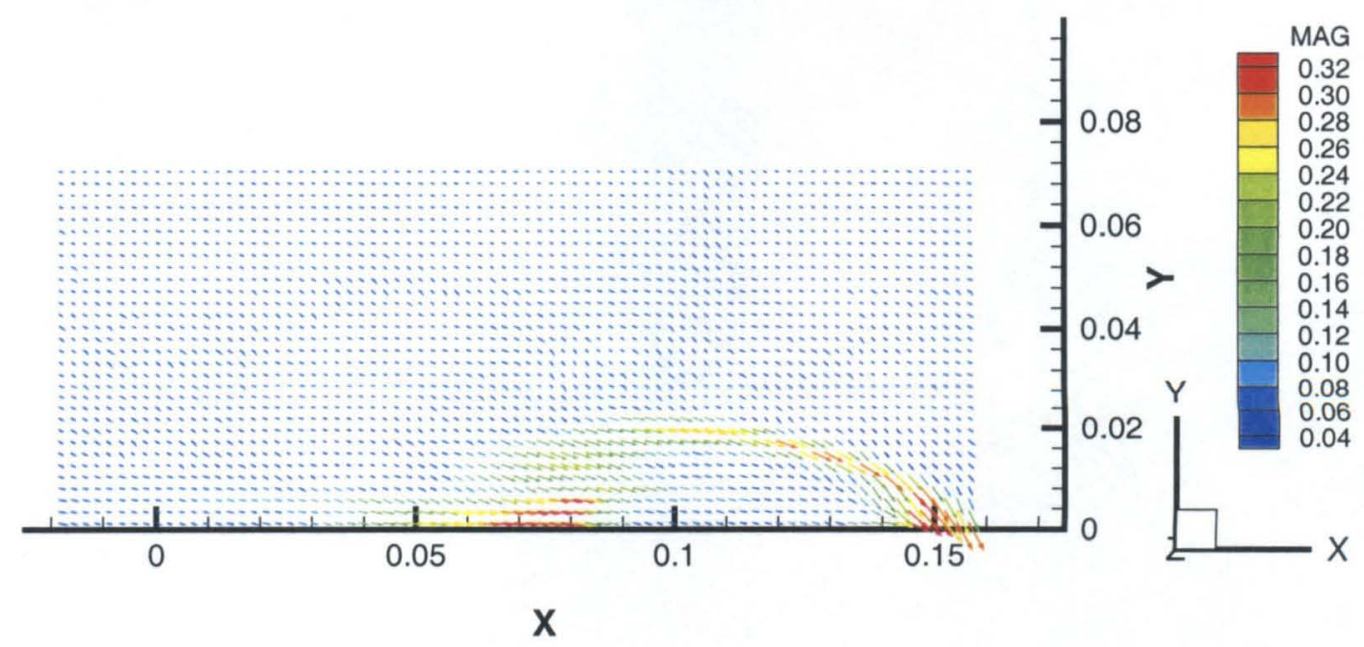

Figure 5.38. 50 $^{\circ}$ wing, $\alpha=5^{\circ}$, rms velocity field 
Qualitatively, this flowfield appears to be similar to that of the $65^{\circ}$ wing, at least in the mean. The primary LEV is definitely visible, even at this low angle of attack. However, it is "flatter" than that of the $65^{\circ}$ wing. Outboard of the primary LEV, secondary flow is weak. From the rms plot, it is seen that appreciable unsteadiness is observed not only in the leeward boundary layer/primary LEV attachment region, but throughout the rolling-up shear layer, and especially at the leading edge. In that regard, the flow is similar to that of the $65^{\circ}$ wing at $20^{\circ}$ angle of attack.

\subsubsection{2 $50^{\circ}$ wing. $\alpha=7.5^{\circ}$}

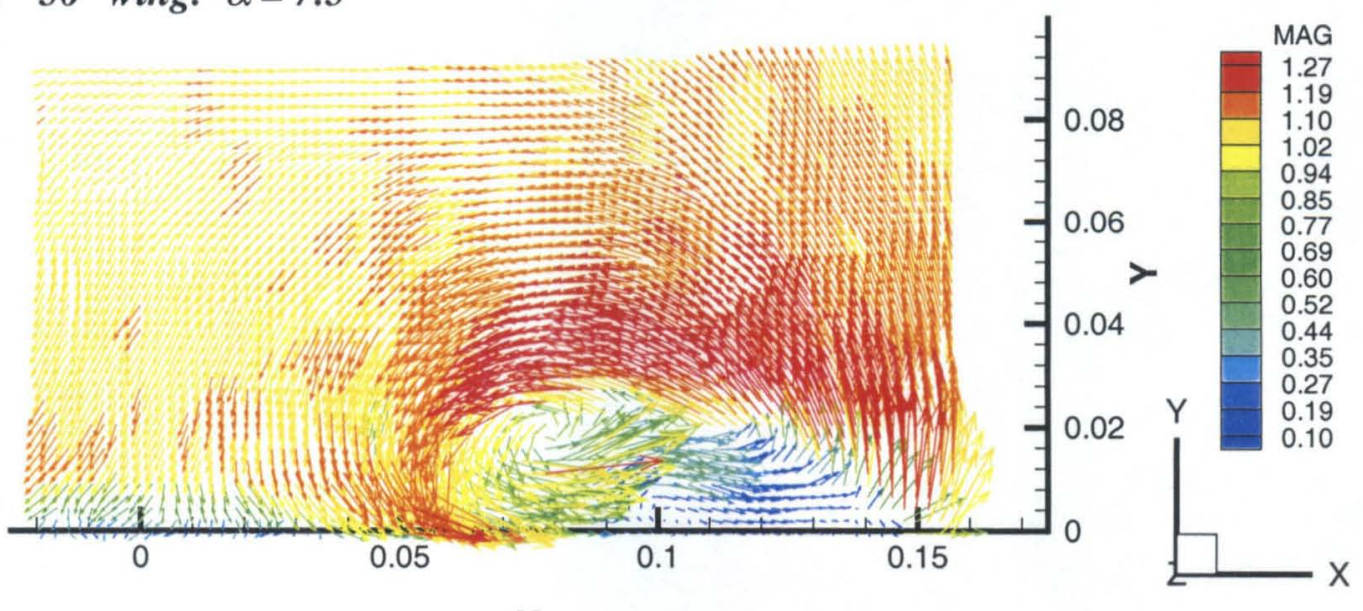

$\mathbf{X}$

Figure 5.39. $50^{\circ}$ wing, $\alpha=7.5^{\circ}$, instantaneous velocity field
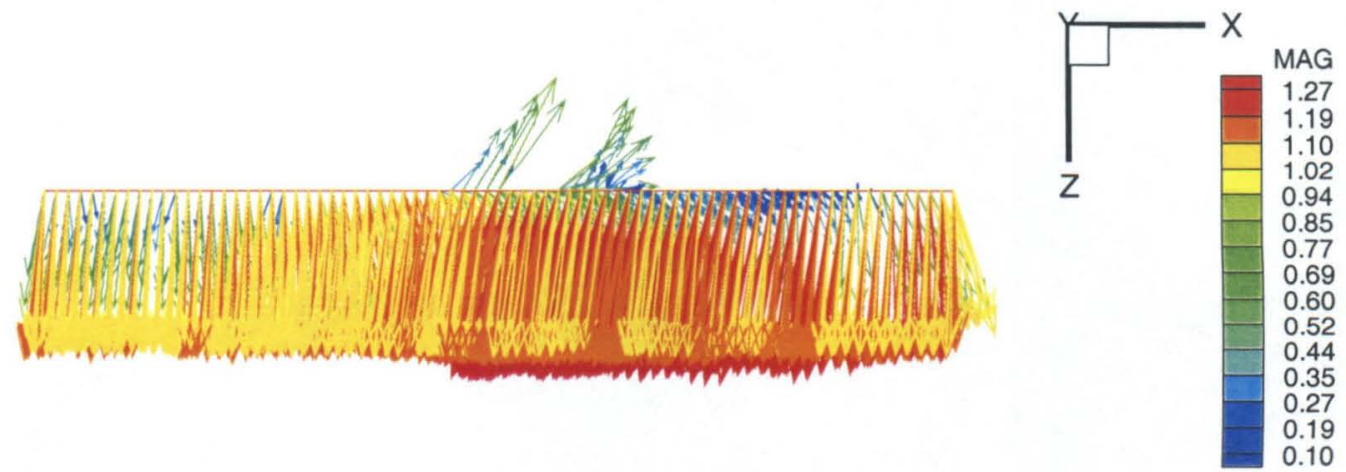

Figure 5.40. $50^{\circ}$ wing, $\alpha=7.5^{\circ}$, instantaneous velocity field, top view 


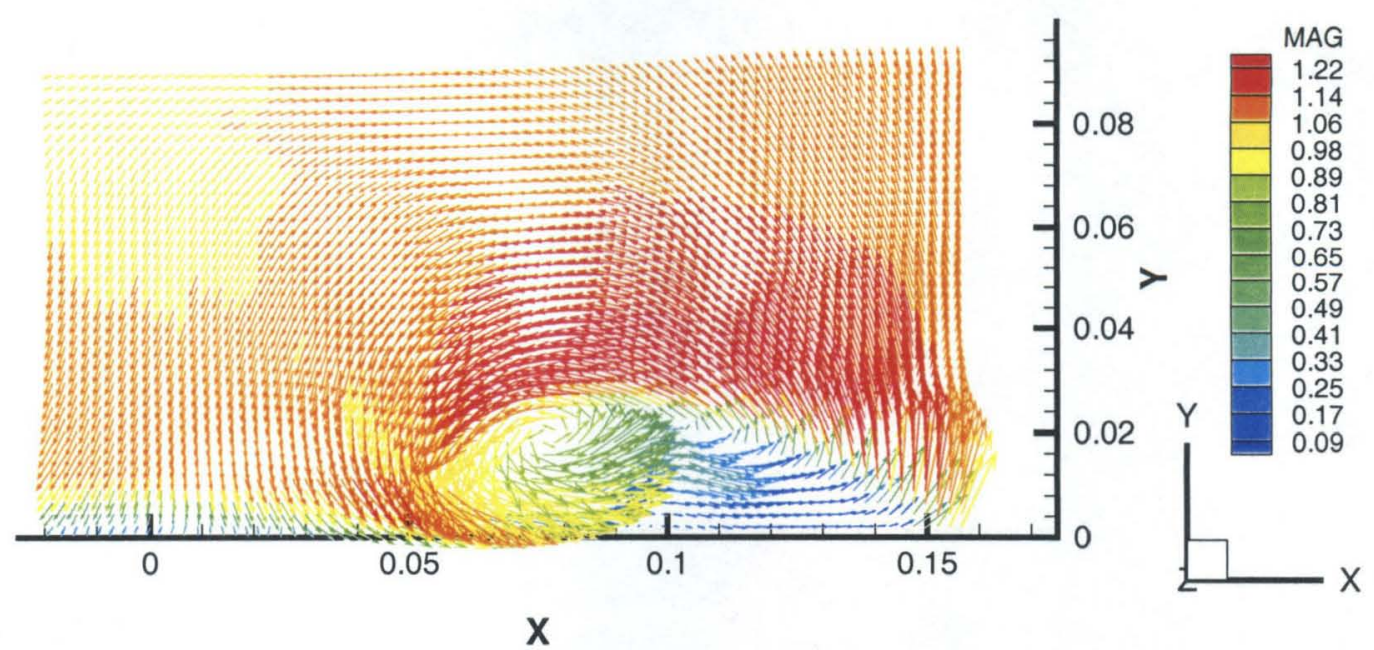

Figure 5.41. $50^{\circ}$ wing, $\alpha=7.5^{\circ}$, mean velocity field
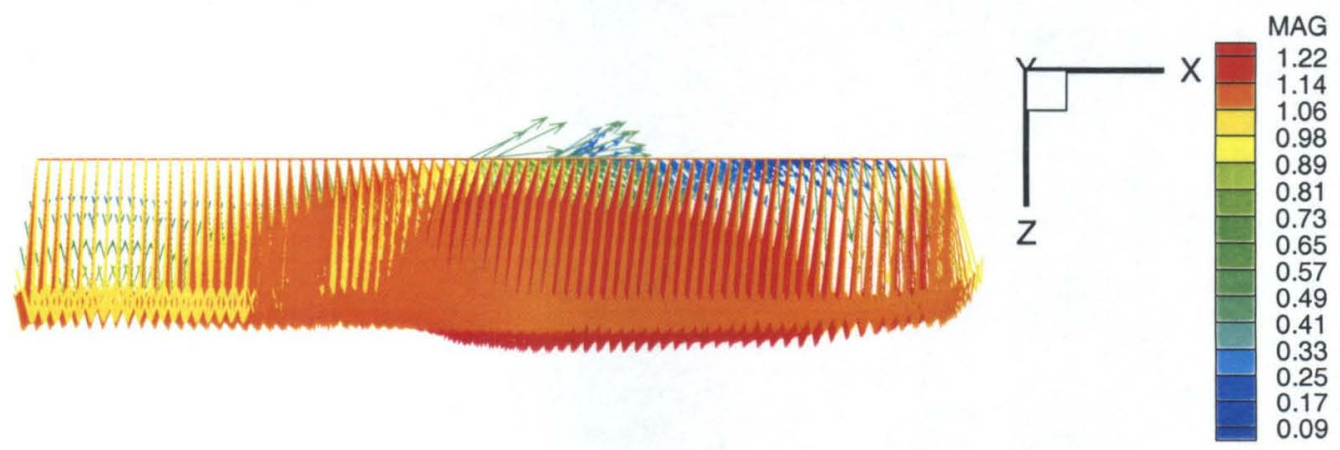

Figure 5.42. $50^{\circ}$ wing, $\alpha=7.5^{\circ}$, mean velocity field, top view

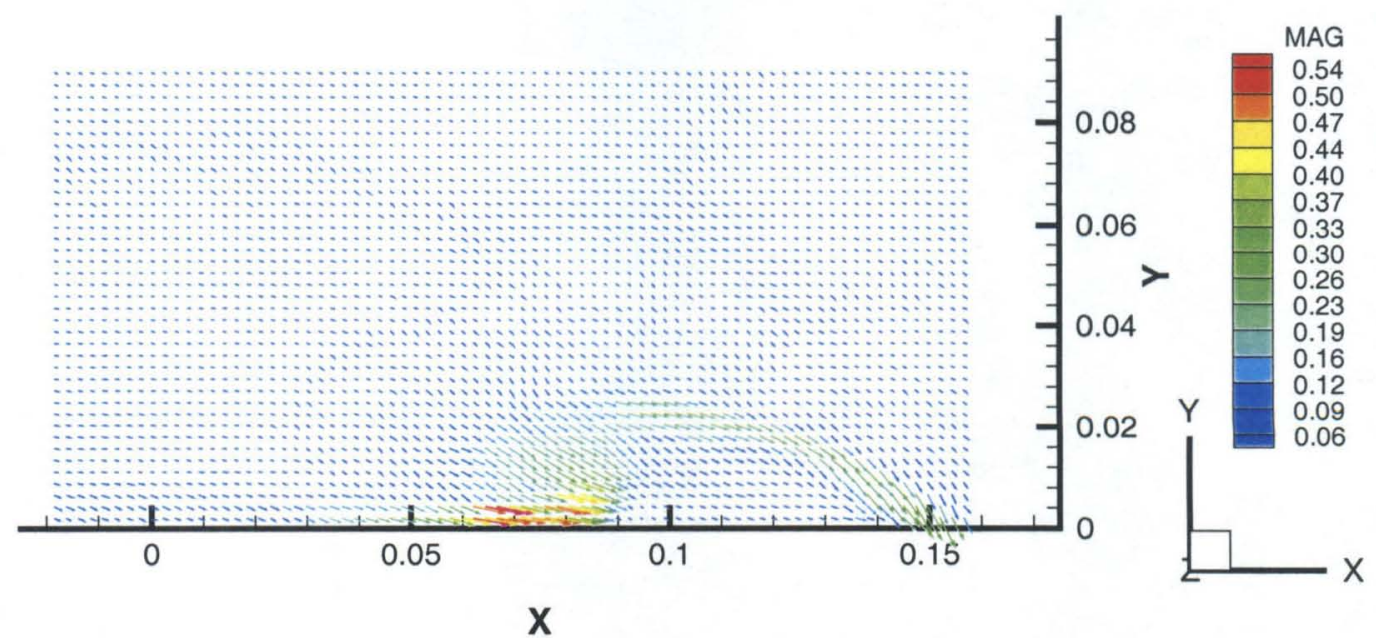

Figure 5.43. $50^{\circ}$ wing, $\alpha=7.5^{\circ}$, rms velocity field 
Evidence of a secondary vortex is now stronger. However, the primary vortex remains flattened to the wing leeward surface, as in the $5^{\circ}$ case. Unsteadiness is actually somewhat less than in the $5^{\circ}$ case. In both cases, velocity inside the core is of lower magnitude that in the outer fringes of the shear layer, or even of the free stream. This is in direct contrast to the $65^{\circ}$ wing.

\subsubsection{3 $50^{\circ}$ wing. $\alpha=10^{\circ}$}

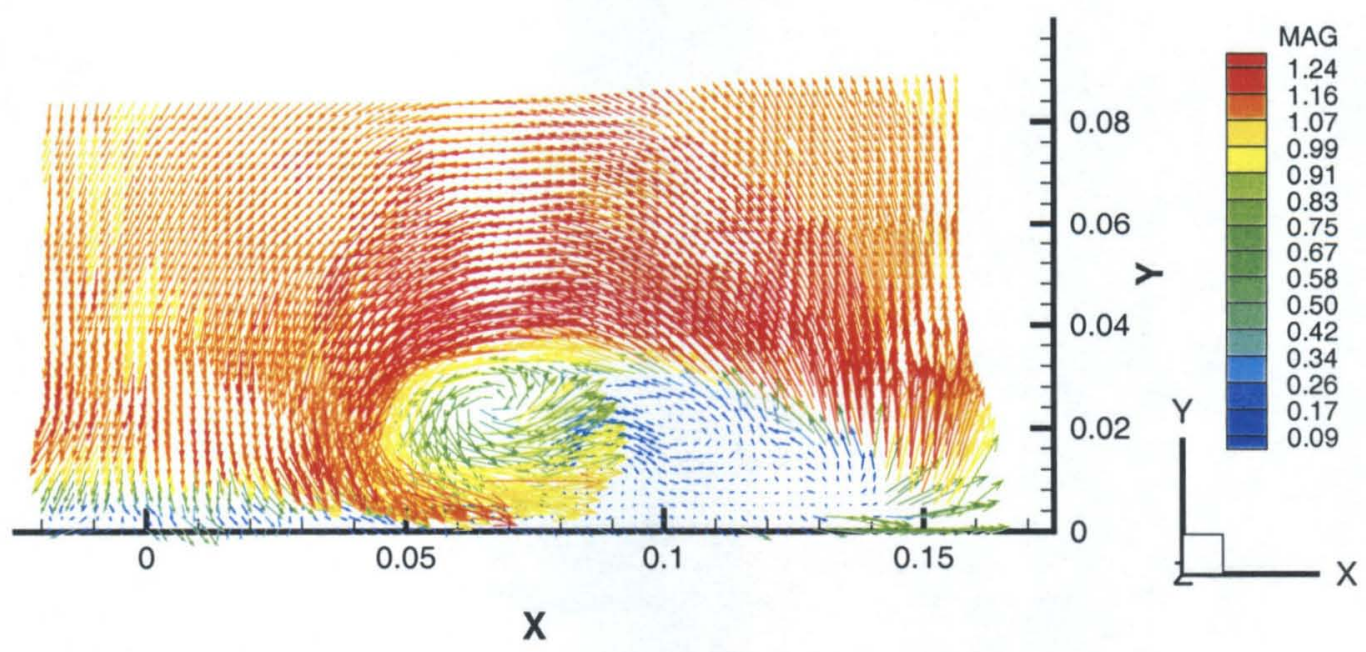

Figure 5.44. $50^{\circ}$ wing, $\alpha=10^{\circ}$, instantaneous velocity field

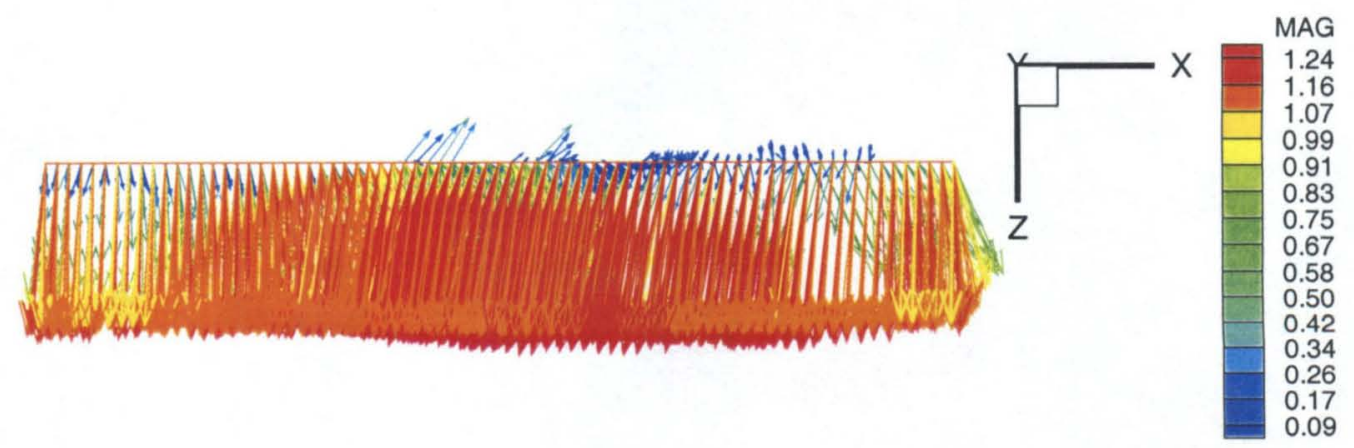

Figure 5.45. $50^{\circ}$ wing, $\alpha=10^{\circ}$, instantaneous velocity field, top view 


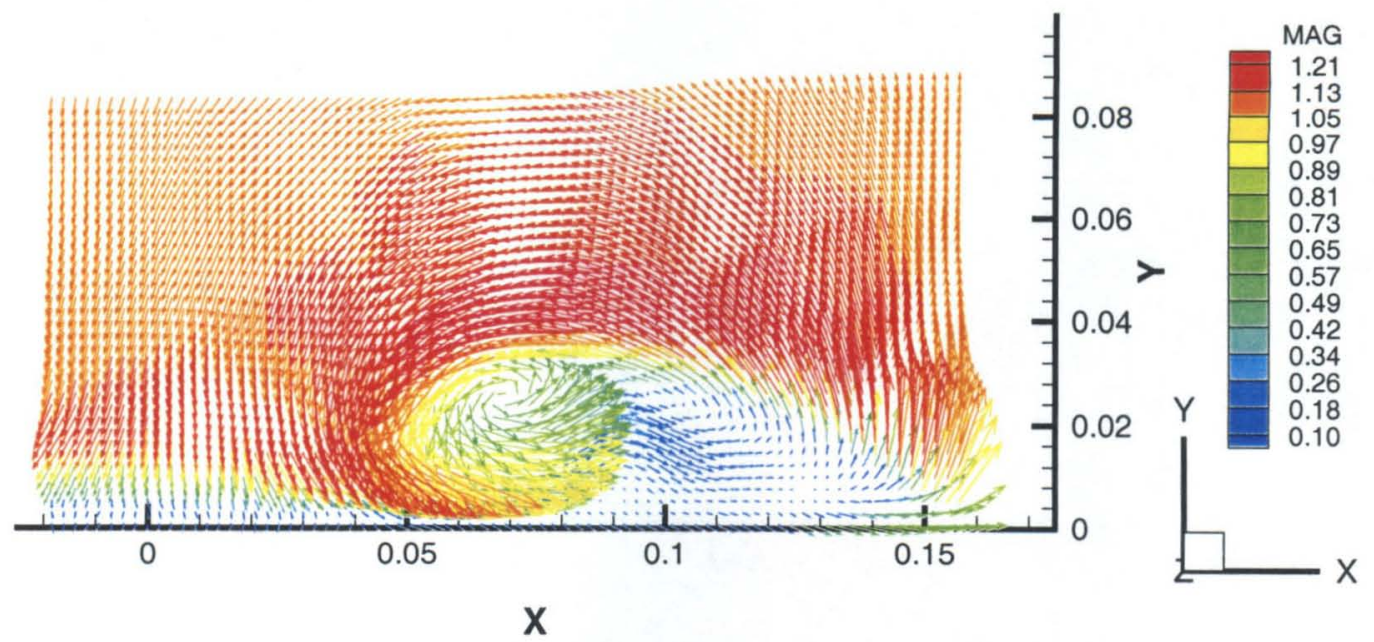

Figure 5.46. $50^{\circ}$ wing, $\alpha=10^{\circ}$, mean velocity field

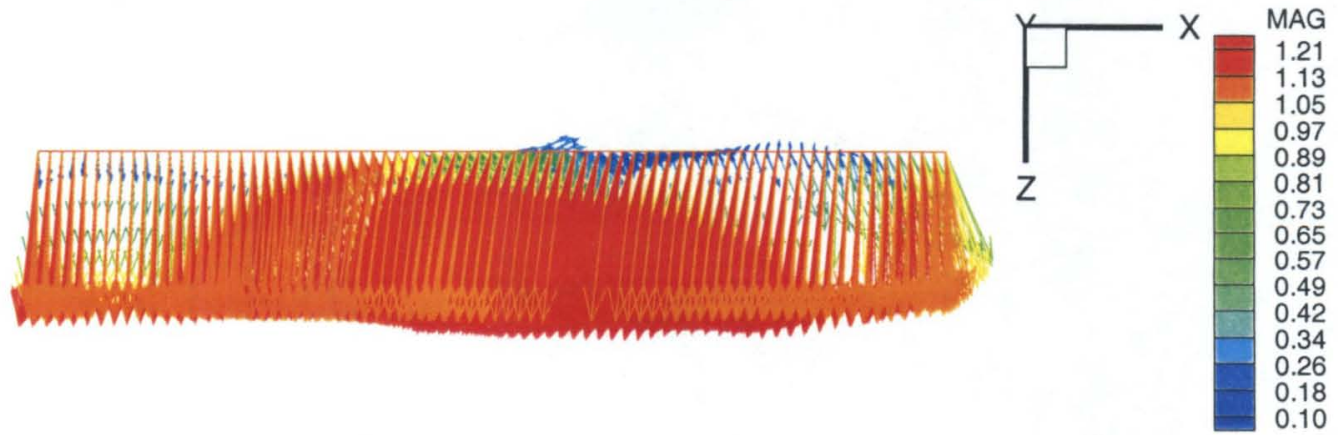

Figure 5.47. $50^{\circ}$ wing, $\alpha=10^{\circ}$, mean velocity field, top view

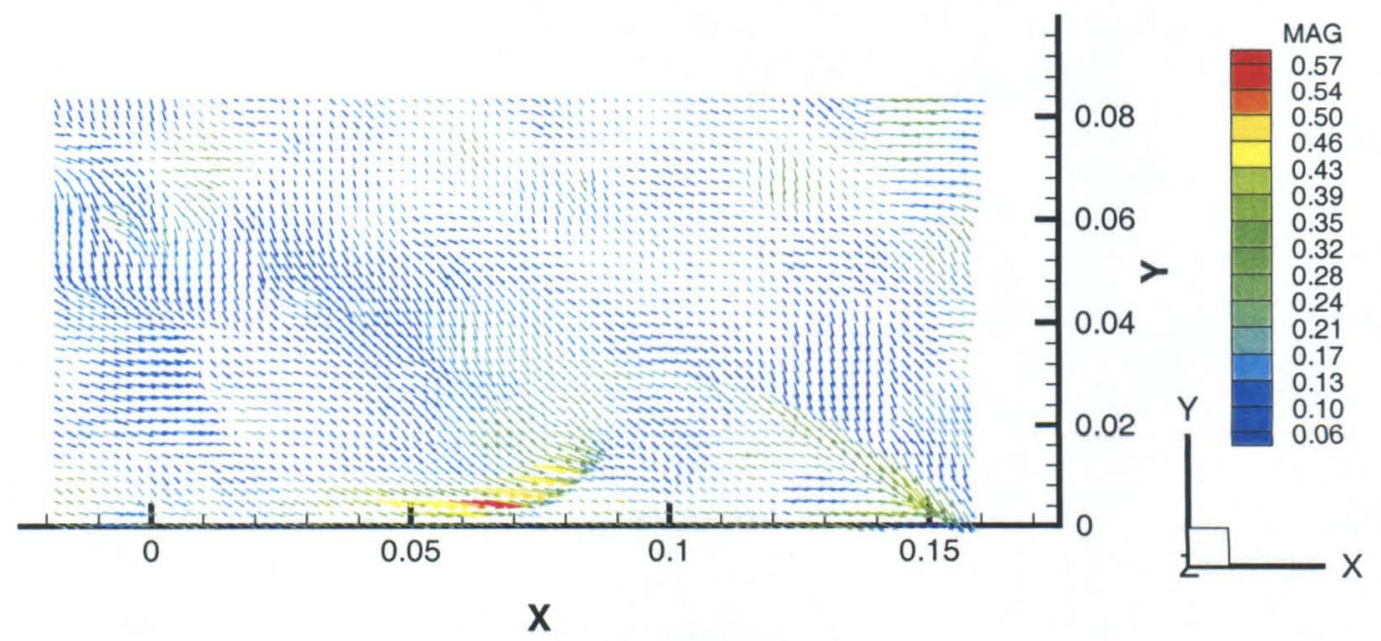

Figure 5.48. $50^{\circ}$ wing, $\alpha=10^{\circ}$, rms velocity field 
Again, the primary LEV remains flat. A stagnation zone is starting to appear outboard of the primary LEV. Velocity inside the primary LEV remains attenuated. Curiously, some pockets of unsteadiness are appearing outside of the shear layer, in nominally free stream flow. Unsteadiness of the rolled-up shear layer itself is actually lower than in the previous case.

\subsubsection{4 $50^{\circ}$ wing. $\alpha=12.5^{\circ}$}

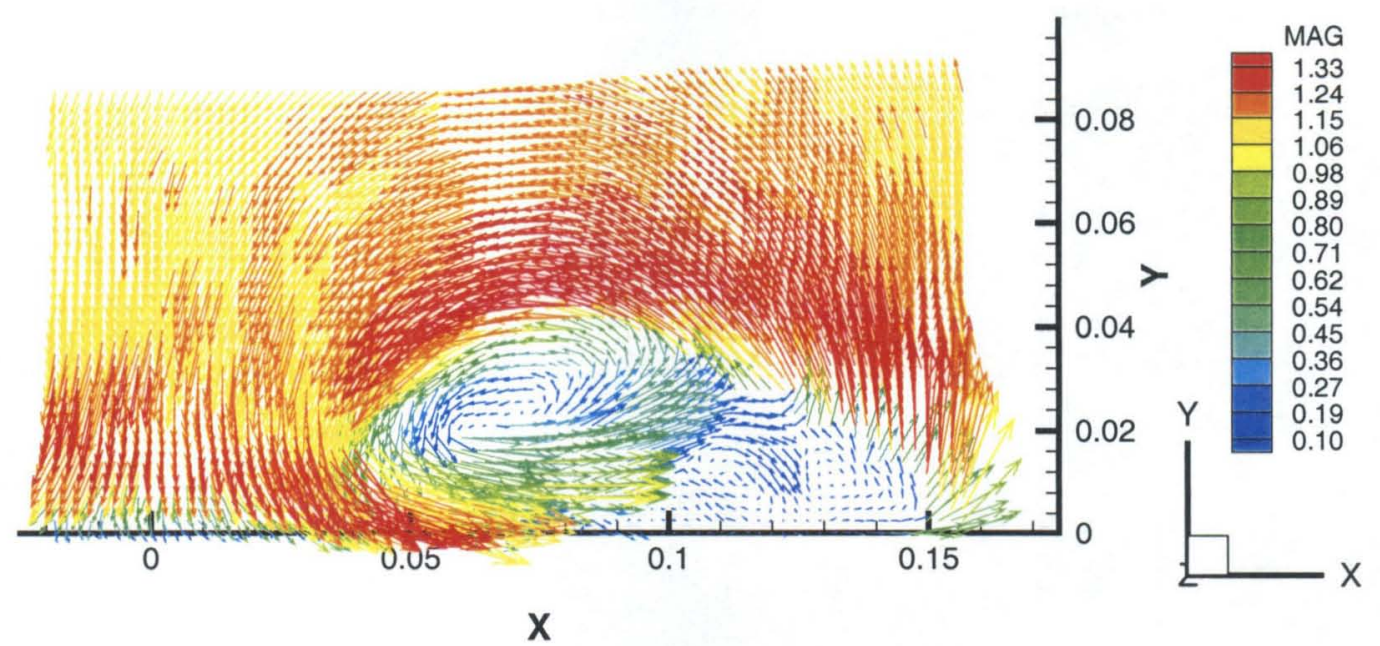

Figure 5.49. $50^{\circ}$ wing, $\alpha=12.5^{\circ}$, instantaneous velocity field
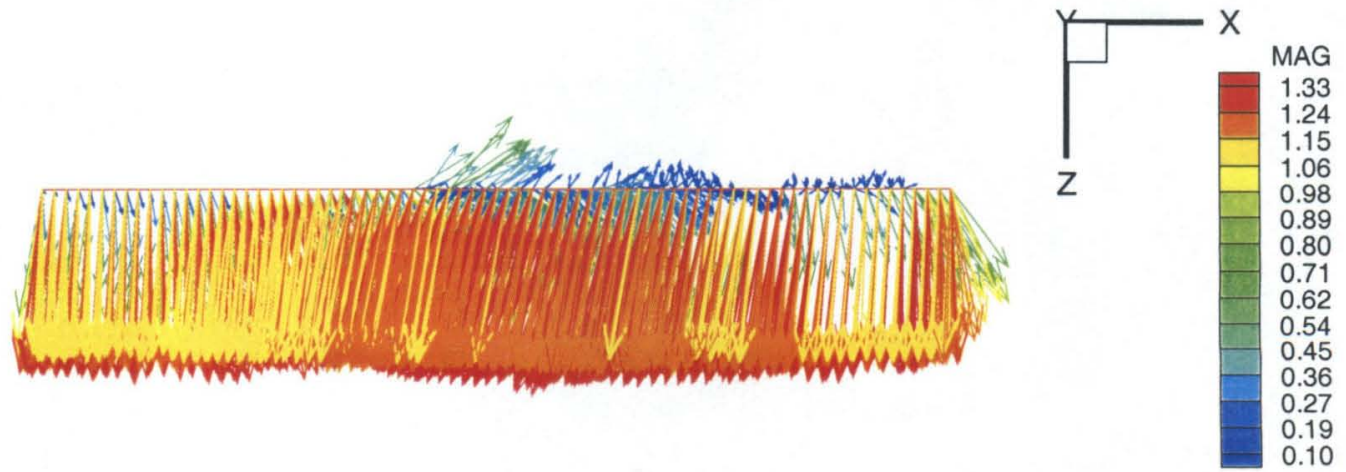

Figure 5.50. $50^{\circ}$ wing, $\alpha=12.5^{\circ}$, instantaneous velocity field, top view

Now the mean data are with respect to 250 instantaneous velocity records - and thus, $\sim 12.5$ convective times. 


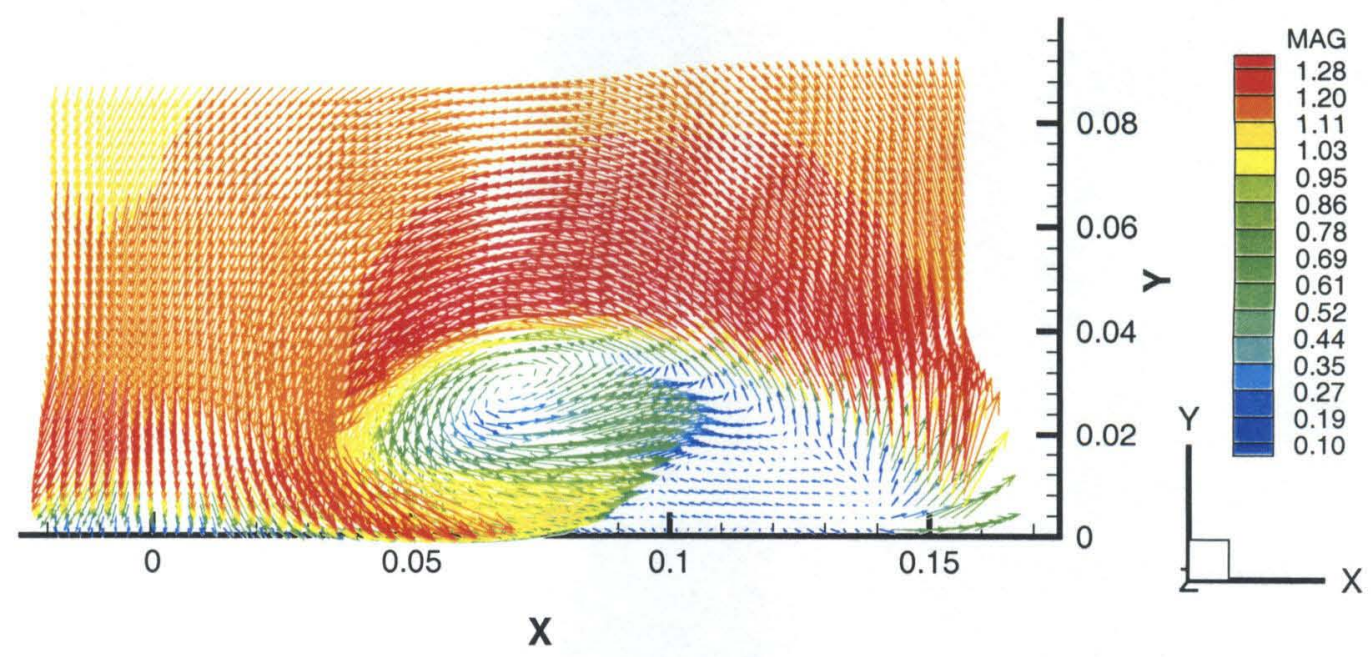

Figure 5.51. $50^{\circ}$ wing, $\alpha=12.5^{\circ}$, mean velocity field

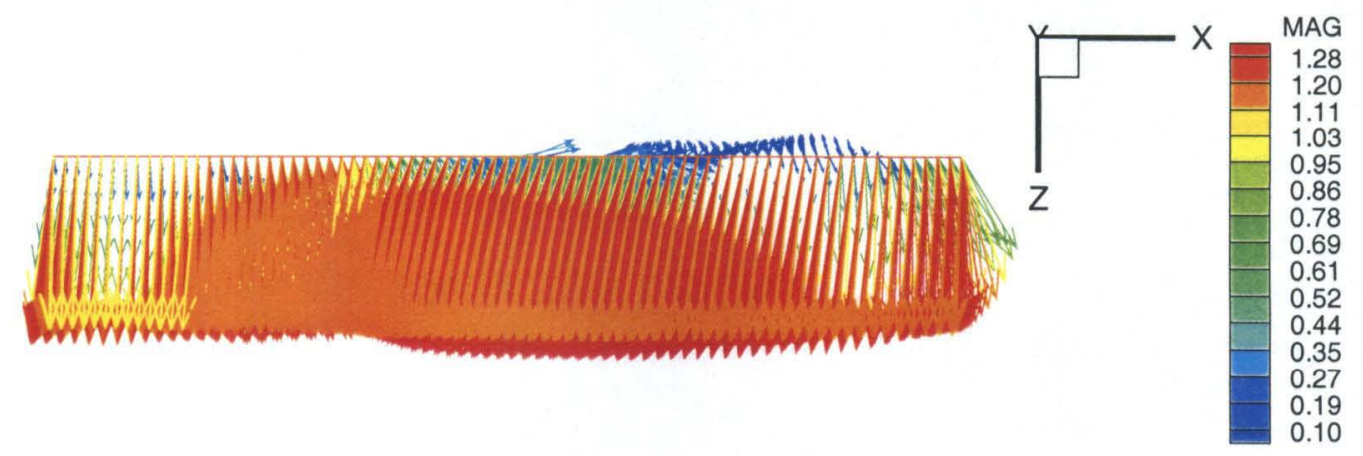

Figure 5.52. $50^{\circ}$ wing, $\alpha=12.5^{\circ}$, mean velocity field, top view

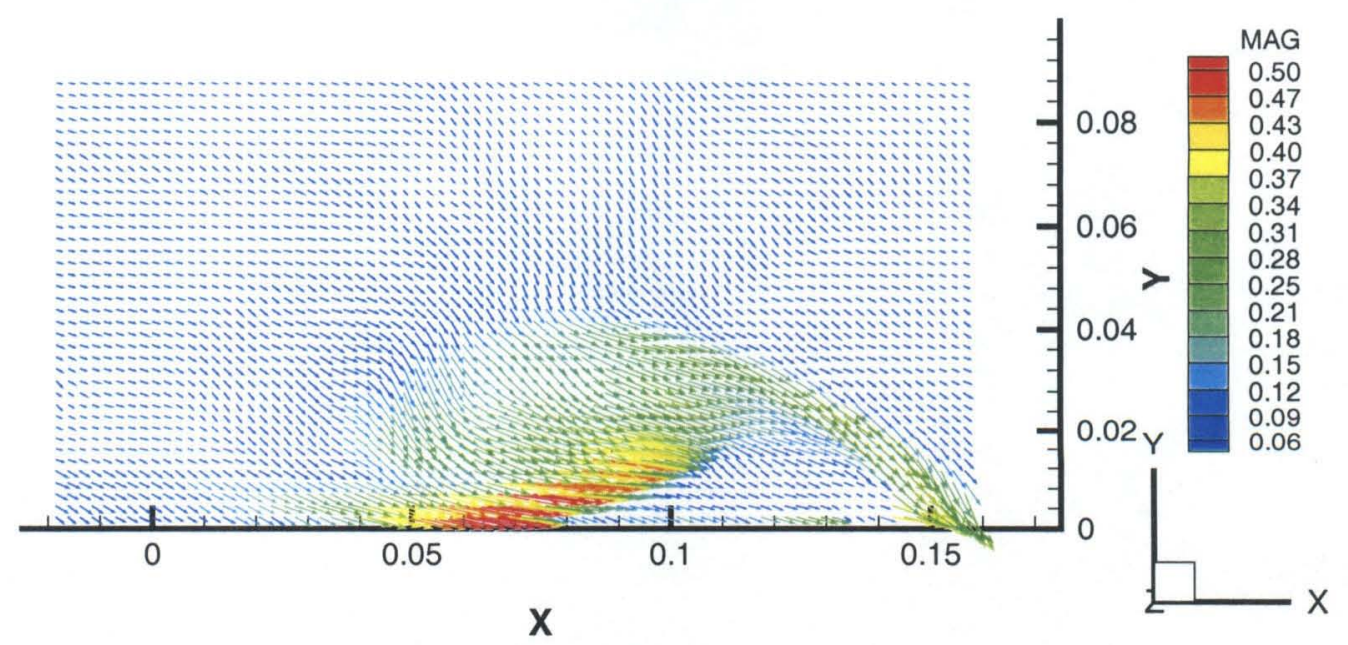

Figure 5.53. $50^{\circ}$ wing, $\alpha=12.5^{\circ}$, rms velocity field

The principal distinction between this and all prior cases - for either wing - is the sharp increase in unsteadiness. This is in complete agreement with the flow visualization 
results, where LEVs displayed appreciable streamwise undulations. The shear layer and the entire primary LEV region are subject to fairly strong velocity variations, although the position of the shear layer remains fairly constant. The stagnation outboard of the main LEV is not appreciably from the previous cases. It also differs little in going from the instantaneous case to the mean. This suggests that outboard of the primary LEV, the very low speed flow is not punctuated by sporadic eruptions. At the core of the primary vortex, flow is almost stagnant. This is again in good agreement with the flow visualization (Figure 4.59).

5.3.2.5 50 ${ }^{\circ}$ wing. $\alpha=15^{\circ}$

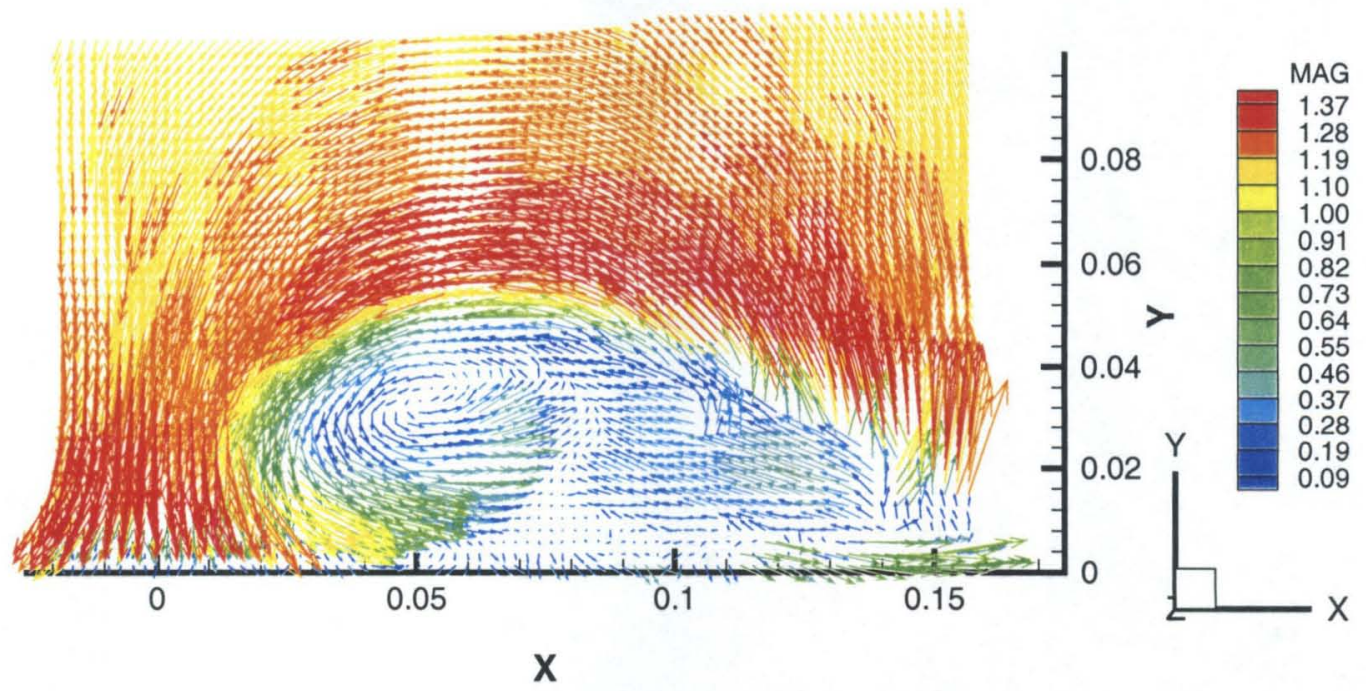

Figure 5.54. 50 $^{\circ}$ wing, $\alpha=15^{\circ}$, instantaneous velocity field 


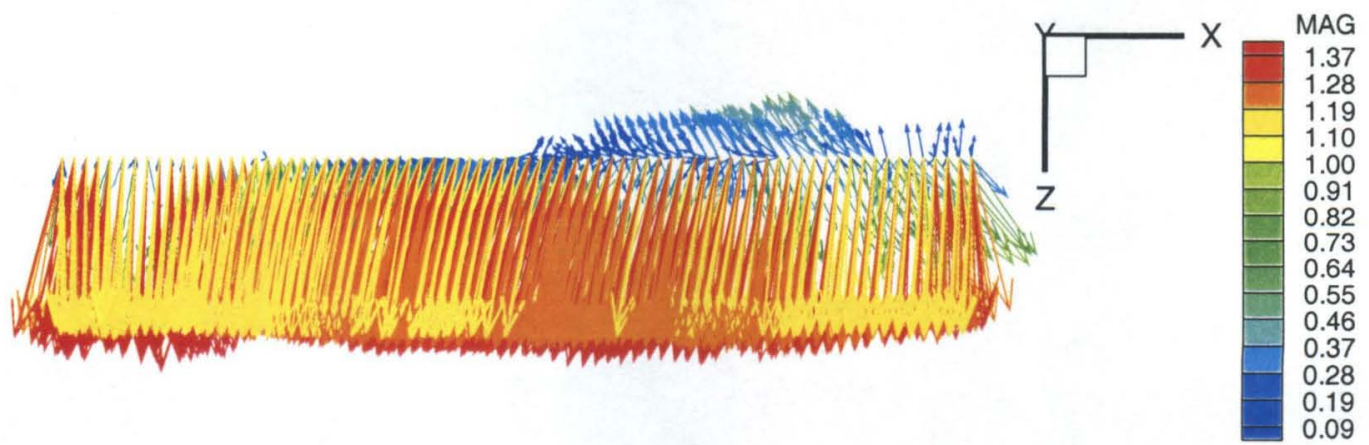

Figure 5.55. $50^{\circ}$ wing, $\alpha=15^{\circ}$, instantaneous velocity field, top view

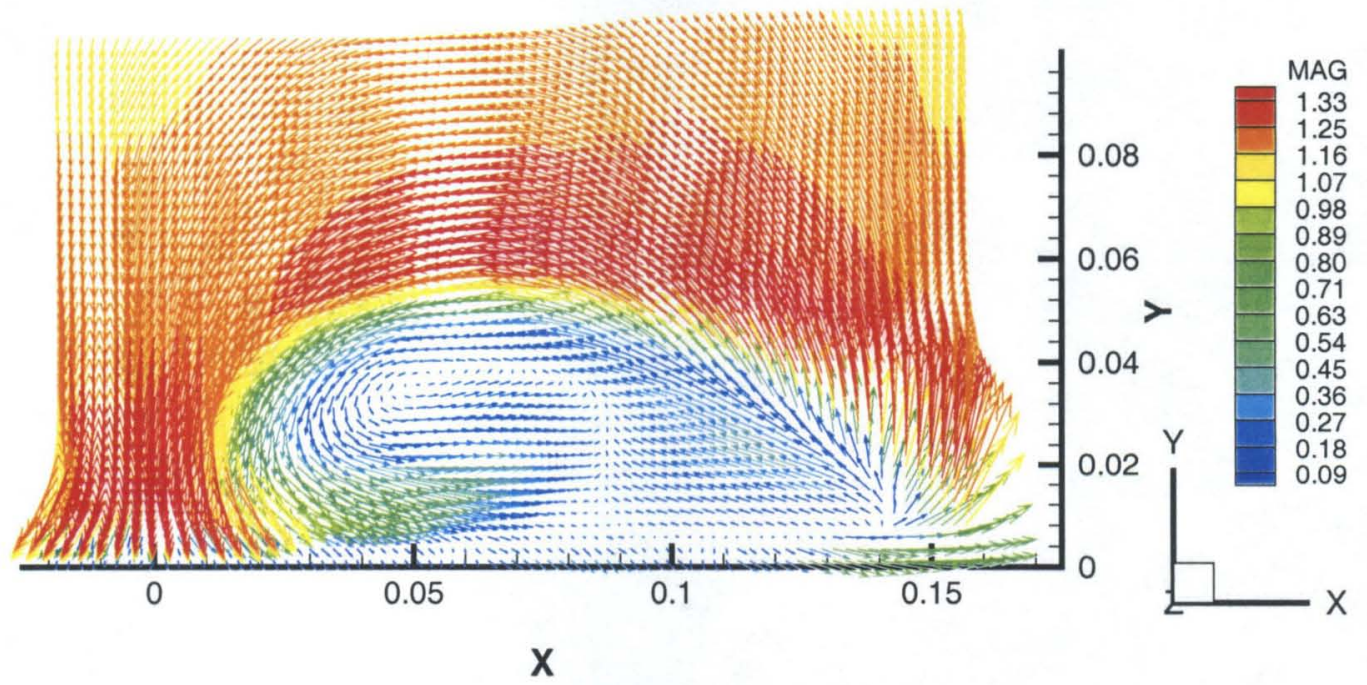

Figure 5.56. $50^{\circ}$ wing, $\alpha=15^{\circ}$, mean velocity field

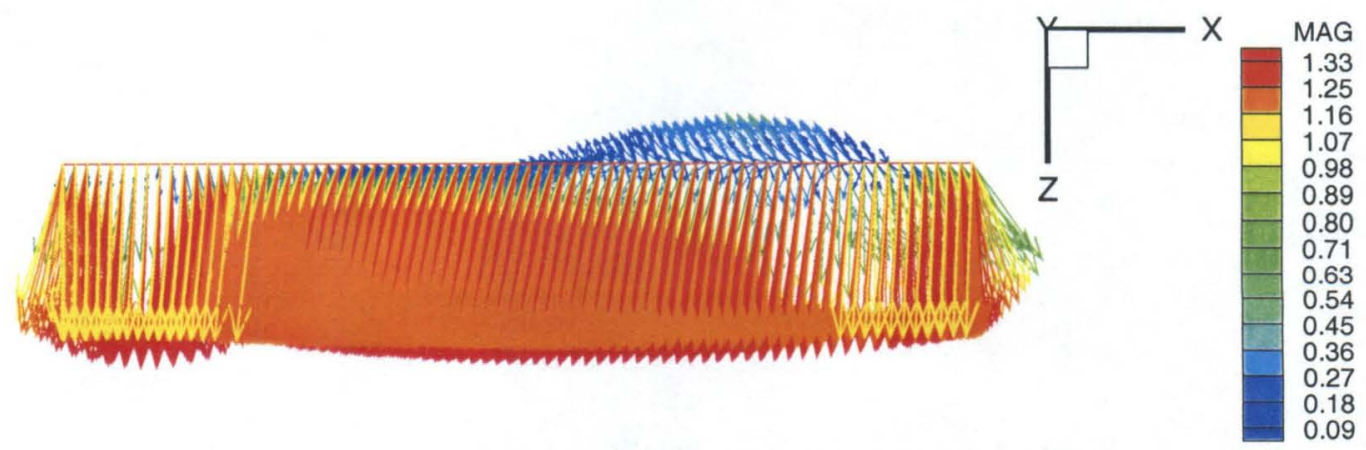

Figure 5.57. $50^{\circ}$ wing, $\alpha=15^{\circ}$, mean velocity field, top view 


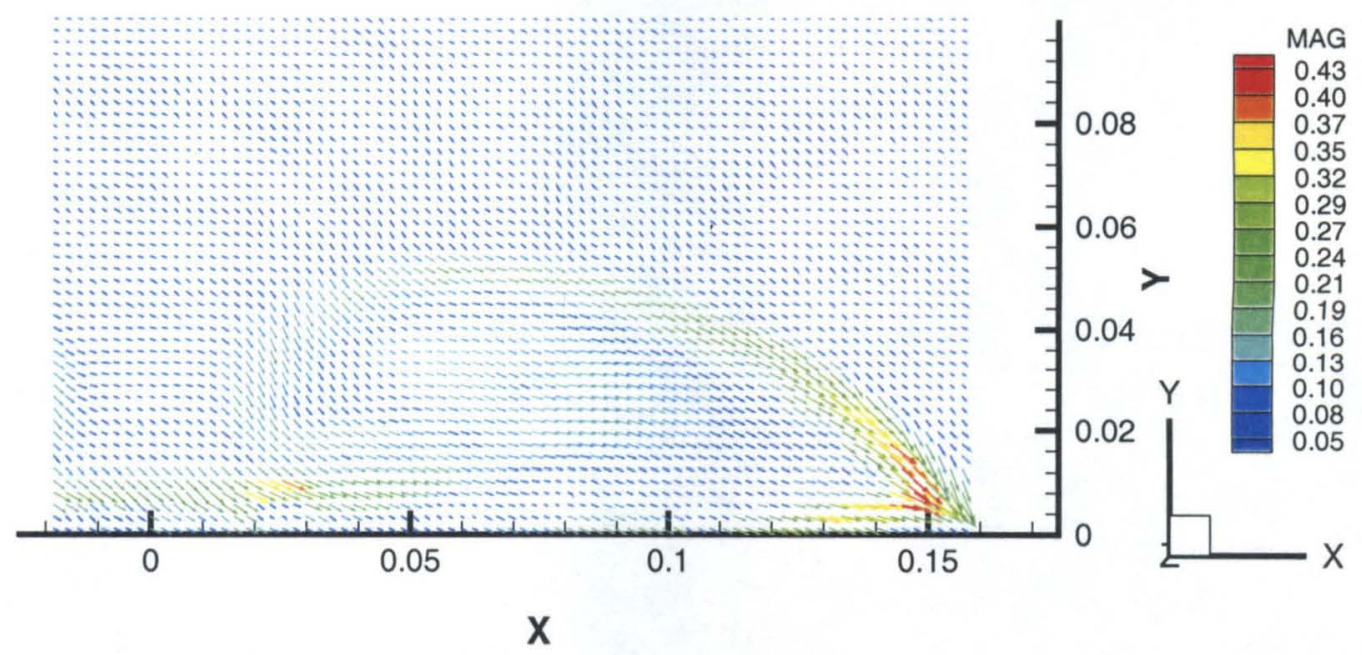

Figure 5.58. $50^{\circ}$ wing, $\alpha=15^{\circ}$, rms velocity field

This angle of attack may be viewed as the true onset of vortex breakdown; or rather, as the angle at which $\mathrm{VB}$ crosses the $\mathrm{z} / \mathrm{c}=0.296$ streamwise station. A coherent primary LEV really no longer exists. But the leading edge shear layer maintains its rolled-up geometry, all the way up to its "attachment" line on the leeward surface. Indeed, the shear layer bounds a region of largely separated, low-speed flow. In the outboard region, it is seen that apparent stagnation has transitioned to flow reversal. The rms plot shows comparatively minor unsteadiness.

\subsubsection{6 $50^{\circ}$ wing. $\alpha=20^{\circ}$}

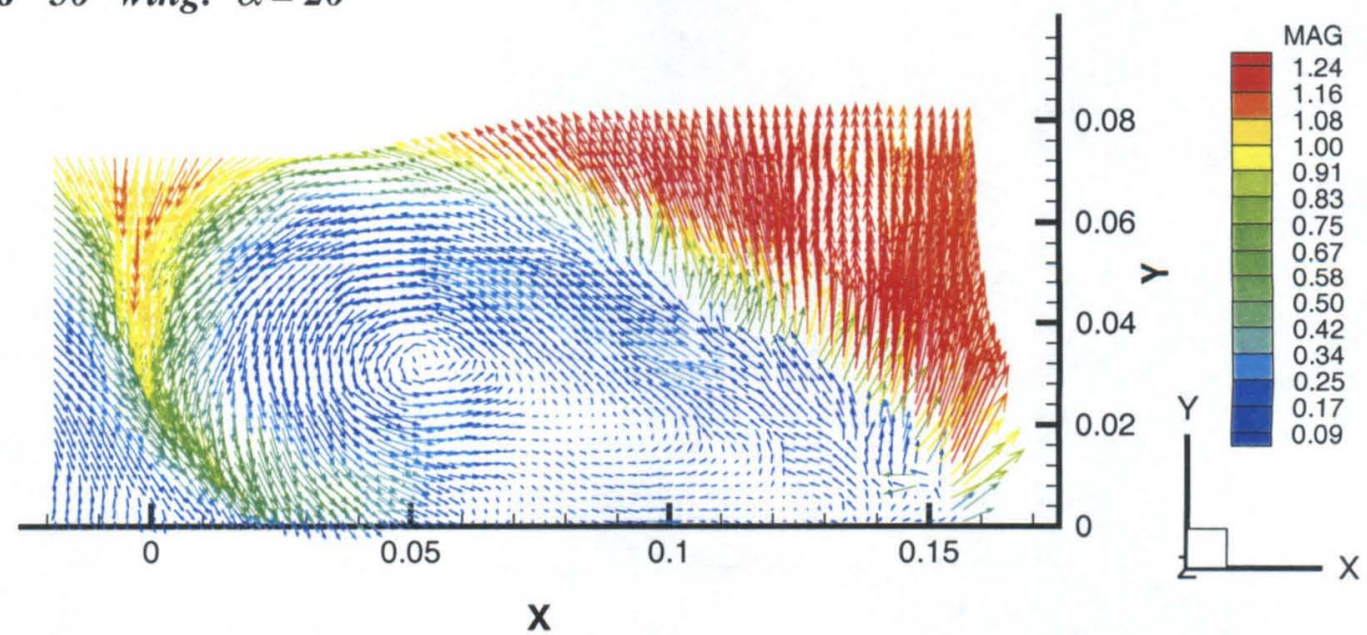

Figure 5.59. $50^{\circ}$ wing, $\alpha=20^{\circ}$, instantaneous velocity field 


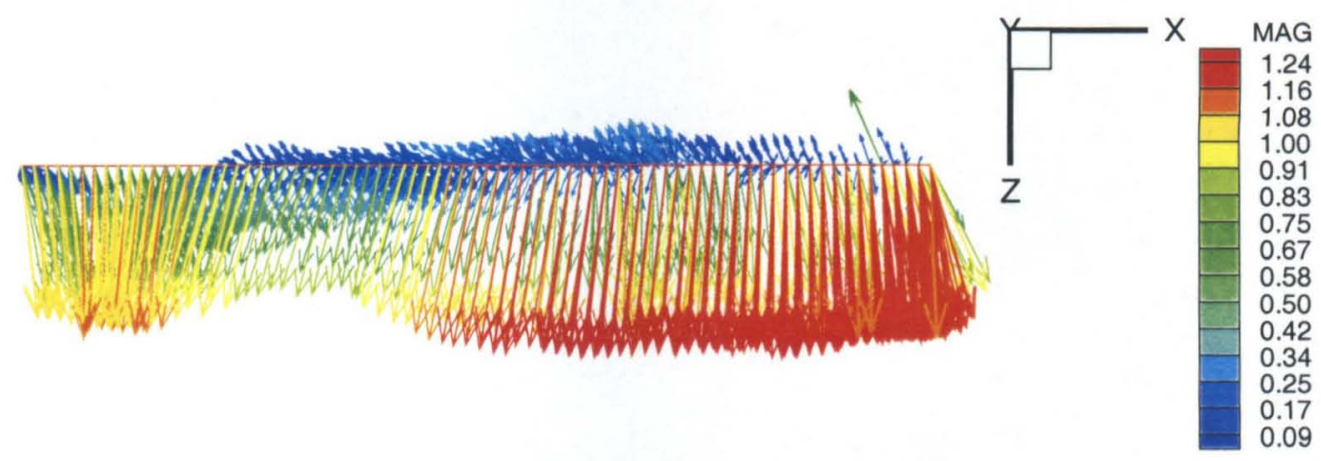

Figure 5.60. $50^{\circ}$ wing, $\alpha=20^{\circ}$, instantaneous velocity field, top view

100 frames (5 convective times later), the instantaneous velocity image now appears to have less organized rotational motion bounded by the shear layer (Figure 5.61).

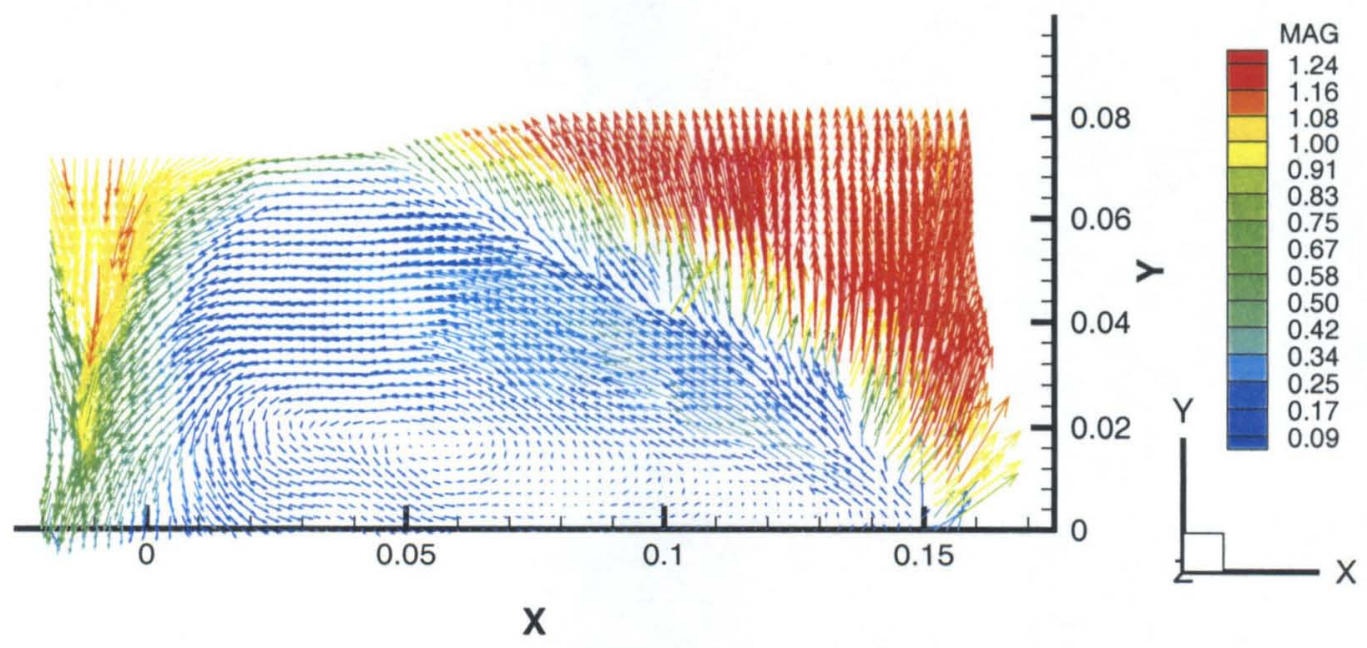

Figure 5.61. $50^{\circ}$ wing, $\alpha=20^{\circ}$, instantaneous velocity field, another sample

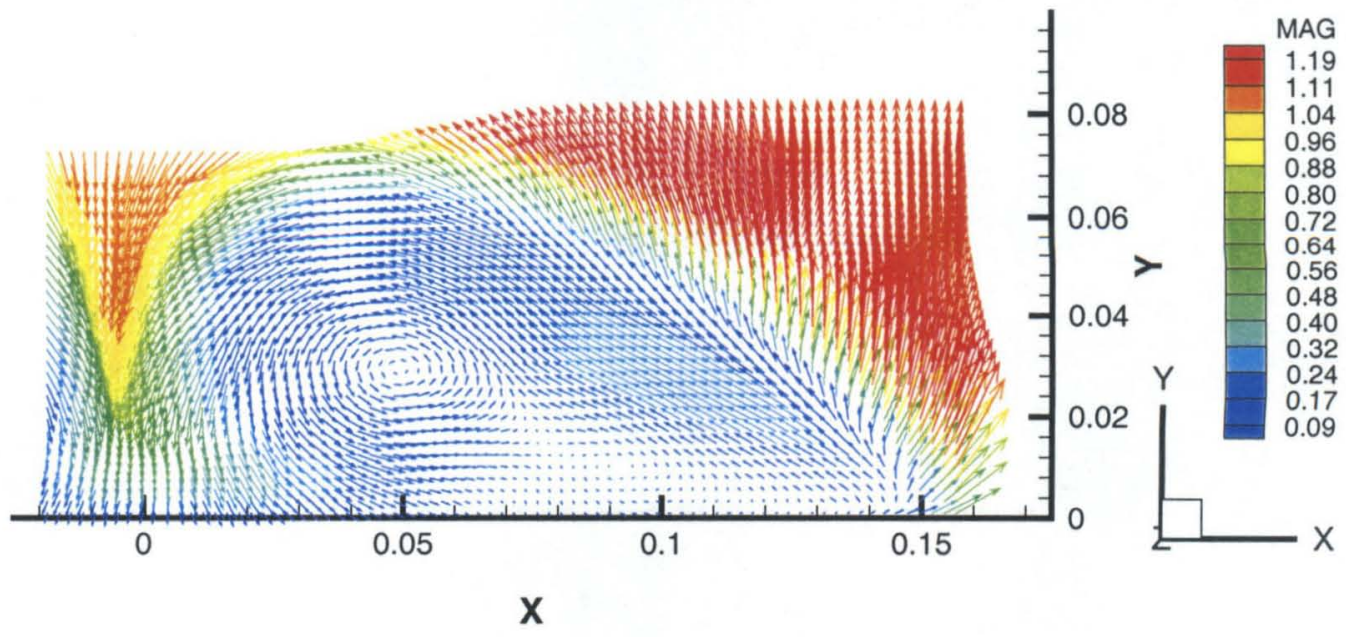

Figure 5.62. $50^{\circ}$ wing, $\alpha=20^{\circ}$, mean velocity field 


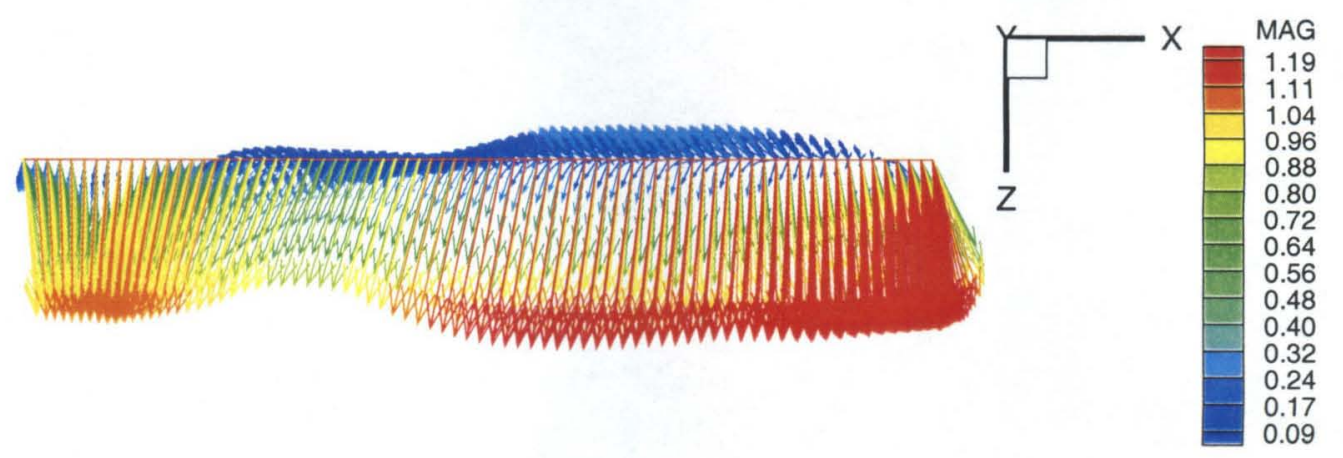

Figure 5.63. $50^{\circ}$ wing, $\alpha=20^{\circ}$, mean velocity field, top view

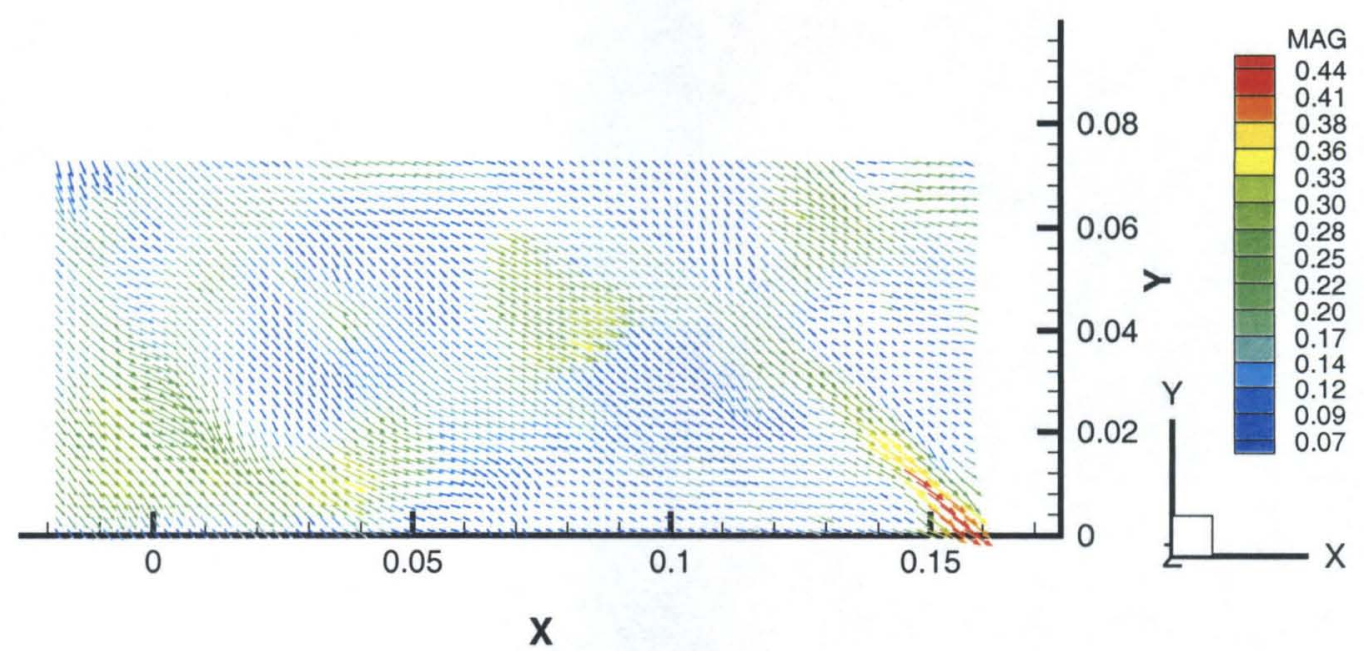

Figure 5.64. $50^{\circ}$ wing, $\alpha=20^{\circ}$, rms velocity field

For the $20^{\circ}$ angle of attack, the trend seen at $15^{\circ}$ is amplified. The shear layer still rolls up in a coherent fashion, though even the flow at the wing centerline, heretofore fully attached, is showing signs of eventual separation. That would probably occur at around $22-25^{\circ}$ angle of attack, whence the leading edge shear layers would coalesce into one "bubble" of separation blanketing the wing.

There is evidence of some residual rotational motion inside the region bounded by the shear layer, but essentially, the flow has stagnation, and "coherent separation" in the language of Chapter 1 is lost. A "wake-like" axial velocity profile dominates what was 
the primary LEV core region. Unsteadiness is again weaker than in the seminal $12.5^{\circ}$ case.

\subsection{Streamline projection sketches}

Projecting the velocity vector plots onto two dimensions, and constructing curves of tangents to the projected velocity vectors, one obtains projected 2-D "streamlines." As pointed out, e.g., by Delery ${ }^{72}$, these are not true streamlines, but they do provide some qualitative insight into the flow pattern. These data are of course obtainable with regular 2-D PIV. In what follows, such streamlines are shown superimposed on the mean velocity vector plots of Section 5.3. Figures are denoted by wing sweep angle and angle of attack.

It is interesting to compare conclusions from the velocity vector plots vs. the projected streamline plots regarding the presence of the primary and secondary vortices. In particular, only the $50^{\circ}$ wing, $12.5^{\circ}$ angle of attack case is seen to exhibit a definite secondary vortex structure. Yet at this angle of attack, no such structures were seen in the flow visualization.

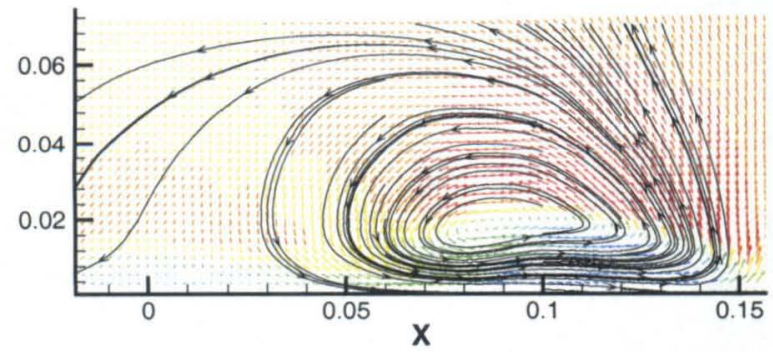

Figure 5.65. $50^{\circ}$ wing, $5^{\circ} \mathrm{AOA}$

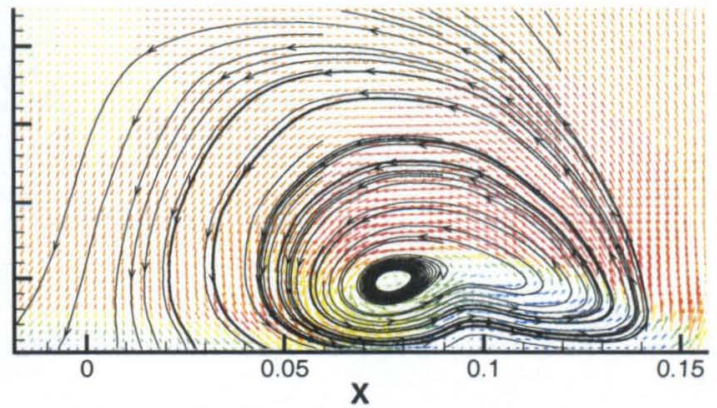

Figure 5.66. $50^{\circ}$ wing, $7.5^{\circ} \mathrm{AOA}$ 


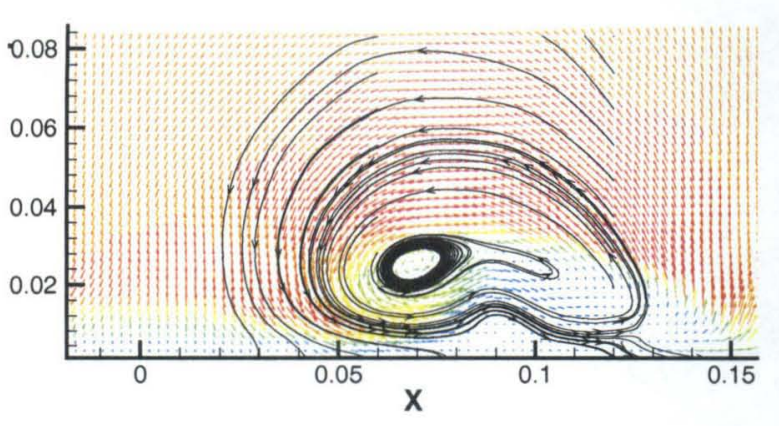

Figure 5.67. $50^{\circ}$ wing, $10^{\circ} \mathrm{AOA}$

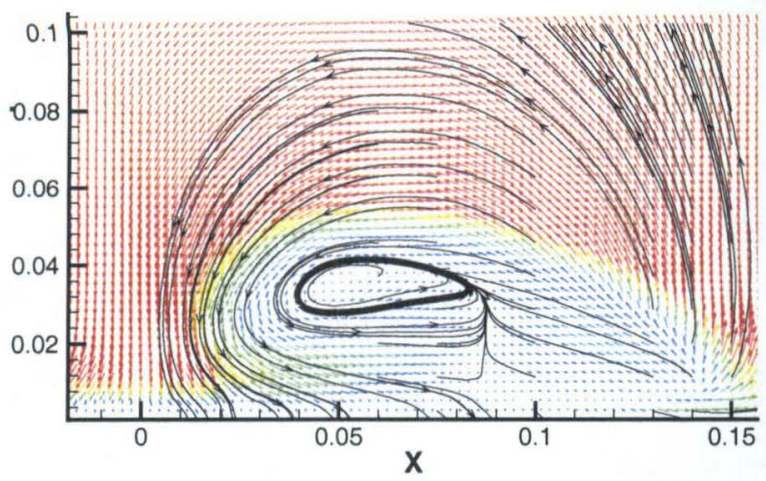

Figure 5.69. $50^{\circ}$ wing, $15^{\circ} \mathrm{AOA}$

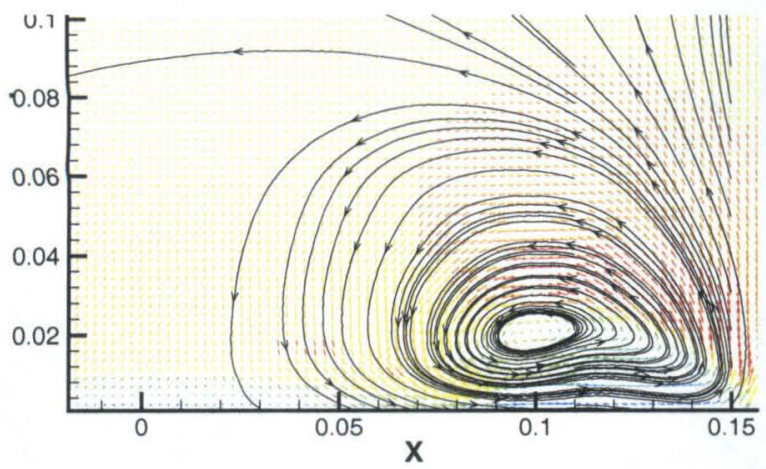

Figure 5.71. $65^{\circ}$ wing, $5^{\circ} \mathrm{AOA}$

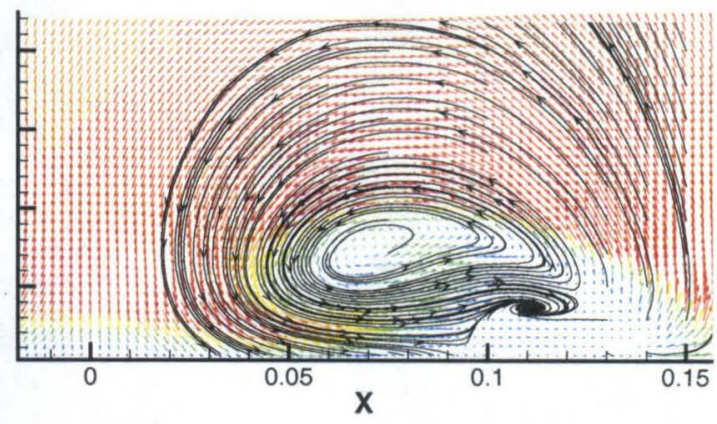

Figure 5.68. $5^{\circ}$ wing, $12.5^{\circ} \mathrm{AOA}$

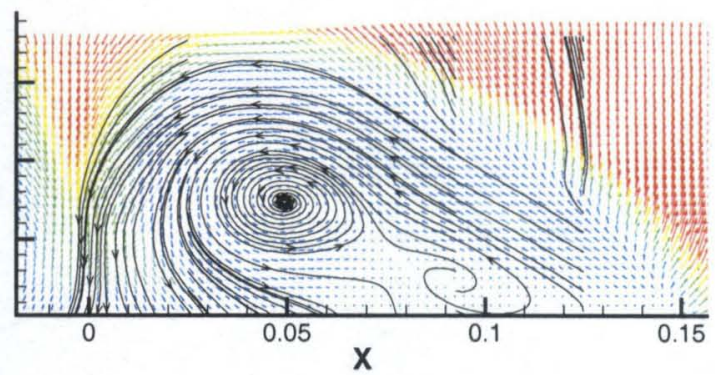

Figure 5.70. $50^{\circ}$ wing, $20^{\circ}$ AOA

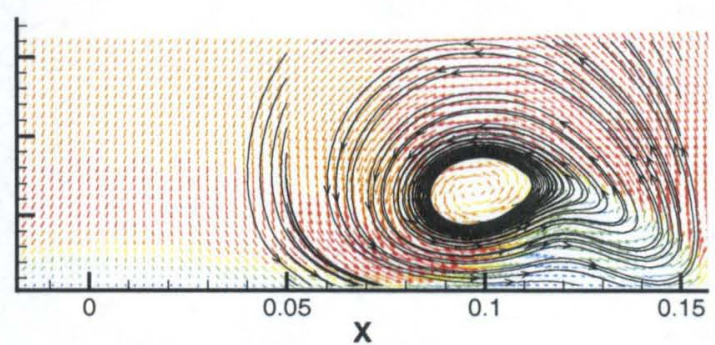

Figure 5.72. $65^{\circ}$ wing, $7.5^{\circ} \mathrm{AOA}$ 


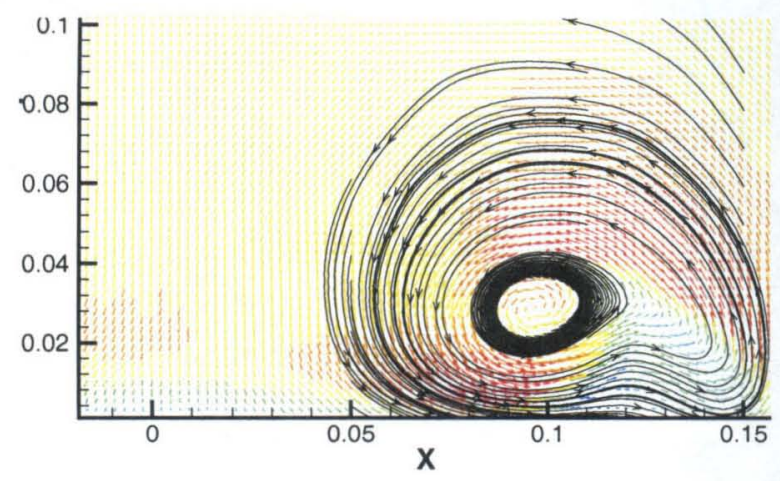

Figure 5.73. $65^{\circ}$ wing, $10^{\circ} \mathrm{AOA}$

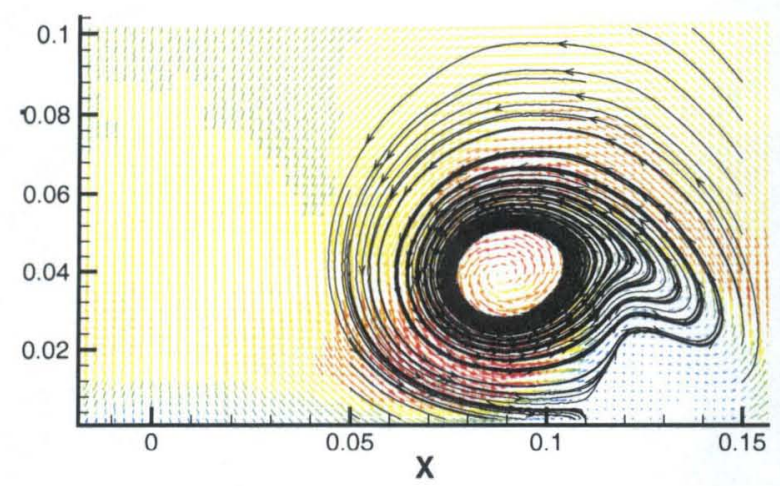

Figure 5.75. $65^{\circ}$ wing, $15^{\circ} \mathrm{AOA}$

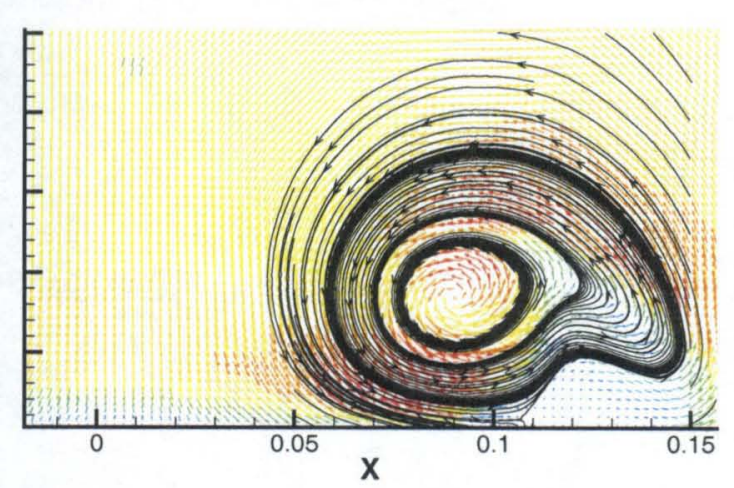

Figure 5.74. $65^{\circ}$ wing, $12.5^{\circ} \mathrm{AOA}$

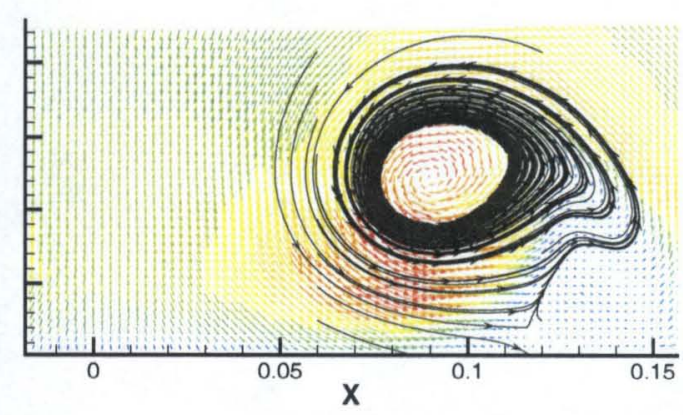

Figure 5.76. $65^{\circ}$ wing, $20^{\circ} \mathrm{AOA}$

Nearly all of the streamline plots show an "elongated" orbit about what it apparently the primary LEV axis, with a "tail" extending outboard. Intuitively, the secondary vortex should lie underneath this tail. This is indeed the case for the $50^{\circ}$ wing at $12.5^{\circ}$ angle of attack.

For the $50^{\circ}$ wing, a vortex-like projected streamline pattern remains even out to $20^{\circ}$ angle of attack. The pattern is illustrative of what may be regarded at the LEV core axis, but says nothing about vortical strength. There is even a minor apparent "rotating" motion in the outboard stagnation region. However, the comment regarding potentially misleading projected streamlines should be noted. 
For the $65^{\circ}$ wing, it is seen that from $12.5^{\circ}$ angle of attack onwards, the outboard stagnation region is unmistakable.

It should also be noted that how much the streamlines appear to fill in the primary LEV core strongly depends on the location of the streamline "rake." The closer that a rake happens to be to the core axis, the more filled in the core will be with streamlines. Since radial velocity in the LEV is very small, compared to the other two components, projected streamlines circling the LEV core can superficially resemble limit cycles. Proper choice of rake placement is a matter of taste and opinion.

\subsection{Vorticity plots}

The following resume of vorticity plots follows the format of Section 5.3. Contours of axial vorticity are given in streamwise cuts. The scope of these data is essentially identical to what one would have obtained from classical 2-D PIV. Regions of high positive and negative vorticity are generally present in all data sets. The intermediate range of near-zero vorticity is doubtless heavily contaminated by noise, either due to the numerical noise of differentiating discrete data, or of the general amplification of errors in PIV. Whereas it is clear from the velocity vector plots that the primary LEV core will be a region of strong axial vorticity, at least in crossflow planes upstream of breakdown, small regions of concentrated vorticity ("sub-structures"), such as those observed by Shih and Ding ${ }^{38}$, Gad-el-Haq and Blackwelder ${ }^{73}$, and others, can not be observed from the velocity plots alone. These were, however, clearly visible in the rolling-up shear layer and in the leeward surface boundary layer, especially where the latter is close to the primary LEV. 
The actual numerical values of vorticity are to be viewed with caution, since "peak" values strongly skew the entire image, and these peaks are easily affected by numerical noise and the windowing resolution of PIV. This is especially apparent at the wing leading edge region. It should also again be pointed out that the interrogation planes from which the out-of-plane vorticity is computed are not strictly perpendicular to the LEV trajectories. Thus, the term "LEV axial vorticity" is not strictly accurate in its present usage. Also, it should be mentioned that the computed sign of vorticity is the reverse of the general convention. This is strictly a consequence of sign conventions introduced by the mirrors in the stereo PIV experimental setup.

\subsection{1 $\quad 65^{\circ}$ wing vorticity plots}

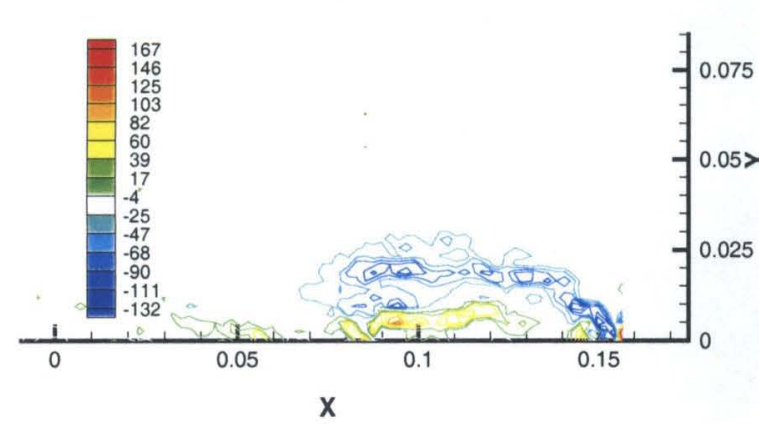

Figure 5.77. $65^{\circ}$ wing, $\alpha=5^{\circ}$

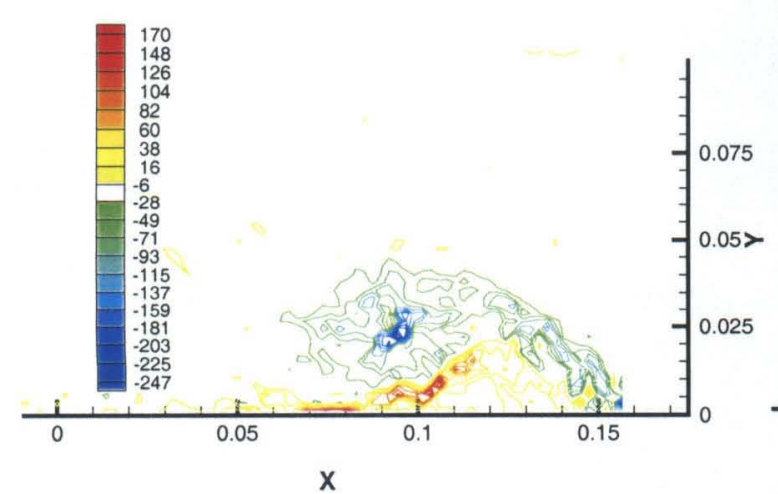

Figure 5.79. $65^{\circ}$ wing, $\alpha=10^{\circ}$

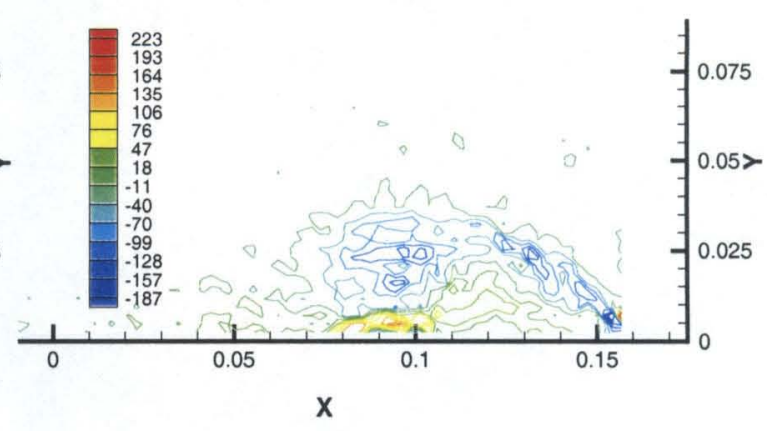

Figure 5.78. $65^{\circ}$ wing, $\alpha=7.5^{\circ}$

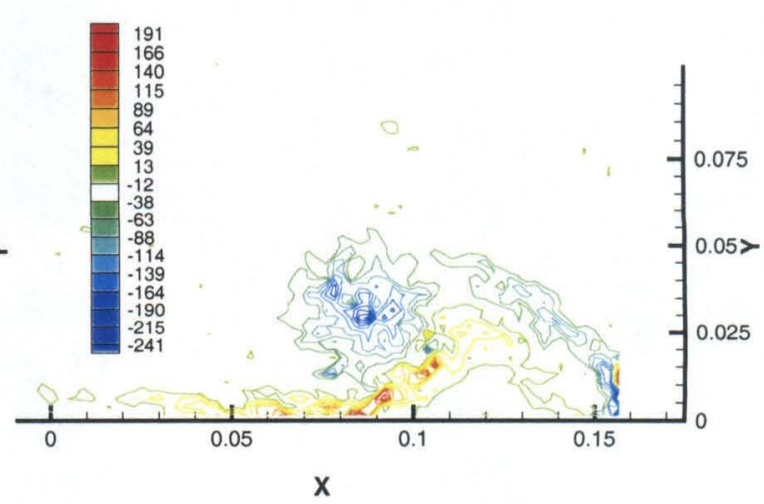

Figure 5.80. $65^{\circ}$ wing, $\alpha=12.5^{\circ}$ 


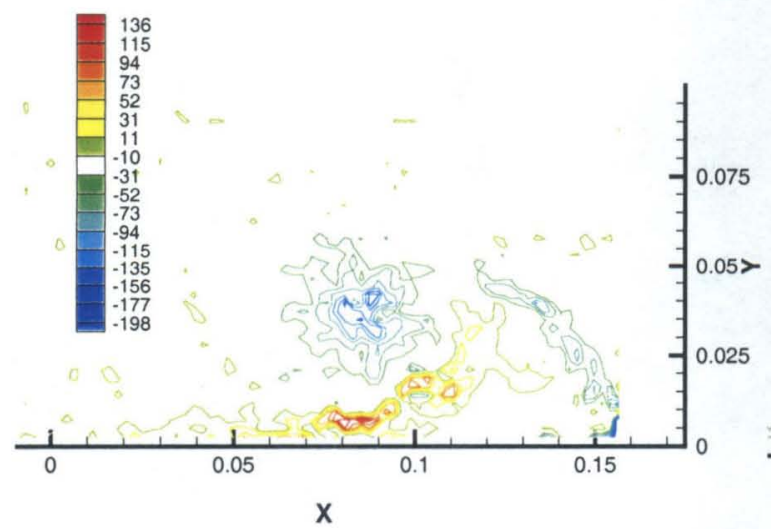

Figure 5.81. $65^{\circ}$ wing, $\alpha=15^{\circ}$

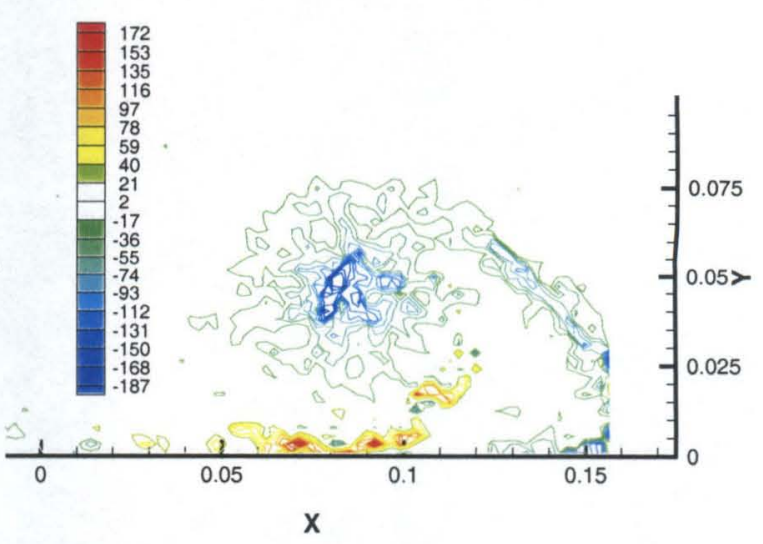

Figure 5.82. $65^{\circ}$ wing, $\alpha=20^{\circ}$

At $5^{\circ}$ and $7.5^{\circ}$ angles of attack, peak negative vorticity occurs in the LE shear layer. But by $10^{\circ} \mathrm{AOA}$, the peak of negative vorticity becomes associated with the LEV core. This is to be expected, as the LEV strengthens with increasing angle of attack.

\subsection{2 $50^{\circ}$ wing vorticity plots}

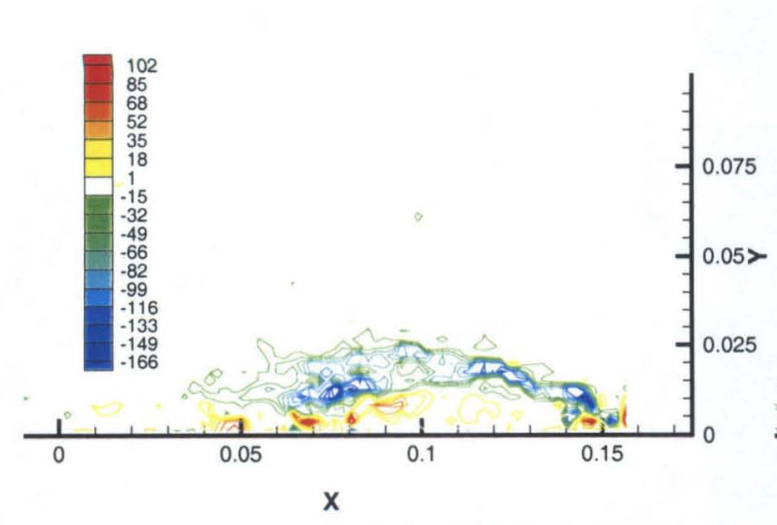

Figure 5.83. 50 $^{\circ}$ wing, $\alpha=5^{\circ}$

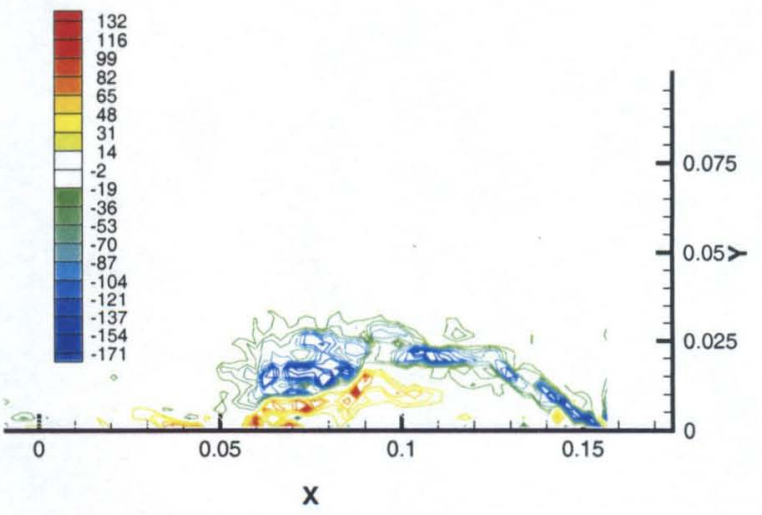

Figure 5.84. $50^{\circ}$ wing, $\alpha=7.5^{\circ}$ 


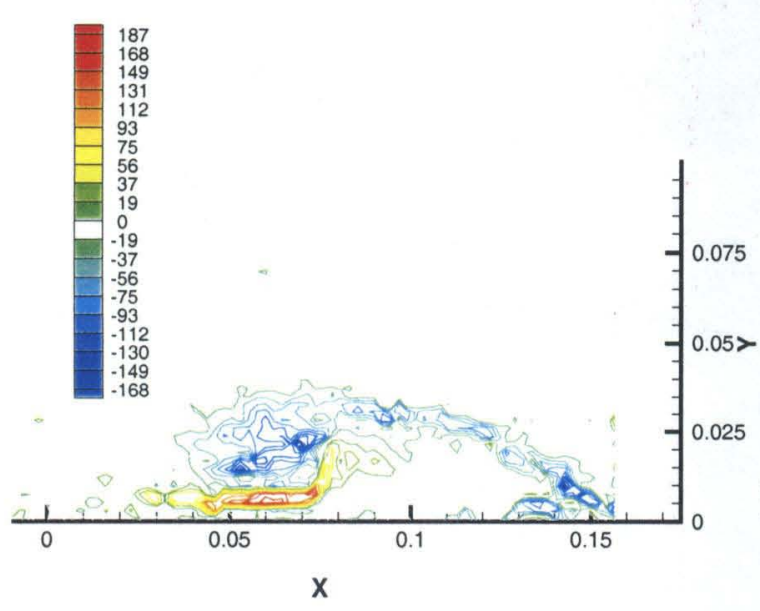

Figure 5.85. 50 ${ }^{\circ}$ wing, $\alpha=10^{\circ}$

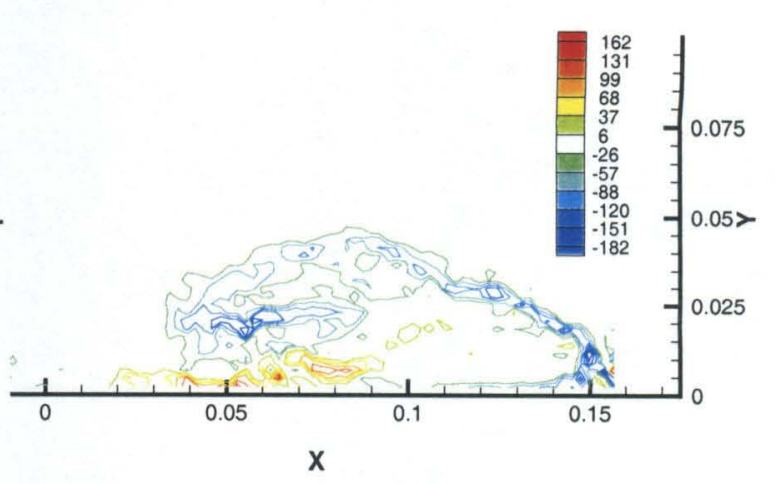

Figure 5.86. $50^{\circ}$ wing, $\alpha=12.5^{\circ}$

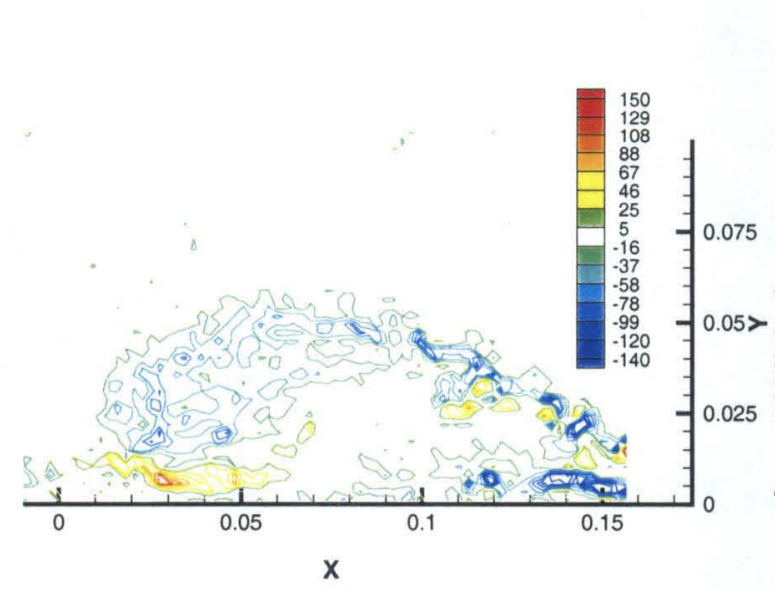

Figure 5.87. $50^{\circ}$ wing, $\alpha=15^{\circ}$

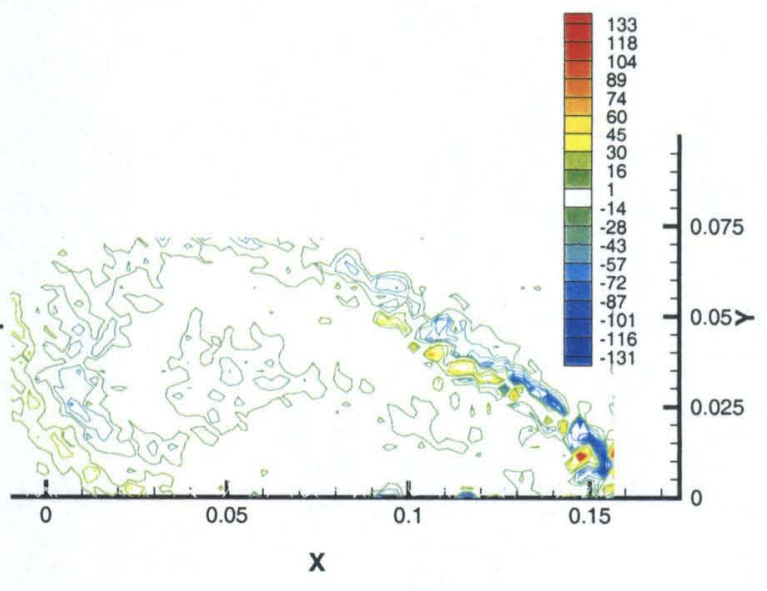

Figure 5.88. $50^{\circ}$ wing, $\alpha=20^{\circ}$

All of the vorticity plots, with the exception of the $15^{\circ}$ and especially the $20^{\circ}$ angle of attack $50^{\circ}$ wing plots, show at least a local axial vorticity peak in the general vicinity of the primary LEV core. A "slab" of vorticity of the opposite sense near the leeward boundary layer is also present, starting approximately at the primary LEV attachment line, and proceeding outboard. Again with the exception of the $20^{\circ}$ angle of attack, the sub-structures of local vorticity peaks in each family are mutually of the same sign, ruling out the presence of counter-rotating vortex pairs. The region between the boundary layer vorticity "slab" and the leading edge shear layer is largely devoid of vorticity, further supporting the assertion that this flow is essentially stagnant. 
From the $50^{\circ}$ wing plots, it can be seen that in going from $10^{\circ}$ to $12.5^{\circ}$ to $15^{\circ}$ angle of attack, the strong axial vorticity in the primary core is dissipated. By $20^{\circ}$, the "interior" of the rolled-up shear layer has no discernable vorticity peaks. Interestingly, the leading edge shear layer displays vorticity sub-structures of both signs. This has implications of the balance of vorticity production and convection over the entire flowfield of the wing. If the LE shear layer contains contour-rotating structures, there is no longer the need to sustain a stable LEV as a downstream sink of vorticity, as would have been the case were the vorticity in the LE shear layer all of one sign.

\subsubsection{3-components of vorticity for selected cases}

These data were taken for the $50^{\circ}$ wing for $\alpha=10^{\circ}$ and $15^{\circ}$, and for the $65^{\circ}$ wing for $\alpha=15^{\circ}$. For these cases, the utility of stereo PIV can be extended to obtain three dimensional vorticity, albeit in the averaged sense, as discussed above. Vorticity is shown as a vector field. First, the streamwise view is given. The color bar corresponds to the total magnitude, whence there are no negative values. Next, the top view is shown, looking over the wing planform. Now the color bar corresponds to just the out-of-plane vorticity component.

\subsubsection{1 $65^{\circ}$ wing $\alpha=15^{\circ}$}




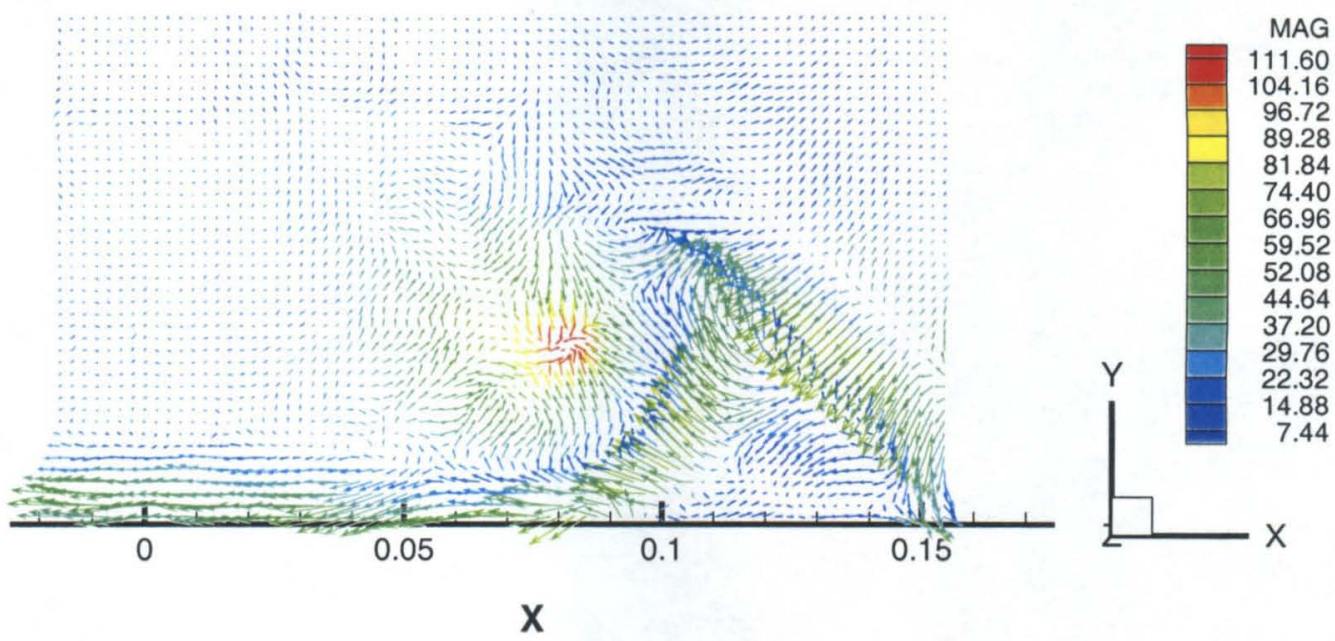

Figure 5.89. $65^{\circ}$ wing, $\alpha=15^{\circ}$, mean 3-component vorticity field
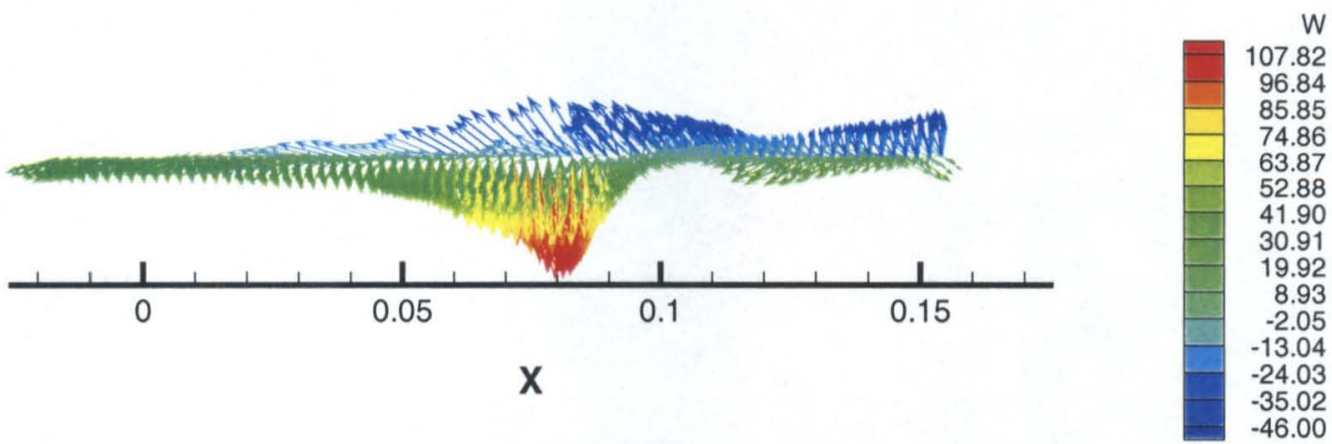

Figure 5.90. $65^{\circ}$ wing, $\alpha=15^{\circ}$, mean 3-component vorticity field, top view

Here we see strong out-of-plane vorticity in the LEV core, as expected for a slender delta wing. Vorticity in the shear layer is not as strong as for the $50^{\circ}$ case. Vorticity at the wing leeward surface inboard of the primary attachment line is indicative of that produced by a boundary layer velocity profile - and thus, of attached boundary layer flow.

It should be noted that the peak value of out-of-plane vorticity component is smaller than that of the contour plots in Section 5.5.1 and 5.5.2. The reason is that the numerical method used to compute out-of-plane vorticity from planar data, used in 
Sections 5.5.1 and 5.5.2 (described more fully in ${ }^{63}$ ), is less dissipative than the differencing method described in Section 2.11.

\subsubsection{2 $50^{\circ}$ wing $\alpha=10^{\circ}$}

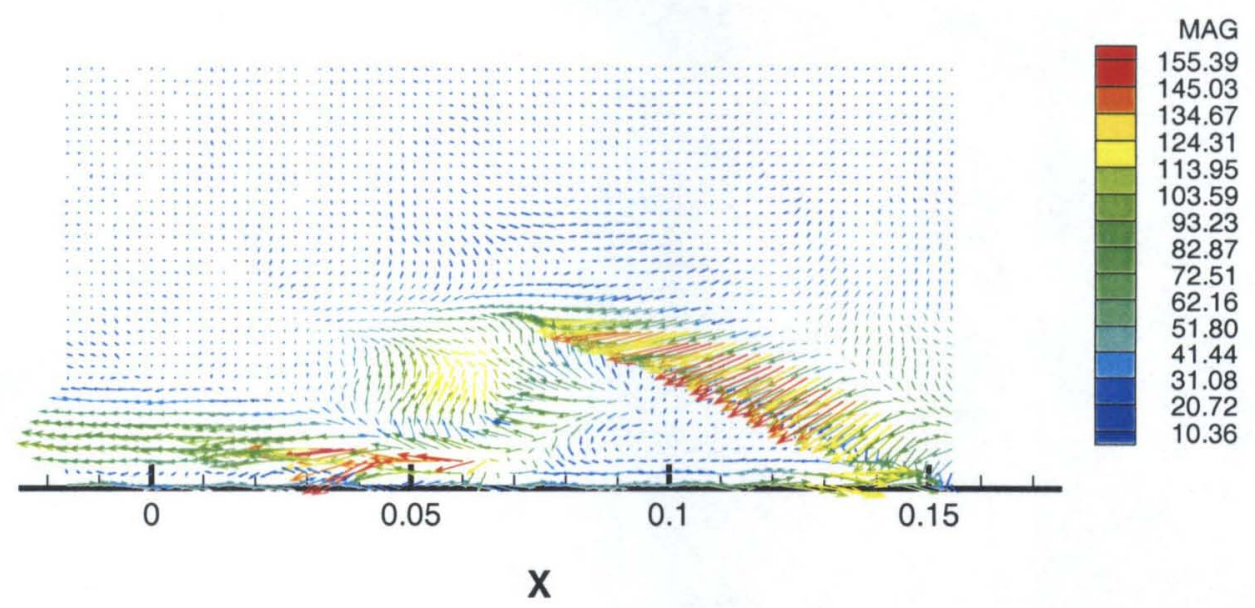

Figure 5.91. $50^{\circ}$ wing, $\alpha=10^{\circ}$, mean 3-component vorticity field

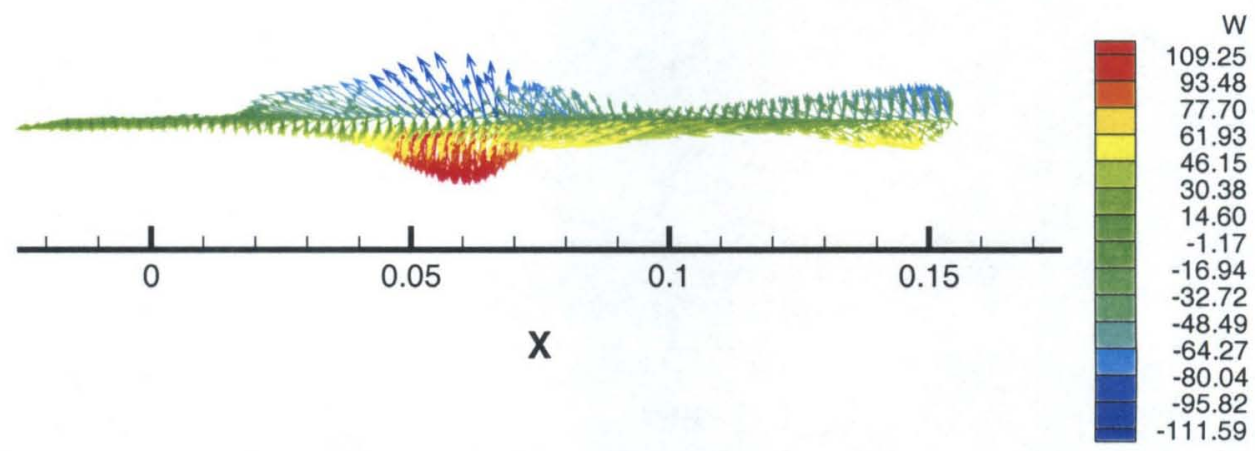

Figure 5.92. $50^{\circ}$ wing, $\alpha=10^{\circ}$, mean 3-component vorticity field, top view

There is definite evidence of a coherent leading edge vortex, in some contrast to the situation in 5.5.3.3 below. A boundary layer profile on spanwise near-surface flow underneath the LEV is evidently responsible for what is shown as negative streamwise vorticity in Figure 5.92. Vorticity associated with the LEV dominates that of the shear layer, at least for the out-of-plane component. 


\subsubsection{50 ${ }^{\circ}$ wing $\alpha=15^{\circ}$}

The $50^{\circ}$ wing $\alpha=15^{\circ}$ case exhibits appreciably less vorticity in the region bounded by the rolling-up shear layer.

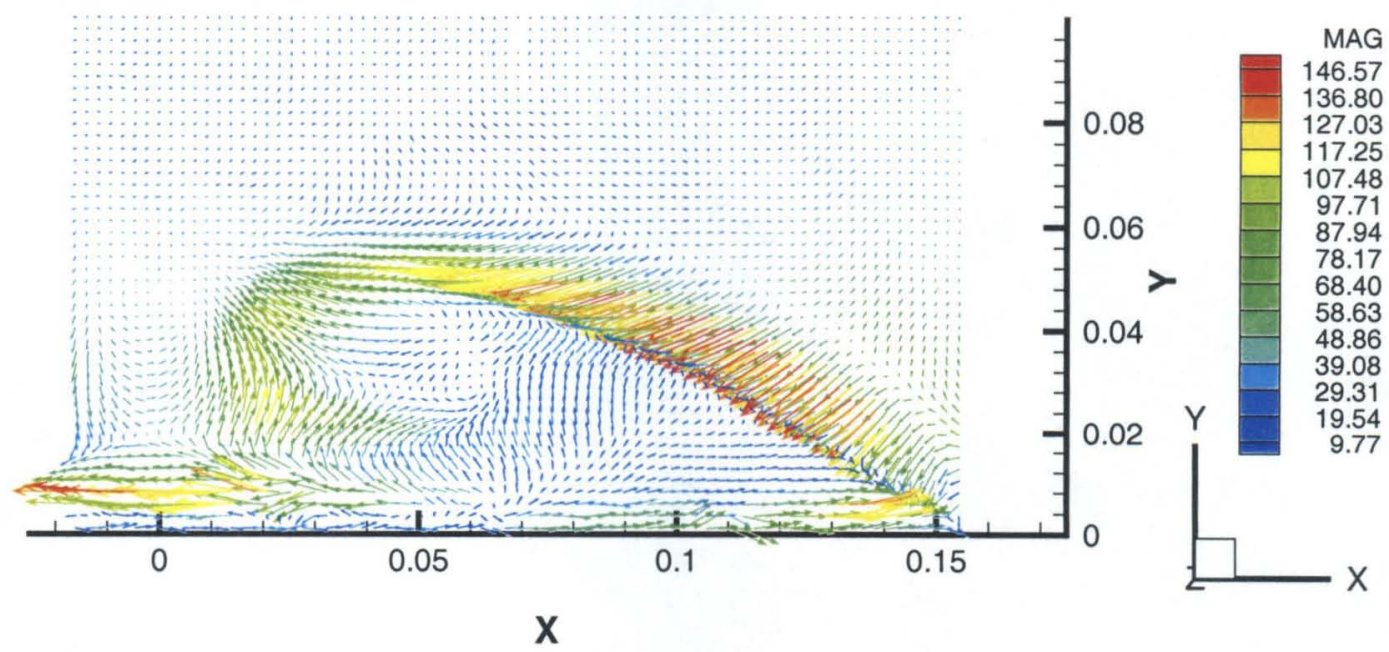

Figure 5.93. $50^{\circ}$ wing, $\alpha=15^{\circ}$, mean 3-component vorticity field

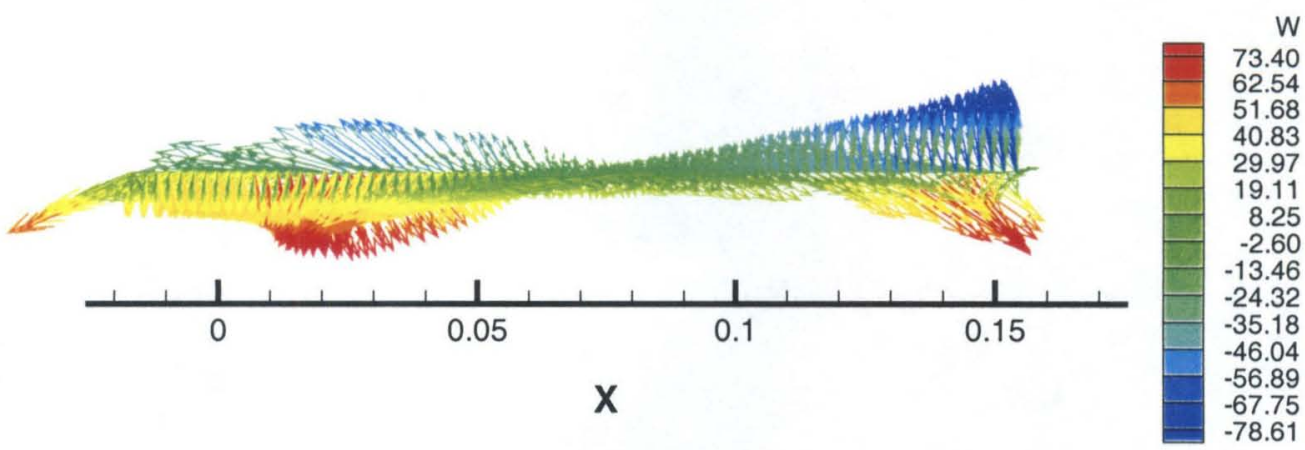

Figure 5.94. $50^{\circ}$ wing, $\alpha=15^{\circ}$, mean 3-component vorticity field, top view

The top view of the vorticity field, colored by contours of the out-of-plane component (Figure 5.94) shows that there is still a vortical structure identifiable as the primary LEV. But the vorticity in the shear layer, especially the region nearest the leading edge, is certainly dominant.

\subsection{Images near the apex}


We now consider an interrogation region closer to the wing apex. In the following, data for the $50^{\circ}$ wing were taken at $12.7 \mathrm{~mm}$ downstream of the apex, corresponding to $\mathrm{z} / \mathrm{c}=0.12$. For the $65^{\circ}$ wing, data at $19.1 \mathrm{~mm}$, corresponding to $\mathrm{z} / \mathrm{c}=$ 0.10 , are presented. Because the nondimensional scaling for the two wings differs slightly in this case, quantitative comparison of LEV size should be treated with some care. However, the qualitative distinctions are easily discernable. A selection of $50^{\circ}$ wing images are given in Section $5.6 .1 ; 65^{\circ}$ wing images are presented in the subsequent section.

\subsection{1 $50^{\circ}$ wing near-apex images}

As in Section 5.3, data are presented for the $5^{\circ}, 7.5^{\circ}, 10^{\circ}, 12.5^{\circ}, 15^{\circ}$, and $20^{\circ}$ angle of attack cases. Streamwise and top views of the instantaneous velocity vector field are given. These are followed by streamwise views of the mean and root-meansquare velocity fields.

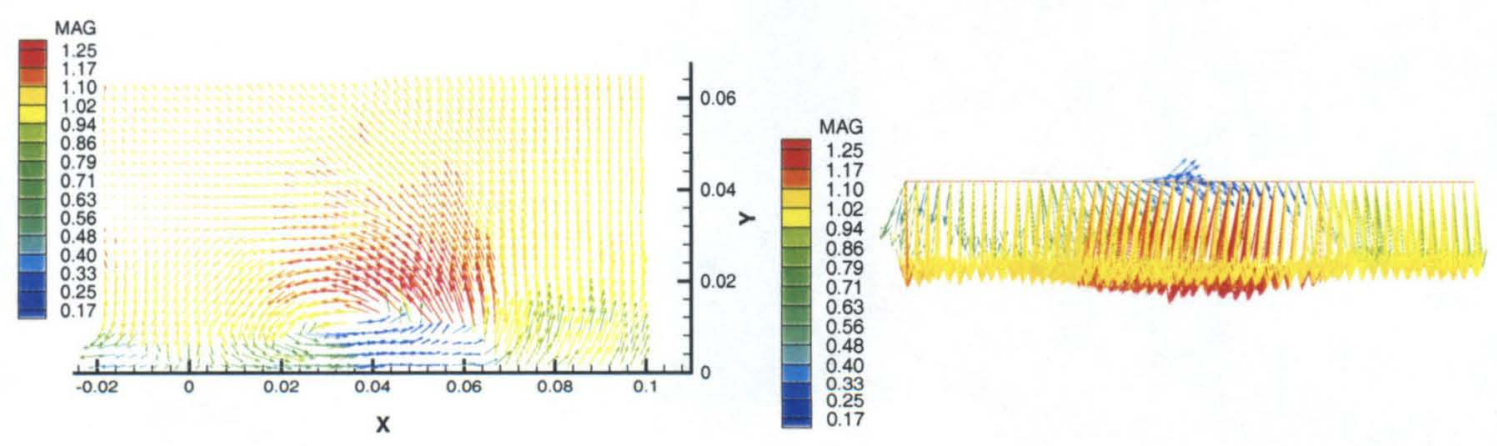

Figure 5.95. $50^{\circ}$ wing, $\alpha=5^{\circ}$, instantaneous velocity field 


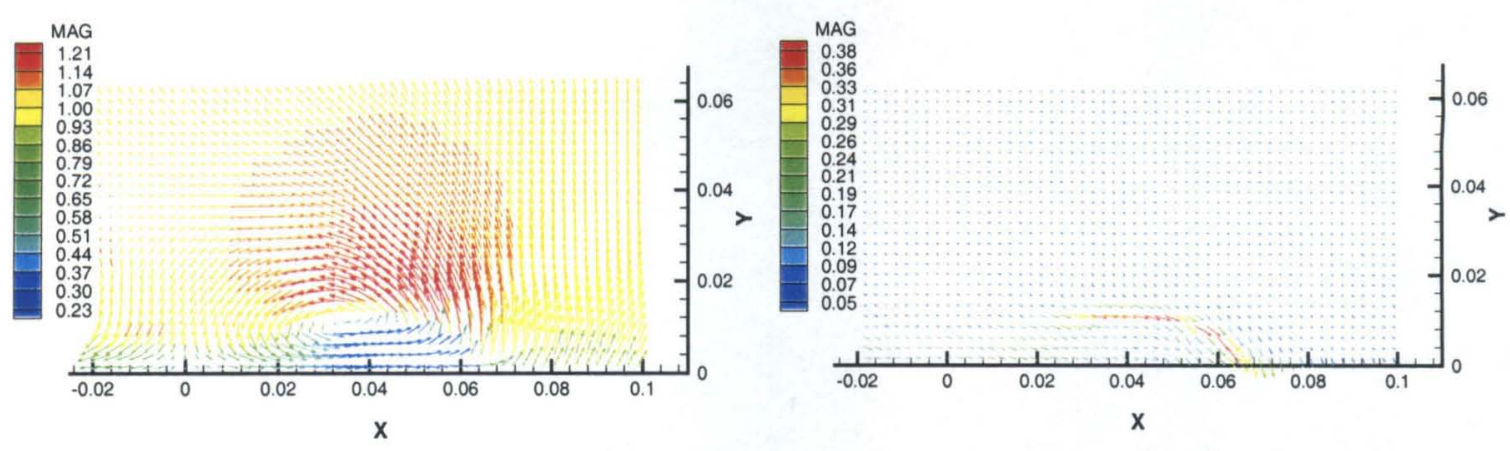

Figure 5.96. $50^{\circ}$ wing, $\alpha=5^{\circ}$, mean and $\mathrm{rms}$ velocity fields

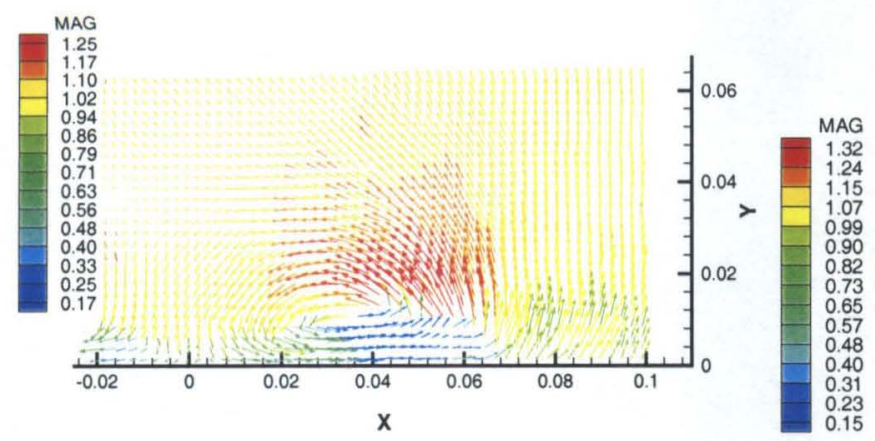

Figure 5.97. $50^{\circ}$ wing, $\alpha=7.5^{\circ}$, instantaneous velocity field

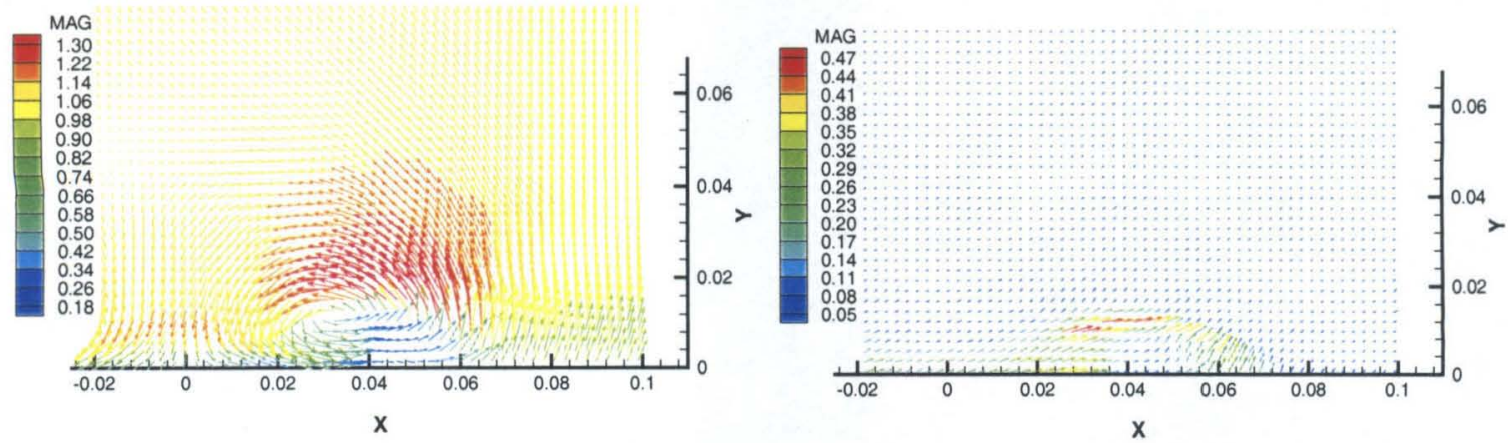

Figure 5.98. $50^{\circ}$ wing, $\alpha=7.5^{\circ}$, mean and rms velocity fields

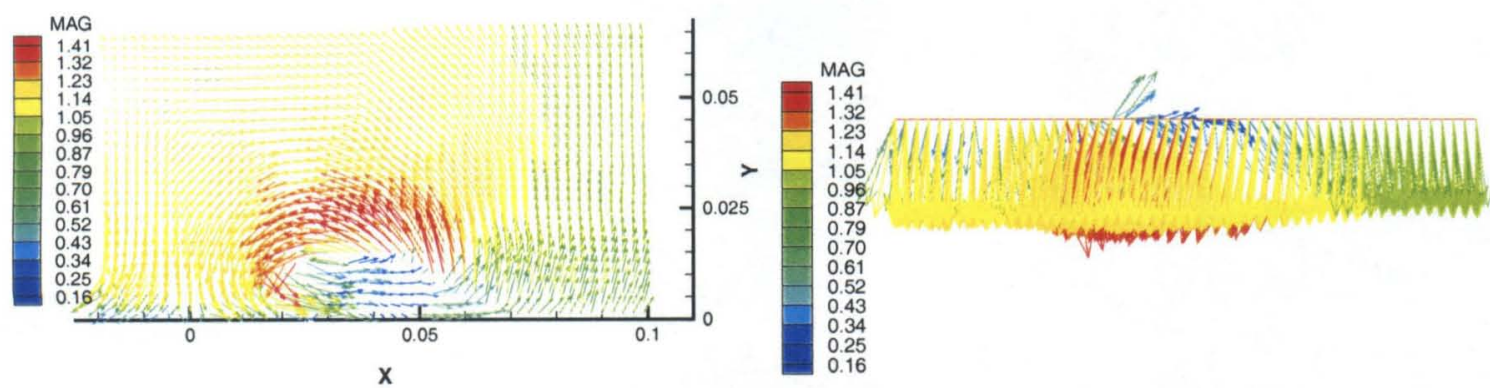

Figure 5.99. $50^{\circ}$ wing, $\alpha=10^{\circ}$, instantaneous velocity field 


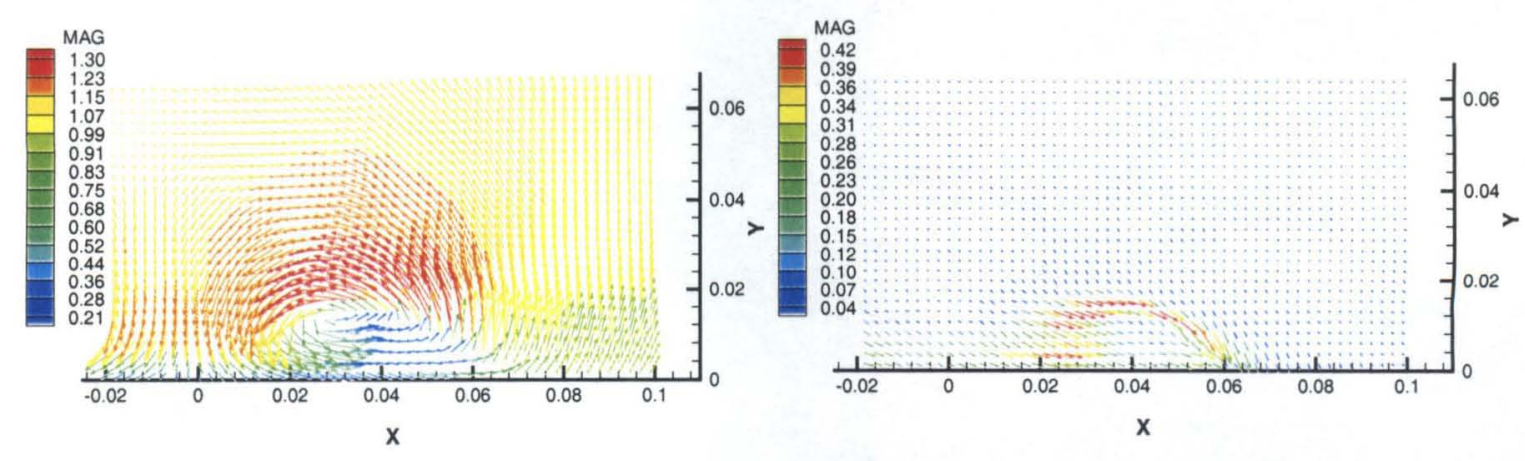

Figure 5.100. $50^{\circ}$ wing, $\alpha=10^{\circ}$, mean and $\mathrm{rms}$ velocity fields

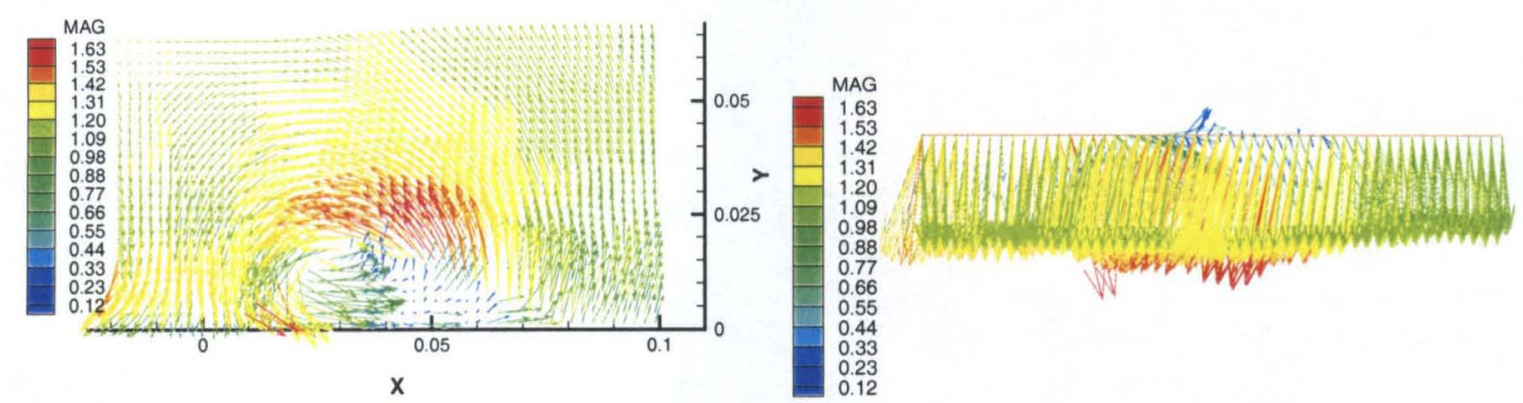

Figure 5.101. $50^{\circ}$ wing, $\alpha=12.5^{\circ}$, instantaneous velocity field

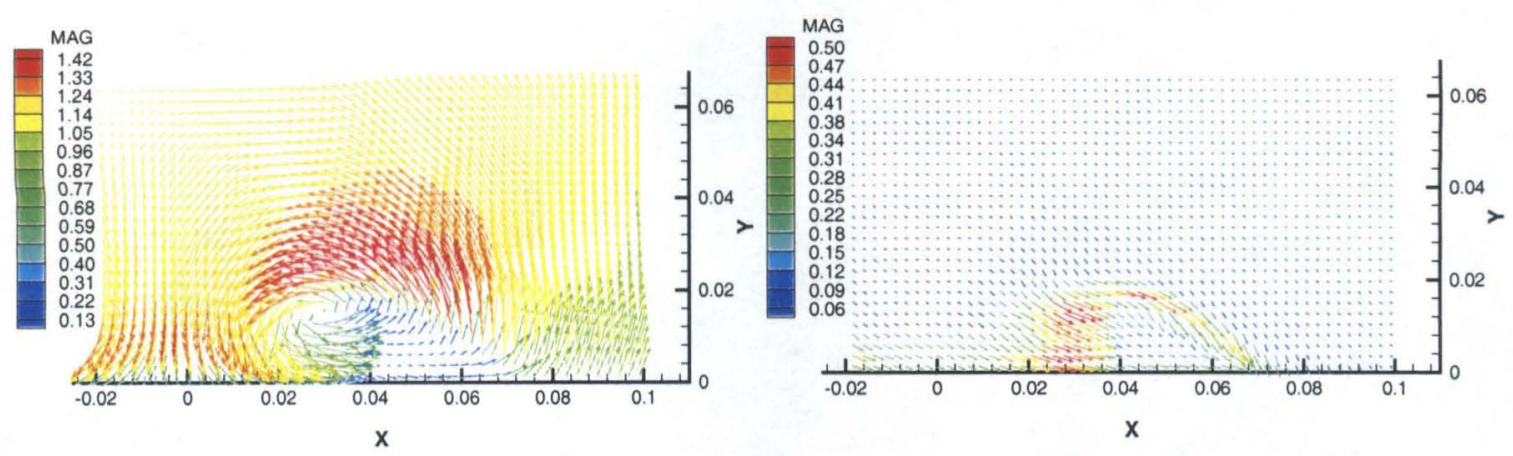

Figure 5.102. $50^{\circ}$ wing, $\alpha=12.5^{\circ}$, mean and rms velocity fields

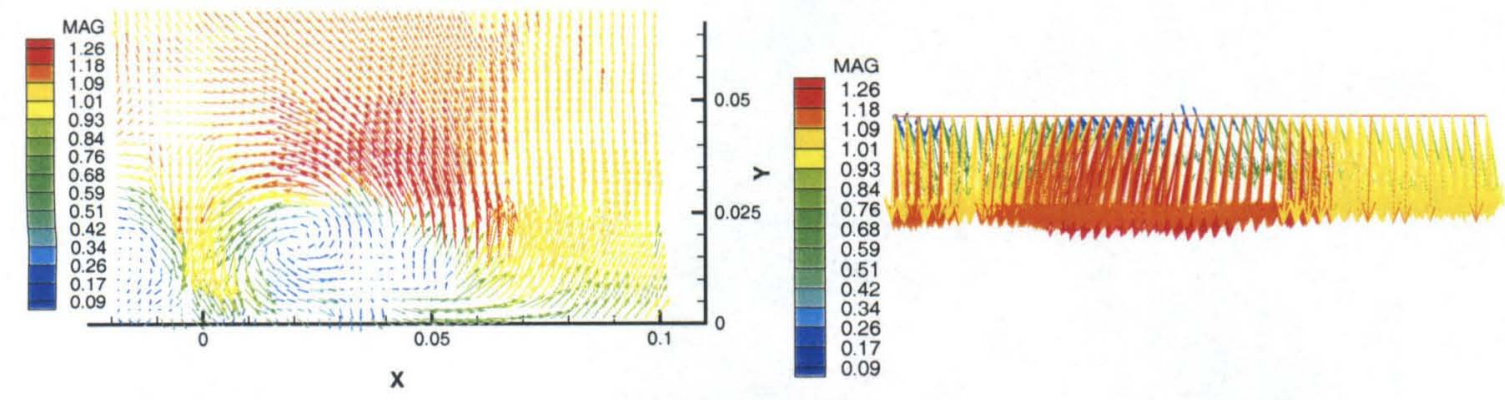

Figure 5.103. $50^{\circ}$ wing, $\alpha=15^{\circ}$, instantaneous velocity field 


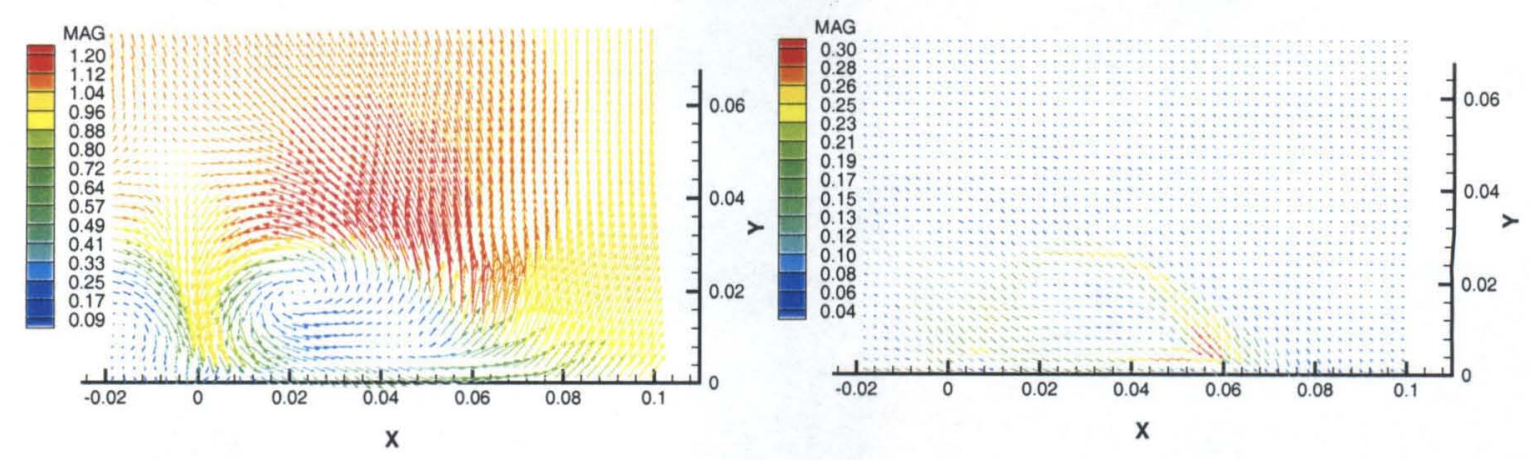

Figure 5.104. $50^{\circ}$ wing, $\alpha=15^{\circ}$, mean and rms velocity fields

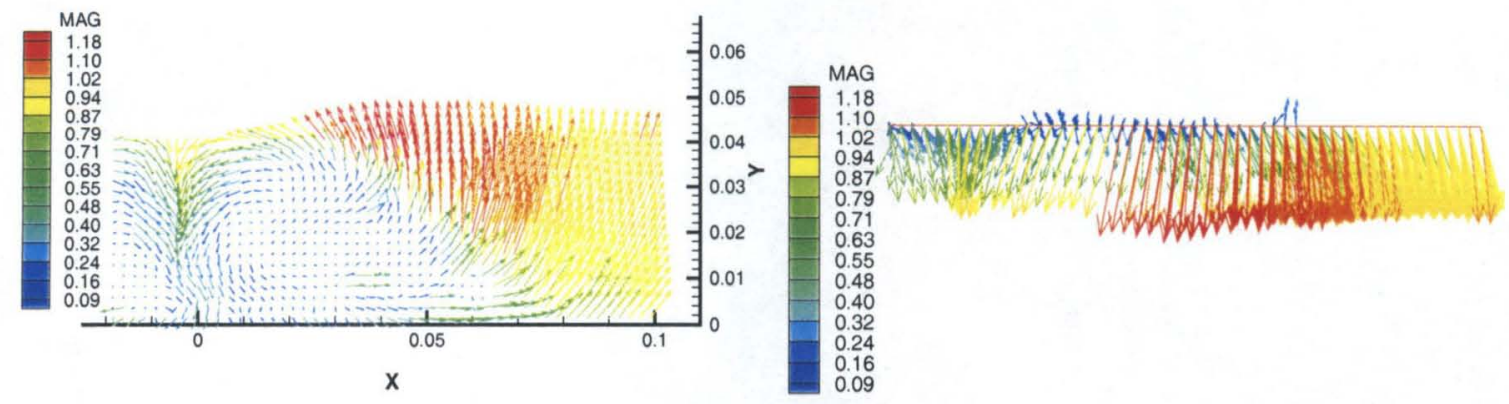

Figure 5.105. $50^{\circ}$ wing, $\alpha=20^{\circ}$, instantaneous velocity field

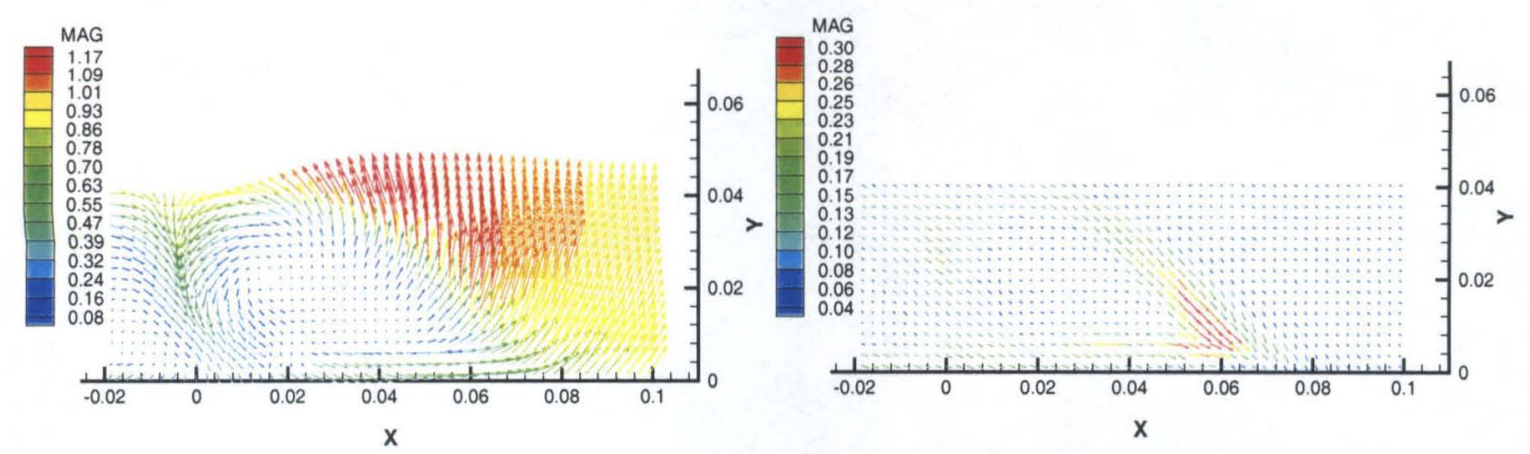

Figure 5.106. $50^{\circ}$ wing, $\alpha=20^{\circ}$, mean and rms velocity fields

\subsection{2 $65^{\circ}$ wing near-apex images}

Velocity vector plots for $7.5^{\circ}, 12.5^{\circ}$, and $20^{\circ}$ angle of attack are shown below. The data are presented more sparsely than in the section above, because changes with increasing angle of attack are less severe. 


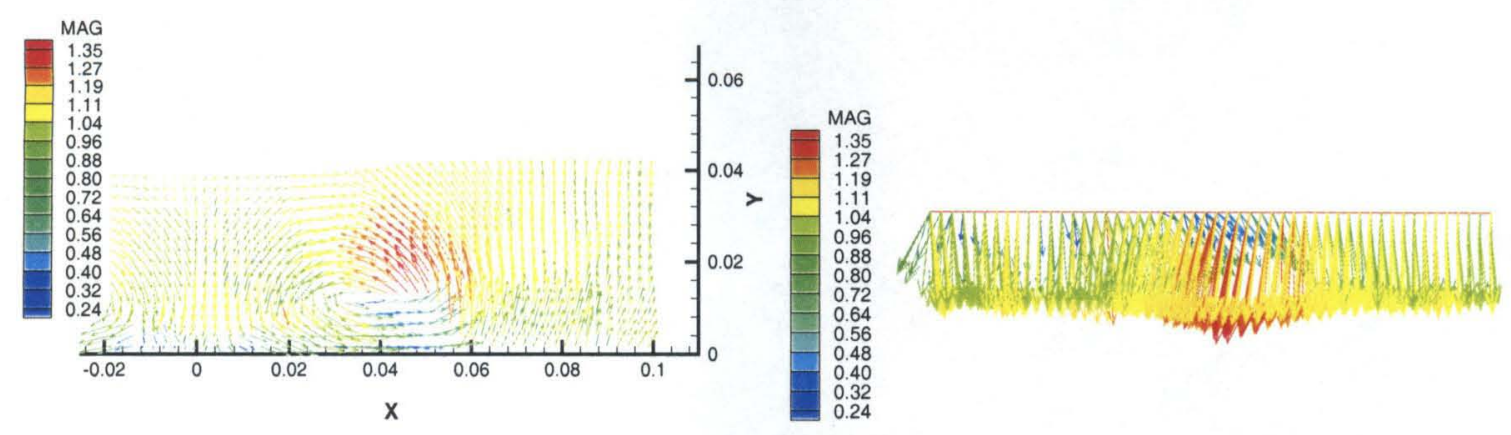

Figure 5.107. $65^{\circ}$ wing, $\alpha=7.5^{\circ}$, instantaneous velocity field

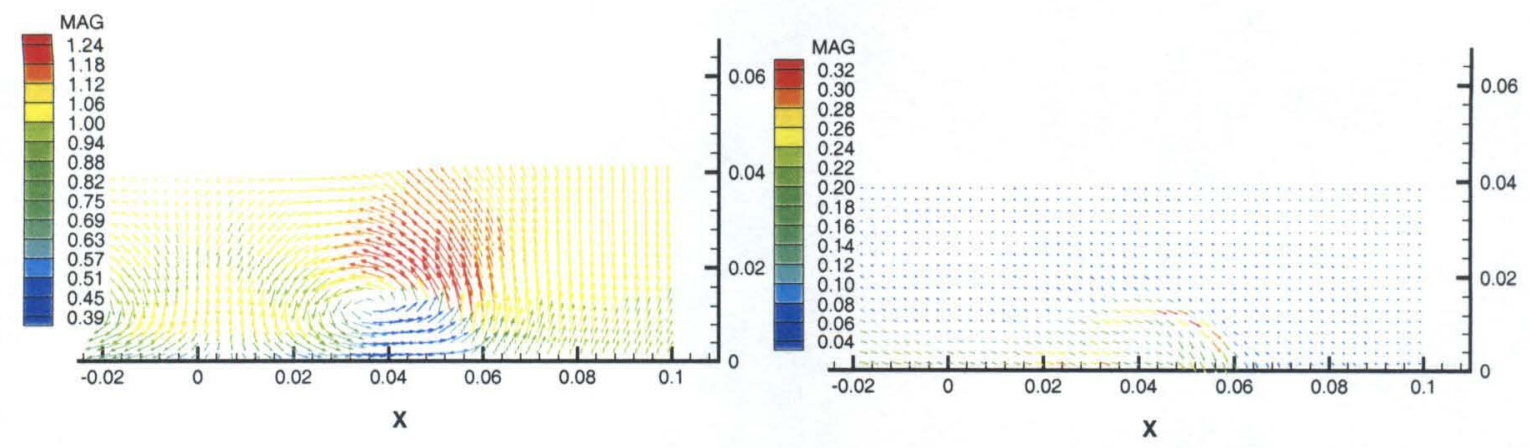

Figure 5.108. $65^{\circ}$ wing, $\alpha=7.5^{\circ}$, mean and rms velocity fields

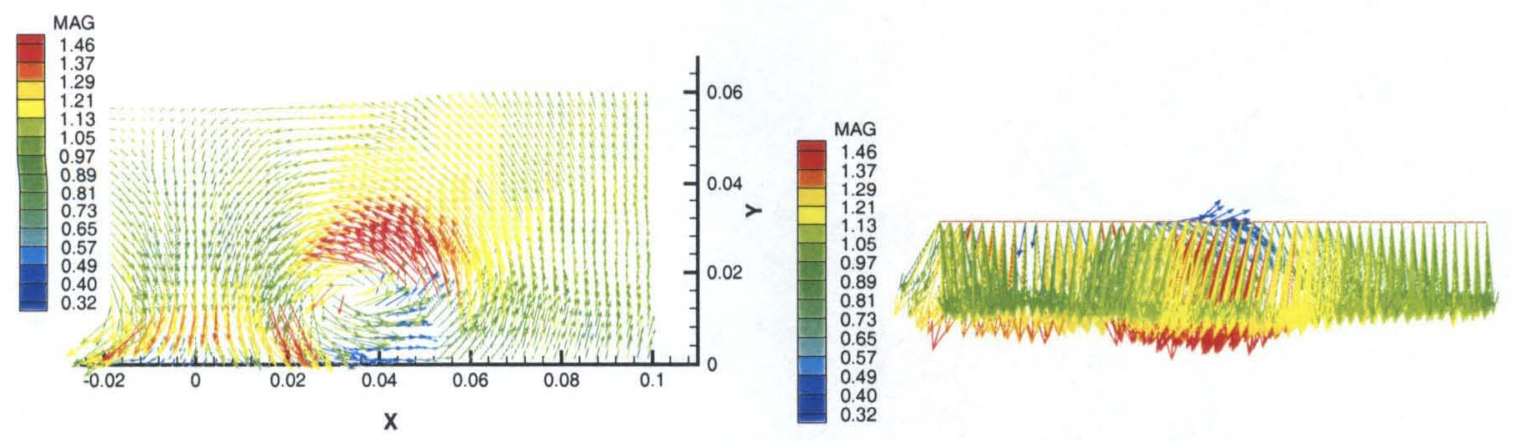

Figure 5.109. $65^{\circ}$ wing, $\alpha=12.5^{\circ}$, instantaneous velocity field

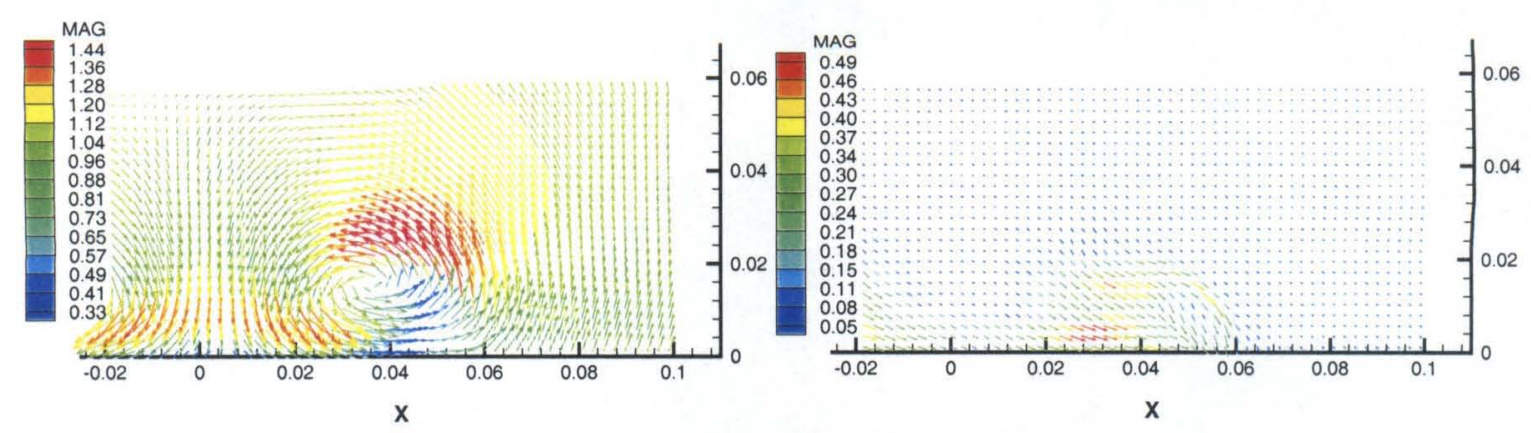

Figure 5.110. $65^{\circ}$ wing, $\alpha=12.5^{\circ}$, mean and rms velocity fields 


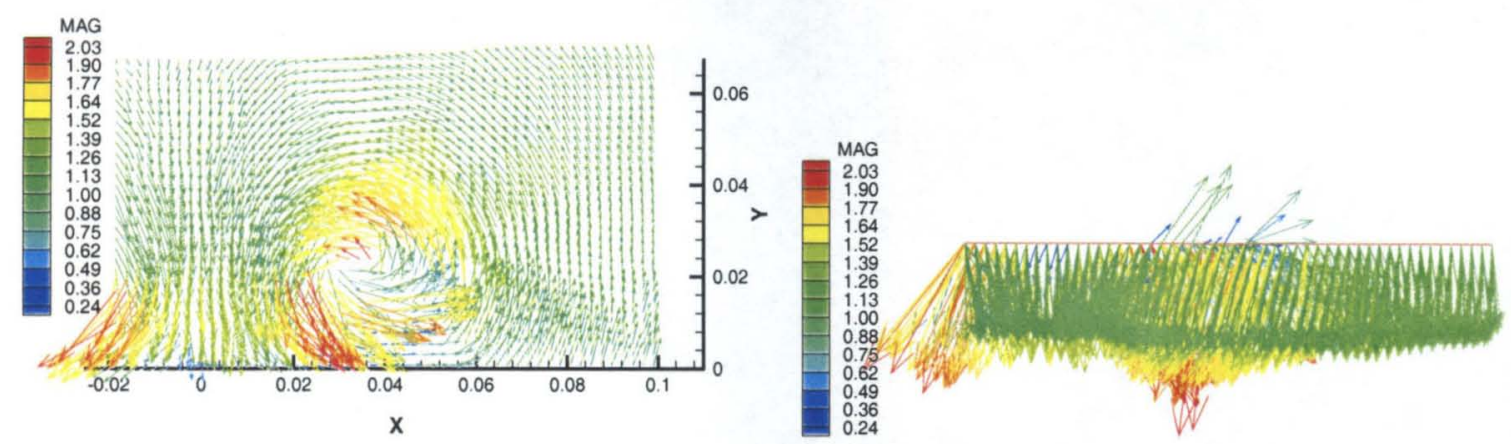

Figure 5.111. $65^{\circ}$ wing, $\alpha=20^{\circ}$, instantaneous velocity field

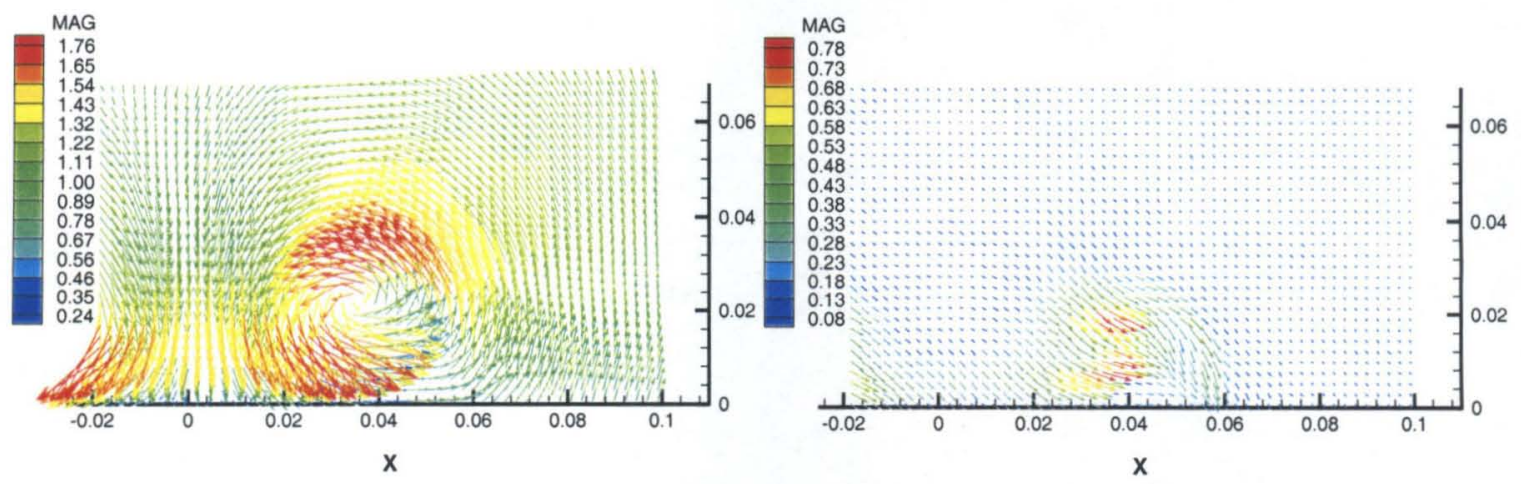

Figure 5.112. $65^{\circ}$ wing, $\alpha=20^{\circ}$, mean and $\mathrm{rms}$ velocity fields

\subsubsection{A summary of near-apex flow features}

For both wings, unsteadiness in the velocity field is greater near the apex than at $z / c=0.3$. This is seen in the rms velocity plots as a greater spatial extent of highfluctuation regions. On a speculative basis, this can be attributed to the fact that closer to the apex, the primary and secondary LEVs are closer together, and their mutual interaction, along with the interaction of the two with the leeward surface boundary layer, results in greater temporal variations. 


\subsection{Vortex breakdown and conical flow}

\subsubsection{Some general comments}

As noted in previous chapters, there are numerous criteria for identifying the presence of vortex breakdown of the LEVs. All of these criteria share the common theme that the concentrated vorticity of the unbroken LEV core has been suddenly and substantially attenuated in magnitude, while of course, conservation of the total circulation is enforced. Here we are concerned with evidence from the PIV images that 1) LEV core vorticity has undergone a qualitative change in this sense, and 2) the LEV core axial velocity profile has transitioned from a "jet-like" to a "wake-like" profile.

On this basis, the downstream location of VB could in principle be estimated from the SPIV data, for those cases where VB was not downstream of the domain of the PIV data set. For the $65^{\circ}$ wing, VB was well downstream of the aft-most SPIV interrogation plane, located at $\mathrm{z} / \mathrm{c}=0.362$ over the range of angles of attack under consideration. For the $50^{\circ}$ wing, breakdown location was also downstream of the aft-most SPIV station for $10^{\circ}$ angle of attack and below. However, for the $20^{\circ}$ angle of attack, breakdown was seen even on the most forward station, at $\mathrm{z} / \mathrm{c}=0.12$ (see Section 5.6.1).

The intermediate angles of attack, $12.5^{\circ}$ and $15^{\circ}$, are the most interesting. It is for these conditions, and only these, that discernable VB occurred within the domain covered by the SPIV tests. As discussed in Chapter 5, strong unsteadiness in VB behavior was seen for these angles from the flow visualization. These strong Z-direction undulations invite a more systematic comparison of SPIV data over crossflow planes at multiple $z / c$ locations. These are considered below. 


\subsubsection{The $50^{\circ}$ wing at intermediate angles of attack}

In the following, streamwise views of the mean velocity field are given for the $50^{\circ}$ wing at $12.5^{\circ}$ and $15^{\circ}$ angles of attack, filling in the interrogation plane locations between $\mathrm{z} / \mathrm{c}=0.12$ and $\mathrm{z} / \mathrm{c}=0.3$; that is, $\mathrm{z} / \mathrm{c}=0.178$ and 0.237 . This information will help to further elucidate the location of VB for these angles of attack.

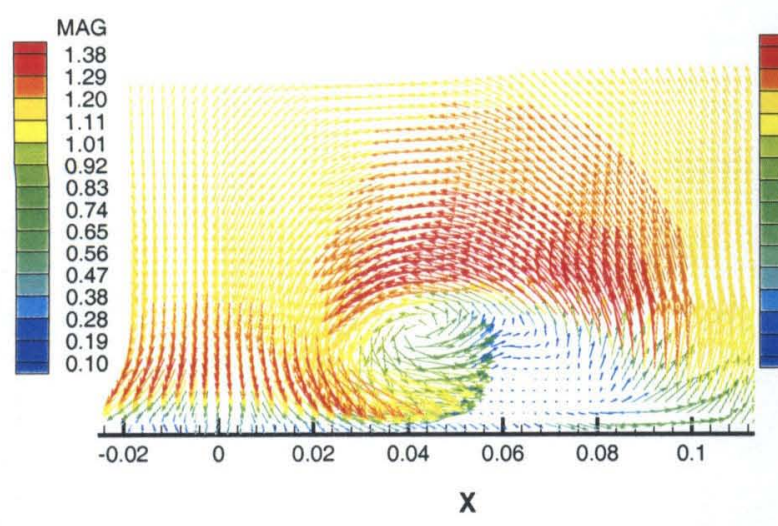

Figure 5.113. $\alpha=12.5^{\circ}, \mathrm{z} / \mathrm{c}=\mathbf{0 . 1 7 8}$

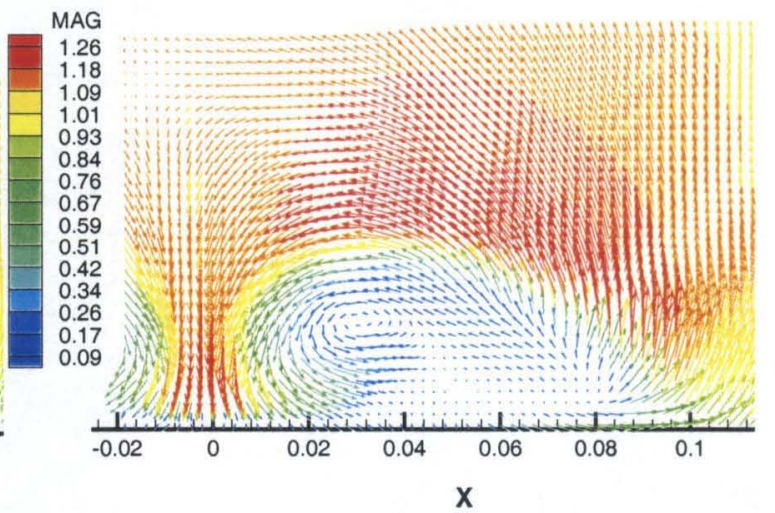

Figure 5.114. $\alpha=15^{\circ}, \mathrm{z} / \mathrm{c}=\mathbf{0 . 1 7 8}$

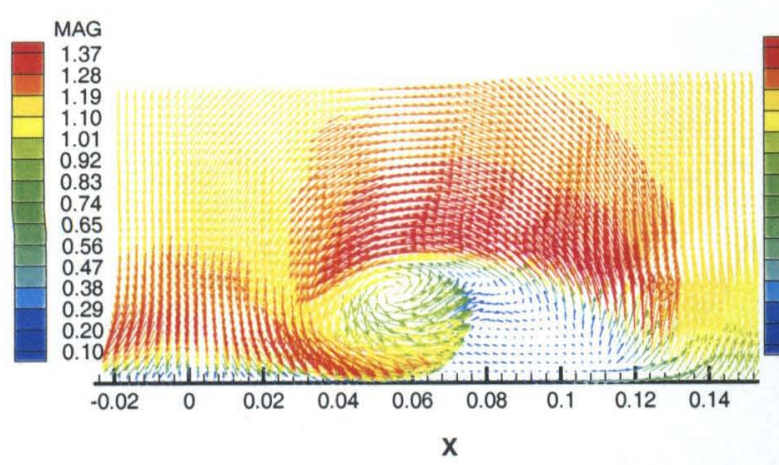

Figure 5.115. $\alpha=12.5^{\circ}, \mathrm{z} / \mathrm{c}=\mathbf{0 . 2 3 7}$

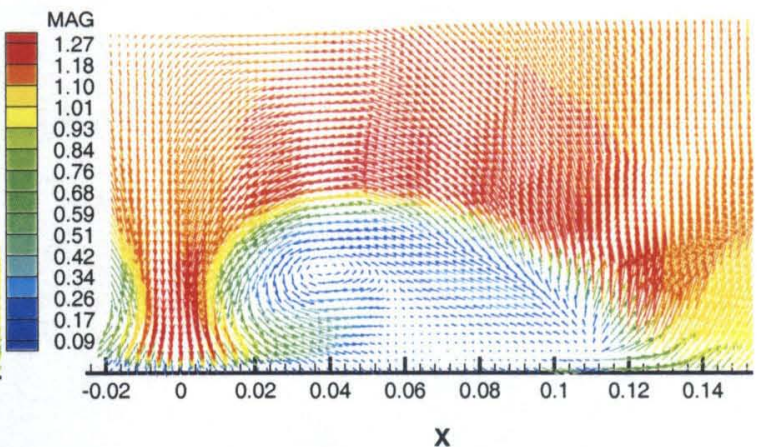

Figure 5.116. $\alpha=15^{\circ}, \mathrm{z} / \mathrm{c}=\mathbf{0 . 2 3 7}$

It is useful to consider axial velocity profiles of the $\mathrm{z} / \mathrm{c}=0.118,0.178,0.237$, and 0.296 stations, for the $50^{\circ}$ wing at $12.5^{\circ}$ and $15^{\circ}$ angles of attack. These are shown in

Figure 5.117. The abscissas for each $\mathrm{z} / \mathrm{c}$ station were rescaled by the local semi-span, so that the LE location for all curves is at $x=0.5$. The methodology is discussed in further 
detail in Section 6.2, where similar curves are presented for both the $50^{\circ}$ and $65^{\circ}$ wings for a range of angles of attack.

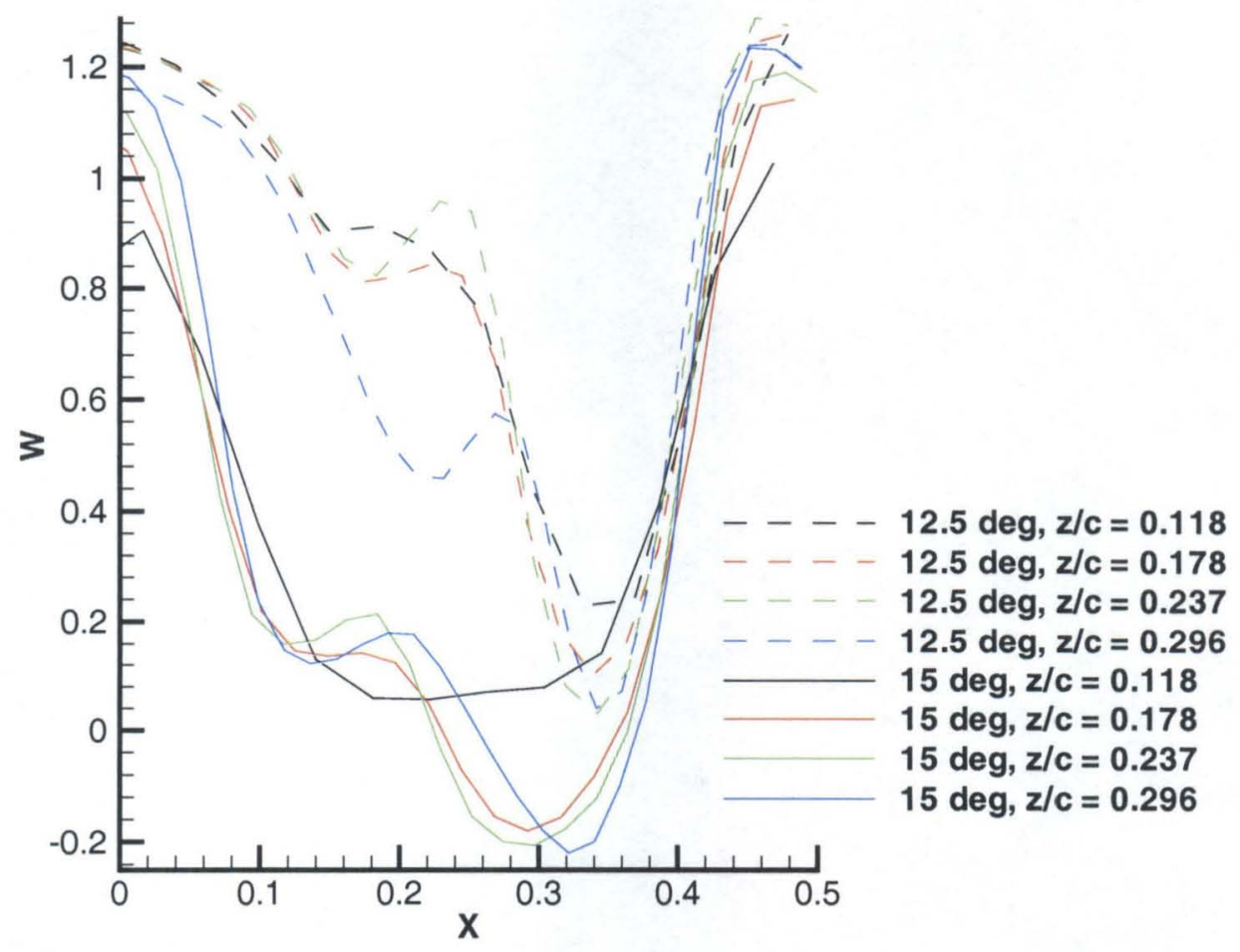

Figure 5.117. Mean axial velocity profiles for $\alpha=12.5^{\circ}$ and $15^{\circ}$

With the rescaling described above, data for both the $12.5^{\circ}$ and $15^{\circ} \mathrm{AOA}$ become nearly invariant with $\mathrm{z} / \mathrm{c}$ station. This is strong indication of a conical velocity field, in the language of Section 1.2.2. With the exceptions of the $\mathrm{z} / \mathrm{c}=0.11815^{\circ}$ curve, and the $\mathrm{z} / \mathrm{c}=0.29612 .5^{\circ}$ curve, data in both AOA group almost overlap. In all the curves, the peak in axial velocity occurring near $\mathrm{x}=0.45$ corresponds to the region just outboard of the LE shear layer, where the velocity magnitude is slightly larger than free stream.

It is evident that for the $12.5^{\circ}$ angle of attack case, axial flow in the LEV core is not appreciably retarded at any of the $\mathrm{z} / \mathrm{c}$ stations. At the most aft station, axial flow is 
slightly smaller, evidently attributable to a stronger influence of breakdown. Outboard of the LEV, flow is strongly retarded, with a velocity magnitude of about $0.2 U_{\infty}$.

For the $15^{\circ} \mathrm{AOA}$ case, the velocity profiles are rather different. Axial flow everywhere inside the region bounded by the rolled-up LE shear layer is markedly slower than the free stream speed, with the region outboard of the LEV remnant having reversed flow. The most upstream station lacks the outboard region of reversed flow, but it also lacks a discernable local velocity peak attributable to a LEV.

In terms of the mean axial velocity profiles, it is not unreasonable to conclude that at $12.5^{\circ}$ angle of attack, vortex breakdown occurs downstream of the domain of interrogation, but reaches the apex at $15^{\circ}$ angle of attack. This is consistent with the flow visualizations, where the average VB location at $12.5^{\circ}$ was at $\mathrm{z} / \mathrm{c} \sim 0.5$, whereas at $15^{\circ}$, on VB location in an averaged sense could be elucidated.

\subsection{Summary}

Stereo PIV images were collected in crossflow planes loosely fitted to the rightside LEV trajectories of the $50^{\circ}$ and $65^{\circ}$ wings. While this arrangement does maximize the extent of the flow region of interest that can be captured, it is limited in observing regions further downstream, where flow visualization points out interesting "whorling" behavior in the secondary vortices. The present implementation of stereo PIV would benefit from a generalization, allowing for more rapid realignment and a program of more extensively imaging the flowfield.

Both wings are seen to exhibit coherent LEVs at $10^{\circ}$ angle of attack and below. Some evolutionary changes are observed for the $65^{\circ}$ wing at $12.5^{\circ}$ angle of attack, 
whereas the changes for the $50^{\circ}$ wing are substantial. In going to $20^{\circ}$ angle of attack, the $65^{\circ}$ wing flowfield undergoes changes limited to the strengthening of the primary LEV. The $50^{\circ}$ wing, however, undergoes vortex breakdown in going from $12.5^{\circ}$ to $15^{\circ} \mathrm{AOA}$. This is evidenced from the velocity vector plots, and the projected streamline plots. From the averaged velocity profile data, it appears that VB rapidly sweeps over the near-apex portion of the wing in this angle of attack range, rather than gradually marching upstream.

Further insights into the projection of the velocity field onto crossflow planes could be obtained by attempting to elucidate the presence of singular points from the data of Section 5.3, in the style of Figure 1.3. Unfortunately, this was generally not possible due to ambiguities introduced by the resolution of the velocity data. Also, perhaps the most interesting singular point - the saddle point above the leeward surface on the wing symmetry plane - was generally outside of the interrogation domain.

In terms of axial velocity distribution, the near-apex velocity field of the $50^{\circ}$ wing was seen to exhibit behavior consistent with conical flow. It is perhaps surprising that a wing of such low sweep would have an important quantitative flow feature usually attributed to slender wings.

The presence of secondary vortices is seen to be progressively less prominent, in going from the flow visualization to the SPIV velocity vector plots to the streamline plots. However, a structure resembling a secondary vortex was evident in the projected streamlines for the $50^{\circ}$ wing at $12.5^{\circ}$ angle of attack. 


\section{Chapter 6 Discussion}

In this chapter we consider some of the implications of the data presented in Chapters 5 and 6 . In what follows, velocity profiles in crossflow planes are presented. Vortex core trajectories are estimated, and the various results are compared to a selection of theoretical models.

\subsection{Comparison of flow visualization and SPIV results}

For both the $65^{\circ}$ and $50^{\circ}$ sweep delta wings, discernable LEV structures were shown by the flow visualization to exist down to arbitrarily low angles of attack. LEVs were documented at angles down to $2.5^{\circ}$ for both wings. These findings were supported in the PIV data, beginning with the lowest angle of attack SPIV measurements $\left(5^{\circ}\right)$. Thus the usual statement that "LEVs form on the leeward side of delta wings at sufficiently high angles of attack" is unnecessarily weak under the present conditions.

Vortex breakdown location data from the flow visualization followed trends consistent with the literature, especially above $15^{\circ}$ angle of attack. Below $15^{\circ}$, VB location for the $65^{\circ}$ wing was ambiguous, as the LEV core underwent an eventual dispersion 1-2 root chords downstream of the trailing edge, rather than any abrupt disruption in the vicinity of the wing. The PIV tests were unfortunately not sufficiently extensive to encompass conditions in which VB occurred upstream of any of the interrogation planes. Thus, breakdown conditions for the $65^{\circ}$ wing were not verified by stereo PIV. However, the main scope of this study was to consider the relatively low 
angle of attack phenomena responsible for the stall and loss of coherent vortical structures for the $50^{\circ}$ wing.

While the physics of the VB region itself was not a major focus of this study, the flow visualization revealed both spiral and bubble types of breakdown for two wings. In general the VB region and downstream flow are unsteady. For the $65^{\circ}$ wing, and for the $50^{\circ}$ wing at $10^{\circ}$ angle of attack and below, unsteadiness in and downstream of the VB region had no upstream effect on the LEV trajectories, which were steady. The spiral mode was favored for the $65^{\circ}$ wing, with the bubble mode appearing only intermittently.

The situation was much the reverse for the $50^{\circ}$ wing, where the bubble form of breakdown was the more common, and apparently a natural stage during the formation and destruction of the LEV cores in the unsteady regime from $12^{\circ}$ to $20^{\circ}$ angle of attack. In the latter case, VB progressed upstream during a LEV "destruction cycle" generally in the bubble form. In addition, a possible third form of breakdown was observed, where the vortex did not terminate in a spiraling or stagnation region, but in a whorling pattern (see, for example, Figure 4.16 and Figure 4.39). In general, the downstream extent of the LEVs was appreciably greater for the $50^{\circ}$ wing than what might have been expected from the literature. At no angle of attack was the VB location completely behind the trailing edge, though at $2.5^{\circ}$ (and to some extent at $5^{\circ}$ ) it was approximately at the TE. This contradicts some classical data, e.g., Wentz and Kohlman ${ }^{18}$.

SPIV and flow visualization had good agreement on the location and extent of the primary leading edge vortices. The strong LEVs of the $50^{\circ}$ wing were well captured by the SPIV. The characteristic angle of $15^{\circ}$, at which the flow visualization recorded at best vague indications of weak LEV-type structures, was quite consistent with the PIV, 
which showed a region of extensive flow retardation in the entire domain bounded by the wind leeward surface and the leading edge shear layer (Figure 5.56). This situation was found from the PIV data to be largely the same in the succeeding downstream interrogation planes of the PIV, beginning with the most upstream station at $\mathrm{z} / \mathrm{c}=0.118$.

Agreement between SPIV and flow visualization was not as good in the issue of resolving the secondary vortices. These were quite prominent for the $50^{\circ}$ wing in the flow visualization images (Figure 4.22 and Figure 4.24), but not observed for the $65^{\circ}$ wing. In the PIV, however, weak vortical structures were observed for $5^{\circ}-7.5^{\circ}$ angle of attack for both wings. In fact these "vortices" were difficult to distinguish from just spanwise flow, outboard of the primary LEV core. So the range of angles of attack in which these structures were found is consistent between the flow visualization and SPIV, but certainly not the extent.

Measurement of primary LEV core axial speed was also in good agreement between the SPIV and flow visualization. For the $65^{\circ}$ wing, both techniques revealed an essentially linear progression of increasing axial flow speed. At $5^{\circ}$ angle of attack, core axial speed was approximately the same as the free stream speed. By $15^{\circ}$, a noticeable "hump" in the core velocity profile could be observed. The effect was quite prominent at $20^{\circ}$ (Figure 5.27)

For the $50^{\circ}$ wing, however, the situation was quite different. Core axial velocity was approximately the same as the free stream for all angles of attack where VB was downstream of the SPIV interrogation plane. Further upstream progression of the VB region resulted in a marked decline in core axial velocity. This is discussed further in Section 6.2 below. Beyond $20^{\circ}$ angle of attack, the side view of the dye injection shows 
a separated region covering the entire planform, bounded by what looks like a shear layer with Kelvin-Helmholz waves (Figure 4.19). Such structures could in principle be detected by SPIV, but the orientation of the SPIV interrogation planes is not conducive to doing so. This is an area where a fully three-dimensional velocimetry technique would be very welcome.

\subsection{Crossflow plane velocity profiles}

With the availability of three-component velocity vector data, profiles of axial and azimuthal velocity along a $y=$ constant "cut" across the wing starboard panel can be elucidated from the data presented in Chapter 6.

Profiles of the z-component of velocity in the primary vortex cores at the $\mathrm{z} / \mathrm{c}=0.3$ station are given in Figure 6.1. In distinction with the velocity vector plots of Chapter 5, the $\mathrm{x}$ - and $\mathrm{y}$-coordinates are renormalized such that the local wingspan has extent $\mathrm{x}=1.0$ (thus the right side leading edge is at $\mathrm{x}=0.5$ ).

In the figure, as in previous instances, the distinction between core axial velocity, and the velocity component in the streamwise (z) direction, has been blurred. This is motivated by the fact that LEV primary core sweep is small, and the error introduced by equating axial to the streamwise-direction velocity component does not justify the computational effort of making the distinction.

It is seen from Figure 6.1 that the $65^{\circ}$ wing has a "jet-like" axial velocity profile in the primary core at angles of attack above $5^{\circ}$. This is modest at small angle of attack, but becomes quite pronounced by $15^{\circ}$. At $5^{\circ}$, there is a slight "wake-like profile, wherein the local axial velocity has a deficit compared to the free stream. The $50^{\circ}$ wing, however, 
has a "wake-like" behavior at every angle of attack, regardless of whether vortex breakdown is upstream or downstream of the interrogation region. When breakdown has moved upstream of $z / c=0.3$, the wake-like profile broadens, expanding further inboard. The "complete stall" description applied to the $20^{\circ}$ angle of attack case in Chapter 5 is further motivated by the supposition that de-energized flow characteristic of stall is achieved when the wake-like profiles from the left and right LEVs coalesce at the wing center plane (vertical plane of symmetry).

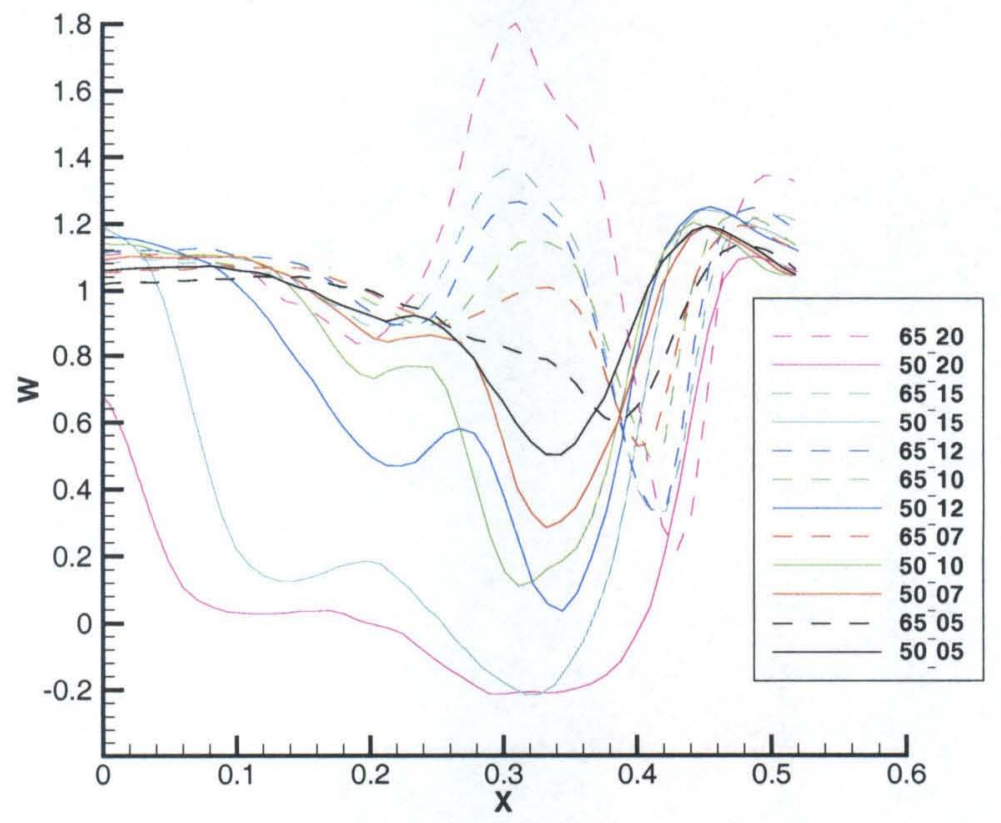

Figure 6.1. $\mathrm{LEV}$ core normalized axial velocity, $50^{\circ}$ and $65^{\circ}$ wings

Similarly, azimuthal velocity profiles are given in Figure 6.2. Azimuthal velocity, $u_{\theta}$, can be computed from the Cartesian velocity components $u$ and $v$ (the usual PIV output), by the relation $u_{\theta}=v \cos \theta-u \sin \theta$, where $\theta=\arctan (y / x)$. However, the origin of this $x-y$ coordinate system should be the LEV core. That origin will vary for each of the 12 cases presented in Figure 6.2. Note that for $\theta=0$ (to the right of the LEV center), the azimuthal polar velocity component is equal to the Cartesian $v$-component, 
and for $\theta=\pi$ (to the left of the LEV center), the v-component is the negative of the azimuthal velocity. Keeping this in mind, the v-component is plotted without a coordinate transformation.

It is seen that there is a peak of positive azimuthal velocity at the wing leading edge, where the shear layer begins its rollup process, for every test case. For the $65^{\circ}$ wing, there is the characteristic peak of opposite-signed azimuthal vorticity associated with the primary LEV core. The trend can be compared to the starboard half of the azimuthal velocity obtained from a coarse full-span image, Figure 2.13 .

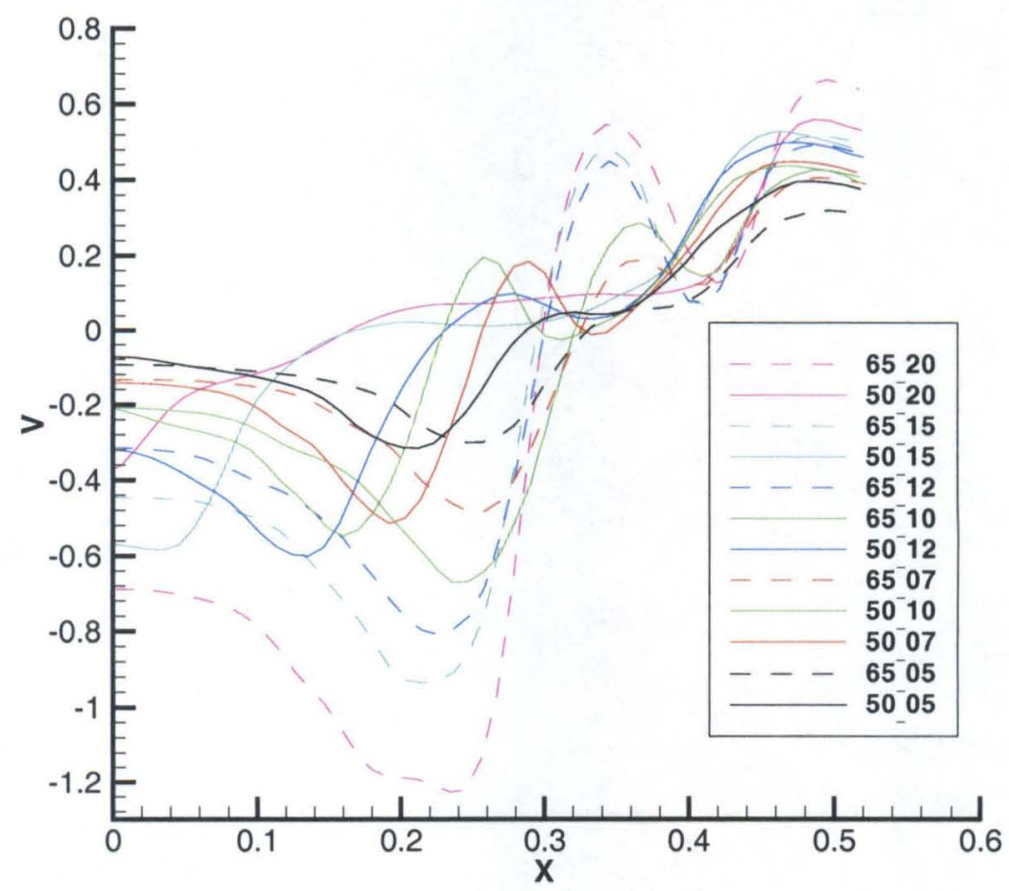

Figure 6.2. LEV core normalized azimuthal velocity, $50^{\circ}$ and $65^{\circ}$ wings

For the $50^{\circ}$ wing, the qualitative trend follows that of the $65^{\circ}$ wing up to and including $12.5^{\circ}$ angle of attack. As the angle of attack increases, data for the $50^{\circ}$ and $65^{\circ}$ wings progressively diverge. At $15^{\circ}$ angle of attack, the velocity profile for the $50^{\circ}$ wing changes abruptly, with a loss of velocity peak concomitant with the presence of VB. 


\subsection{Vortex core trajectories}

Geometry of the trajectory of the LEV, as evidenced by the location of the intersection of its core with a crossflow plane, is one of several possible measures of the distinction between a slender and nonslender delta wing. More important for the present investigation than the particular location of the LEV is the insight that measurements of the core location gives into 1) the applicability of theoretical models designed for slender delta wings, and 2) the validity of the present data collection itself.

LEV core axis location can be identified from the stereo PIV data for the two wings and the six angles of attack considered in this study. Here, "core" refers not necessarily just to the viscous portion of the LEV structure (as was the term used in Chapter 1), but to a hypothetical cylindrical tube of arbitrarily small cross section, centered about a curve defining the LEV trajectory. This curve is generally taken to be a straight line emanating from the wing apex. One criterion for identifying where LEV trajectory intersects a given crossflow plane is to consider the location of the peak axial vorticity in that plane. However, this criterion can be ambiguous when a vorticity peak is poorly defined, as is especially the case for the $50^{\circ}$ wing above $12.5^{\circ}$ angle of attack.

The criterion of peak axial velocity fails for the same reason. It is unreliable even for the pre-breakdown cases, where the peak x-component of velocity may in fact not be in the LEV at all (see Figure 6.1). Instead, the projected streamline results of Section 5.4 were used to "converge" toward the apparent center of the smallest obtainable locus of points resembling a closed streamline about the LEV core center. Locations in the crossflow plane $\mathrm{z} / \mathrm{c}=0.3$ of points corresponding to this definition of LEV core axis are presented 
in Figure 6.3. Each data point corresponds to an angle of attack value, as noted in the Figure.

It is seen that for the $65^{\circ}$ wing, the spanwise location of the cores is remarkably constant with varying angle of attack, whereas for the $50^{\circ}$ wing, the cores move inboard with increasing angle of attack. For both wings, the LEV moves further above the wing leeward side with increasing angle of attack, as is generally observed for all delta wings.

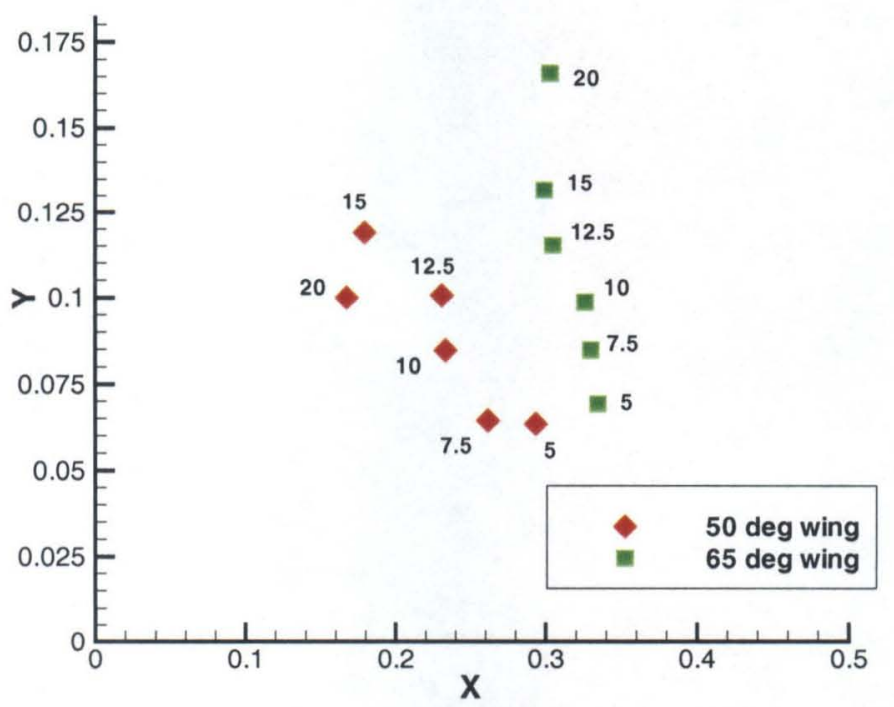

Figure 6.3. $50^{\circ}$ and $65^{\circ}$ wing $\mathrm{LEV}$ core locations, $\mathrm{z} / \mathrm{c}=0.3$

Comparison of these data to the flow visualization gives similar results, to within $20 \%$ error in the worst cases, i.e., 0.1 units of local span in the $x$-direction, in the language of Figure 6.3. The discrepancy was maximum in the post-breakdown cases, where the dye streak could not accurately track an LEV trajectory. The flow visualization results for the spanwise core location for the $50^{\circ}$ wing are plotted in Figure 6.4 below. 


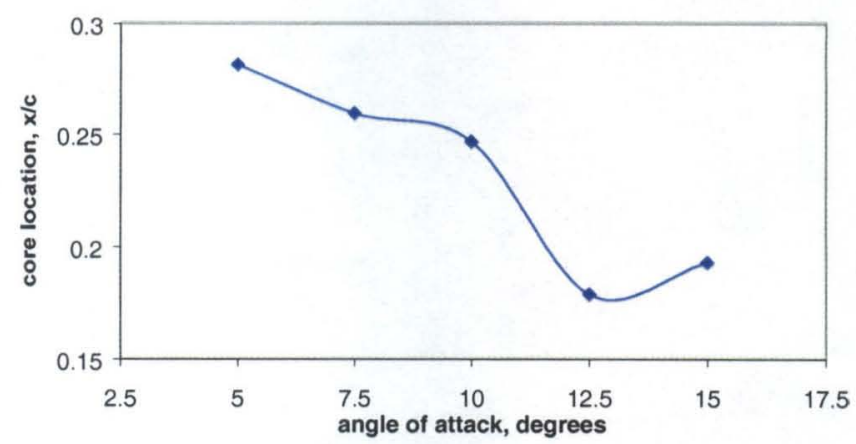

Figure 6.4. $50^{\circ}$ wing LEV core spanwise location

The core location data can also be compared to the theoretical predictions of Moore and Pullin ${ }^{28}$, who find that core location is invariant with angle of attack, and depends only on sweep angle. The predicted location of the LEV core in a given crossflow plane, as a function of wing LE sweep angle, based on model of Moore and Pullin, is shown in Figure 6.5.

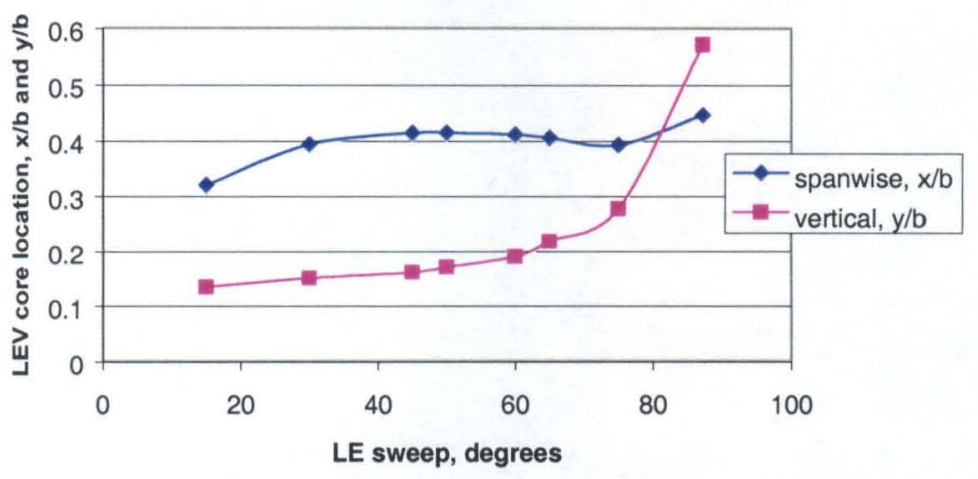

\section{Figure 6.5. Predicted LEV normalized core location vs. LE sweep}

For the $65^{\circ}$ wing, the LEV core is predicted to lie at $\sim 0.4$ local semispans outboard of the wing centerplane. The present experimental data give values of $0.3-0.35$ semispans, depending on angle of attack. The fact that the angle of attack dependency of the LEV spanwise location is weak is in reasonable agreement with the model. But for the $50^{\circ}$ wing, the predicted spanwise location is 0.41 semispans, which differs 
considerably from the above experimental data. The theoretical prediction captures the trend that the vertical location of the core will increase with increasing sweep, but the predicted values -0.22 and 0.17 semispans for the $65^{\circ}$ and $50^{\circ}$ wings, respectively -are not attained in the experimental data for the range of angles of attack under consideration. More importantly, the experimental data shows that vertical location of the core in fact varies quite strongly with angle of attack.

The slender wing model of Mangler and $\mathrm{Smith}^{74}$ considers the variation of core location with a similarity parameter that includes both the effects of angle of attack and sweep, albeit this model is expected to fail for wings of low sweep.

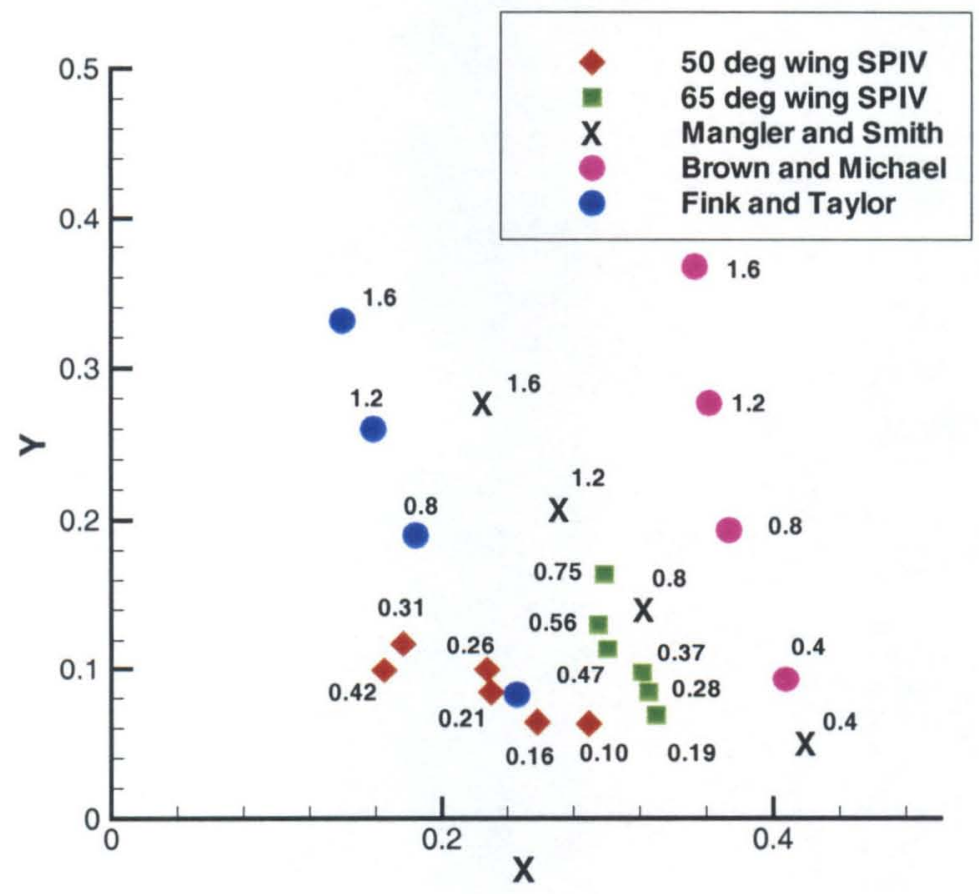

Figure 6.6. Predicted core location vs. experiment

This parameter, denoted as $K_{M S}$, is given by

$$
K_{M S}=\frac{\alpha}{\tan (\pi / 2-\Lambda)}
$$


As usual, $\alpha$ is the angle of attack and $\Lambda$ is the LE sweep angle. $K_{M S}$ increases with increasing LE sweep and increasing angle of attack. Mangler and Smith, like most researchers, consider primarily slender wings and high angle of attack, whence their results are concerned mostly with $K_{M S}$ larger than those of the present study. They give data for $K_{M S}$ values of $0.4,0.8,1.2,1.6$, and 2.0 , whereas the values of the present data set vary from 0.1 to 0.75 . Figure 6.6 is based on Figure 4 of Mangler and Smith ${ }^{74}$, with the sweep and angle of attack conditions of Figure 6.3 recast in terms of $K_{M S}$. Values of $K_{M S}$ corresponding to each data point are entered in the Figure. Also included are the theoretical model of Brown and Michael ${ }^{75}$ (also based on slender body approximations) and the high Reynolds number wind tunnel data of Fink and Taylor ${ }^{76}$ for a $70^{\circ}$ delta wing, as reported in ${ }^{74}$.

There are three issues to consider: first, whether the various data sets should be equivalent for a common value of $K_{M S}$ (which would show that $K_{M S}$ is indeed a similarity parameter), whether there is agreement between the theoretical models and experiment, and finally, whether the $50^{\circ}$ stands out as an outlying case. From the limited amount of data shown in Figure 6.6, the answers, respectively, are no, maybe, and yes. None of the five data sets in Figure 6.6 have close data points for the same value of $K_{M S}$. The model of Mangler and Smith is marginally close to the present study's data for the $65^{\circ}$ wing at the higher angles of attack, but not for other conditions. Core locations for the $50^{\circ}$ wing lie far inboard of all of the other data sets. Fink and Taylor's data point at $K_{M S}=0.4$ appears amidst the $50^{\circ}$ wing data points, but the $K_{M S}$ value of the latter is much smaller. 
The general trend that the experimentally determined location of the primary LEVs is further inboard than that of the theoretical predictions is captured in the present case as well.

The more refined model of Smith ${ }^{77}$ considers the core location as a function of a slightly revised sweep/incidence similarity parameter, given as

$$
a_{s}=\frac{\tan \alpha}{\tan (\pi / 2-\Lambda)}
$$

Smith's prediction gives values of core spanwise location that are rather too far outboard of those of the present data set. This trend is seen in Figure 6.7, where the spanwise locations of the starboard LEV cores are again plotted for the two wings, this time vs. $a_{s}$.

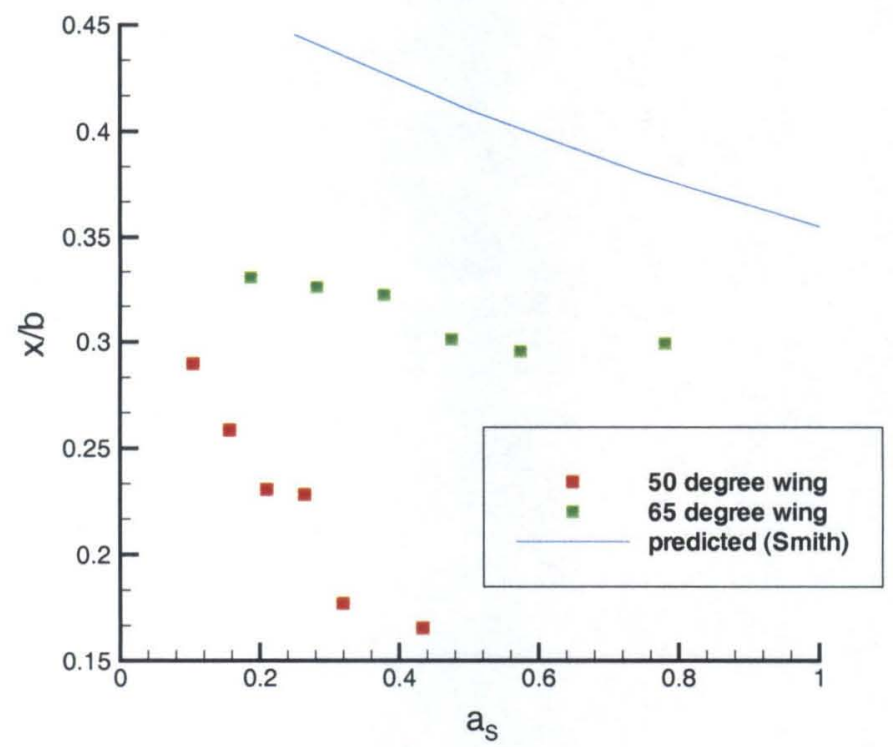

Figure 6.7. Comparison of normalized spanwise core location with model of Smith ${ }^{77}$

As expected, the data for the $65^{\circ}$ wing fit the model much better than do the $50^{\circ}$ wing data. In particular, the former have a slope vs. $a_{s}$ comparable to the model's prediction. It should be mentioned that Smith's model assumes the existence of discrete windings of 
the LE vortex sheet, wherein the vortex sheet winds about the LEV core axis for many revolutions, before finally spiraling into a thin core. This assumption is consistent with Reynolds number approaching infinity, and not surprisingly contradicts the conjectures stated in the present work; namely, that the LE shear layer makes at most a single revolution before being subsumed in a smeared, viscous rotating flow. This is a basic consequence of low Reynolds number. Quantitative discrepancy between the present data and Smith's predictions can therefore at least in part be attributed to Reynolds number effects.

Using slender body concepts inspired by the same notion of similarity parameter, Huang and Chow $^{78}$ considered a stability analysis of LEVs over a slender wing, in the sense of normal modes. The LEVs were subjected to a small displacement, and the sign of the real portion of the eigenvalues of the disturbance growth were calculated. These were found to be negative for all values of $a_{s}$, but increasing in magnitude with decreasing $a_{S}$. Curiously, this implies that for a given angle of attack, the LEVs of the less slender wing are more stable. But one must approach the notion of stability with some discretion. As Huang and Chow point out, their model assumes that crossflow plane gradients dominate streamwise gradients (thus, the slenderness assumption is approached in the second sense of Section 1.5.1), whence the analysis is only relevant for relatively large values of $a_{s}$ for any reasonable angle of attack. Also, instability due to breakdown is not considered.

\subsection{Reynolds number effects}


With the sole exception given in this section, all of the data in the present work were taken at a free stream flow speed of $80 \mathrm{~mm} / \mathrm{s}$. The following, however, were taken at $32 \mathrm{~mm} / \mathrm{s}$, corresponding to a Reynolds number of 6200 based on root chord. This is the case of the $65^{\circ}$ wing at $\alpha=15^{\circ}$.

Mean velocity vector field data, again at $\mathrm{z} / \mathrm{c}=0.3$, are shown in Figure 6.8 and Figure 6.9. These can be compared to Figure 5.26 and Figure 5.27.

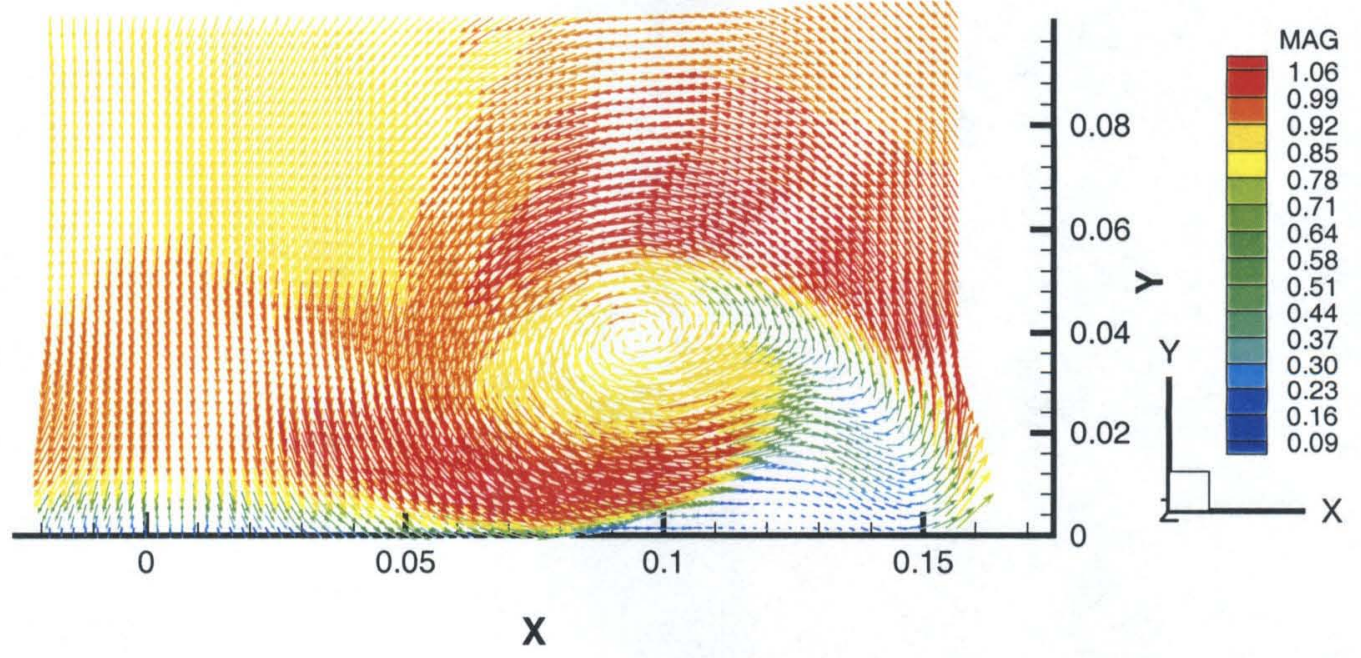

Figure 6.8. $65^{\circ}$ wing, $\alpha=15^{\circ}$, mean velocity field
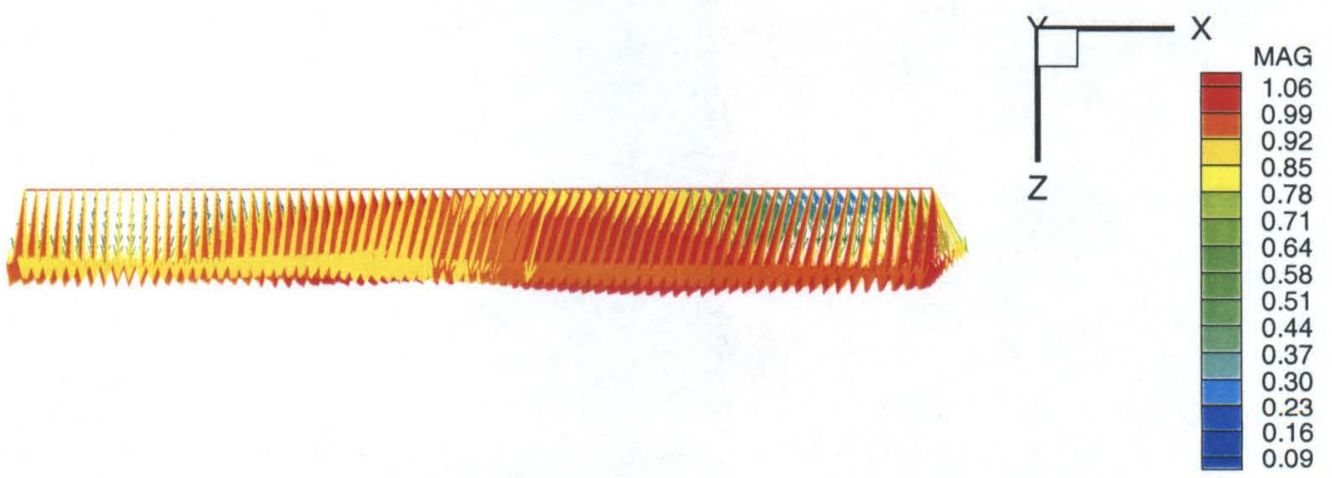

Figure 6.9. $65^{\circ}$ wing, $\alpha=15^{\circ}$, mean velocity field, top view

Evidently, even when comparing one small Reynolds number to another, there are still important distinctions. In going from $\mathrm{Re}=15400$ to 6200 , the size, shape, and location of the primary LEV does not appear to vary significantly in the streamwise view. But, the axial velocity 
profile, as seen in the top view, is entirely different. The higher Re case displays the relatively high LEV core axial velocity, which, as pointed out in Chapter 6, is in fact the usual case. The Re $=6200$ case, however, has no discernable axial velocity peak in the LEV core. In addition, the $\operatorname{Re}=6200$ case has what appears to be a stronger secondary vortical flow outboard of the primary LEV - whereas the higher Re case has what was termed a "stagnation region." Perhaps paradoxically, the lower Re case reverses the supposed trend in increasingly more stagnant outboard flow in going towards lower Reynolds number.

The situation is seen more closely by considering the axial and azimuthal velocity profiles for the two Reynolds number cases (Figure 6.10).

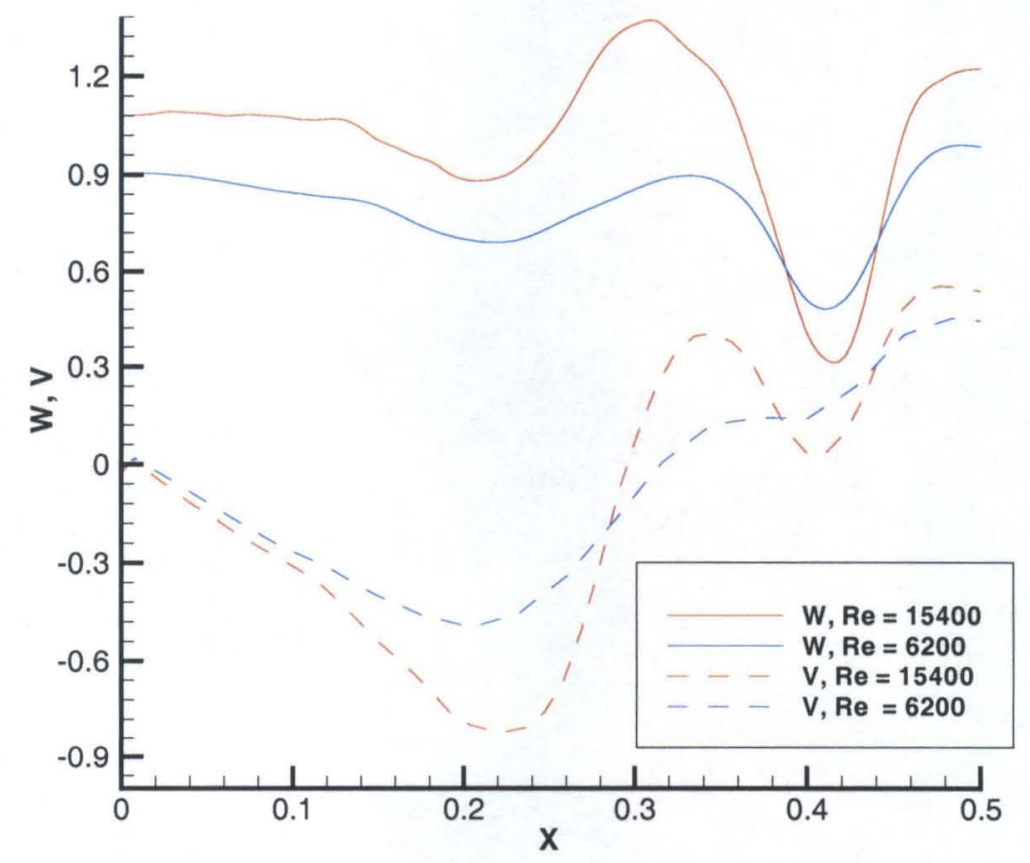

Figure 6.10. $65^{\circ}$ wing normalized velocity profiles for two different Reynolds numbers

Traub $^{79}$ considered Reynolds number effects in the $20,000-60,000$ range for wings of $60^{\circ}$ and $70^{\circ}$ sweep. At $\mathrm{z} / \mathrm{c}=0.6, \alpha=17^{\circ}$ and $\mathrm{Re}=60,000$, leeward-surface static pressure data showed the usual peak in $-C_{P}$ at the primary LEV core, followed by a slight pressure recovery, then a local suction peak in the secondary LEV core, and then 
another rise in going outboard toward the leading edge. But for $\operatorname{Re}=20,000$, the pressure peak at the primary LEV was weak, and $C_{P}$ was essentially constant in going further outboard, missing entirely the presence of a secondary vortex. Evidently, there is a fundamental change in the flowfield in going from $\operatorname{Re}=20,000$ to 60,000 . For the wings of the present experiment, at $\operatorname{Re}=15,000$ (65 $5^{\circ}$ wing) and 8000 (50 wing), the general situation is similar to that observed by Traub at $\operatorname{Re}=20,000$; there is a suction (as evidenced by higher flow speed in the LEV than in the free stream) in the primary LEV, but the outboard flow is stagnant. But then, for the $\operatorname{Re}=6200$ case for the $65^{\circ}$ wing, the situation appears to change yet again; suction in the primary LEV is very weak, but the outboard flow is somewhat less stagnant. Measurements over a range of Reynolds number from 5000 to 20,000 and above would help to elucidate the Reynolds number dependency with more rigor.

On the other hand, a broad collection of wind tunnel results suggests that for Reynolds numbers of $O\left(10^{5}\right)$ and beyond, Re effects are indeed small. For example, Re effects on leeward surface pressure coefficient data are considered by Roos and Kegelman ${ }^{80}$ for wings of $60^{\circ}$ and $70^{\circ}$ leading edge sweep.

\subsection{Circulation contour plots}

Circulation can be computed for the velocity data, by taking planar projections of the SPIV data (thus, essentially reverting to 2-D DPIV data). The vortex core locations from Figure 6.3 were used as center points of circular contours of various radii, $R$, along which the line integral of velocity was computed. Varying this radius from zero to some common cutoff threshold resulted in the family of curves in Figure 6.11. Again, the 
station $\mathrm{z} / \mathrm{c}=0.3$ was selected. In Figure 6.11 , the left-hand vertical axis shows circulation values normalized by the free stream velocity and the wing trailing edge span ( $180 \mathrm{~mm}$ for both wings). The right-hand vertical axis shows an alternative normalization of circulation by the kinematic viscosity; in this case, $1 \times 10^{-6} \mathrm{~m}^{2} / \mathrm{s}$. The latter is useful for considerations of the extent to which the LEV is turbulent.

From Figure 6.11, we identify five trends:

1) In general, as the angle of attack is increased, the characteristic radius of the LEV (defined as the radius at which peak circulation is achieved) increases. This broadening of the LEV viscous core is especially clear for the $65^{\circ}$ wing, in going from $5^{\circ}$ to $20^{\circ}$.

2) With increasing angle of attack for a given wing, circulation also increases, until breakdown approaches the cross-sectional station where the circulation was measured.

3) Peak circulation is lower in a post-breakdown situation. That is, if the angle of attack is increased to the point where breakdown crosses the station where the circulation was measured, that measurement will be lower than it was for the lower angle of attack, when breakdown was further downstream.

4) For any given angle of attack, the LEVs of the $65^{\circ}$ are both broader and stronger (i.e. have more circulation) than those of the $50^{\circ}$ wing.

5) Circulation curves for the two wings are similar at low angle of attack, and progressively differ more with increasing angle of attack. 


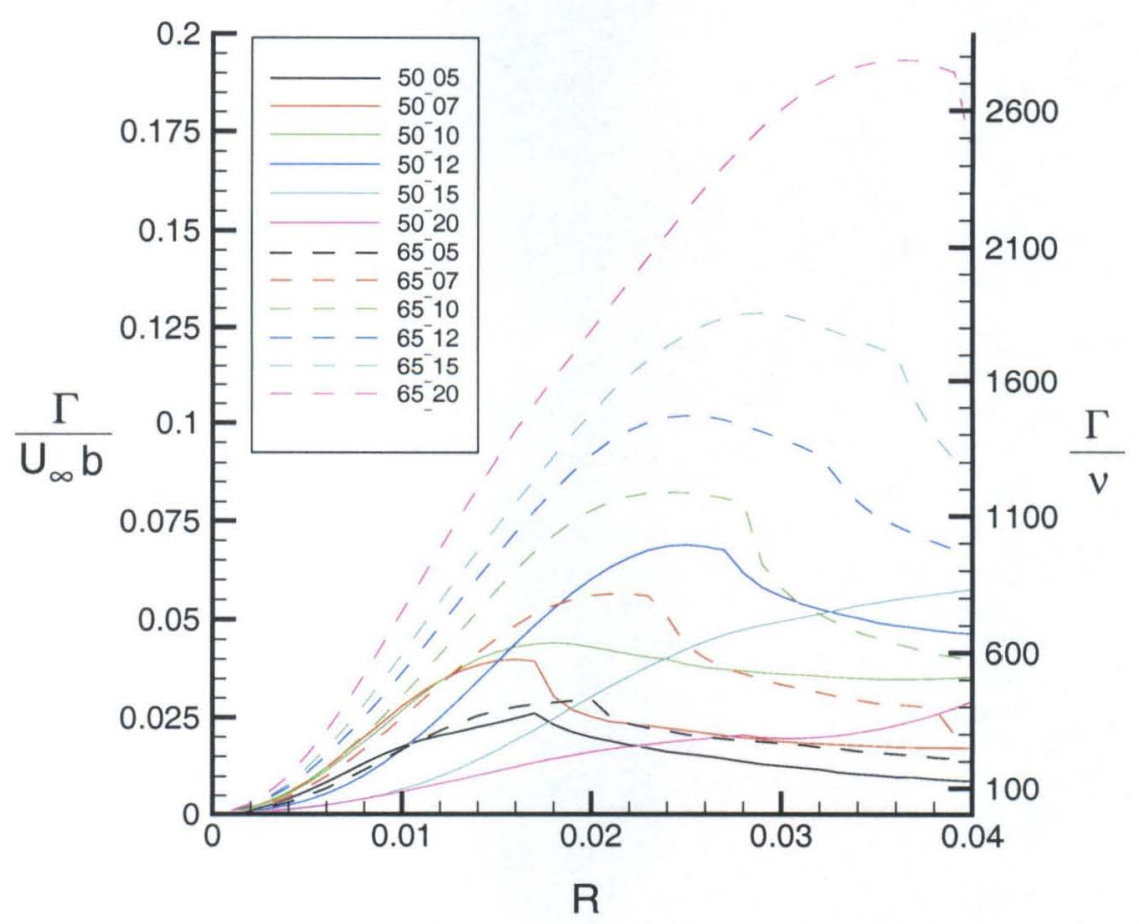

Figure 6.11. Circulation vs. vortex radius

The circulation curves for the $50^{\circ}$ wing show convincing evidence that breakdown crossed the $\mathrm{z} / \mathrm{c}=0.3$ station somewhere between $12.5^{\circ}$ and $15^{\circ}$ angle of attack. The curve for the former angle has a definite circulation peak, whose size and radius follows the trend in going from the smaller angles of attack. The $15^{\circ}$ curve, however, shows no clear peak, and a lower peak circulation. The $20^{\circ}$ curve shows a much lower peak.

It should also be noted that for the lower angles of attack, and especially for the $50^{\circ}$ wing, the use of circular contours of integration for the computation of vorticity might not be the best choice. LEVs for these cases tended to be more elliptical in cross sectional shape, with the long axis of the ellipse in the spanwise direction. For radii greater than the distance between the LEV center and the wing leeward surface, the 
circulation computation can no longer be trusted. In particular, the computed value of circulation appears to decrease at such radii.

The fact that the peak circulation value is only attained at a radius comparable to the distance from the LEV center to the inner side of the LE shear layer is an indication that this entire region is viscosity-dominated. In other words, at the Reynolds number of the present investigation, the "windings" of the shear layer can not be resolved into individual vortex sheets. The vorticity is diffused to the point where one can speak of the LEV core and the LEV itself almost interchangeably, at least in terms of the velocity components in crossflow planes.

Interestingly, the normalized axial velocity evaluated at the core radius which corresponds to the peak circulation, $R_{0}$, is rather smaller than 1.0. The classical result for delta wings is that the axial velocity at this radius should be comparable to free stream, or slightly greater. In fact, the axial flow data in Figure 6.1 are not symmetric about the core axis, when taken at a radius $R_{0}$. Going a distance $R_{0}$ to the left of the LEV center produces a different axial velocity than going $R_{0}$ to the right. In Figure 6.12, the average values of the axial velocity for the two wings at $R_{0}$ are plotted for each angle of attack. Values of $R_{0}$ for each angle are given in the same plot, as fractions of local semi-span. 


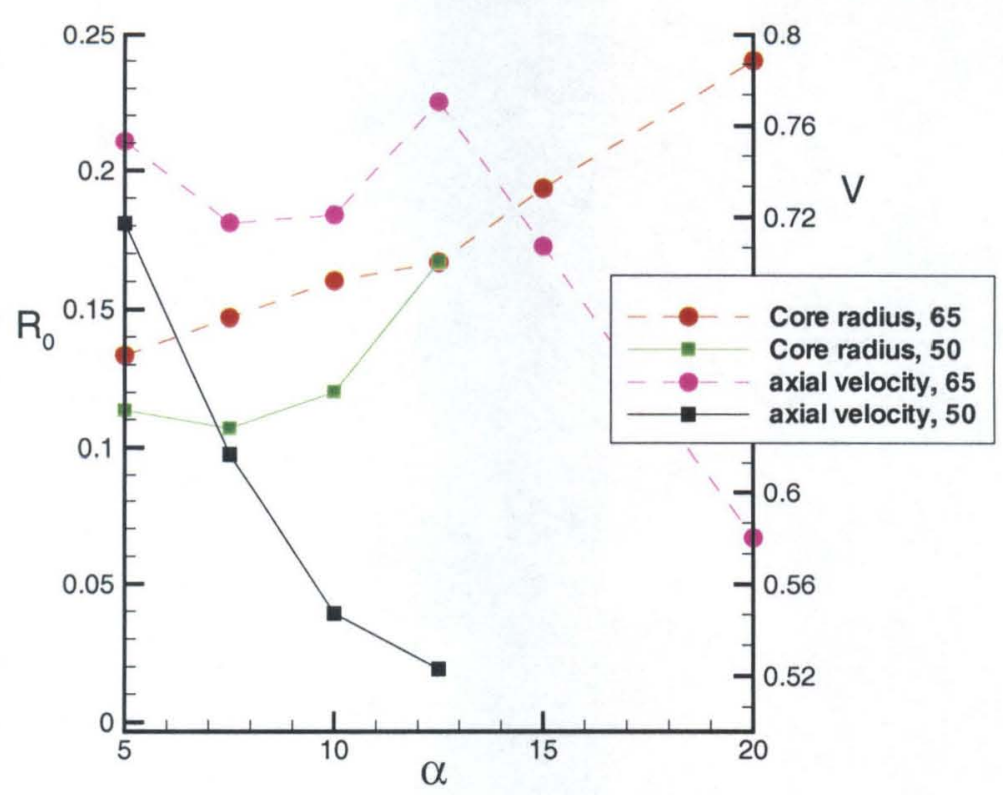

Figure 6.12. Averaged normalized core velocity and LEV radius vs. AOA

Core velocities and radii for the $50^{\circ}$ wing at $15^{\circ}$ and $20^{\circ}$ angle of attack are not shown, since stable LEVs could not be defined at these conditions.

\subsection{Comparison with an LEV stability model}

The velocity profile results of Section 6.2 and the circulation results of Section 6.5 can be combined to make some statements about the characteristic primary LEV core diameter, the core edge swirl ratio, and the "profile parameter" in the sense of Wedemeyer $^{34}$ (see Section 1.6). Values of average axial and azimuthal velocities at a distance $R_{0}$ from the LEV center can be combined to form an estimate of the profile parameter, $K_{W}$, defined by Wedemeyer as

$$
\frac{\sqrt{K_{W}+0.5}}{K_{W}}=\left.\frac{U_{\theta}}{U_{Z}}\right|_{R=R_{0}}
$$


$K_{W}$ is essentially just another measure of the helix angle of the LEV. Solving for $K_{W}$ (and taking the positive solution of the resulting quadratic), one obtains the values in Table 6-1. Beyond $15^{\circ}$ angle of attack, values for the $50^{\circ}$ wing could not be computed because of the absence of a coherent LEV. Comparing the obtained values of $K_{W}$ with those of the stability diagram in Figure 1.14, there is a stunning and fortuitous agreement between the predicted LEV stability threshold at $K_{W}=1.16$ and the highest obtained value of $K_{W}$ for a stable LEV for the $50^{\circ}$ wing. However, the small values of $K_{W}$ for the $65^{\circ}$ wing at $15^{\circ}$ angle of attack and beyond would have suggested that the LEVs would no longer be stable, which is clearly not the case. The explanation is, first, that the choice of abscissa for the evaluation of the axial and azimuthal LEV core velocities is a matter of some ambiguity, and second, that the comparatively low values of core peak axial velocities result in particularly low values of $K_{W}$.

\title{
Table 6-1. Evaluations of Wedemeyer's profile parameter
}

$\begin{array}{ccc}\text { AOA } & \begin{array}{c}\mathbf{6 5} \text { degree wing } \\ \text { K_w }\end{array} & \begin{array}{c}\mathbf{5 0} \text { degree wing } \\ \text { K_w }\end{array} \\ 5 & 7.15 & \\ 7.5 & 2.61 & 7.79 \\ 10 & 1.53 & 1.96 \\ 12.5 & 1.28 & 1.42 \\ 15 & 0.92 & 1.15 \\ 20 & 0.50 & \\ & & \end{array}$

\begin{abstract}
A better criterion than just "radius of peak circulation" is needed for deciding where to evaluate the axial and azimuthal velocities, but that this is arbitrary, and invites subjective manipulation. The difficulty can again be attributed to the smearing effects of low Reynolds number.
\end{abstract}


Further insight into the comparison between the $50^{\circ}$ and $65^{\circ}$ wings can be gained by considering some relative measure of the LEV strength. Hemsch and Luckring ${ }^{21}$ give the normalized vortex strength as a function of LE sweep and root chord, $c$, as

$$
\frac{\Gamma}{U_{\infty} c} \sim \frac{\cos \alpha(\tan \alpha)^{1.2}}{(\tan \Lambda)^{0.8}}
$$

for fixed angle of attack. Evidently, LEV strength increases with decreasing sweep. However, for the delta wings in the present experiments, trailing edge span was held constant while root chord was decreased to decrease sweep. Rewriting this relation for constant trailing edge sweep, $b$, one obtains

$$
\frac{\Gamma}{U_{\infty} b} \sim \cos \alpha(\tan \alpha)^{1.2}(\tan \Lambda)^{0.2}
$$

Then, the left-hand side eqn. 7-4 divided by right-hand side should be a constant across varying sweep and angle of attack. This quantity is plotted in for the $50^{\circ}$ and $65^{\circ}$ wings and the six angles of attack investigated in this study. It is "roughly" constant, around 0.5 , for both wings and all angles of attack, except for the obvious case were the LEVs for the $50^{\circ}$ wing have undergone breakdown. 


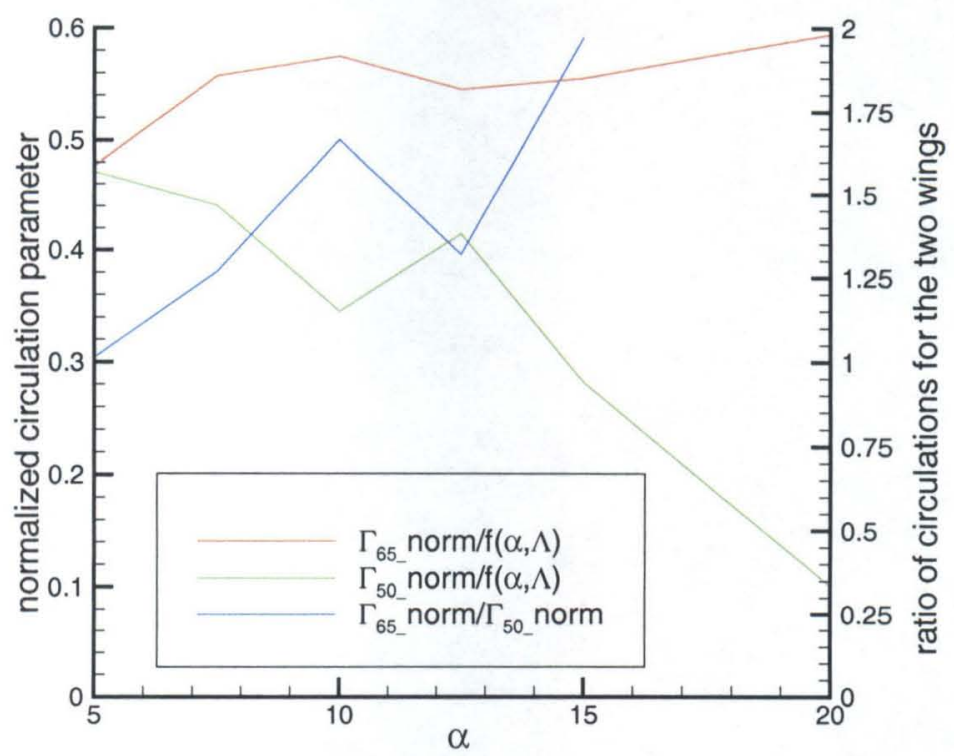

Figure 6.13. LEV strength vs. Hemsch and Luckring prediction

For each angle of attack, the ratio of the left hand terms of eqn. 7-4 evaluated for the $65^{\circ}$ wing, divided by that evaluated for the $50^{\circ}$ wing, is also be plotted vs. angle of attack. If the result is a constant, then the sweep dependency in eqn. 7-4 would be shown to be reasonable. Again, up until breakdown, a regard of the dependency as a constant is not unreasonable. The dependency of vortex strength on sweep should therefore be treated with some care. In the present investigation, the LEVs of the $50^{\circ}$ wing were clearly weaker than those of the $65^{\circ}$ wing, without contradicting the result of Hemsch and Luckring.

\subsection{Secondary vortices}

The stereo PIV data generally show regions outboard of the primary LEVs to be stagnation-type zones for angles of attack beginning with $10^{\circ}\left(50^{\circ}\right.$ wing) or $12.5^{\circ}\left(65^{\circ}\right.$ wing). Below these angles of attack, an organized motion outboard of the primary LEVs 
and inboard of the leading edges was visible, but this was not convincingly identified as vortical flow.

Here it is worth mentioning the importance of the secondary flow, especially at low Reynolds number. Numerous authors in the literature (e.g., Hummel ${ }^{81}$; more recently, Shih and Ding, using 2D PIV) point out that in a laminar flow, the secondary vortices are larger than in the more turbulent, higher Reynolds number case. This is reasonable, since the secondary flow is largely a boundary layer phenomenon, caused by separation due to an adverse spanwise pressure gradient along the boundary layer. Laminar boundary layers are of course more sensitive to such gradients. The larger secondary flow is expected to have more effect on the primary vortical flow, either by displacing its trajectory, or affecting its strength, or possibly participating in some mechanism of instability.

It is intuitively tempting to make a conjecture on the role of the disappearance of the secondary vortices prior to the onset of breakdown of the primary vortices. This disappearance would be part of the transition to imbalance between vorticity generation and transport. However, the cause and effect relationship is unclear at best; secondary vortices form due to the presence of the primary vortices, and not vice versa.

\subsection{Boundary layer effects}

Viscous effects such as the shear layer thickness can be considered by first estimating the local boundary layer thickness. A crude estimate of the windward-side boundary layer thickness immediately before the leading edge can be obtained by considering the flow from the windward-side node of attachment toward the LE to be that of a flat plate, and applying the Blasius flat plate formulas ${ }^{83}$. This is essentially 
equivalent to supposing that there is no spanwise pressure gradient on the windward side. The windward attachment node is assumed to be near the apex, whence the effective "flat plate distance" is just the distance from the apex. This conjecture is supported by the onsurface flow visualization of Su et al. ${ }^{22}$, for example. For the Reynolds numbers under consideration, $O\left(10^{4}\right)$, the boundary layer is assumed to be laminar.

The estimated windward-side boundary layer displacement thickness is the usual square root dependency, given in Figure 6.14, according to

$$
\delta^{*}=\frac{1.72 x}{\sqrt{\operatorname{Re}_{x}}}
$$

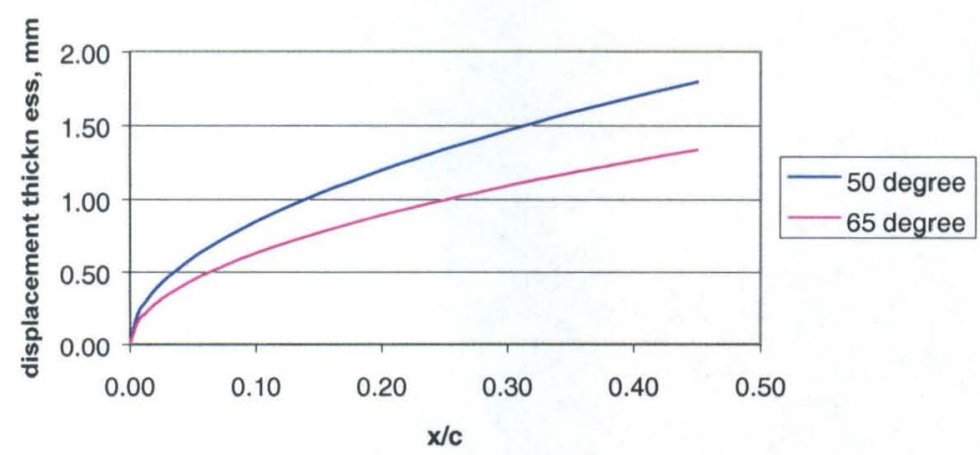

\section{Figure 6.14. Estimated windward boundary layer displacement thickness}

Thus, again at the $\mathrm{z} / \mathrm{c} \sim 0.3$ streamwise station, the boundary layer just prior to separation and rollup has a nominal displacement thickness of about $1-1.5 \mathrm{~mm}$, or 0.007 units of trailing edge span, or about $4 \%$ of the local semi-span. This is seen to be approximately the same as the resolved shear layer thickness over the leeward side. For example, the shear layer in Figure 5.62 is approximately $5 \%$ of the local semi-span in thickness. This value was arrived at by inspection of the distance across which the velocity magnitude gradient is appreciable. 
Apart from supporting the claim that primary viscous effects were reasonably well resolved by the SPIV, the result of Figure 5.62 shows that the shear layer rollup is preserved even while the LEV vortex has evidently undergone breakdown.

\subsection{Some conjectures regarding the $50^{\circ}$ wing}

The more extensive presence of coherent primary LEVs for the $50^{\circ}$ wing, compared to what was observed in some prior experiments, was probably due to auspicious bevel geometry and low Reynolds number effects. Unfortunately, data for the $50^{\circ}$ wing at the lower test speed of $32 \mathrm{~mm} / \mathrm{s}$ were not available, so it was not possible to determine whether the "low Reynolds number effect" is strengthened at yet lower speeds.

The eventual destruction of the LEVs is still caused by breakdown, but the interaction between each LEV and its neighboring secondary vortex and the leeward boundary layer strongly affects the nature of the breakdown. Unsteadiness in the LEVs is a transitional phase analogous vaguely to classical airfoil stall. It is quite distinct from high angle of attack unsteadiness of very slender delta wings (called "wing rock"), or the very high angle of attack unsteadiness observed by Ayoub ${ }^{25}$.

The unsteadiness is caused by a loss of the balance between vorticity creation and vorticity convection (following an argument such as that of Lee and $\mathrm{Ho}^{82}$ ). As one leading edge structure becomes super-saturated with vorticity, it "erupts," causing upstream propagation of the apparent location of VB. Then, the relationship is exchanged with the flow structure associated with the opposite leading edge. The details of the left-right interaction are unclear from the FV, and have not been answered by the PIV because in an effort to improve spatial resolution, the experiments only focused on one of the LEVs, and not both simultaneously. 


\subsection{A casual resume of sources of error}

Having considered a protracted exposition of the SPIV data, some further comments on the accuracy of the data are appropriate. Errors associated with DPIV in general, and stereo PIV in particular, were mentioned in Chapters 2-4. Here it is worth repeating that the two main problems with PIV applied to the delta wing flow field are those of spatial resolution in the bulk flow field, and particular inaccuracies in the nearwall flow field. The former can (perhaps simplistically) be reduced to the limited pixel resolution of digital cameras, whereas the latter are due to optical effects (such as reflections and scattering of light off the wing surface). There is also the more general error of parallax-induced spurious in-plane velocities due to out-of-plane velocity.

The actual magnitude of parallax errors is difficult to gauge, especially in the absence of an alternative velocimetry technique. However, casual inspection of the free stream "proof of concept" images suggests an upper bound to velocity measurement error of about $8 \%$; that is,

$$
\frac{\left|\vec{u}_{\text {meas }}-\vec{u}_{\text {true }}\right|}{\vec{u}_{\text {true }}}<0.08
$$

for the worst case, and rather less for most interrogation windows. This is not accurate by engineering testing standards. Whether this is nevertheless acceptable for a conceptual study is of course a matter of opinion.

Limits of PIV measurement resolution are especially significant in high-gradient flow areas, such as the LE shear layer and leeward-surface BL. Again referring to Figure 5.62, one can compare the Piv interrogation window size and the shear layer thickness. 
The former is $32 \times 32$ pixels, which scales to about $5 \%$ of the wing semi-span at that crossflow plane. Window-to-window offset is $8 \times 8$ pixels; this is the quantity that corresponds to the spatial density of the computed velocity vectors. Thus, window size is comparable to shear layer thickness, which window offset allows for some coarse resolution of shear layer structures. Comparison with the corresponding vorticity contour plot, Figure 5.88, shows vortical structures in the shear layer with an apparent diameter of about $3 \%$ of the local semi-span.

Other sources of error are more "fluid mechanical." These include water tunnel issues: test section blockage, vibration and upstream influence of the mirrors, and flow nonuniformity and turbulence in the test section.

Due to the low angles of attack involved, blockage was rather small. The worst case was the $65^{\circ}$ wing at $20^{\circ}$ angle of attack, for which the blockage ratio was $2.2 \%$. No attempt was made to correct the velocity data for blockage, or to account for the possible stabilizing effect on the LEVs by the test section walls.

\subsection{Summary}

Figure 5.62 can now be considered in light of the comments made in Section 1.5.3. Namely, the leading edge Kutta condition can not be invoked if the flow separating at the leading edge does not eventually reattach. Such is the situation in Figure 5.62. The failure of the LE shear layer to reattach at the wing leeward surface can intuitively be considered to be a symptom (if not the cause) of the loss of aerodynamically favorable leading edge separation, in the context of ${ }^{8}$. 
The observed trends in circulation, vortex core location, breakdown location, and so forth, can justifiably be considered reasonable. This is confirmed by comparison with a selection of theoretical and phenomenological models, though there are considerable ambiguities in the treatment of the experimental data, principally due to the strong effects of viscosity and in particular the stagnation region outboard of the primary LEV.

Since the selection of experimental and theoretical data in the literature is profusely broad, the present choice of comparison data is necessarily cursory. However, one can conclude that the $65^{\circ}$ wing shows trends - for example, those of the LEV core location - to be in nominal accord with the predictions of slender-wing theoretical models, whereas the $50^{\circ}$ wing is more of an outlying case. 


\section{Chapter 7 Conclusions and Further Work}

"...The rest of the proof is left as an exercise for the grader"

-Anonymous

\subsection{Some general comments}

It has been wryly but accurately observed by numerous investigators of the delta wing flowfield, that the only thing simple about delta wings is their shape. Indeed, the breadth of the literature, but the continued vagueness and appearance of isolated new facts in numerous topics of leading edge vortex stability, surface flow, structures in the leading edge shear layer, etc., all attest to this fact.

The present investigation has been carried out as a conceptual study, in large measure as a proof-of-concept of the application of stereo PIV to a complex flow of aerodynamic interest, conducted in a water tunnel environment. With the establishment of this proof of concept, and the concomitant realization of the limitations of the technique, one can proceed to more systematic investigations of the various parameters that may be suspected to significantly affect the flowfield of the delta wing. Some of these are suggested below.

With the dominant flow direction normal to the laser light sheet, alignment and displacement of the light sheet is a common problem in PIV, stereo or otherwise. The problem is compounded by adverse optical effects of working near a solid boundary (the wing surface). Low speed flow, use of fluorescent particles of diverse specific gravity, and streamwise stepping of the light sheet contributed to a reasonable solution of these problems. Use of mirrors placed in the test section behind the model, while an awkward approach to solving the optical problems of stereo PIV in a multiple optical interface 
environment, allowed for robust data collection at the low speeds involved. This was made possible by a new, quiet water tunnel constructed specifically for this experiment.

Stereoscopic PIV gives interesting insights into the strongly three-dimensional flowfield over the leeward side of a nonslender delta wing. In particular, one simultaneously obtains information about 1 ) the axial and azimuthal velocity in the leading edge vortex cores, and 2) velocity in the leading edge shear layer and the region bounded by the primary leading edge vortex and the shear layer. However, it should be kept in mind that assembly of SPIV data in succeeding downstream planes is laborious, and the presentation of the resulting three-dimensional data suffers from the usual encumbrances of attempting to work in 3D. Furthermore, SPIV is not a fully 3D technique.

It should be noted that at the low Reynolds numbers of the present investigation, the flowfield about the leeward side of the delta wings was fully viscous, in contradistinction to the generally accepted situation at high Reynolds number, where viscous effects are limited to small regions (the LEV subcore and the LE shear layer). The spatial extent of viscous effects complicates the extension of the present results to realistic applications in aeronautical design. It makes it difficult to isolate the effects of leading edge sweep.

\subsection{Summary of flow visualization results}

Off-surface flow visualization by the standard technique of dye injection into the windward stagnation region revealed the presence of strong, coherent LEVs for the $50^{\circ}$ wing, at angles of attack from $2.5^{\circ}$ to $10^{\circ}$. Prominent secondary LEVs were also 
observed in the flow visualization. The secondary vortices were observed to lose their strength earlier than the primary vortices; at $10^{\circ}$ angle of attack, the secondary vortices are almost too weak to observe, whereas the primary vortices maintained their coherence upstream of clearly defined breakdown regions, at approximately $z / c=0.7$. At higher angles of attack, large-scale instability of the primary vortices was observed. Breakdown upstream/downstream propagation was exchanged from the left to the right semispans, with a characteristic period on the order of $\sim 7$ convective times, but without obvious evidence of periodicity. By $20^{\circ}$, the flow was entirely "stalled," with no evidence of any stable vortical structures in the flowfield over the wing planform.

For the $65^{\circ}$ wing, stable LEVs were also observed for very low angles of attack, but were present all the way to $25^{\circ}$ and beyond. Below $15^{\circ}$ angle of attack, there is no observable VB at all. Rather, the LEV trajectory abruptly turns at the trailing edge from a "conical" pattern over the wing, to a direction aligned with the free stream; but the dye trace following the vortex core continues. Above $15^{\circ}$ angle of attack, the primary leading edge vortices were subject to an organized, steady upstream progression of breakdown with increasing angle of attack, with no evidence of unsteadiness upstream of breakdown. Flow visualization did not reveal secondary vortices comparable to those found for the $50^{\circ}$ wing.

\subsection{Summary of stereoscopic particle image velocimetry results}

Stereoscopic particle image velocimetry confirmed the presence of LEVs for both wings. Unfortunately, the mostly stagnant regions outboard of the primary LEV, as revealed by the stereo PIV, make difficult any assessment of the role of the secondary 
vortices, which is conjectured to otherwise have been significant for a nominally laminar flow present at the low Reynolds numbers of this study. The presence of low-speed and even reverse-flow regions outboard of the primary LEV is consistent with some other recent experiments. At moderate angles of attack, some evidence for secondary vortices was found, but certainly not to the extent as in the flow visualization.

The $50^{\circ}$ wing, while displaying some marked differences from the $65^{\circ}$ wing, still has appreciable LEV-type flow. Its role as a true limiting case of delta wing leading edge vortical flow is questionable, despite the fact that most previous experiments have shown appreciably less prevalent LEVs over the planform of a $50^{\circ}$ wing. These also show that that the choice of LE shape is especially significant for wings of moderate sweep. The windward bevels used in the present experiment are evidently the most conducive shape to LEV formation.

Whether or not the $50^{\circ}$ wing is also a transitional case between the stall behavior of slender delta wings and the classical "higher aspect ratio" wings is also a matter of interpretation. The $50^{\circ}$ wing was found to "stall" rather abruptly, in terms of upstream vortex breakdown procession with increasing angle of attack. And the lack of high speed axial flow so characteristic of slender delta wing LEV cores is more reminiscent of the separated flow of unswept wings than of classical delta wings. Of course, the issue of stall concerns the behavior of lift and moment coefficients, as well as that of the flowfield kinematics, and the former were not measured in this study.

For slender wings, the LEV cores are a location of very high speed axial flow. With increasing angle of attack, the LEV core axial speed for the $65^{\circ}$ wing increased linearly, reaching $\sim 2.5$ times the free stream speed. But for the $50^{\circ}$ wing, LEV core axial 
speed never appreciably exceeded free stream speed, and abruptly decreased downstream of the breakdown region.

At the lowest angle of attack, velocity profiles, LEV circulation, and qualitative assessment of velocity vector geometry indicated that the flow over the $50^{\circ}$ and $65^{\circ}$ wings was not drastically different. However, the distinction between the two wings increased progressively with increasing angle of attack.

\subsection{The passage toward stall for the $50^{\circ}$ delta wing at low Reynolds number}

Leading edge vortices are coherent, organized structures, which produce suction over the wing leeward surface. As such, in the presence of LEVs, the flow about the leading edges is separated, but not stalled. "Stall" of the wing, then, refers to conditions where 1) the LEVs are in a post-breakdown state over most of the wing, 2) there are no appreciable remaining regions of attached flow (with coherent LEVs, the region along the leeward side inboard of the primary LEVs generally has attached flow), and 3) the trailing edge Kutta condition is no longer present. Condition (3) was not verified in the present investigation, but regarding the first two conditions, the passage toward stall for the $50^{\circ}$ delta wing at low Reynolds number can be described as follows.

Vortex breakdown (of the primary vortices) crosses the trailing edge at about the same angle of attack where the secondary vortices apparently disintegrate. This happens at around $10^{\circ}$. This angle can be viewed as analogous to the angle where the lift curve for a classical airfoil first departs from strict linearity. With increasing angle of attack, breakdown rapidly propagates upstream, and the fluid bounded by the leading edge shear layer markedly slows downs. But the leading edge shear layer rollup is still present. The loss of balance between vorticity generation at the leading edges and vorticity 
downstream convection in the LEV cores renders a steady flow situation impossible, whence the left and right LEV breakdown locations are unsteady. However, the leading edge shear layer separation is still relatively coherent; the shear layer still rolls up, and the flow over the leeward side of the wing is still unseparated inboard of this rolled-up structure. When the flow retardation on the left and the right panels of the wing reaches the wing centerline (which occurs at around $20^{\circ}$ angle of attack), the flowfield over the leeward side becomes a "separation bubble" over the entire planform. This is evidently the final stage before the wing acts like a bluff body, at yet higher angles of attack.

\subsection{Recommendations for further work}

Recommendations are divided into three groups: (1) refinements in analysis of the data already taken, (2) extensions of experimental technique to obtain further data with the same models and testing conditions, and (3) extensions to the scope of the experiment itself.

(1) The most obvious recommendation is that a more complete and robust method is necessary for documenting SPIV data from successive downstream crossflow planes. This would allow one to more rigorously track LEV trajectories, to document downstream evolution of unsteadiness in the velocity field, and in general to better perceive the three-dimensional structure of the velocity field. In particular, one could further elucidate how close the flow field is to being conical - by, for example, tracking the downstream growth of peak circulation.

A larger data set of the three-plane SPIV would allow for a similar approach to the vorticity field. With suitable post-processing techniques, one could, for example, 
construct vortex tubes, and thus obtain a better metric for defining the shape and extent of the leading edge vortex, and possibly of the outboard stagnation region as well.

The question of the balance of vorticity produced at the leading edge vs. that convected downstream through the mechanism of LEVs, would be further elucidated by attempting to evaluate the terms in the steady incompressible vorticity equation. This could in principle be done with the "three-plane" data sets where the three components of vorticity are available. However, the role of unsteadiness, not captured by those data, is unclear.

In terms of the stereoscopic PIV technique, more calibration tests and a more rigorous approach to error analysis is necessary. One must carefully distinguish between errors introduced by the PIV algorithm itself vs. those caused by the stereo extension of PIV. Specifically, more work is required to elucidate the role of rotational misalignment between the calibration grid and the interrogation plane.

(2) Of most immediate importance is to extend the SPIV test matrix to higher angles of attack for the $65^{\circ}$ wing. In the present data set, information about the velocity field in post-breakdown conditions was not available, thus limiting the scope of the comparison between the $50^{\circ}$ and $65^{\circ}$ wings. It particular, it would be interesting to observe the condition of the leading edge shear layer when the LEV has already broken down.

Furthermore, SPIV data need to be taken at crossflow planes further downstream, closer to the trailing edge. Such a testing scheme would greatly benefit from automation of the stereo PIV setup and calibration technique, whose present complexity makes an aggressive test matrix intractable. Alternatively, an interesting rearrangement of the PIV 
setup would be to align the laser light sheets parallel to the model planform, located at stations above the wing, on the leeward side. This is especially appropriate for the lower angles of attack, where the salient flow features as viewed in the crossflow planes are small and confined to the vicinity of the wing leeward surface.

Flow visualizations could be enhanced by injecting a dye that fluoresces in a laser light sheet; for example, rhodamine in a Nd:YAG laser light sheet, and retaining the stereo PIV camera setup. This was unsuccessfully attempted in the present study, with difficulties caused by insufficient dye concentration. Dye concentration could then be analyzed quantitatively, beginning with the application of SPIV dewarping and triangulation to images produced by the two cameras.

Long-period fluctuations are not readily captured by the present implementation of DPIV. Recording 30 images/sec is too data-intensive to be used for records on the order of a minute or longer. Rejecting, say, nine out of every ten frames, can allow for sparser data and less computationally intensive, long temporal records.

(3) The most obvious extension to this study is to consider a delta wing of yet smaller sweep than $50^{\circ}$; for example, $45^{\circ}$ or even $40^{\circ}$. Then one could compare the separation from a truly "high aspect ratio" wing with that of the two wings considered above. Of course, at such low sweep angles, classical tip stall becomes an issue, and complicates the investigation. However, the bulk of the PIV experiments were for crossflow planes near the apex, far from the wing tips. It remains an open question as to how "far" the tips and trailing edge have to be from the apex, before one can decidedly conclude that streamwise gradients dominate the crossflow gradients, and not vice versa, 
as for the slender delta wing. Indeed, the meaning of the term "slender delta wing" still lacks definitive resolution.

The issue of Reynolds number effects has not been sufficiently explored. PIV tests for the $50^{\circ}$ wing at the $32 \mathrm{~mm} / \mathrm{s}$ test speed would be instructive in further studying the primary leading edge vortex core velocity distribution, and the possible role of secondary vortices. Likewise, testing at higher Reynolds number is required to justify the conjectures on the Reynolds number dependency of the "stagnation" region outboard of the primary LEV. Unfortunately, such tests would not be handled well by the present experimental setup, e.g., due to vibration and deflection of the mirrors in the test section at higher flow speeds.

Another area of further investigation is that of flow fields of wings rolled to some nonzero angle. The present experimental setup permits such investigations, both for flow visualization and SDPIV, for arbitrary angles of roll and for all angles of attack possible for zero roll. Comparison based on "effective sweep" arguments can be made between a slender wing at high roll angle, and a less slender wing at small or zero roll angle.

As a side note, it would be interesting to investigate the relationship between the flow over the planform (both primary and secondary vortices) and the tip vortices in the near wake. This is a natural choice, especially for the higher aspect ratio wings, which are expected to have a larger contribution to total lift from the potential flow (of which the lifting line is the limiting case). Visualization of tip vortices was attempted but abandoned in this experiment. Again, the problem was one of insufficient dye concentration. Evidently, the core suction in the tip vortices in the near wake (say, less than one root chord length downstream of the trailing edge) was too small to appreciably 
entrain enough dye. But it can be conjectured that especially for those angles of attack where breakdown is downstream of the trailing edge, that complicated mutual rollup of the primary LEVs, secondary LEVs, and the tip vortices have profound consequences on the streamwise convection of vorticity over the wing planform - and thus, affect the stability of organized flow separation over the entire wing, including the near-apex region.

Finally, we note that it is well known that the peak lift coefficient of slender delta wings occurs well after vortex breakdown has crossed the trailing edge - and thus, deep into stall, in the context of the definition of "stall" proposed in 7.4 above. It would be instructive to measure if the peak lift indeed occurs similarly for the $50^{\circ}$ wing, and what consequences that has for other aerodynamic coefficients, chiefly the pitching moment.

And what of the crumbs? Is one to entertain the hope that whereas some have been picked up, many more have fallen? 


\section{Appendix A The Water Tunnel and Sloshing}

The water tunnel facility used for the experiments in this work is shown in Figure 3.1 above. Test section mean operating parameters are mentioned next, with some comments on turbulence intensity. Then, the unexpected presence of unsteadiness is described. Finally, the method used to remove the unsteadiness is outlined.

A.1 Test section flow rate and circuit losses

Flow speed in the test section is shown Figure A.1.

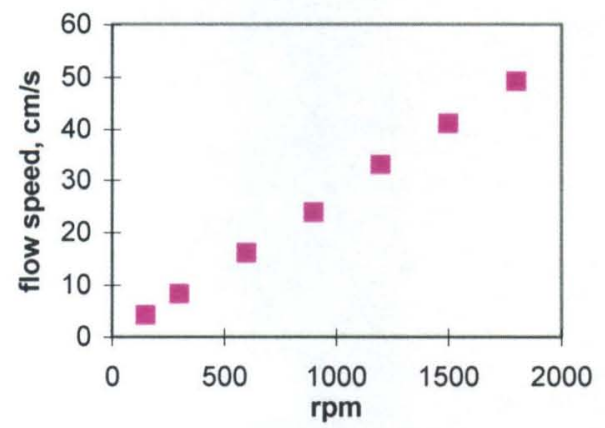

Figure A.1. Test Section Flow Speed vs. Pump RPM

It is insightful to consider the head loss across the circuit; that is, how much of a pressure rise across the pump actuator disk is required to operate the tunnel, as a function of test section flow speed. This is shown in Figure A.2.

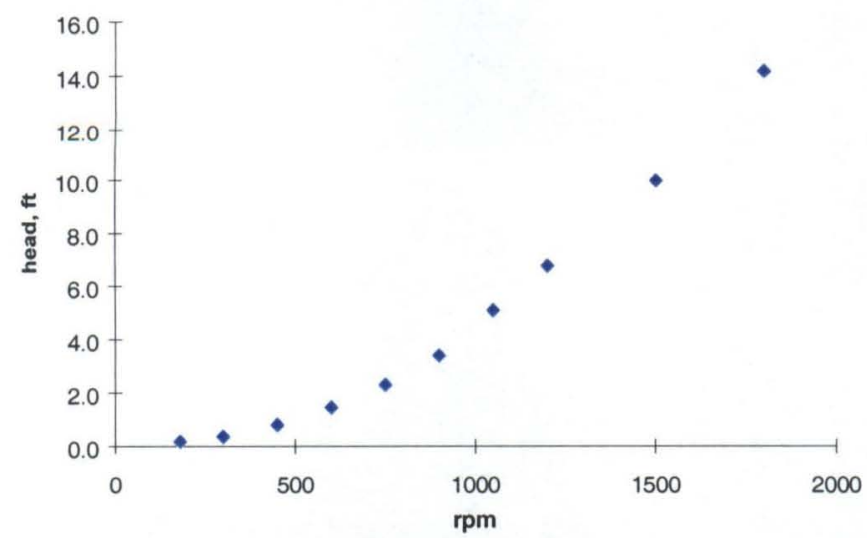

Figure A.2. Circuit Head Loss vs. Pump RPM 
The measurement was taken by comparing data from multiple static pressure ports upstream and downstream of the pump housing. In practice, an initial estimate of such data is used to size the pump and motor to drive tunnel circuit. The curve follows the usual quadratic relationship ${ }^{83}$ of pressure vs. velocity. At the maximum pump speed of $1800 \mathrm{rpm}$, the head loss is about 14 feet of water.

\section{A.2 Some comments on turbulence intensity}

The turbulence intensity in this facility has been quoted at approximately $1.5 \%$. This is somewhat higher than the reported values for most free surface water tunnels of this type. The issue of the extent to which ambient turbulence affects marginally stable structures in the delta wing flow field, or how results may be "contaminated" by turbulent fluctuations, remains an open question. The quoted turbulence level is based on a survey of a limited number of single point measurements of the velocity component in the free stream direction only, conducted with single-component forward scatter LDV. More precisely, "turbulence intensity" is quoted as:

$$
\frac{\left|U_{\text {meas }}^{2}-\bar{U}_{\text {meas }}^{2}\right|^{0.5}}{\bar{U}_{\text {meas }}}
$$

$U_{\text {meas }}$ is the instantaneous measured z-component (in keeping with the notation of this work) of velocity, and the "bar" superscript is the temporal average.

The LDV, in its present implementation, itself has a noise threshold comparable to that of the signal coming from the velocity measurement. 


\section{A.3 Sloshing: time traces of test section flow velocity}

Soon after the tunnel was first run, periodic high amplitude, low frequency

oscillations in the free surface were observed across the entire length of the water tunnel, soon after the tunnel was first operated. LDV measurements of the free stream flow in the test section showed that these oscillations were shallow water waves that resulted in a "sloshing" of up to $8 \%$ peak-to-peak in the flow velocity magnitude. The relationship of free surface elevation and bulk flow velocity is schematically depicted in Figure A.3.

The frequency of the shallow water wave is given by $\mathrm{Kundu}^{84}$, for example.

$$
f=\frac{\sqrt{g H}}{L}
$$

Here $L$ is the nominal total length of the part of the water tunnel exposed to the atmosphere, and $H$ is the water depth, taken in some averaged sense. The sloshing frequency was measured to be $1 / 6 \mathrm{HZ}$, which is in rough correspondence with the $\sim 10 \mathrm{~m}$ tunnel length and $\sim 60 \mathrm{~cm}$ nominal average depth. It was found that various modifications to the tunnel plumbing, such as reducing the number of elbows, were ineffective in reducing sloshing, though they of course affected total circuit head loss and consequently the motor rpm necessary to achieve a particular tunnel flow speed. However, installation of an airtight cover over the inlet plenum and contraction section had a profound effect on dampening the sloshing. During the delta wing experiments, the measured sloshing was reduced to a manageable level. The residual slosh amplitude is comparable to the "turbulence" amplitude as measured by the LDV signal.

The effect of the lid placed over the inlet plenum and contraction section is quite significant. The following shows time traces of flow speeds at various pump speeds. 
Figure A.4 shows the effect of the plenum lid on time traces of the freestream $U$ component of velocity for a range of nominal tunnel speed settings, while Figure A.5 shows comparable results prior to the installation of the plenum lid.

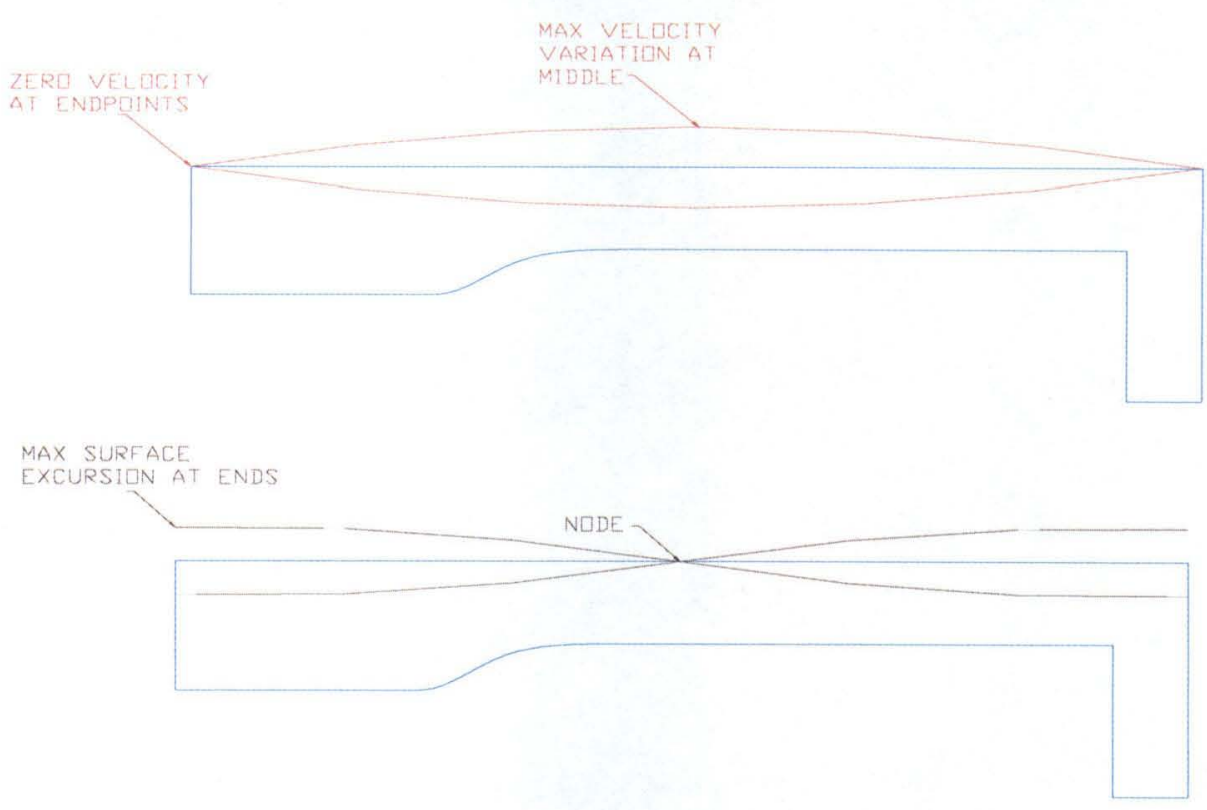

Figure A.3 . Conceptual sketch of free surface "sloshing"

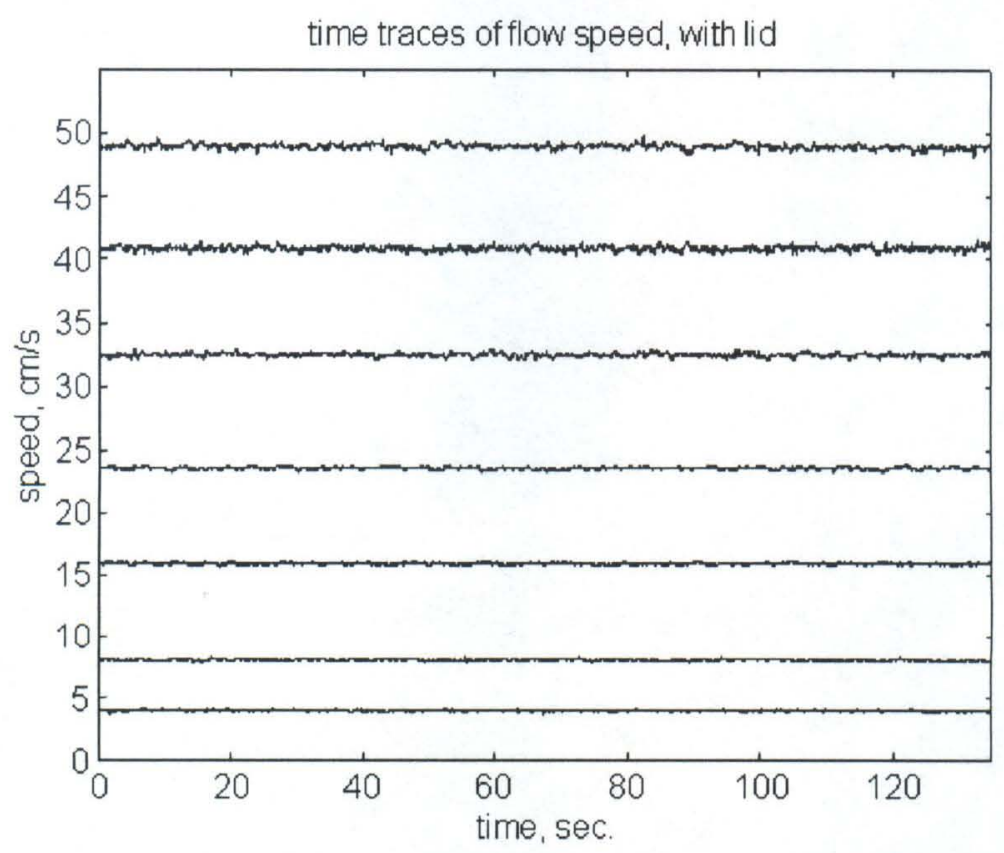

Figure A.4. Time traces of free stream velocities, with sloshing dampened 
It is seen that these data are a significant improvement over those taken prior to the installation of the lid. These are shown below (mean speeds are not necessarily consistent with the above plot):

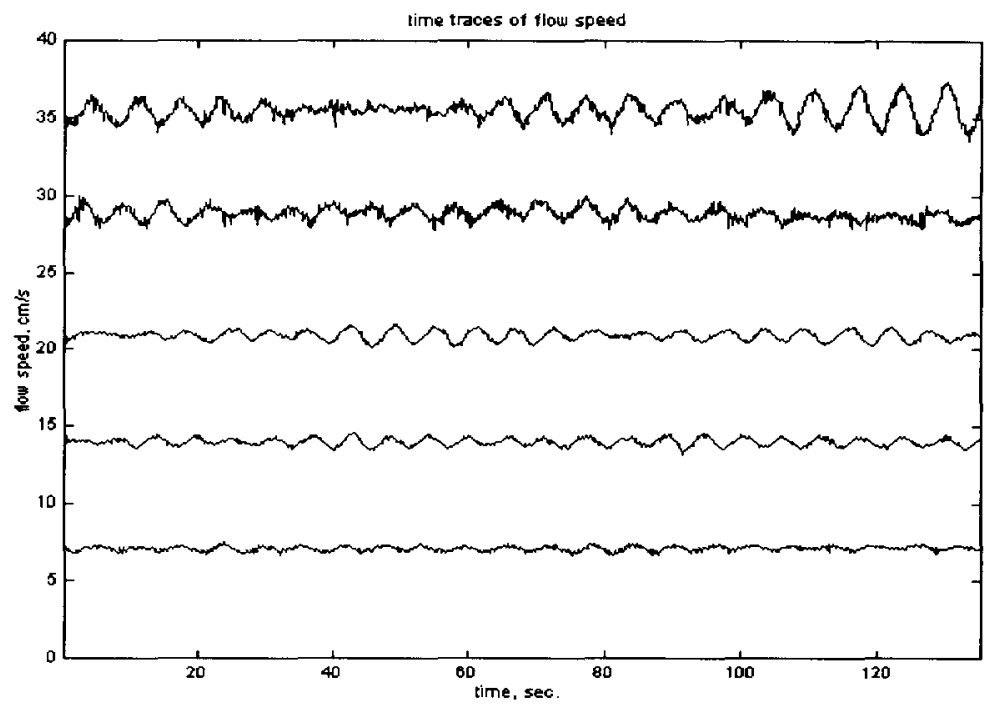

Figure A.5. Time traces of free stream velocity, with sloshing

"Theoretical" motivation for why the lid is so effective can be considered as follows. The area of the hatched region in the top view of the tunnel (Figure 3.1) is approximately $2.4 \mathrm{~m}^{2}$. The maximum amplitude of the free surface oscillation, at maximum tunnel speed, was about $5 \mathrm{~mm}$, yielding a total volume of "displaced water" of about $0.008 \mathrm{~m}^{3}$, assuming a parabolic free surface displacement profile.

This can be compared to the volume of water "displaced" by the sloshing velocity. If we subtract the mean flow speed from its "filtered" time trace, the result resembles a sine wave with a period of about 6 seconds and amplitude of about $4 \%$ of the mean signal; for example, about $1.6 \mathrm{~cm} / \mathrm{s}$ at the maximum tunnel speed. The area under a half-period of this sine wave of period $\mathrm{T}$, multiplied by the tunnel test section crosssectional area $\mathrm{A}$, gives the displaced water volume. 


$$
V o l=A \int_{0}^{T / 2} V(t) d t=A \int_{0}^{3}\left(0.04 V_{0}\right) \sin \left(\frac{2 \pi}{6} t\right) d t=0.08 A V_{0}
$$

The filtered test section flow speed is denoted by $V_{0}$. With $40 \mathrm{~cm} / \mathrm{s}$ flow speed and $0.27 \mathrm{~m}^{2}$ test section cross-sectional area, the resulting volume is $0.0085 \mathrm{~m}^{3}$. This number is comparable to volume displaced by the free surface oscillation in the upstream plenum.

The idea, then, is that by suppressing the oscillation of the free surface in the upstream plenum and contraction section (these are the largest areas of the tunnel open to the atmosphere), the water effectively displaced by the sloshing, and hence the wave energy, has nowhere to go. This can be accomplished by placing an airtight cover over the intake plenum and contraction section. The cover is essentially a box which terminates on its downstream end with a semi-flexible Plexiglas lip which penetrates the free surface. Ideally, the lip would terminate just beneath the free surface, to minimize effects on the velocity profile in the test section. 


\section{Appendix B SPIV Validations and Correction of Parallax Distortion}

Perhaps the most basic validations of stereo digital particle image velocimetry in a water tunnel are those that attempt to isolate the basic difficulties of PIV in general from those peculiar to SPIV. The former include, for example, lighting and contrast issues, particle size, particle concentration and frame-to-frame particle correlations. As such, an artificial target of particle speckling was used to simulate displacements. This yielded information regarding the parallax error alluded to in the text. Parallax error is discussed further below, with a suggested a posteriori method of mitigating the effects of this error.

\section{B.1 Orthogonal translations of an artificial particle grid}

A nominally random speckling of "particles" (white dots on a black background) printed onto paper and sandwiched between two Plexiglas plates was mounted on an $x-y-$ $\mathrm{z}$ traversing mechanism and illuminated with white light from underneath the tunnel test section. The speckling faced normal to the test section axis, and was thus aligned with where the laser light sheet would have been. A white-light source placed underneath the test section illuminated the particle speckling. This setup avoids the use of the laser light sheet, and therefore, issues of alignment with respect to the light sheet. The Plexiglas plate facing the cameras was kept as thin as possible (1/8") to avoid refraction problems caused by light passage through the plate. This is similar to the issue of multiple indices of refraction discussed in Chapter 2.

By translating this particle speckling in the three axes of motion, various "velocity" directions can be simulated. It would also be instructive to rotate the 
speckling in various directions, but such experiments were not pursued in the present study.

\section{B.1.1 Pure out of plane motion}

In this case, the particle speckling is moved along the free stream flow direction in the test section, thus simulating a free stream flow. The input motion is therefore purely out-of-plane. The motion as registered by SPIV is shown in a 3-D view in Figure B.6. In these tests, $35 \mathrm{~mm}$ focal length lenses were used. The axis units used in this section are intermediate quantities in the SPIV image processing, and are unimportant for purposes of the present discussion.

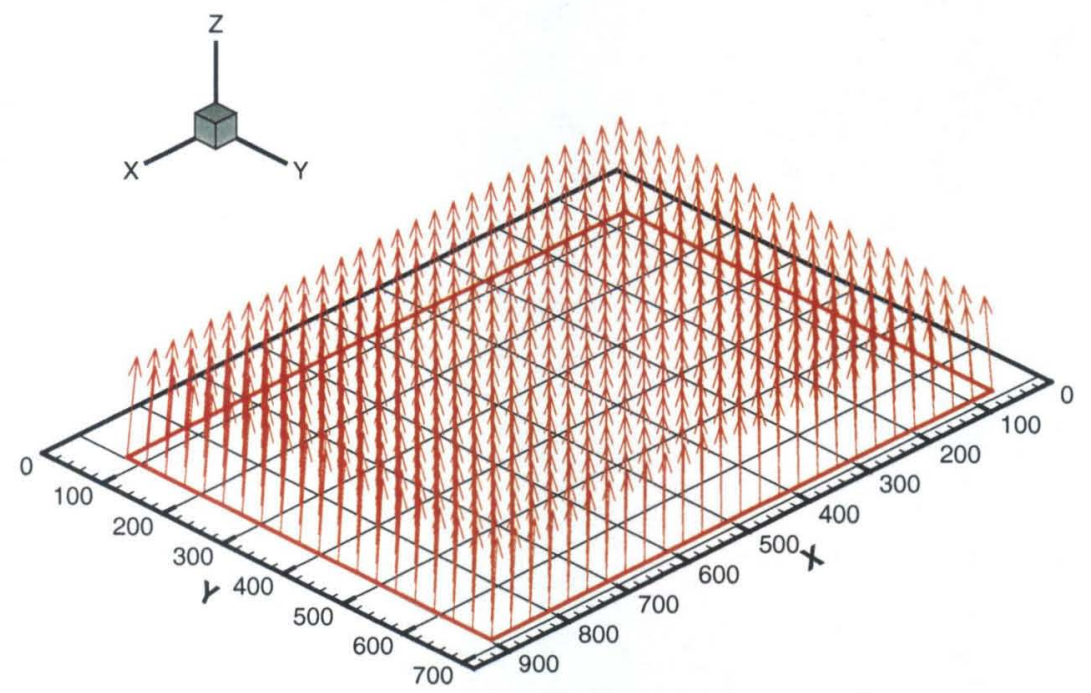

Figure B.6. Pure out-of-plane artificial displacement

Discrepancy between real and measured displacement can be considered separately in the in-plane and the out-of-plane directions. Out-of-plane error - that is, the amount by which SPIV underestimates or overestimates the actual input displacement is uniform over the field of view, and is approximately $4 \%$. The in-plane error, a strictly spurious quantity introduced by the SPIV procedure, is essentially zero at the image 
center, and increases radially in going away from the center. This error is already evident in Figure B.6, but is seen more clearly by considering the planar projection of the velocity vectors; that is, from will be referred to as the "streamwise" view (Figure B.7). Approximately $10 \%$ maximum in-plane error is found in the present case. As can be seen in Figure B.7, the x-direction error is substantially greater than the y-direction error - in this case, by about a factor of 3 .

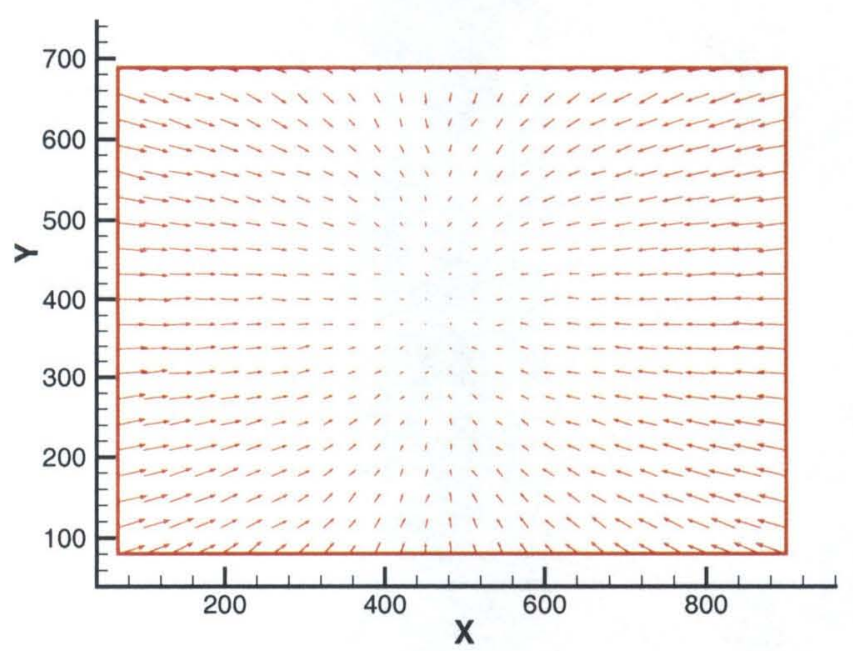

Figure B.7. Planar projection of out-of-plane motion

Figure B.7 shows what might be regarded as a parallax error. This is discussed further below.

\section{B.1.2 In-plane translations}

Here the artificial particle speckling is translated in its own plane - first, along the x-axis. A 3-D view of the resulting motion is shown in Figure B.8. 


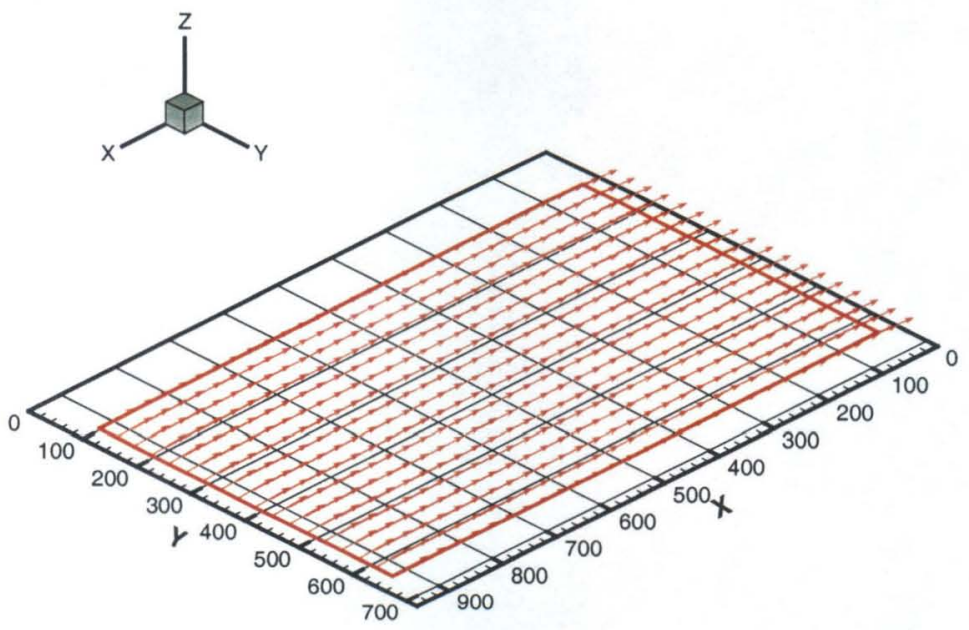

Figure B.8. Left-right planar translation of artificial particle speckling

Once again the in-plane and out-of-plane errors can be considered separately. In-plane, the discrepancy between input and recorded displacement is approximately $2 \%$. To view the out-of plane error, the out-of-plane coordinate $(Z)$ can be expanded by a factor of 100 , thereby exaggerating out-of-plane error. The result is shown in Figure B.9. This has the appearance of a rotation, with peak error also of approximately $2 \%$.

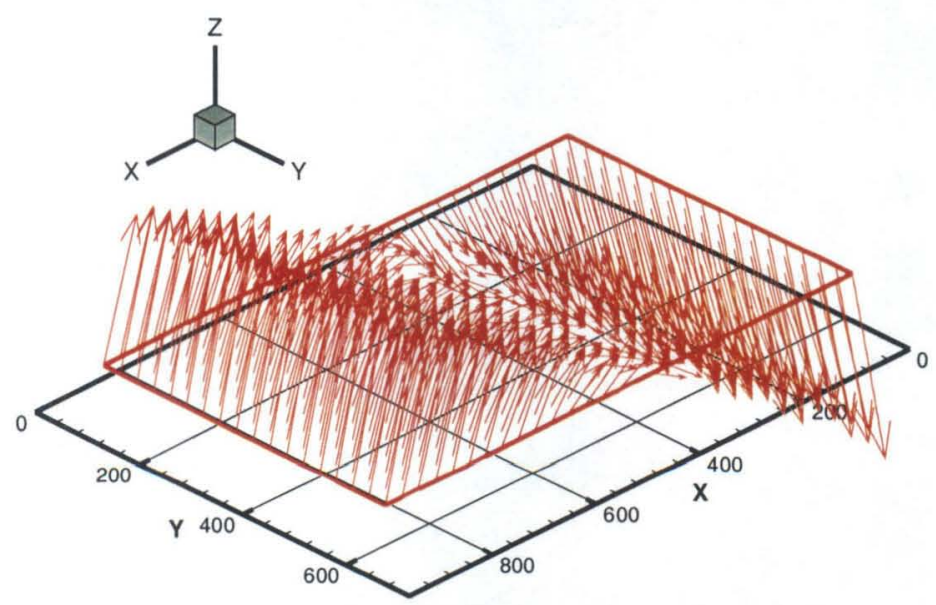

Figure B.9. Left-right translation, with 100x magnification of Z-axis

In-plane vertical (i.e., along y-axis) translation (Figure B.10) produces approximately $1 \%$ error, without an identifiable shape, such as that of Figure B.9. 


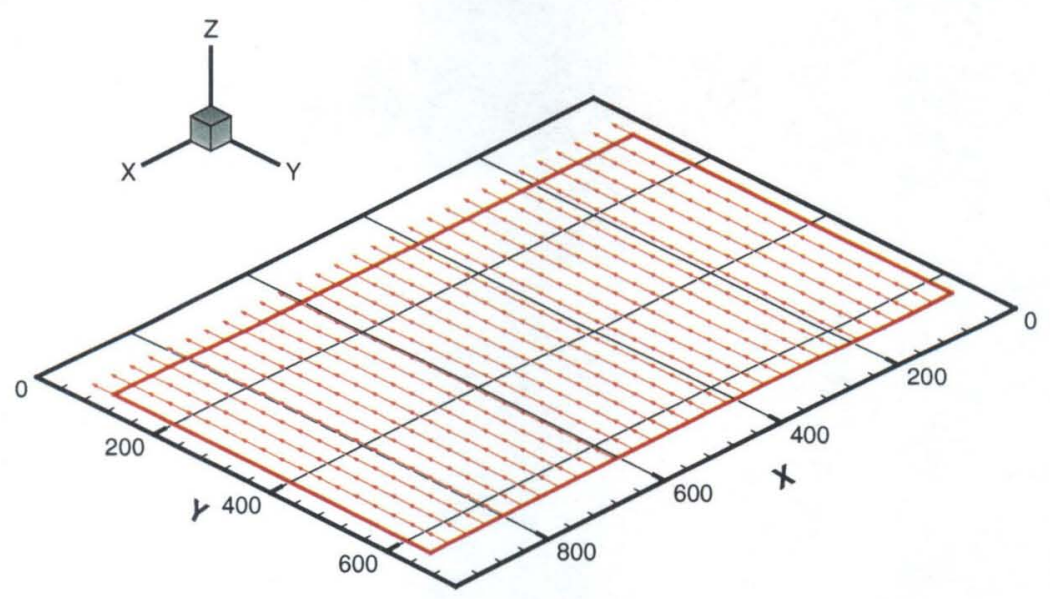

Figure B.10. In-plane vertical translation

From these calibration tests, it is apparent that the present implementation of SPIV introduces a $1-4 \%$ error in the measurement of a particular displacement, and a much higher parallax error.

\section{B.2 Further comments on parallax distortion}

Parallax distortion is manifested in the presence of apparent in-plane velocity, measured for flows with purely out-of plane physical velocity, where the former can be as high as $\sim 10 \%$ of the latter in magnitude, when using a wide-angle photographic lens. The actual amount of error depends strongly on the focal length of the SPIV camera lenses; shorter focal lengths produce greater errors. Curiously, this result is not only not corrected in the rotational stereo technique and its triangulation procedure, but is in fact noticeably worse than the parallax error for the simple on-axis 2-D PIV, where a single camera is facing normal to a pure out-of-plane displacement field. One can surmise that this result is not an inherent indictment of the SPIV technique in general, but weakness in some part of its present implementation. Fortunately, the spurious in-plane velocity field is irrotational, and thus does not contaminate the vorticity-based calculations. 
As an a posteriori effort to account for such an error, an additive "correction" is proposed. The technique makes three main assumptions:

- There is a "center point" in the image, where error is negligibly small. This center need not be the geometric center of the image.

- The magnitude of the distortion varies linearly with respect the magnitude of the measured out-of-plane velocity; that is, the ratio of in-plane error to out-of plane velocity is a constant, at any given point in the image, regardless of how the out-ofplane velocity varies.

- The variation of the distortion varies linearly, in going radially outward from the center point. In fact, it is assumed that when the interrogation window array is described in polar coordinates, there is no azimuthal dependency. However, the xdirection of the variation in general differs from the y-direction.

A typical case for $\mathrm{x}$-direction error variation for tunnel free stream data is shown in Figure B.11. The independent variable is CCD pixel units. It suggests that the linearity part of the assumption is well justified.

Y-averaged in-plane bias as fcn of $x$

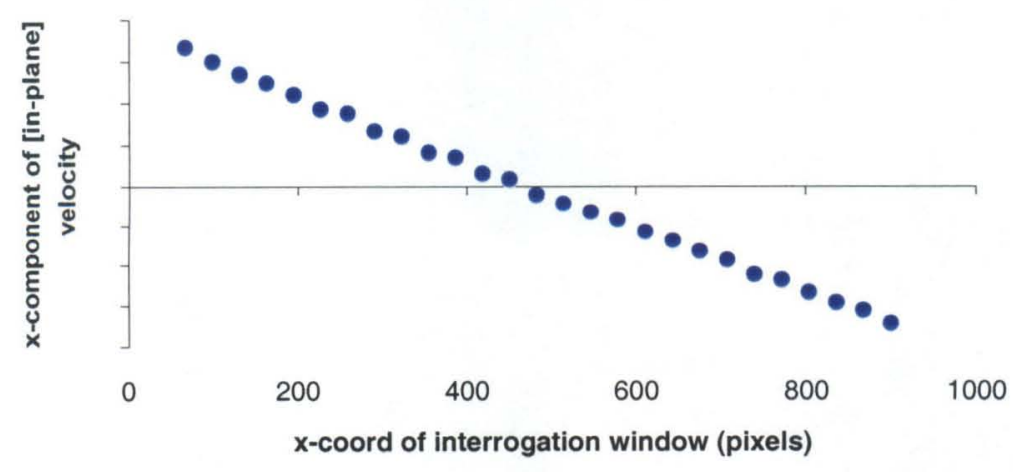

Figure B.11. Behavior of parallax error in x-direction 
In addition, it is that assumed in a "real" data file, with all three velocity components present in the flow, contamination of the in-plane data by parallax error does not in turn affect the out-of-plane measurement; whence it is conjectured that the perspective correction itself should only apply to the in-plane components, and not, in an iterative sense, the out-of-plane component as well.

\section{B.3 Error calculation}

A smoothed 3-component file of nominally purely out-of-plane motion, obtained either from water tunnel free stream data (flow normal to the light sheet), or from translations of an artificial particle target, is used as a "baseline" velocimetry case. The image needs to be smooth to avoid introducing small-scale random error. The average out-of-plane velocity component for the entire image is calculated first. Then the inplane velocity magnitude, $\sqrt{u^{2}(x, y)+v(x, y)}$, is computed for each cell.

The location of the "zero distortion center" is found by linear regression in the $\mathrm{X}$ and $\mathrm{Y}$ - directions independently, with sub-pixel fitting.

\section{B.4 Error correction}

The above results are applied on a window-by-window basis to subtract the elliptic cone of error from the in-plane components of the given velocity field. For each interrogation window, the in-plane velocity components are corrected as follows:

$$
\begin{gathered}
u_{\text {corr }}\left(x_{i}, y_{j}\right)=u_{\text {meas }}\left(x_{i}, y_{j}\right)-R m_{x} \cos \theta \\
v_{\text {corr }}\left(x_{i}, y_{j}\right)=v_{\text {meas }}\left(x_{i}, y_{j}\right)-R m_{y} \sin \theta
\end{gathered}
$$


Where $R=\sqrt{\left(x_{i}-X\right)^{2}+\left(y_{j}-Y\right)^{2}}$ is the radial distance from the zero-error center to the window, $\left(x_{i}, y_{j}\right) ; \theta=\arctan \left(\frac{y_{j}-Y}{x_{i}-X}\right)$ is the azimuthal angle from the zero-error center to the window; and $m_{x}, m_{y}$ are the slopes of the error distribution.

Applying this formulation to the above-mentioned test file, one obtains output with nearly zero in-plane velocity.

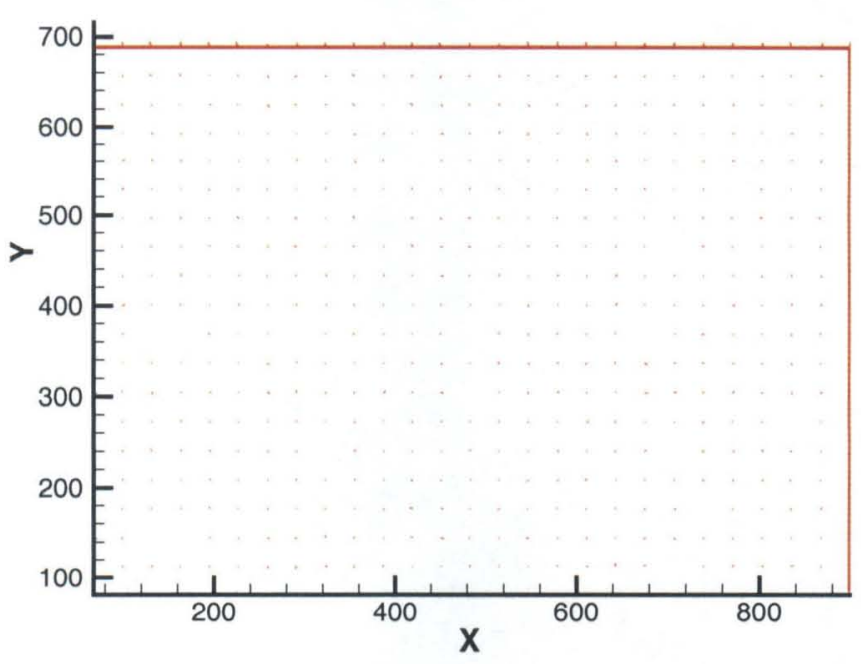

Figure B.12. Corrected parallax error

The peak normalized residual error magnitude is now $0.8 \%$. The above manipulations were performed on the same data file as the one used to construct the error correction; that is, the assumptions of error linearity with respect to coordinate location in the image were verified, but not the assumption of linearity with magnitude of out-ofplane measured displacement. By considering a data file from measurements with the same optical settings but with different out-of-plane displacement, the latter assumption can be verified as well. This is considered in the following two images, the "before" and "after." The measured out-of-plane velocity is nominally double that of the previous figures. 


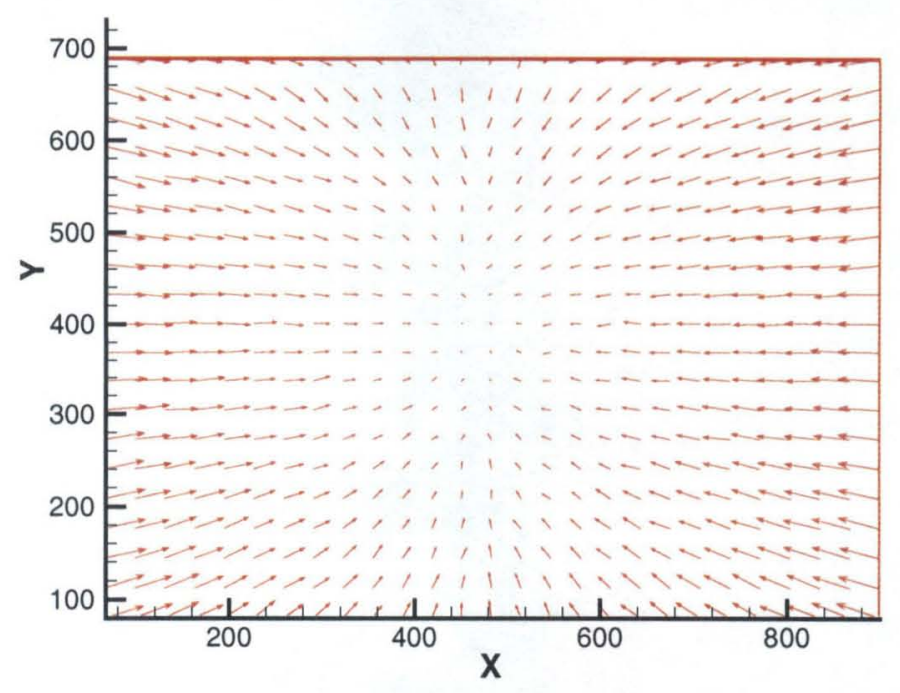

Figure B.13. A second test case for parallax correction

The raw image shows in-plane error roughly double that of the first raw image.

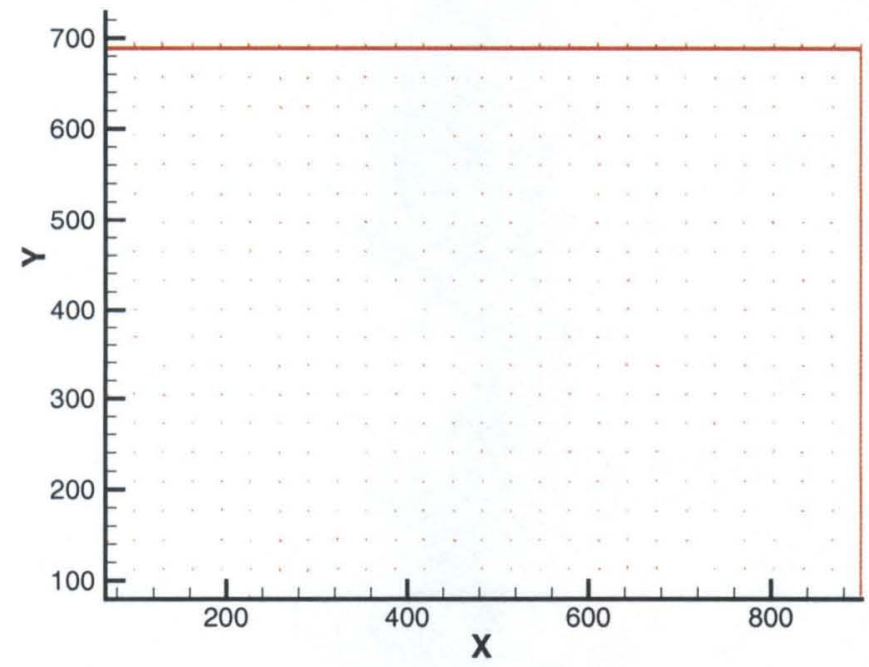

Figure B.14. Correction of parallax error in second test case

The corrected file is qualitatively far cleaner. Quantitatively, the normalized inplane error again peaks at about $0.8 \%$. Thus, we have good supporting evidence for all three of the original assumptions on which the in-plane error correction method is based. 


\section{Appendix C Mirror-Related SPIV Geometric Calculations}

The text (Chapter 2) describes the theory of rotational stereoscopic PIV. Here, we consider some technical aspects of its implementation in a water tunnel.

\section{C.1 Triangulation parameters for standard camera arrangement}

First, the simplified case where no mirrors are present (Figure C.15) is considered. The "camera focal point" is the location of the single element lens "equivalent" to the multi-element photographic lens; thus, for a Nikkor 105mm macro lens, the equivalent single element lens is, say, a simple bispherical lens, located some distance in front of the ccd plane. That distance is given by applying the lens formula to this equivalent lens, the location of the ccd, and the location of the object being viewed:

$$
\frac{1}{f}=\frac{1}{a}+\frac{1}{b}
$$

where $\mathrm{f}$ is the lens focal length, $\mathrm{a}$ is the distance from the lens to the $\mathrm{ccd}$, and $\mathrm{b}$ is the distance from the lens to the object. Since $f$ and the sum $[a+b]$ are known, we can solve for $b$ - which locates the "camera focal point."

We refer to a coordinate system aligned with the test section centerline. In the figure, positive $\mathrm{X}$-coord is up, positive $\mathrm{Z}$-coord is to the right, and positive $\mathrm{Y}$-coord is out of the page, with coordinate origin as shown.

Seven quantities are needed: downstream distance from interrogation plane to left and right camera axes, $z_{C L}$ and $z_{C R}$, respectively; distance of left (right) camera focal point from left (right) tunnel wall exterior, $x_{C L}\left(x_{C R}\right)$; distance of interrogation region centerline from tunnel centerline, $x_{i}$; test section half-width, $T$; and test section wall thickness, $W$. With these measurements, together with information about the size of the 
viewing area (available from counting grid markings in the calibration images), the viewing geometry is completely specified (Figure C.15).

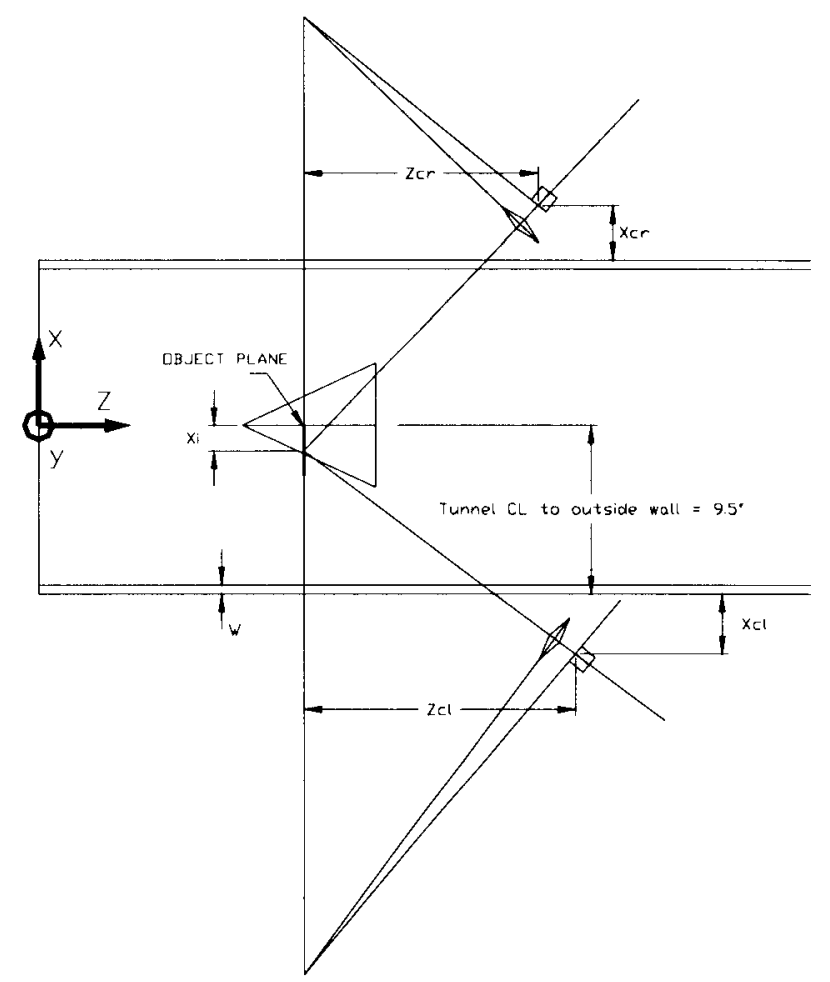

Figure C.15. Equivalent "standard" SPIV arrangement

Next, consider a pixel in the interrogation region. Let it have model-frame coordinates $(x, y)$; that is, relative to the center point of the interrogation region. Then the viewing angles of each pixel, as functions of individual PIV interrogation window locations in the image plane, are found from the simple relations

$$
\begin{gathered}
\alpha_{L}(x, y)=\arctan \frac{x_{C L}+W+T+x_{i}-x}{z_{C L}} \\
\alpha_{R}(x, y)=\arctan \frac{x_{C R}+W+T-x_{i}-x}{z_{C R}} \\
\beta_{L}(x, y)=\arctan \frac{y}{z_{C L}}
\end{gathered}
$$




$$
\beta_{L}(x, y)=\arctan \frac{y}{z_{C R}}
$$

Note that the alphas are always greater than zero, but the betas are sometimes positive, sometimes negative, and zero at the image center, since the cameras are in the same vertical plane as the interrogation region centerline for this particular arrangement.

\section{C.2 Triangulation parameters for arrangement with mirrors}

Next, we introduce the mirrors. The placement and orientation of the mirrors is dictated by the fact that the mirror face must lie along the bisector of the angle formed between the camera focal point in the unfolded setup, the focal point in the folded setup, and the mirror centerline. All of this is subject to the constraint that the mirrors be placed as close as possible to the tunnel walls, for purposes of reducing interference inside the test section, and to bring the mirrors as close as possible to the photographic lenses. On the other hand, the mirrors should be as far away from the model as possible, to reduce upstream disturbances. But if the mirrors are too far downstream, the effective camerato-camera opening angle becomes small, and accuracy of the measurement of the zcomponent of velocity suffers ${ }^{85}$. Figure C.16 illustrates the geometry.

The rays locating the lens and camera according to the Scheimpflug criterion are now oriented at an unnatural angle, but the optical effect is the same (apart from the reversal of the images caused by the mirrors). 


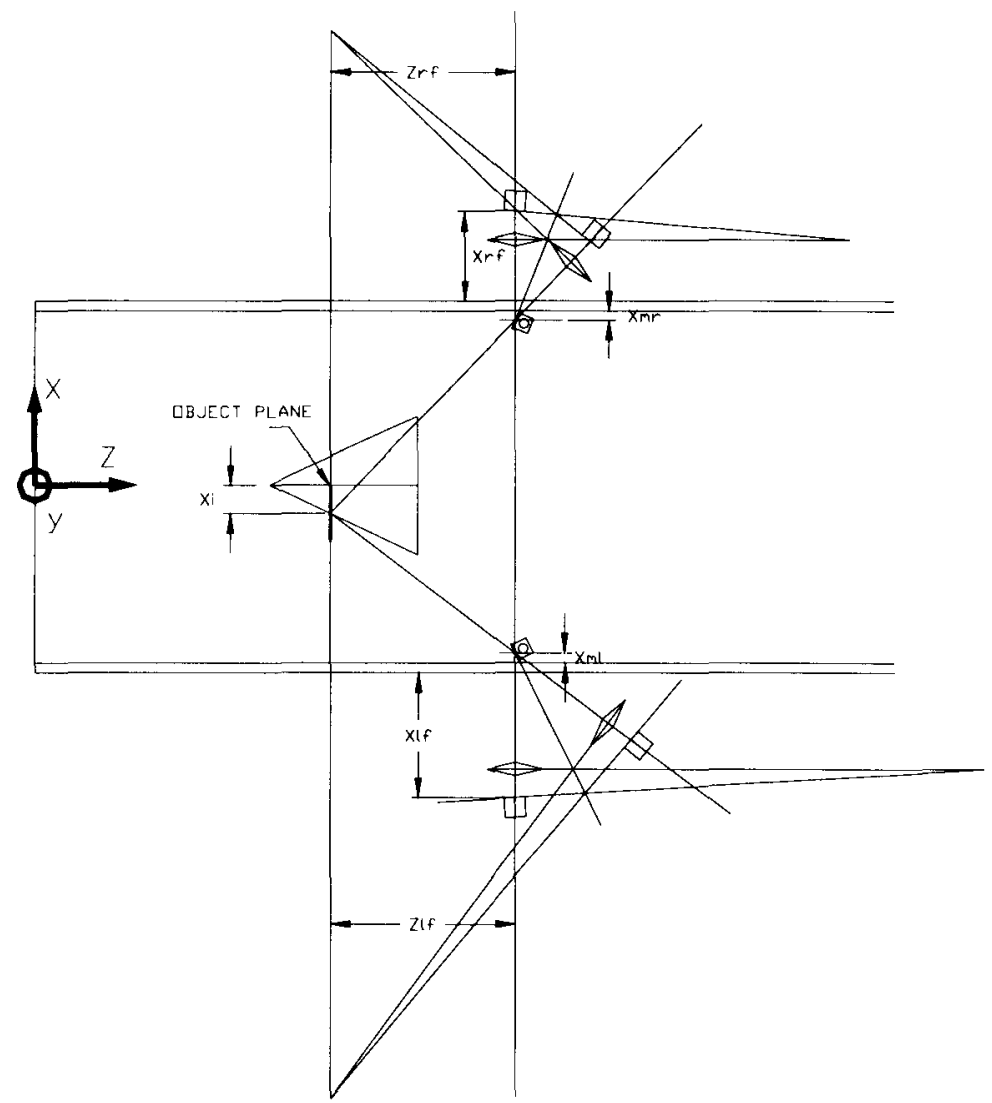

Figure C.16. "Unfolding" of camera lines of sight

The situation is better viewed by magnifying the region around one mirror - say, the left.

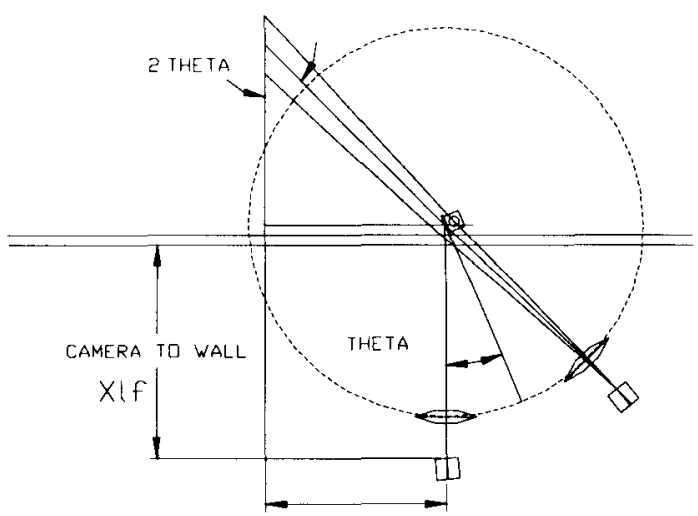

INTERRLGATIIN PLANE TL CAMERA AXIS $Z 1 f$

Figure C.17. Magnified view of left camera line of sight 
New measurements describe the camera locations:

- distance of (folded) left camera to outside of left tunnel wall, $x_{L F}$

- distance of (folded) right camera to outside of right tunnel wall, $x_{R F}$

- downstream distance of (folded) left camera axis to subject plane, $z_{L F}$

- downstream distance of (folded) right camera axis to subject plane, $z_{R F}$

- distance from left mirror centerline to right tunnel inner wall, $x_{M R}$

- distance from right mirror centerline to left tunnel inner wall, $x_{M L}$

The angle "theta" is just half the viewing angle to the center of the subject; there is a left and a right theta, $\theta_{L}$ and $\theta_{R}$, respectively. Of course, the $\theta_{L}$ and $\theta_{R}$ also happen to be the mirror orientation angles, and are given by:

$$
\begin{gathered}
2 \theta_{L}=\arctan \frac{z_{L F}}{T-x_{i}-x_{M L}} \\
2 \theta_{R}=\arctan \frac{z_{R F}}{T+x_{i}-x_{M R}}
\end{gathered}
$$

Finally, the unfolded camera to wall distances, $x_{C L}$ and $x_{C R}$, and unfolded subject plane to camera distances, $z_{C L}$ and $z_{C R}$, can be computed from the following:

$$
\begin{gathered}
x_{C L}=T+W-x_{i}+\left(x_{L F}+W+x_{M L}\right) \cos \left(2 \theta_{L}\right) \\
x_{C R}=T+W+x_{i}+\left(x_{R F}+W+x_{M R}\right) \cos \left(2 \theta_{R}\right) \\
z_{C L}=\left(x_{L F}+W+x_{M L}\right) \sin \left(2 \theta_{L}\right) \\
z_{C R}=\left(x_{R F}+W+x_{M R}\right) \sin \left(2 \theta_{R}\right)
\end{gathered}
$$




\section{References}

${ }^{1}$ Lippisch, A. The Delta Wing. Iowa State U. Press, Ames, 1991.

2 Jones, R.T. "Wing Plan form for High Speed Flight." NACA Report No. 863, 1947.

3 Jones, R.T. "Properties of Low Aspect Ratio Pointed Wings at Speeds below and above the Speed of Sound." NACA Report No. 835, 1946.

${ }^{4}$ Stanbrook, A. and Squire, L.C. "Possible Types of Flow at Swept Leading Edges." Aero. Quarterly, Feb. 1964, pp. 72-82.

${ }^{5}$ Herbst, W.M. "Future Fighter Technologies." J. Aircraft, Vol. 17, No. 8. August 1980.

${ }^{6}$ Polhamus, E. C. Paper \#1 in NASA CP-2416.

${ }^{7}$ Parker, A. G. "Aerodynamic Characteristics of Slender Wings with Sharp Leading Edges - A Review." J. Aircraft. Vol. 13, No. 3, March 1976, pp. 162-168.

${ }^{8}$ Kuechemann, D. The Aerodynamic Design of Aircraft. 1976.

9 Kegelman, J.T. and Roos, F.W. "Effects of Leading Edge Shape and Vortex Burst on the Flowfield of a 70-Degree-Sweep Delta Wing." AIAA paper \# 89-0086, January 1989.

${ }^{10}$ Hoerner, S. Fluid Dynamic Lift. Chap. 17,18. Published by the author, 1976.

1 Verhaagen, N.G. "An Experimental Investigation of the Vortex Flow over Delta and Double-Delta Wings at Low Speed." AGARD CP-342, 1983, pp. 7-1 - 7-16.

12 Rom, J. High Angle of Attack Aerodynamics. Springer, 1989.

13 Sarpkaya, T. "On Stationary and Traveling Vortex Breakdowns" JFM. Vol. 45, part 3. 1971, pp. 545-559.

14 Visbal, M.R. "Computational and Physical Aspects of Vortex Breakdown." AIAA paper \# 95-0585, January 1995.

15 Towfighi, J. and Rockwell, D. "Instantaneous Structure of Vortex Breakdown on a Delta Wing via Particle Image Velocimetry." AIAA Journal. Vol. 31, No. 6. 1993, pp. 1160-1162.

${ }^{16}$ Jobe, C.E. "Vortex Breakdown Location over $65^{\circ}$ Delta Wings - Empiricism and Experiment." AIAA paper 98-2526, June 1998. 
${ }^{17}$ Delery, J.M. and Molton, P. "Topology of the Flow Resulting from Vortex Breakdown over a Delta Wing at Subsonic Speed." Acta Mech. [Supplement] 4: pp. 297304, 1994.

18 Wentz, W.H. and Kohlman, D.L. "Vortex Breakdown on Slender Sharp-Edged Wings." J. Aircraft, Vol. 8, No. 3, March 1971, pp. 156-161.

19 Earnshaw, P.B. and Lawford, J.A. "Low-Speed Wind Tunnel Experiments on a Series of Sharp-Edged Delta Wings." Reports and Memoranda No. 3424, March 1964.

${ }^{20}$ Jenkins, J.E. and Hanff, E.S. "Highlights of the IAR/WL Delta Wing Program." AIAA AFM Conference, 1995; Workshop III - "Delta Wings: Unsteady Aerodynamics and Modeling." August, 1995.

${ }^{21}$ Hemsch, M.J. and Luckring, J.M. "Connection Between Leading-Edge Sweep, Vortex Lift and Vortex Strength for Delta Wings." J. Aircraft, Vol. 27, No. 5. 1990, pp. 473-475.

${ }^{22} \mathrm{Su}, \mathrm{W}$., Liu, M. and Liu, Z. "Topological Structures of Separated Flows about a Series of Sharp-Edged Delta Wings at Angles of Attack up to $90^{\circ}$," in Topological Fluid Mechanics: Proceedings of the IUTAM Symposium. H.K. Moffatt and A. Tsinober (eds.). August, 1989, pp. 395-407.

${ }^{23}$ Freymuth, P. "Vortex Topology of Rectangular Wings in Pictures, Sketches and Conjectures." J. Flow Visualization and Image Proc., Vol. 1, pp. 1-13, 1993.

${ }^{24}$ Roos, F. W. and Kegelman, J. T. "Recent Explorations of Leading-Edge-Vortex Flowfields." NASA High Angle of Attack Technology Conference, Proceedings, 1990; NASA CP 3149, Part 1, pp. 157-172.

${ }^{25}$ Ayoub, A. and McLachlan, B.G. "Slender Delta Wing at High Angles of Attack - a Flow Visualization Study." AIAA paper \# 87-1230, June 1987.

${ }^{26}$ Hall, M.G. "A Theory for the Core of a Leading-Edge Vortex." JFM, Vol. 11. 1961, pp. 209-228.

${ }^{27}$ Earnshaw, P.B. "An Experimental Investigation of the Structure of a Leading Edge Vortex." Aero. Res. Council, London. Rept. No. 22,876, 1961.

${ }^{28}$ Moore, D. W. and D. I. Pullin. "Inviscid Separated Flow over a Non-Slender Delta Wing," JFM, Vol. 305, 1995, pp. 307-345.

${ }^{29}$ Stahl, W.H. "Aerodynamics of Low Aspect Ratio Wings." pp. 3-1 - 3-64.

${ }^{30}$ Polhamus, E. C. "Predictions of Vortex-Lift Characteristics by a Leading-Edge Suction Analogy," J. Aircraft. Vol. 8, No. 4, April 1971, pp. 193-199. 
31 Polhamus, E.C., "A Concept of the Vortex Lift of Sharp Edge Delta Wings Based on a Leading-Edge Suction Analogy.” NASA TN D-3736, Dec. 1966.

32 Kuethe, A.M. and Chow. Foundations of Aerodynamics. Wiley, 1986.

33 Gursul, I. "Criteria for Location of Vortex Breakdown Over Delta Wings." Aero. J. of the Royal Aero. Soc., May, 1995.

34 Wedemeyer, E. H. "Stable and Unstable Vortex Separation." AGARD CP-247, paper \#13. 1979, pp. 13-1 - 13-10.

${ }^{35}$ Ludwieg, H. "Stabilitaet der Stroemung in einem zylindrischen Ringraum." Z. Flugwiss., Vol. 8, 1960, pp. 135-140.

36 Cipolla, K.M. Structure of the Flow Past a Delta Wing with Variations in Roll Angle. Dissertation, Lehigh U., 1996.

37 Lang, N. and Limberg, W. "Construction of Three-Dimensional Flow Structure out of Two-Dimensional Steady Flow Field Velocity Measurements." Exp. in Fluids, Vol. 27, pp. 351-358, 1999.

38 Shih, C. and Ding, Z. "Unsteady Structure of Leading-Edge Vortex Flow over a Delta Wing." AIAA paper \# 96-0664, January 1996.

39 Romano, G.P. "PIV and LDA Measurements Near Walls and in the Wake of a Delta Wing." Optics and Lasers in Engineering. Vol. 16, 1992, pp. 293-309.

40 Shah, P.N., Atsavapranee, P., Hsu, T.Y., Wei, T. and McHugh, J. "Turbulent Transport in the Core of a Trailing Half-Delta-Wing Vortex." JFM (1999), Vol. 387, pp. 151-175.

41 Spedding, G.R., Maxworthy, T. and Rignot, E. "Unsteady Vortex Flows over Delta Wings." pp. 283-287.

${ }^{42}$ Traub, L. W., Rediniotis, O.K., Klute, S.M. and Telionis, D.P. "Instabilities of Vortex Breakdown; Their Structure and Growth." AIAA paper \# 95-2308, June 1995.

43 Lambourne, N.C. and Pusey, P.S. "Some Visual Observations of the Effects of Sweep on the Low-Speed Flow over a Sharp-Edged Plate at Incidence." $R . \& M$. No. 3106 (19,821), A.R.C. Tech. Report. 1959.

44 Honken, A. and Andreopoulos, J. "Instantaneous Three-Dimensional Vorticity Measurements in a Vortical Flow over a Delta Wing." AIAA paper 95-0587, 1995. 
${ }^{45}$ Miau, J.J., Kuo, K.T., Liu, W.H., Hsieh, S.J., Chou, J.H. and Lin, C.K. "Flow Developments above 50-Deg Sweep Delta Wings with Different Leading-Edge Profiles." J. Aircraft, Vol. 32, No. 4, Jul.-Aug. 1995.

46 Zohar, Y. and Er-El, J. "Influence of Aspect Ratio on the Aerodynamics of the Delta Wing at High Angle of Attack." J. Aircraft. Vol. 25, No. 3, March 1988.

47 Bird, J.D. "Tuft-Grid Surveys at Low Speeds for Delta Wings." NASA TN D-5045, 1969.

48 Lee, M. and Ho, C.-M., "Lift Force of Delta Wings." ASME; Appl. Mech. Rev. Vol 43, No 9, September 1990, pp. 209-221.

49 Payne, F. M., Ng, T.T. and Nelson, R.C. "Experimental Study of the Velocity Field on a Delta Wing." AIAA paper A87-1231, 1987.

${ }^{50}$ Raffel, M., Gharib, M., Ronneberger, O. and Kompenhans, J. "Feasibility Study of Three-Dimensional PIV by Correlating Images of Particles Within Parallel Light Sheet Planes. Exp. In Fluids, 1995.

${ }^{51}$ Prasad, A.K. "Stereoscopic Particle Image Velocimetry." Exp. in Fluids, Vol. 29, 2000, pp. 103-116.

52 Soloff, S.M., Adrian, R.J. and Liu, Z-C. "Distortion Compensation for Generalized Stereoscopic Particle Image Velocimetry." Meas. Sci. Technol., Vol. 8, 1997, pp. 14411454.

${ }^{53}$ Willert, C.E. "Stereoscopic Digital Particle Image Velocimetry for Application in Wind Tunnel Flows." Meas. Sci. Technol., Vol. 8, 1997, pp. 1465-1479.

${ }^{54}$ Prasad, A.K. and Jensen, K. "Scheimpflug Stereocamera for Particle Image Velocimetry in Liquid Flows." Applied Optics, Vol. 34, No. 30, Oct. 1995.

55 Prasad, A.K. and Adrian, R.J. "Stereoscopic Particle Image Velocimetry Applied to Liquid Flows." Exp. in Fluids, Vol. 15, 1993, pp. 49-60.

${ }^{56}$ Willert, C. E. Ph.D. Thesis, UCSD, 1992.

57 Erickson, G.E. "Water Tunnel Flow Visualization: Insight into Complex ThreeDimensional Flow Fields." AIAA paper 79-1530, July 1979.

58 Erickson, G.E. "Water Tunnel Studies of Leading-Edge Vortices." J. Aircraft, Vol. 19, No. 6, pp. 442-448. 1981.

59 Poisson-Quinton, P. and Werle, H. "Water-Tunnel Visualization of Vortex Flow." Aeronautics and Astronautics, June 1967, pp. 64-66. 
${ }^{60}$ Thompson, D.H. "A Water Tunnel Study of Vortex Breakdown over Delta Wings with Highly Swept Leading Edges." Aerodynamics Note 356, A.R.L. (Australia), May 1975.

61 Park, H. Ph.D. Thesis, Caltech, 1998.

${ }^{62}$ Westerweel, J., Dabiri, D. and Gharib, M. "The Effect of Discrete Window Offset on the Accuracy of Cross-Correlation Analysis of Digital PIV Recordings." Exp. in Fluids, Vol. 23, 1997, pp. 20-28.

63 Jeon, D. Ph.D. Thesis, Caltech, 2000.

${ }^{64}$ Raffel, M., Willert, C.E. and Kompenhans, J. Particle Image Velocimetry: A Practical Guide. Springer, 1998.

${ }^{65}$ Willert, C.E. and Gharib, M. "Digital Particle Image Velocimetry." Experiments in Fluids, Vol. 10, 1991, pp. 181-193.

66 Johns Hopkins University, Laboratory for Experimental Fluid Dynamics, http://www.me.jhu.edu/ lefd/particles.htm.

67 Eastman Kodak Co. Kodak Filters for Scientific and Technical Uses. Kodak Publication B-3, 1985. p. 65.

${ }^{68}$ Nelson, R.C. and Pelletier, A. "An Experimental Investigation of Vortex Breakdown on Slender Delta-Wing Planforms.” Notre Dame Contractor Report, August 1994.

69 Klein, M.A., Hubner, J.P. and Komerath, N.M. "Spectral Measurements in Vortex Flow over Swept-winged Configurations and High Angle of Attack." AIAA paper \# 941804, June 1994.

70 Truneva, E.A. "On the Mechanism of Vortex Breakdown Point Stabilization for a Low-Subsonic Flow about a Delta Wing." Bulletin of Institutes of Higher Education, Aviation Tech. (USSR), Vol. 19, No. 2, 1976.

71 Lambourne, N.C. and Bryer, D.W. Reports and Memoranda No. 3282 (also A.R.C. No. 22,775), April 1961.

72 Delery, J.M. "Aspects of Vortex Breakdown." Prog. Aerospace Sci. Vol. 30, pp. 1$59,1994$.

73 Gad-el-Haq, M. and Blackwelder, R. F. " "The Discrete Vortices from a Delta Wing." AIAA Journal. Vol. 23, No. 6, June 1985, pp. 961-962. 
${ }^{74}$ Mangler, K.W. and Smith, J.H.B. "A Theory of the Flow Past a Slender Delta Wing with Leading-Edge Separation." Proc. Roy. Soc. Lond. A, Vol. 251, 1959, pp. 200-217.

75 Brown, C.E. and Michael, W.H. "Effect of Leading-Edge Separation on the Lift of a Delta Wing." Journal of the Aeronautical Sciences, 1954, pp. 690-694.

${ }^{76}$ Fink, P.T. and Taylor, J. "Some Low Speed Experiments with $20^{\circ}$ Delta Wings." Aeronautical Research Council, No. 17,854, 1955.

77 Smith, J.H.B. "Improved Calculations of Leading Edge Separation from Slender, Thin Delta Wings." Proc. Royal Society, London. Vol. A 306, 1968, pp. 67-90.

${ }^{78}$ Huang, Ming-Ke and Chow, Chuen-Yen. "Stability of Leading-Edge Vortex Pair on a Slender Delta Wing." AIAA Journal, Vol. 34, No. 6, June 1996, pp. 1182-1187.

79 Traub, L. "Low-Reynolds-Number Effects on Delta Wing Aerodynamics." J. Aircraft, Vol. 35, No. 4, 1998, pp. 653-656.

${ }^{80}$ Roos, F. W. and Kegelman, J. T. "An Experimental Investigation of Sweep Angle Influence on Delta-Wing Flows." AIAA paper \#A90-22210, 1990.

${ }^{81}$ Hummel, D. "On the Vortex Formation over a Slender Wing at Large Angles of Incidence." AGARD CP-247, paper \#15, 1979, pp.15-1 - 15-17.

${ }^{82}$ Lee, M. and Ho, C.-M. "Vortex Dynamics of Delta Wings" In "Lecture Notes in Engineering", \#46, M. Gad-el-Hak (Ed.); Frontiers in Experimental Fluid Mechanics, pp. 365-427.

${ }^{83}$ White, F.M. Fluid Mechanics. McGraw-Hill, 1988.

${ }^{84}$ Kundu. Fluid Mechanics. Academic Press, 1990.

${ }^{85}$ Lawson, N.J. and Wu, J. "Three-Dimensional Particle Image Velocimetry:

Experimental Error of a Digital Angular Stereoscopic System." Meas. Sci. Technol., Vol. 8, No. 12, December 1997, pp. 1455-1464. 